\title{
Laser ablation-inductively coupled plasma-mass spectrometry: examinations of the origins of polyatomic ions and advances in the sampling of particulates
}

\author{
by
}

Travis Michael Witte

\begin{abstract}
A dissertation submitted to the graduate faculty
in partial fulfillment of the requirements for the degree of

DOCTOR OF PHILOSOPHY
\end{abstract}

Major: Analytical Chemistry

Program of Study Committee:

R. S. Houk, Major Professor

Ning Fang

Aaron D. Sadow

Klaus Schmidt-Rohr

Emily A. Smith

Iowa State University

Ames, Iowa 


\section{Table of Contents}

Chapter 1. Introduction 1

ICP-MS

LA-ICP-MS

Polyatomic Ion Interferences

Laser Sampling Effects 6

Dissertation Organization 9

References $\quad 10$

Chapter 2. Origins of Polyatomic Ions in LA-ICP-MS: An Examination of Metal Oxide Ions \& Effects of Nitrogen and Helium in the Aerosol Gas Flow 14

Abstract $\quad 14$

$\begin{array}{ll}\text { Introduction } & 15\end{array}$

Theory 17

$\begin{array}{ll}\text { Determination of Polyatomic Ion Origin } & 17\end{array}$

Experimental 18

Instrumentation $\quad 18$

$\begin{array}{ll}\text { Materials } & 19\end{array}$

Estimation of Neutral Density $\left(n_{\mathrm{B}}\right) \quad 20$

Mass Bias Corrections $\quad 22$

Results and Discussion $\quad 22$

$\mathrm{MO}^{+}$Ions in Wet vs. Dry Plasmas $\quad 22$

Mixed Gas Plasmas $\quad 24$

$\mathrm{N}_{2}$ and $\mathrm{Ar} \quad 26$

$\mathrm{He}$ and $\mathrm{Ar} \quad 29$

$\mathrm{N}_{2}$, He and $\mathrm{Ar} \quad 31$

Conclusions $\quad 33$

Acknowledgements 34

References

Tables $\quad 40$

Figures $\quad 42$

Chapter 3. Examinations of the Origins of Metal Argide Ions in Laser AblationInductively Coupled Plasma-Mass Spectrometry

Abstract $\quad 55$

Introduction $\quad 55$

Theory $\quad 58$

Determination of Polyatomic Ion Origin $\quad 58$

$\begin{array}{ll}\text { Experimental } & 60\end{array}$

$\begin{array}{ll}\text { Instrumentation } & 60\end{array}$

$\begin{array}{ll}\text { Materials } & 60\end{array}$

Estimation of Neutral Density $\left(n_{\mathrm{Ar}}\right) \quad 61$

Mass Bias Corrections $\quad 61$

Results and Discussion $\quad 62$ 
$\begin{array}{ll}\text { Conclusions } & 68\end{array}$

$\begin{array}{ll}\text { Acknowledgements } & 70\end{array}$

$\begin{array}{ll}\text { References } & 70\end{array}$

Tables $\quad 74$

$\begin{array}{ll}\text { Figures } & 79\end{array}$

Chapter 4. Determination of Uranium Isotope Ratios in Particulate Samples by fs-LA-ICP-MS $\quad \mathbf{8 0}$

Abstract $\quad 80$

$\begin{array}{lr}\text { Introduction } & 80\end{array}$

$\begin{array}{lr}\text { Experimental } & 83\end{array}$

Instrumentation $\quad 83$

Materials $\quad 85$

Results and Discussion $\quad 86$

U Particulate Standard Samples $\quad 86$

$\begin{array}{lr}\text { Environmental Particulates } & 89\end{array}$

$\begin{array}{ll}\text { Conclusions } & 91\end{array}$

Acknowledgements $\quad 92$

References $\quad 92$

Figures $\quad 96$

Tables 106

$\begin{array}{lr}\text { Chapter 5. General Conclusions } & 109\end{array}$

$\begin{array}{ll}\text { Acknowledgements } & 111\end{array}$

Appendix A. Measurement of Gas Kinetic Temperatures for Polyatomic Ions in Inductively Coupled Plasma-Mass Spectrometry: Validation and Refinements $\quad \mathbf{1 1 2}$

$\begin{array}{ll}\text { Abstract } & 113\end{array}$

$\begin{array}{ll}\text { Introduction } & 113\end{array}$

Theory 116

Origin of Polyatomic Ion from Measured $\mathrm{T}_{\text {gas }}$ Values 116

Mass Bias Corrections and Number Densities of Neutral Species 117

$\begin{array}{ll}\text { High Temperature Corrections to the Partition Functions } & 119\end{array}$

$\begin{array}{lr}\text { Correction for Ionization Reactions } & 121\end{array}$

Experimental Section $\quad 122$

Results and Discussion $\quad 125$

Electronic Partition Functions of Diatomic Molecules 125

Effect of High Temperature Rotational-Vibrational Corrections to Partition

$\begin{array}{ll}\text { Functions } & 126\end{array}$

Inclusion of Ionization Energy for Neutral Diatomic Molecules 126

$\begin{array}{lr}\text { Error Analysis } & 128\end{array}$

$\begin{array}{lr}\text { Acknowledgements } & 129\end{array}$

$\begin{array}{lr}\text { References } & 129\end{array}$

$\begin{array}{ll}\text { Tables } & 134\end{array}$ 
Figures

\section{Appendix B. Vanadium Induces Dopaminergic Neurotoxicity Via Protein Kinase C-Delta Dependent Oxidative Signaling Mechanisms: Relevance to Etiopathogenesis of Parkinson's Disease}

140

141

Abstract

Introduction

Methods

Chemicals

Cell culture

Treatment paradigm

Assessment of cell death by Sytox Green assay

3-(4,5-dimethylthiazol-3-yl)-2,5-diphenyl tetrazolium bromide (MTT) assay

Determination of intracellular vanadium concentration

Determination of vanadium-induced $\mathrm{H}_{2} \mathrm{O}_{2}$ production by polarography

Measurement of caspase- 3 and caspase- 9

DNA fragmentation assay

PKC $\delta$ knockdown by siRNA in N27 cells

Protein kinase $\mathrm{C} \delta$ activity

Western blotting

Dopaminergic neuronal viability by ${ }^{3} \mathrm{H}$-Dopamine $\left({ }^{3} \mathrm{H}-\mathrm{DA}\right)$ uptake assay

Statistical analysis

Results

Vanadium exposure induces dose-dependent increase in cytotoxicity

Time-dependent uptake of vanadium in N27 dopaminergic cells

Vanadium induces oxidative stress in N27 mesencephalic neuronal cells

Vanadium exposure promotes mitochondrial cytochorome c release

Vanadium induces a time-dependent activation of caspase- 9 and caspase-3

Caspase- 3 mediates proteolytic cleavage of $\mathrm{PKC} \delta$ in vanadium exposed $\mathrm{N} 27$ cells

Vanadium induces increases in PKC $\delta$ kinase activity in a caspase-dependent

manner

Caspases mediate vanadium-induced apoptotic and cytotoxic cell death in N27 cells

Suppression of PKC $\delta$ by PKC $\delta$ siRNA rescues N27 cells from vanadium-induced apoptic cell death

Vanadium induces neurotoxic responses to primary dopaminergic neurons

Discussion

Acknowledgement

References

Figures and Figure Legends
142

145

145

146

147

148

148

149

150

151

151

152

153

154

155

155

156

156

157

158

159

159

160

161

161

162

163

163

169

170

178 


\section{Chapter 1. Introduction}

\section{ICP-MS}

Inductively coupled plasma-mass spectrometry (ICP-MS) is among the most powerful and sensitive techniques for elemental analysis. The ICP is an atmospheric, high temperature atomization source $(5000-6000 \mathrm{~K})$ capable of vaporizing, atomizing and ionizing a variety of samples [1,2]. Argon is most often chosen as the gas for plasma generation. Plasma ignition is easier in Ar, which requires less energy to heat compared to diatomic gases. The ionization energy of $\operatorname{Ar}(15.76 \mathrm{eV})$, one of the highest found in the periodic table, enables the ICP to effectively ionize the majority of elements [3]. Unlike other available gases, Ar is relatively chemically inert. This inhibits the formation of molecular argide ions with analyte species, an important consideration for atomic spectrometry.

Very early ICPs were used for the growth of crystals of refractory oxides [4]. Adaptation of the ICP for application as an atomic excitation source was begun soon afterward [5]. This work led to the employment of ICPs as emission sources in atomic emission spectrometry (AES), introducing instrumentation still used in many laboratories today. Emission spectra, especially for heavy elements, can be plagued by spectral overlap when multiple elements are present, making interpretation difficult [6]. Incorporation of an ICP source with a mass spectrometer, first achieved by Houk et al. [7], simplified multielement analyses. ICP-MS instruments exhibit both a large dynamic range and low detection limits $(<1$ part per trillion) [8].

Innovations in ICP-MS instrumentation have involved both the integration of different types of MS, including quadrupole [9], magnetic sector [10] and time of flight MS 
[11], and the further development of sample introduction methods. Samples are typically prepared as acidified dilute aqueous solutions and introduced via solution nebulization. Addition of water to the plasma can lead to the formation of undesirable oxide $\left(\mathrm{MO}^{+}\right)$and hydride $\left(\mathrm{MH}^{+}\right)$polyatomic interferences [3]. Desire to reduce these analytically harmful species has spurred the development of desolvation systems for sample introduction [12]. The development of laser ablation (LA) sampling has simplified sample preparation and expanded the scope of samples that can be readily analyzed by ICP-MS.

\section{LA-ICP-MS}

LA-ICP-MS permits direct sampling of solids with little or no sample preparation [13]. A reduction in sample preparation provides a number of advantages for ICP-MS analyses. Complicated and hazardous dissolution procedures involving the use of hydrofluoric acid or heated perchloric acid can be avoided altogether. Contamination from the acids and containers used during digestion can reduce the accuracy of analyses and introduce interfering species to the sample. Sub-boiling distillation of acids and acid vapor washing of containers may be performed to lessen these complications [14]. However, these steps, much like the digestions they precede, are often time-consuming and can greatly extend the duration of the analysis. By removing the necessity of performing these steps, LA-ICP-MS expedites the analytical process.

Another advantage provided by LA-ICP-MS over solution-based analysis is the ability to obtain information on the spatial distribution of elements in the sample. Digestion destroys all structures in the original sample, rendering lateral spatial analysis impossible. Through control of laser spot size and beam profile homogenization, both surface and depth 
profiles can be constructed with LA-ICP-MS. These capabilities have extended the adoption of LA-ICP-MS to a number of fields from geological [15-17] and materials analysis $[18,19]$ to forensic [20,21], environmental [22,23], and even biological applications [24,25].

With the ever increasing use of this technique, a greater emphasis has been placed on addressing the issues that hinder the analytical proficiency of LA-ICP-MS. Some of these issues are the same faced for solution-based ICP-MS, namely the problem of spectral interferences arising from polyatomic species. Others are specific to LA-ICP-MS, chiefly concerns involving the interaction of the laser with the sample surface and subsequent production of solid particulates. Depending upon conditions during the ablation event, a non-representative aerosol may be produced from the sample location ablated by the laser beam [26]. Improvement in these areas will further serve to make LA-ICP-MS an attractive alternative to solution nebulization ICP-MS analysis.

\section{Polyatomic Ion Interferences}

As an ion source for elemental analysis, the ICP is required to fully atomize and ionize the sample for detection in the MS. While this is largely achieved, some polyatomic species remain after ion extraction from the ICP into the MS [3]. Operation of the ICP at temperatures great enough to completely eliminate such polyatomic species would produce an abundance of multiply-charged ions, further complicating spectral analysis [27]. Thus the conditions at which the ICP is commonly used represent an acceptable compromise between the maximization of singly-charged atomic ions and the survival of some polyatomic interferences. Atomic signals measured in the mass spectrum are susceptible to overlap by these polyatomic ions. 
Polyatomic species may arise from incomplete atomization in the ICP itself or reactions in the extraction process between analyte elements and the major background atoms in the ICP $(\mathrm{Ar}, \mathrm{H}, \mathrm{O})[28]$. Whatever the source, the detrimental effect of polyatomic interferences on analytical accuracy has spurred the development of a number of strategies for their eradication.

Attempts to remove or reduce polyatomic ions have taken many forms. Some have relied upon instrumental design. High resolution magnetic sector instruments capable of resolutions $\mathrm{m} / \Delta \mathrm{m}=10,000$ and higher can separate many polyatomic ions from nearby atomic species [10]. This comes at the expense of the ion signal, however, with the measured signal decreasing as much as two orders of magnitude compared to low resolution $(\mathrm{m} / \Delta \mathrm{m}=300)$ results on the same instrument. ICP sources have been incorporated with Fourier-transform ion cyclotron resonance and orbitrap mass spectrometers to achieve even greater resolutions, but cost and sensitivity considerations limit their practical application [29]. Collision cells have been added to ICP-MS instruments to remove polyatomic interferences [30]. An additional gas is added into the MS in a controlled fashion as a means to either change unwanted interfering ions into species that do not overlap with the elements of interest or enable kinetic energy discrimination through collisions with polyatomic ions. If the polyatomic ion is to be removed via a chemical reaction, the choice of gas must be made with regard to the specific polyatomic ions to be removed and may not be effective at dispensing with all interferences in multi-element analyses.

Other procedures for the reduction of polyatomic ions rely upon operational modifications such as cool plasmas $[31,32]$. A cool plasma is produced by operating the ICP at lower RF power and higher Ar gas flow rates. Polyatomic species with high ionization 
energies, like many Ar-based ions, are less abundant at these conditions. While this enables the measurement of elements otherwise overlapped by these polyatomics, many elements with high ionization energies themselves are not ionized. Post-analysis schemes have also been pursued in the form of mathematical corrections $[33,34]$. These must often be tailored to address a single polyatomic interference as even across a class of polyatomic ions, such as oxides, species may affect the spectrum in very different ways.

The described methods for the reduction of polyatomic ions suffer from drawbacks that prohibit their application in many situations. To take advantage of the sensitivity and multi-element detection capabilities of the ICP-MS, remedial strategies that can be applied to complex sample analyses are needed.

If lower basic levels of polyatomic ions can be generally achieved, compromises caused by the measures to reduce them will be less severe. Studies have been performed on the fundamentals behind the formation of polyatomic ions in solution nebulization ICP-MS to help develop a universal reduction in these problematic species $[28,35]$. A similar investigation undertaken for polyatomic interferences arising during LA-ICP-MS is necessary. Samples with complex matrices, a potential source of myriad polyatomic interferences, are commonly analyzed with LA-ICP-MS, with no prior chemical separation to remove the offending species. A unified strategy that maintains the advantages of the LAICP-MS while reducing a majority of possible polyatomic interferences will improve the fitness of the technique for all trace-level analysis. 


\section{Laser Sampling Effects}

In order to be utilized as a distinct analytical technique and not simply a complement to solution nebulization analysis, LA-ICP-MS must be able to achieve the level of accuracy and precision necessary for isotopic and quantitative analyses. The degree to which this is attained is heavily influenced by the ability of the laser to produce an aerosol which correctly represents the bulk composition. Failure in this regard results in an effect known as fractionation, the non-stoichiometric representation of the isotopic or elemental composition of the bulk sample in the mass spectrum [36,37]. Fractionation effects occur both during the ablation event itself, known as laser-induced fractionation, and in the ICP during the conversion of aerosol particle into ions, referred to as ICP-induced fractionation.

Laser-induced fractionation is a consequence of the manner in which energy is deposited into the sample by the laser pulse. While a portion of the laser energy goes to breaking chemical bonds to produce the aerosol, some of the energy simply heats the sample [38]. This enhances the vaporization of volatile elements from the solid. As particles condense within the aerosol, they become enriched with volatile elements relative to the composition of the bulk sample. These volatile elements are then overrepresented in the mass spectrum relative to the actual stoichiometry of the sample.

Beyond contributing to laser-induced fractionation, sample heating can also influence the magnitude of ICP-induced fractionation effects. Melting occurs around the ablation crater as the laser heats the sample. Continued ablation at a single location can eject melt from the crater area. The melt then condenses into large spherical particles which are transported to the ICP [39]. Large-sized particles can also be produced when samples 
weakly absorb energy from the selected laser, which is related to the laser wavelength and sample optical characteristics [36].

Particles larger than $150 \mathrm{~nm}$ are only partially vaporized in the ICP prior to extraction in the mass spectrometer [40]. Volatile elements are vaporized more extensively than refractory elements during this process, increasing their relative abundance in the mass spectrum [37]. When these large particles lay off-axis to the extraction orifice, the nonstoichiometric ion cloud surrounding the particle is not sampled uniformly by the mass spectrometer and the accuracy of the analysis is diminished by ICP-induced fractionation. Large particles can also prove detrimental to analytical precision should they be transported on-axis with the extraction orifice [41]. In these instances partial vaporization immediately before extraction can produce large spikes on the signal transient as the entire ion cloud is sampled together by the mass spectrometer, reducing the precision of the measurement.

Several modifications have been made to laser ablation procedures to diminish fractionation from all sources. Smaller average particle sizes have been obtained by employing UV lasers [36]. Energy transfer to the sample surface is enhanced at shorter wavelengths, improving the breakdown of the ablated material into smaller particles. Helium is frequently substituted for argon as the carrier gas through the ablation cell. The increased thermal conductivity of He more efficiently disperses heat away from the ablation location [42]. This curtails melting in and around the ablation crater, inhibiting the formation and transport of large spherical melt particles [43]. ICP-induced fractionation effects are additionally lessened due to this property. Helium as the carrier gas in the ICP better conducts energy to the central channel and improves vaporization of the sample aerosol. 
Pulse duration has been identified as another key aspect in dictating the constitution of the sample aerosol [37-39,44,45]. Nanosecond lasers have long been the most economical systems available for LA-ICP-MS, but recent advances have increased the practicality of fs lasers for ICP-MS laboratories. The shorter interval in which the fs laser pulse interacts with the solid sample presents numerous advantages over ns lasers. Typically on the order of $\sim 100 \mathrm{fs}$, the time scale of the fs pulse is shorter than that over which heating can occur in the solid. Material is removed from the surface before any of the negative consequences from heating are possible. The large spherical particles observed during ablation with ns lasers are not present when fs lasers are used. Instead, many small, irregular particles ( 100-200 nm diameter) are created [45]. These agglomerates come apart some time during transport and introduction into the ICP, breaking into small particles manageably vaporized and atomized.

Such a particle formation process is observed when using fs lasers with many different sample matrices as opposed to ablation analyses with ns lasers, where fractionation effects can be both greater and more variable with sample type $[39,46]$. This aspect of fsLA-ICP-MS may help to overcome an important challenge since the inception of LA-ICPMS, the need for matrix-matched standards for accurate calibrations [47]. With matrixindependent particle formation, external standards for quantification and mass bias corrections need not be exactly the same type of matrix as the sample to be characterized. Ablation with a fs laser improves the accuracy of quantitative and isotopic analysis for a broad scope of samples [48].

Among the sample types benefiting from this improvement are particulate matter samples. LA-ICP-MS is most commonly applied to the analysis of bulk solid samples, for which matrix-matched standards can be utilized if available [13]. Matching standards to 
particulates is more difficult. Particulates may be collected on media such as filters or swipes from the atmosphere or surfaces, resulting in a variety of materials constituting the total sample matter [22]. The morphology and composition of the particulate matter is often unknown and indeed may not be the same for all particulates in a single collected sample. Adoption of fs-LA facilitates the analysis of these samples by permitting use of unmatched standards with reasonable quantitative results.

The diminished surface heating effects from fs lasers are also advantageous for particulate samples. Distinct particulates or particulate ensembles are selected for ablation from the substrate. Alteration of the sample surface surrounding the ablation crater may liberate particulates not directly selected or affect their removal in subsequent ablations. Either phenomenon can introduce undesirably large particulates into the ICP. Affixing the samples to the collection medium with an immobilizer mitigates this effect somewhat, but the immobilizer is still prone to heating when a ns laser is used. Ablation of particulate samples incorporating both an immobilizer and fs laser may provide improved precision and maintain information on the spatial distribution of elements. Validation of this technique may enable a number of applications from environmental monitoring of hazardous materials to quality assurance in production of nanoparticles.

\section{Dissertation Organization}

This dissertation is organized into five chapters. Chapter 1 provides a general introduction to ICP-MS and LA sampling, with an examination of analytical challenges in the employment of this technique. Chapter 2 presents a manuscript prepared for submission to Spectrochimica Acta Part B. It discusses the origin of metal oxide ions $\left(\mathrm{MO}^{+}\right)$in LA-ICP- 
MS, as well as the effect of introducing helium and nitrogen to the aerosol gas flow on the formation of these polyatomic interferences. Chapter 3 features another manuscript prepared for submission to Spectrochimica Acta Part B. It extends the study of polyatomic ions in LA-ICP-MS to metal argide $\left(\mathrm{MAr}^{+}\right)$species, an additional source of possible significant interferences in the spectrum. Chapter 4 describes the application of fs-LA-ICP-MS to the determination of uranium isotope ratios in particulate samples. Chapter 5 summarizes conclusions from the above studies and considers possible directions for the continued investigation of these and related issues in LA-ICP-MS. Appendix A is a paper published in Spectrochimica Acta Part B for which I contributed supplemental experimental data and analysis. Appendix B is a paper published in Toxicology and Applied Pharmacology for which I contributed an analysis of the trace metal content of treated cell cultures.

\section{References}

[1] R.S. Houk, J.J. Thompson, Inductively coupled plasma mass spectrometry, Mass Spec. Rev. 7 (1988) 425-461.

[2] K.E. Jarvis, A.L. Gray, R.S. Houk, Handbook of Inductively Coupled Plasma Mass Spectrometry (Chapman and Hall, New York, 1992).

[3] H. Niu, R.S. Houk, Fundamental aspects of ion extraction in inductively coupled plasma mass spectrometry, Spectrochim. Acta Part B 51 (1996) 779-815.

[4] T.B. Reed, Induction-Coupled Plasma Torch, J. Appl. Phys. 32 (1961) 821-824.

[5] R.H. Wendt, V.A. Fassel, Induction-Coupled Plasma Spectrometric Excitation Source, Anal. Chem. 37 (1965) 920-922.

[6] S. Gu, H. Ying, Z. Zhang, Z. Zhuang, P. Yang, X. Wang, B. Huang, B. Li, High resolution spectra of selected rare earth elements and spectral interferences studied by inductively coupled plasma-atomic emission spectroscopy (ICP-AES), Spectrochim. Acta Part B 52 (1997) 1567-1574.

[7] R.S. Houk, V.A. Fassel, G.D. Flesch, H.J. Svec, A.L. Gray, C.E. Taylor, Inductively Coupled Argon Plasma as an Ion Source for Mass Spectrometric Determination of Trace Elements, Anal. Chem. 52 (1980) 2283-2289. 
[8] K.J. Stetzenbach, M. Amano, D.K. Kreamer, V.F. Hodge, Testing the Limits of ICPMS: Determination of Trace Elements in Ground Water at the Part-Per-Trillion Level, Ground Water 32 (1994) 976-985.

[9] P.E. Miller, M.B. Denton, The quarupole mass filter: basic operating concepts, J. Chem. Ed. 63 (1986) 617-622.

[10] N. Bradshaw, E.F.H. Hall, N.E. Sanderson, Inducitvely coupled plasma as an ion source for high-resolution mass spectrometry, J. Anal. At. Spectrom. 4 (1989) 801803.

[11] D.P. Myers, G.M. Hieftje, Preliminary Design Considerations and Characteristics of an Inductively Coupled Plasma-Time of Flight Mass Spectrometer, Microchem. J. 48 (1993) 259-277.

[12] M.G. Minnich, R.S. Houk, Comparison of cryogenic and membrance desolvation for attenuation of oxide, hydride and hydroxide ions and ions containing chlorine in inductively coupled plasma mass spectrometry, J. Anal. Atom. Spectrom. 13 (1998) 167-174.

[13] B. Hattendorf, C. Latkoczy, D. Güenther, Laser ablation-ICPMS, Anal. Chem. 75 (2003) 341A-347A.

[14] H. Yuan, S. Hu, J. Tong, L. Zhao, S. Lin, S. Gao, Preparation of ultra-pure water and acids and investigation of background of an ICP-MS laboratory, Talanta 52 (2000) 971-981.

[15] L.A. Solari, A. Gomez-Tuena, J.P. Bernal, O. Perez-Arvizu, M. Tanner, U-Pb geochronology with an integrated LA-ICP-MS microanalytical workstation: achievements in precision and accuracy, Geostand. Geoanal. Res. 33 (2010) 309-317.

[16] R. Arevalo, Jr., W.F. McDonough, P.M. Piccoli, In situ determination of first-row transition metal, Ga and Ge abundances in geological materials via medium resolution LA-ICP-MS, Geostand. Geoanal. Res. 35 (2011) 253-273.

[17] C.M. Fisher, C.R.M. McFarlane, J.M. Hanchar, M.D. Schmitz, P.J. Sylvester, R. Lam, H.P. Longerich, Sm-Nd isotope systematics by laser ablation-multicollectorinductively coupled plasma mass spectrometry: Methods and potential natural and synthetic reference materials, Chem. Geol. 284 (2011) 1-20.

[18] A. Michalska, M. Wojciechowski, E. Bulska, K. Maksymiuk, Experimental study on stability of different solid contact arrangements of ion-selective electrodes, Talanta 82 (2010) 151-157.

[19] J.S. Lee, H.B. Lim, Laser ablation ICP-MS to determine $\mathrm{Cu}$ on a Si wafer prepared by ion sputtering, J. Anal. Atom. Spectrom. 26 (2011) 1534-1538.

[20] M.I. Szynkowska, K. Czerski, T. Paryjczak, A. Parczewski, Ablative analysis of black and colored toners using LA-ICP-TOF-MS for the forensic discrimination of photocopy and printer toners, Surf. Interface Anal. 42 (2010) 429-437.

[21] P. Weis, M. Duecking, P. Watzke, S. Menges, S. Becker, Establishing a match criterion in forensic comparison analysis of float glass using laser ablation inductively coupled plasma mass spectrometry, J. Anal. Atom. Spectrom. 26 (2011) 1273-1284.

[22] Y.-K. Hsieh, L.-K. Chen, H.-F. Hsieh, C.-H. Huang, C.-F. Wang, Elemental analysis of airborne particulate matter using an electrical low-pressure impactor and laser ablation/inductively coupled plasma mass spectrometry, J. Anal. Atom. Spectrom. 26 (2011) 1502-1508. 
[23] A. Ugarte, N. Unceta, C. Pecheyran, M.A. Goicolea, R.J. Barrio, Development of matrix-matching hydroxyapatite calibration standards for quantitative multi-elements LA-ICP-MS analysis: application to the dorsal spine of fish, J. Anal. Atom. Spectrom. 26 (2011) 1421-1427.

[24] I. Konz, B. Fernandez, M.L. Fernandez, R. Pereiro, A. Sanz-Medel, Absolute Quantification of Human Serum Transferrin by Species-Specific Isotope Dilution Laser Ablation ICP-MS, Anal. Chem. 83 (2011) 5353-5360.

[25] Z. Qin, J.A. Caruso, B. Lai, A. Matusch, J.S. Becker, Trace Metal imaging with high spatial resolution: Applications in biomedicine, Metallomics 3 (2011) 28-37.

[26] R. Hergenröder, A model of non-congruent laser ablation as a source of fractionation effects in LA-ICP-MS, J. Anal. At. Spectrom. 21 (2006) 505-516.

[27] K.E. Jarvis, A.L. Gray, E. McCurdy, Avoidance of spectral interference on europium in inductively coupled plasma mass spectrometry by sensitive measurement of the doubly charged ion, J. Anal. At. Spectrom. 4 (1989) 743-747.

[28] R.S. Houk, N. Praphairaksit, Dissociation of polyatomic ions in inductively coupled plasma, Spectrochim. Acta Part B 56 (2001) 1069-1096.

[29] K.E. Milgram, F.M. White, K.L. Goodner, C.H. Watson, D.W. Koppenaal, C.J. Barinaga, B.H. Smith, J.D. Winefordner, A.G. Marshall, R.S. Houk, J.R. Eyler, HighResolution Inductively Coupled Plasma Fourier Transform Ion Cyclotron Resonance Mass Spectrometry, Anal. Chem. 69 (1997) 3714-3721.

[30] S.D. Tanner, V.I. Baranov, D.R. Bandura, Review: Reaction cells and collision cells for ICP-MS: a tutorial review, Spectrochim. Acta Part B 57 (2002) 1361-1452.

[31] S.D. Tanner, Characterization of Ionization and Matrix Suppression in Inductively Coupled 'Cold' Plasma Mass Spectrometry, J. Anal. At. Spectrom. 10 (1995) 905-921.

[32] K.Y. Patterson, C. Veillon, A.D. Hill, P.B. Moser-Veillon, T.C. O'Haver, Measurement of calcium stable isotope tracers using cool plasma ICP-MS, J. Anal. At. Spectrom. 14 (1999) 1673-1677.

[33] J. Goossens, L. Moens, R. Dams, A mathematical correction method for spectral interferences on selenium in inductively coupled plasma mass spectrometry, Talanta 41 (1994) 187-193.

[34] F. Laborda, M. Górriz, E. Bolea, J.R. Castillo, Mathematical correction for polyatomic interferences in the speciation of chromium by liquid chromatographyinductively coupled plasma quadrupole mass spectrometry, Spectrochim. Acta Part B 61 (2006) 433-437.

[35] J.W. Ferguson, R.S. Houk, High resolution studies of the origins of polyatomic ions in inductively coupled plasma-mass spectrometry, Part I. Identification methods and effects of neutral gas density assumptions, extraction voltage, and cone material, Spectrochim. Acta Part B 61 (2006) 905-915.

[36] M. Guillong, D. Günther, Effect of particle size distribution on ICP-induced elemental fractionation in laser ablation-inductively coupled plasma-mass spectrometry, J. Anal. At. Spectrom. 17 (2002) 831-837.

[37] N.J. Saetveit, S.J. Bajic, D.P. Baldwin, R.S. Houk, Influence of particle size on fractionation with nanosecond and femtosecond laser ablation in brass by online differential mobility analysis and inductively coupled plasma mass spectrometry, J. Anal. At. Spectrom. 23 (2008) 54-61. 
[38] J. González, S.H. Dundas, C. Liu, X. Mao, R.E. Russo, UV-femtosecond and nanosecond laser ablation-ICP-MS: internal and external repeatability, J. Anal. Atom. Spectrom. 21 (2006) 778-784.

[39] J. Koch, A. von Bohlen, R. Hergenröder, K. Niemax, Particle size distributions and compositions of aerosols produced by near-IR femto- and nanosecond laser ablation of brass, J. Anal. At. Spectrom. 19 (2004) 267-272.

[40] H.-R. Kuhn, M. Guillong, D. Güenther, Size-related vaporisation and ionisation of laser-induced glass particles in the inductively coupled plasma, Anal. Bioanal. Chem. 378 (2004) 1069-1074.

[41] D.C. Perdian, S.J. Bajic, D.P. Baldwin, R.S. Houk, Time-resolved studies of particle effects in laser ablation inductively coupled plasma-mass spectrometry. Part 1. Investigation of nanosecond and femtosecond pulse width lasers and devices for particle size selection, J. Anal. At. Spectrom. 23 (2008) 325-335.

[42] A.B. Murphy, Transport Coefficients of Helium and Argon-Helium Plasmas, IEEE T. Plasma Sci. 25 (1997) 809-814.

[43] Z. Wang, B. Hattendorf, D. Günther, Analyte Response in Laser Ablation Inductively Coupled Plasma Mass Spectrometry, J. Am. Soc. Mass Spectrom. 17 (2006) 641-651.

[44] R.E. Russo, X. Mao, J.J. González, S.S. Mao, Femtosecond laser ablation ICP-MS, J. Anal. At. Spectrom. 17 (2002) 1072-1075.

[45] C. Liu, X. Mao, S.S. Mao, X. Zeng, R. Greif, R.E. Russo, Nanosecond and Femtosecond Laser Ablation of Brass: Particulate and ICP-MS Measurements, Anal. Chem. 76 (2004) 379-383.

[46] M. Gaboardi, M. Humayun, Elemental fractionation during LA-ICP-MS analysis of silicate glasses: implications for matrix-independent standardization, J. Anal. At. Spectrom. 24 (2009) 1188-1197.

[47] Q. Bian, C.C. Garcia, J. Koch, K. Niemax, Non-matrix matched calibration of major and minor concentrations of $\mathrm{Zn}$ and $\mathrm{Cu}$ in brass, aluminum and silicate glass using NIR femtosecond laser ablation inductively coupled plasma mass spectrometry, J. Anal. At. Spectrom. 21 (2006) 187-191.

[48] B. Fernandez, C. Pecheyran, O.F.X. Donard, F. Claverie, Direct analysis of solid samples by fs-LA-ICP-MS, TrAC-Trend. Anal. Chem. 26 (2007) 951-966. 


\title{
Chapter 2. Origins of Polyatomic Ions in LA-ICP-MS:
}

\section{An Examination of Metal Oxide Ions \& Effects of Nitrogen and Helium in the Aerosol Gas Flow}

Travis M. Witte and R.S. Houk

\begin{abstract}
Differences in the origins of polyatomic ions in wet plasma conditions, as exist in solution nebulization inductively coupled plasma-mass spectrometry (ICP-MS), versus dry plasma conditions, found in laser ablation (LA)-ICP-MS, are investigated. Silicate and metallic samples are ablated and gas kinetic temperature $\left(\mathrm{T}_{\text {gas }}\right)$ is measured to establish the origins of metal oxide $\left(\mathrm{MO}^{+}\right)$ions. $\mathrm{MO}^{+}$ion abundances observed from both the ablation of silicate samples and metallic samples are found to correspond to formation of the polyatomic ion in the ICP. The same strategy is applied to oxide ratios measured when additional gases $\left(\mathrm{N}_{2}\right.$ and $\mathrm{He}$ ) are introduced into the aerosol gas flow. $\mathrm{N}_{2}$ is found to decrease the $\mathrm{MO}^{+} / \mathrm{M}^{+}$ signal ratio by maximizing atomic sensitivity at lower total gas loads. The addition of $\mathrm{He}$ reduces polyatomic ions throughout the plasma. Use of both $\mathrm{N}_{2}$ and He produces the lowest $\mathrm{MO}^{+} / \mathrm{M}^{+}$signal ratio and highest $\mathrm{T}_{\text {gas }}$ values at the position sampled in the ICP. By gaining a better understanding of the origin of polyatomic ions in LA-ICP-MS and their behavior in mixed gas plasmas, perhaps strategies can be developed to minimize polyatomic interferences in the mass spectrum.
\end{abstract}




\section{Introduction}

The popularity of ICP-MS for elemental and isotopic analysis rests upon its ability to efficiently atomize and ionize the sample [1-4]. Even though the ICP is the most effective atomization source available, it is nonetheless unable to completely eliminate polyatomic ions in the spectrum [5-9]. These polyatomic species cause spectral interferences, producing peaks that overlap with atomic ions present at the same nominal mass-to-charge $(\mathrm{m} / \mathrm{z})$ value. This degrades detection limits and reduces the accuracy of analyses.

Prior studies of polyatomic ions in ICP-MS have resulted in a number of strategies to reduce their impact. These strategies include cool plasmas [10,11], collision and reaction cells [12-16], mathematical corrections [17-20], and high resolution mass analyzers [21,22]. While these methods are effective in dealing with polyatomic interferences, each also has drawbacks that may render them less than ideal for particular applications. Rather than focus on situational remediation techniques, study of the origin of these species in the ICP-MS can provide a fundamental understanding of the formation of polyatomic interferences. Perhaps, more encompassing and effective techniques or instrumental developments to eliminate these interferences from the spectrum may be possible.

The work presented here applies this fundamental approach to polyatomic ions produced in LA-ICP-MS. Earlier investigations conducted both in our group and elsewhere have chiefly described polyatomic interferences produced during traditional solution nebulization analyses, where the "wet" plasma conditions have been well-characterized [5,79,21,23-27]. Conversely, polyatomic ions from the "dry" plasma conditions present in LAICP-MS have not been investigated extensively [28-30]. 
A number of considerations may affect the abundance of polyatomic ions produced in a dry plasma as compared to a wet plasma. An advantage of LA-ICP-MS is the ability to perform analyses with little to no sample preparation [31]. Because of this, however, matrix elements that might be removed during sample preparation in solution-based analyses are introduced into the ICP during the ablation event. This provides a ready source of species for the formation of undesirable polyatomic interferences. The degree of polyatomic ions in the spectrum may also be affected by the nature of the ablated aerosol. Sample aerosol characteristics depend upon the interaction of the laser with the sample substrate, condensation of ablated particles, and transport effects as the aerosol travels from the ablation cell to the ICP-MS [32]. Additional gases other than argon are often employed in the plasma in LA-ICP-MS. Along with the absence of water loading, these gases result in a somewhat different plasma environment in which polyatomics may be formed [33].

The purpose of this work is to examine the origin of polyatomic ions in LA-ICP-MS analyses, both in comparison to solution nebulization analyses and for a variety of aerosol gas compositions often used in ablation analyses. Of the common polyatomic species, $\mathrm{MO}^{+}$ ions are among the most persistent and troublesome interferences found in an ICP-MS spectrum. They are the most abundant polyatomic ions seen during LA. This study will focus on these $\mathrm{MO}^{+}$ions to more fully understand these interferences in LA-ICP-MS. 


\section{Theory}

\section{Determination of Polyatomic Ion Origin}

A general method has been developed to describe the origin of polyatomic ions in ICP-MS $[23,27,34,35]$. This method is based upon the proposed dissociation reaction for the generic ion $\mathrm{AB}^{+}$.

$$
\mathrm{AB}^{+} \rightleftharpoons \mathrm{A}^{+}+\mathrm{B} \quad \Delta \mathrm{H}=\mathrm{D}_{0}\left(\mathrm{AB}^{+}\right)
$$

Eq. 1

A dissociation expression can then be constructed using experimentally collected data.

$$
K_{d}=\left(\frac{n_{A^{+}}}{n_{A B^{+}}}\right) n_{B}
$$

The measured signal ratio between the ions $\mathrm{A}^{+}$and $\mathrm{AB}^{+}$is taken to be proportional to the ratio between number densities $(n)$ for $\mathrm{A}^{+}$and $\mathrm{AB}^{+}$, which eliminates the need to determine absolute ion densities. The neutral species number density $\left(n_{\mathrm{B}}\right)$ is estimated from known plasma conditions. The equilibrium expression can also be written in terms of the appropriate partition functions (z) of each species in the reaction. Table 1 lists the spectroscopic data $(\omega=$ vibrational constant, $B=$ rotational constant, $g=$ statistical weight of the ground state) for $\mathrm{AB}^{+}$(i.e., $\mathrm{MO}^{+}$ions) used in these calculations in the present work $[35,36]$.

$$
K_{d}=\frac{Z_{A^{+}} Z_{B}}{Z_{A B^{+}}} e^{-D_{0} / k T}
$$

Both the neutral number density and the partition functions are dependent upon the gas kinetic temperature $\left(\mathrm{T}_{\mathrm{gas}}\right)$ and must be calculated at each value across a range of $\mathrm{T}_{\text {gas }}$ values. 
The measured dissociation constant $\left(\mathrm{K}_{\mathrm{d}}\right)$ from Eq. 2 and theoretical dissociation constant from Eq. 3 are compared at each $\mathrm{T}_{\text {gas }}$ across this range. The value at which they most closely agree is considered to be the $\mathrm{T}_{\text {gas }}$ indicative of the origin of the polyatomic ion.

Based on these $T_{\text {gas }}$ values, there are three general assignments that can be made for the origin of the polyatomic ion. (1) If the measured $\mathrm{T}_{\text {gas }}$ falls in the range 5000 to $6000 \mathrm{~K}$, the polyatomic ion is present in the spectrum at levels consistent with plasma conditions and is not being affected by post-plasma effects in the interface. (2) If the assigned $\mathrm{T}_{\text {gas }}$ is greater than $6000 \mathrm{~K}$, the polyatomic ion is being removed by some process during ion extraction and thus is less abundant in the spectrum than expected based on plasma conditions alone. (3) If the measured $\mathrm{T}_{\text {gas }}$ value is below $5000 \mathrm{~K}$, the polyatomic ion is more abundant in the spectrum than expected from plasma conditions, so excess polyatomic ion is being formed by some process during ion extraction $[23,25]$.

\section{Experimental}

\section{Instrumentation}

All experiments were performed on a Thermo Finnigan (Bremen, Germany) ELEMENT 1 ICP-MS. The ELEMENT 1 is a high resolution magnetic sector instrument, capable of operation at three defined resolutions. Low resolution $(\mathrm{m} / \Delta \mathrm{m}=300)$ was used when possible to maximize sensitivity. Medium resolution $(\mathrm{m} / \Delta \mathrm{m}=4000)$ was employed when polyatomic ions could not be resolved from nearby atomic ions. An ICP torch with a grounded shield was used throughout this study to minimize secondary discharge effects from the plasma and the kinetic energy spread of ions. Gas flows and ion optics were tuned to optimize signal sensitivity and stability by nebulization of standard solutions initially. The 
instrument was then set up for laser ablation and final adjustments of the aerosol gas flow settings were made.

A nanosecond laser system, the LSX-500 (CETAC Technologies, Inc., Omaha, NE), was used for the majority of the ablation experiments. The LSX-500 is a Nd:YAG laser operated at $266 \mathrm{~nm}$ with a pulse width of $5 \mathrm{~ns}$ and energy of $9 \mathrm{~mJ}$. Laser energy, repetition rate and spot size can be adjusted through software controls. Typical operating conditions for the ICP-MS device and laser are described in Table 2.

During argon-only experiments, all gas flows were supplied through the internal mass flow controllers of the ELEMENT. For ablation in helium, an external mass flow controller (Matheson Tri-Gas, Inc., Basking Ridge, NJ) was used to introduce the gas flow into the ablation cell. External mass flow controllers were also used to regulate argon make-up gas and nitrogen gas flows. All flow rates cited include correction factors for the different gases relative to Ar. The argon make-up gas was combined with the sample aerosol post-ablation cell via a Y-connector. When nitrogen was required it was blended with the sample aerosol through an additional Y-connector downstream of the added argon make-up gas.

\section{Materials}

A custom silicate disk containing several rare earth elements (REEs) was created by Dr. Scott Schlorholtz (Department of Civil, Construction, and Environmental Engineering, Iowa State University) for this study to allow for a wider survey of metal oxide species. Reductions in sensitivity at the higher resolutions necessary to resolve between $\mathrm{M}^{+}$and $\mathrm{MO}^{+}$ ions (e.g., ${ }^{140} \mathrm{CeO}^{+}$vs. ${ }^{156} \mathrm{Gd}^{+}, \mathrm{m} / \Delta \mathrm{m} \sim 7000$ ) resulted in poor signals for polyatomic species and rendered common laser ablation standards, such as the NIST 61X series, not optimum for 
this study. The disk was prepared by silicate glass fusion by adding rare earth oxide powders to a silicon dioxide base. A silicate-based sample was chosen as a suitable proxy for mineral samples due to the similar behavior exhibited by both sample types during laser ablation. Oxide powders were added as necessary to achieve a nominal elemental concentration of 2000 parts per million for each rare earth. The specific elements selected (Y, Cs, Ce, Tb, Tm and Ta) were chosen to avoid spectral overlap and enable analysis in low resolution to maximize analytical sensitivity, especially for $\mathrm{MO}^{+}$ions. Elements were also selected to cover a mass range adequate for the construction of an instrument response curve for mass bias corrections. This disk was used for examination of $\mathrm{YO}^{+}$formation from ablation of silicate samples. A sample of Y metal (Materials Preparation Center, Ames Laboratory, U.S. Department of Energy, Ames, IA) was used to study $\mathrm{YO}^{+}$formation from ablation of a metallic matrix.

\section{Estimation of Neutral Density $\left(n_{B}\right)$}

In order to calculate the measured dissociation constant, the neutral density must be estimated based upon known plasma conditions. For the study of $\mathrm{MO}^{+}$ions, this involves the determination of the density of neutral oxygen $\left(n_{\mathrm{O}}\right)$. This has been achieved for solution nebulization in earlier work through the following equation [23].

$$
n_{O}=\frac{N_{A} L T_{\text {room }}}{F M_{\mathrm{H}_{2} \mathrm{O}} T_{\text {gas }} 1000}
$$

In Eq. $4 \mathrm{~N}_{\mathrm{A}}$ is Avogadro's number, $\mathrm{L}$ is the measured solvent load $\left(\mathrm{g} \mathrm{min}^{-1}\right), \mathrm{T}_{\text {room }}$ is room temperature $(\mathrm{K}), \mathrm{F}$ is the aerosol gas flow rate $\left(\mathrm{L} \mathrm{min}^{-1}\right)$ and $\mathrm{M}_{\mathrm{H}_{2} \mathrm{O}}$ is the molar mass of water $\left(\mathrm{g} \mathrm{mol}^{-1}\right)$. The measured solvent load is typically determined by recording the amount of 
water sampled into the plasma over a defined time period. It is assumed that water is the chief source of oxygen. This strategy is effective for wet plasma conditions where solution nebulization is used for sample introduction. It is less directly applicable to the dry plasma in LA-ICP-MS, where no solvent is involved.

Without solvent to provide the bulk of the oxygen, it is expected that the oxygen found in the plasma under dry conditions would be entrained from the atmosphere or present as an impurity in the argon. Some oxygen may be introduced by ablation of samples, especially those with silicate matrices, but this contribution is observed to be small. This loading is difficult to quantify directly and complicates the calculation of the density of oxygen in the ICP.

A strategy was developed to address this issue by deriving the oxygen number density for a dry plasma from the measured solvent load of a wet plasma. This requires measuring the solvent load during solution nebulization initially. During these measurements, the signal of ${ }^{18} \mathrm{O}^{+}$is collected (Fig. 1a). Operation of the instrument in medium resolution ensures that the ${ }^{18} \mathrm{O}^{+}(\mathrm{m} / \mathrm{z}=17.99916)$ peak is fully resolved from the nearby $\mathrm{H}_{2}{ }^{16} \mathrm{O}^{+}$peak $(\mathrm{m} / \mathrm{z}=$ 18.01057) $\left(\mathrm{m} / \Delta \mathrm{m}=1640\right.$, data for $\mathrm{H}_{2}{ }^{16} \mathrm{O}^{+}$peak not shown $)$. The instrument is then switched to laser ablation sampling and the signal of ${ }^{18} \mathrm{O}^{+}$is again measured (Fig. 1b).

During either solution nebulization or laser ablation, the measured $\mathrm{O}$ density is assumed to be proportional to the ${ }^{18} \mathrm{O}^{+}$signal. Using $n_{\mathrm{O}}$ from the measured solvent load (Eq. 4), a relationship can be constructed to calculate $\mathrm{O}$ density in the ICP. This same proportionality value is used to calculate the neutral density of oxygen across the $T_{\text {gas }}$ range of interest during LA. 


\section{Mass Bias Corrections}

Ion ratios, such as those investigated in this work, are prone to mass bias effects inherent in ICP-MS analyses. Mass bias is corrected for by creating an instrument response curve, as described by Ingle et al. and Ferguson et al. [25,37]. Atomic ion signals, corrected for isotopic abundance and ionization efficiency, are plotted against their respective nominal atomic masses to generate the response curve. These response curves require elements in known concentrations that span the mass range of interest. In solution nebulization ICP-MS, a custom multielement solution can be prepared for this purpose.

For LA-ICP-MS matrix-matched samples containing the appropriate elements are necessary. Ideally the sample being ablated would itself include the desired elements. This was achieved by design with the custom REE disks. The stainless steel CRM also presented elements across a mass range appropriate for this purpose. A suite of elements was measured during each ablation analysis in addition to the atomic and polyatomic ions of interest.

\section{Results and Discussion}

\section{$\mathrm{MO}^{+}$Ions in Wet vs. Dry Plasmas}

Earlier studies have established the nature of polyatomic ion interferences in wet or desolvated plasmas $[5,7,9,21,23-25,38,39]$. Here the ready availability of $\mathrm{O}$ and $\mathrm{H}$ from the solvent leads to the formation of oxide ions, hydride ions and argide ions from the argon supporting the plasma itself. Absent solvent, dry plasmas feature less available $\mathrm{O}$ and $\mathrm{H}$ for the formation of interferences, as evidenced in Fig. 1. Based on our method to determine solvent load, at a given temperature $n_{\mathrm{O}}$ was approximately 2 orders of magnitude lower 
during LA compared to wet conditions, even though the matrix being ablated was $\mathrm{SiO}_{2}$. This would correspond to a nearly 200-fold drop in $n_{\mathrm{H}}$.

Given the decrease in $\mathrm{O}$ and $\mathrm{H}$ abundances in the plasma under dry conditions, polyatomic species incorporating those ions are less abundant than in wet plasmas. Table 3 compares the ratios of $\mathrm{YO}^{+} / \mathrm{Y}^{+}$during solution and laser ablation analyses. The observed $\mathrm{YO}^{+} / \mathrm{Y}^{+}$ratio decreases by nearly the same amount as $n_{\mathrm{O}}$ when solution nebulization and laser ablation are compared. The reduced abundance of oxygen atoms in the dry plasma directly correlates into a reduction in the impact of oxide interferences on the mass spectrum. This holds true for both metallic and silicate matrices, indicating that any contribution of oxygen from the sample to the formation of $\mathrm{MO}^{+}$ions is insignificant.

$\mathrm{YO}^{+}\left(\mathrm{D}_{0}=7.24 \mathrm{eV}\right)$ was observed in both solution and laser ablation analyses at abundances corresponding to $\mathrm{T}_{\text {gas }} \sim 5200 \mathrm{~K}$, values indicative of formation in the plasma alone. The solution result agrees well with the range of $\mathrm{T}_{\text {gas }}$ found in previous studies of dissolved REEs in ICP-MS [23]. Close agreement is also found between the $\mathrm{T}_{\text {gas }}$ values determined from ablation of Y glass and metallic samples (Fig. 2) within a substantial range of Ar gas flow rates $(0.925 \mathrm{~L} / \mathrm{min}$ to $1.25 \mathrm{~L} / \mathrm{min})$. LA of silicate samples produces particles that are mainly of a size distribution that is readily vaporized, atomized and ionized in the ICP when ablated, although some large, spherical particles are also observed [29]. With more particles of a common size regime, atomization occurs in a discrete zone in the plasma much like for solution aerosols.

Similar results are seen from the ablation of the Y metal sample, despite the increased possibility of large particles in the aerosol. Heating and subsequent melting of metallic samples by the laser pulse can cause a greater incidence of large particles during the ablation 
$[32,40]$. These particles may be expected to contribute $\mathrm{MO}^{+}$ions to the spectrum if not fully atomized. The low oxygen content of the metallic sample, however, helps prevent this undesirable effect. As observed for both sample types, the shared origin of $\mathrm{YO}^{+}$in the plasma is itself an expression of the common experience of ablated particles and solution aerosols in the ICP. This link enables use of the $\mathrm{YO}^{+} / \mathrm{Y}^{+}$ratio, as well as the ratios of similar species like $\mathrm{CeO}^{+} / \mathrm{Ce}^{+}$, as probes to determine $\mathrm{T}_{\text {gas }}$ at the sampled position in the ICP.

During LA, absolute ion signals of $10^{3}$ and $10^{6}$ counts $\mathrm{s}^{-1}$ were observed for $\mathrm{YO}^{+}$ from the silicate and metallic samples, respectively. The observed ratios used peak integrated signals of $10^{8}$ and $10^{10}$ counts s${ }^{-1}$ for both atomic ions, however, which were only reached due to the high concentrations of each element (Glass: $\mathrm{Y}=9.45 \%$, Metal: $\mathrm{Y}>$ 99.9\%). Thus, these $\mathrm{MO}^{+}$ions are much less abundant during LA than from nebulized solutions, as expected. Similar oxide percentages in this range $(0.001 \%-0.01 \%)$ would result in much lower absolute polyatomic ion signals if formed from trace elements, greatly reducing their impact on the spectrum. Nonetheless, such $\mathrm{MO}^{+}$ions from minor or trace constituents can still be problematic in LA-ICP-MS. The convenience of little to no sample preparation comes with the caveat that all matrix elements will be sampled alongside the analyte elements of interest. Strongly bound polyatomic ions, like $\mathrm{MO}^{+}$, should therefore be considered as possible interferences to be accounted for when developing a method based on laser ablation.

\section{Mixed Gas Plasmas}

Gases other than argon are commonly added to the ICP during laser ablation analyses. In particular $\mathrm{N}_{2}$ or $\mathrm{He}$ is used in the ICP center channel to improve the conversion of the 
analyte from ablated particle to atomic ion. These gases have also been shown to reduce the abundance of $\mathrm{MO}^{+}$interferences $[41,42]$ and, in the case of $\mathrm{He}$, improve transport out of the LA cell [43]. Using REE oxide ions as thermometric probe species, the effect of these gases on conditions in the ICP can be determined and used to give context to the enhancements produced in the spectrum. To accomplish this, the gas flow rate through the ablation cell was maintained constant at $0.5 \mathrm{~L} \mathrm{~min}^{-1}$ for each analysis to ensure that transport conditions from the ablation cell were consistant. The $\mathrm{M}^{+}$and $\mathrm{MO}^{+}$ion signals were then measured at a series of increasing Ar make-up gas flow rates, producing ion signal profiles similar to those found in Fig. 3a.

Changes in the measured ion signals relate to changes in the relative position of the various zones where $\mathrm{MO}^{+}, \mathrm{M}$ and $\mathrm{M}^{+}$species can be found relative to a fixed sampling position. This is a consequence of alterations in the gas flow rates, either for pure Ar or mixed gas flows [38,44-46]. Figure 4 illustrates the effect of the total gas flow rate on the position of a particular plasma zone, the initial radiation zone (IRZ), relative to the sampling orifice. At higher total flow rates, the IRZ is located closer to the sampler interface than when a lower total gas flow rate is used with a fixed sampling position [47].

All measurements were conducted at a fixed sampling position and power in this study. The sampling position cannot be easily adjusted for the ICP-MS instrument used in this study, as the radial and axial positions cannot be altered independently. The only option for obtaining axially-resolved results is to vary the aerosol gas flow rate, as described by Horlick et al. $[38,45]$. Variations in the plasma potential with changes in the gas flow rate should be minimal due to the use of a grounded shield with the ICP torch. Ion signal profiles such as those in Fig. 3a can thus be used as approximations to investigate axial spatial effects 
of gas identity and flow rates on the ICP through measurement of $\mathrm{M}^{+}$and $\mathrm{MO}^{+}$ions, although actual axially-resolved measurements would be better.

\section{$\mathrm{N}_{2}$ and Ar}

$\mathrm{N}_{2}$ has been used at low flow rates $\left(\sim 5\right.$ to $\left.100 \mathrm{~mL} \mathrm{~min}^{-1}\right)$ in the central gas flow by other investigators to increase sensitivity for atomic ions by factors of 2 to 5 , while reducing the $\mathrm{MO}^{+} / \mathrm{M}^{+}$signal ratio $[33,42,46,48-52] . \mathrm{MO}^{+} / \mathrm{M}^{+}$signal ratios for each of the elements from the REE disk at increasing $\mathrm{N}_{2}$ flow rates in the aerosol gas flow are shown in Fig. 5. The experiments are done by increasing the Ar make-up gas flow rate at each of the indicated $\mathrm{N}_{2}$ flow rates, with $0.5 \mathrm{~L} \mathrm{~min}^{-1}$ Ar through the ablation cell.

Fig. 5 shows that inclusion of $\mathrm{N}_{2}$ in the central channel lowers the minimum achievable $\mathrm{MO}^{+} / \mathrm{M}^{+}$ratio for all species observed, which is desirable. For $\mathrm{CeO}^{+}$, the most strongly-bound oxide investigated (Table $4[53,54]$ ), the $\mathrm{CeO}^{+} / \mathrm{Ce}^{+}$signal ratio decreased from $1.98 \times 10^{-3}$ without $\mathrm{N}_{2}$ to $3.26 \times 10^{-4}$ at the highest $\mathrm{N}_{2}$ flow rate. Similar behavior is found for all elements save Cs. Being the most weakly-bound of the polyatomic species analyzed, $\mathrm{CsO}^{+}\left(\mathrm{D}_{0}=3.04 \mathrm{eV}\right.$ [54]) was not abundant under all conditions investigated. It can thus be expected that polyatomic species with dissociation energies equal to or less than that of $\mathrm{CsO}^{+}$ will be present only at low abundance in a dry plasma. For the remainder of the species, the trend presented can be understood through the relationship between the $\mathrm{M}^{+}$and $\mathrm{MO}^{+}$signal profiles and the determined $\mathrm{T}_{\text {gas }}$ values. The behavior of $\mathrm{Y}^{+}$and $\mathrm{YO}^{+}$, as an established metric for plasma conditions, can be used to explore this phenomenon.

The $\mathrm{Y}^{+}$and $\mathrm{YO}^{+}$signal observed at four different flow rates of $\mathrm{N}_{2}$ in the central gas flow in Fig. 3 illustrates the effect of $\mathrm{N}_{2}$ on LA-ICP-MS analysis. Without $\mathrm{N}_{2}$, the central 
channel gas flow consists of $0.5 \mathrm{~L} \mathrm{~min}^{-1}$ of Ar from the ablation cell and an Ar make-up gas flow added after the ablation cell. The $\mathrm{Y}^{+}$signal maximum is at $\sim 0.9 \mathrm{~L} \mathrm{~min}^{-1}$ make-up gas flow, or $\sim 1.4 \mathrm{~L} \mathrm{~min}^{-1}$ total. This value is commonly seen on our instrument. Each $5 \mathrm{~mL}$ $\min ^{-1}$ increase in the added $\mathrm{N}_{2}$ shifts the $\mathrm{Y}^{+}$signal profile to lower make-up gas flows by $0.2 \mathrm{~L} \mathrm{~min}^{-1}$. The maximum $\mathrm{Y}^{+}$signal then occurs at lower gas flows, such that the maximum signal at $20 \mathrm{~mL} \mathrm{~min}^{-1}$ of added $\mathrm{N}_{2}$, and possibly at $10 \mathrm{~mL} \mathrm{~min}^{-1}$ of added $\mathrm{N}_{2}$, probably occurs at a make-up Ar flow below the investigated range. Rather than the $2 \mathrm{x}$ to $4 \mathrm{x}$ increase reported by others during LA-ICP-MS [42,52], we find little to no sensitivity enhancement with the addition of $\mathrm{N}_{2}$.

Despite the small gain in absolute signal for the atomic ion, an analytical advantage is still gained by introducing $\mathrm{N}_{2}$ into the ICP. At the Ar make-up gas flow rate corresponding to the $\mathrm{Y}^{+}$signal maximum, the abundance of $\mathrm{YO}^{+}$decreases as $\mathrm{N}_{2}$ flow rate increases (Fig. 3c). This trend is consistently observed for other $\mathrm{MO}^{+}$species (data not shown). By suppressing the polyatomic ion, accuracy and detection limits can be improved at $\mathrm{m} / \mathrm{z}$ values corresponding to the $\mathrm{MO}^{+}$interference.

The combined information on the atomic and polyatomic ions can be used to describe the effect of $\mathrm{N}_{2}$ in the ICP central channel through $\mathrm{T}_{\text {gas }}$ measurements. Compared to an Aronly plasma, $\mathrm{T}_{\text {gas }}$ is slightly greater at each make-up gas flow rate that yields the $\mathrm{M}^{+}$signal maximum, as shown by the points labeled with asterisks and arrows in Fig. $3 \mathrm{~d}$. $\mathrm{T}_{\text {gas }}$ also drops more extensively as the make-up gas is increased when $\mathrm{N}_{2}$ is present.

Various explanations have been offered by other researchers about the effects of $\mathrm{N}_{2}$ in the ICP. Hu et al. note that the thermal conductivity of $\mathrm{N}_{2}$ is $32 \mathrm{x}$ greater than that of Ar at ICP temperatures. Thus, energy transfer from gas to ablated sample particles within the axial 
channel could be better with a small dose of $\mathrm{N}_{2}$ in the Ar gas flow [42]. Conversely Sesi et al. observe that $\mathrm{N}_{2}$ addition to the aerosol gas flow cools the center channel of the plasma [33]. They propose that, as a molecular gas, the vibrational and rotational states of $\mathrm{N}_{2}$ dissipate heat transferred into the center of the ICP. However, when $\mathrm{N}_{2}$ is added to the auxiliary gas flow, an increase in the temperature of the center channel is observed. They attribute this effect to $\mathrm{N}_{2}$ facilitating an improved transfer of energy from the outer gas flow to the center channel of the plasma. Comparison of ion signal profiles collected by Agatemor and Beauchemin from an Ar-only plasma and a plasma with $\mathrm{N}_{2}$ added to a sheath gas flow indicate the plasma shrinks when $\mathrm{N}_{2}$ is introduced [55]. The formation of strongly-bound $\mathrm{NO}^{+}\left(\mathrm{D}_{0}=11.76 \mathrm{eV}\right)$ would also effectively bind the $\mathrm{O}$ atoms present in the ICP and prevent the formation of $\mathrm{MO}^{+}$species from analyte ions [42].

Based on the response of $\mathrm{T}_{\text {gas }}$ to changes in the gas flows, it appears to be a combination of these factors that best describes the effect of $\mathrm{N}_{2}$ in a dry plasma. The increasingly extensive reduction in $\mathrm{T}_{\text {gas }}$ across the range of Ar flows as more $\mathrm{N}_{2}$ is introduced supports both the $\mathrm{N}_{2}$ shrinking the normal analytical zone (NAZ) in the plasma as well as acting as an energy sink. Photographic studies of the plasma during ablation experiments have shown that the regions over which atomization and ionization occur are more diffuse and ill-defined compared to the ICP during solution introduction [47]. The plasma shrinkage induced by the addition of $\mathrm{N}_{2}$ to the aerosol gas flow compacts these zones. A better defined and thinner NAZ decreases the radial diffusion of ions, increasing the proportion of ions positioned in line with the extraction interface at lower Ar flow rates. Fig. 3a shows that a similar result can be achieved when operating at low gas flow rates by increasing the aerosol gas flow in an Ar-only plasma, which also decreases diffusion to the 
boundaries of the center channel of the ICP. By comparison, the introduction of $\mathrm{N}_{2}$ causes the optimum NAZ position, where the atomic signal is maximized, to be reached at lower central gas flows. With a modest amount of $\mathrm{N}_{2}$ present, a similar value of $\mathrm{T}_{\text {gas }}$ is observed as at lower Ar flow rates. This can prove analytically beneficial in several ways. Operation at lower total aerosol gas flows can reduce fractionation [43] and, as shown in Fig. 3, reduce the formation of polyatomic ions in the ICP.

\section{He and Ar}

While $\mathrm{N}_{2}$ is used as a complement to Ar, He is employed as a direct substitute for $\mathrm{Ar}$ in LA-ICP-MS analyses to enhance signal through processes that occur during ablation and transport as well as in the ICP $[33,43,56]$. To investigate the effect of $\mathrm{He}$ on $\mathrm{M}^{+}$and $\mathrm{MO}^{+}$ signal profiles, a flow rate of $0.5 \mathrm{~L} \mathrm{~min}^{-1} \mathrm{He}$ only was passed through the ablation cell to provide constant ablation and transport conditions. Variable amounts of Ar were then added to the aerosol gas flow before introduction into the ICP. A fixed flow rate for He was maintained for all analyses. Again, this situation corresponds to common analytical practice.

For the measured $\mathrm{Y}^{+}$signal (Fig. 6a), replacing Ar with He resulted in a 50\% increase in the $\mathrm{Y}^{+}$signal maximum over an Ar-only plasma at the "best" Ar flow rate for each case. This is only achieved by re-optimizing plasma conditions. Simply substituting He for Ar at the "best" Ar make-up gas flow rate $\left(0.9 \mathrm{~L} \mathrm{~min}^{-1}\right)$ would otherwise result in a nearly $50 \%$ decrease in $\mathrm{M}^{+}$ion abundance.

An interesting difference between the He-Ar and Ar-only plasmas can be found in the $\mathrm{YO}^{+}$signal profile (Fig. 6b) and the attendant $\mathrm{T}_{\text {gas }}$ values (Fig. 6c) measured from the $\mathrm{YO}^{+} / \mathrm{Y}^{+}$ratio, especially at high Ar make-up gas flow rates. In the Ar-only plasma, $\mathrm{YO}^{+}$ 
abundance increased $13 \mathrm{x}$ from the optimum make-up gas flow $\left(0.9 \mathrm{~L} \mathrm{~min}^{-1}\right)$ to the highest setting investigated. Over the same range of settings in the He-Ar plasma $\mathrm{YO}^{+}$increased only $\sim 4 \mathrm{x}$. The corresponding $\mathrm{T}_{\text {gas }}$ values also show less variation with make-up gas flow rate. The He-Ar plasma maintained a $\mathrm{T}_{\text {gas }}$ of $\sim 5000 \mathrm{~K}$ while the Ar-only plasma experienced a drop of nearly $1000 \mathrm{~K}$ in calculated $\mathrm{T}_{\text {gas }}$ over the examined range of gas settings.

For Fig. 7a there is He through the ablation cell with Ar make-up gas, but no $\mathrm{N}_{2}$. Similar ratios are observed at low make-up gas flow rates as shown for the Ar-only plasma in Fig. 5a. Unlike the Ar-only plasma, however, these ratios do not change much even when the make-up gas flow rate is increased. With He through the ablation cell $\mathrm{T}_{\text {gas }}$ remains high, even at high total gas flows into the central channel. If high aerosol gas flows are necessary for an analysis, incorporation of He in the center channel of the ICP can be used to minimize the abundance of $\mathrm{MO}^{+}$interferences.

These results corroborate other findings on the effect of $\mathrm{He}$ in the plasma [43]. At $\sim 5000 \mathrm{~K}$, He has a thermal conductivity of $\sim 1 \mathrm{~W} \mathrm{~m}^{-1} \mathrm{~K}^{-1}$ [57], much greater than that of Ar $\left(\sim 0.1 \mathrm{~W} \mathrm{~m}^{-1} \mathrm{~K}^{-1}\right)$ [58]. It has been suggested that a small dose of He allows for improved transfer of energy from both the induction region into the axial channel and within the axial channel itself [33]. The increase in maximum achievable $\mathrm{Y}^{+}$signal with He introduction (Fig. 6a) due to more efficient vaporization, ionization and atomization of the ablated material is one expression of this attribute. In addition, better energy transfer from the bulk plasma enables the ICP to attain a consistent $\mathrm{T}_{\text {gas }}$ throughout the center channel. As a result the measured $\mathrm{T}_{\text {gas }}$ does not change much with alterations in the sampling position of the HeAr plasma induced by changes in the gas load (Fig. 6c) over a very wide range (0.4 to $1.3 \mathrm{Ar}$ make-up gas flow rate), suppressing the $\mathrm{YO}^{+}$signal at locations upstream in the ICP. This 
permits a greater freedom when tuning the gas settings for optimal signal, which may occur at different central gas flow rates for different samples or even different analyte elements, without sacrificing the enhanced figures of merit obtained by reduction of polyatomic interferences.

\section{$\mathbf{N}_{2}$, He and Ar}

In further experiments, $\mathrm{N}_{2}$ was added to the He-Ar plasma to establish if an analytical advantage could be gained by combining two additives. The variation of $\mathrm{MO}^{+} / \mathrm{M}^{+}$ratios with the addition of $\mathrm{N}_{2}$ to a He-Ar plasma, presented in Fig. $7 \mathrm{~b}, \mathrm{c}$, and d, is similar to that of the Ar-only plasma. Lower ratios are reached for each species as the $\mathrm{N}_{2}$ flow rate increases. At higher make-up gas flow rates (Fig. $7 \mathrm{c}$ and d) the $\mathrm{MO}^{+} / \mathrm{M}^{+}$ratios change more than in the absence of $\mathrm{N}_{2}$ (Fig. 7a).

The combined influences of $\mathrm{He}$ and $\mathrm{N}_{2}$ on the ICP are more clearly evident in the signal profiles of the atomic and oxide ions and the resultant $\mathrm{T}_{\text {gas }}$ values. Fig. 8a shows that the introduction of $\mathrm{N}_{2}$ shifts the $\mathrm{Y}^{+}$signal maximum to lower make-up gas flows, much like in Fig. 3a for the Ar-only plasma. Raising the $\mathrm{N}_{2}$ flow rate above the initial addition of $5 \mathrm{~mL}$ $\min ^{-1}$ provides diminishing changes in the optimum make-up gas setting, with He again moderating the effect of increases in gas load. A small increase in $\mathrm{Y}^{+}$sensitivity is realized only at a $\mathrm{N}_{2}$ flow of $10 \mathrm{~mL} \mathrm{~min}^{-1}$ and at a lesser extent than when added to the Ar-only plasma. The moderating effect of He was also observed in the overall signal profile at each gas setting. Compared to the rate of decline presented at higher Ar make-up flow rates in the $\mathrm{N}_{2}$-Ar plasma (Fig. 3a), the $\mathrm{Y}^{+}$signal experienced a more gradual drop past peak signal (Fig. 8a). The abundance of $\mathrm{YO}^{+}$(Fig. 8b) is similar for all added amounts of $\mathrm{N}_{2}$ at lower $\mathrm{Ar}$ 
make-up gas flow rates. At low make-up gas flow rates the $\mathrm{YO}^{+}$signals do not increase much, while the $\mathrm{Y}^{+}$signal does increase, so the measured $\mathrm{T}_{\text {gas }}$ values (Fig. 8c) are moderately higher with $\mathrm{N}_{2}$ introduced into the He-Ar plasma. These $\mathrm{T}_{\text {gas }}$ values are hotter than those measured when $\mathrm{N}_{2}$ was added to the Ar-only plasma (Fig. 3d) and remain elevated at higher make-up gas rates (up to $1.0 \mathrm{~L} \mathrm{~min}^{-1}$ ). Past this threshold, decreased $\mathrm{Y}^{+}$and increased $\mathrm{YO}^{+}$ abundances mean $\mathrm{T}_{\text {gas }}$ drops to values below those reached in the exclusively He-Ar ICP.

The combined effects of $\mathrm{N}_{2}$ and $\mathrm{He}$ in the center channel of the ICP could prove favorable if managed properly. The hotter $\mathrm{T}_{\text {gas }}$ resulting from the greater thermal conductivity of $\mathrm{He}$ is sustained even in the presence of the temperature dampening effect caused by $\mathrm{N}_{2}$. Similarly the compaction of the $\mathrm{NAZ}$ by $\mathrm{N}_{2}$ can still be attained in a $\mathrm{He}$ environment, providing all the advantages of operation at lower total gas loads. $\mathrm{T}_{\text {gas }}$ at maximum signal for $\mathrm{Y}^{+}$when $\mathrm{N}_{2}$ is included in the He-Ar plasma is moderately higher than in all other investigated central channel gas combinations as presented in Table 5.

Careful tuning of the flow rates for the various gases can produce optimal atomic ion signal for a LA-ICP-MS analysis while keeping polyatomic ion interferences to an acceptable minimum. Possible gains are lost if the $\mathrm{N}_{2}$ flow rate is set too high, however. Presumably, the tip of the sampling cone is now upstream of the NAZ in the ICP. Though the plasma does not cool as quickly in these positions due to the presence of He in the center channel, the benefit of $\mathrm{N}_{2}$ addition is lost as $\mathrm{T}_{\text {gas }}$ drops below the value that would be achieved with He and Ar alone. As they are among the most strongly bound of the polyatomic interferences encountered in the ICP, careful management of oxide-based polyatomics with mixed gas plasmas should help ensure that a breadth of polyatomic interferences are minimized throughout the spectrum. 


\section{Conclusions}

Comparison of $\mathrm{MO}^{+}$interferences between the wet plasma conditions found in solution nebulization ICP-MS and the dry plasma conditions of LA-ICP-MS reveals the changed environment in the ICP directly impacts the abundance of $\mathrm{MO}^{+}$ions in the spectrum. The decreased availability of oxygen reduces the abundance of oxide ions for both silicate and metallic matrices. While the oxide ions studied here are less prevalent in LAICP-MS, interferences from $\mathrm{MO}^{+}$ions from matrix and major elements can still arise. Ablation of silicate samples results in $\mathrm{T}_{\text {gas }}$ values similar to those found in solution nebulization. Apparently, the ICP can sufficiently atomize these particles in the aerosol. This may be less true for metallic samples, where ablation of this sample type often produces larger particles $[59,60]$ that cannot be completely atomized in the plasma. However, the minimal oxygen content of metallic samples prevents an increase in the abundance of $\mathrm{MO}^{+}$ ions in the mass spectrum from these large particles.

In LA-ICP-MS, mixed gas plasmas provide a way to reduce $\mathrm{MO}^{+}$ion interferences without large sacrifices in the abundance of $\mathrm{M}^{+}$ions. The addition of $\mathrm{N}_{2}$ to an Ar aerosol gas flow produces a narrower NAZ in the plasma, which can be sampled at lower total gas flows. Operation under these conditions increases $\mathrm{T}_{\text {gas }}$, calculated by monitoring the $\mathrm{YO}^{+} / \mathrm{Y}^{+}$ratio, by reducing polyatomic ion levels in the spectrum. This effect is compounded when He is included in the aerosol flow at the ablation cell. Helium added to an Ar plasma increases $\mathrm{T}_{\text {gas }}$ and enhances sensitivity for atomic ions through a longer zone in the center channel of the ICP. When both $\mathrm{He}$ and $\mathrm{N}_{2}$ are added, a modest $20 \%$ sensitivity enhancement is gained while polyatomics are suppressed even further, courtesy of plasma operation at a lower total 
aerosol gas flow rate. The highest operating $\mathrm{T}_{\text {gas }}$ is achieved with this combination, providing the best analytical performance observed.

Future work could focus on the formation of $\mathrm{MO}^{+}$ion interferences from other types of matrices. Ablation of biological materials is of increasing interest and represents a sample type not investigated here. Study of the polyatomics produced from ablation of the organic, and often water-rich, matrices encountered in these samples may provide valuable information to improve the figures of merit for this growing area of LA-ICP-MS analysis [61-63].

\section{Acknowledgements}

This research was supported by the National Science Foundation (Award No. CHE0309381) through the Institute for Physical Research and Technology at ISU. The ELEMENT 1 ICP-MS was purchased with funds provided by the U. S. Department of Energy, Office of Nuclear Nonproliferation (NA-22) and the Office of Basic Energy Sciences. The authors thank Dr. Scott Schlorholtz for preparing the silicate sample for analysis, and Larry Jones and Arne Swanson for providing the Y metal sample. The Ames Laboratory is operated for the U. S. Department of Energy by Iowa State University under Contract No. DEAC02-07CH11358.

\section{References}

[1] R.S. Houk, V.A. Fassel, G.D. Flesch, H.J. Svec, A.L. Gray, C.E. Taylor, Inductively coupled argon plasma as an ion source for mass spectrometric determination of trace elements, Anal. Chem. 52 (1980) 2283-2289. 
[2] K.E. Jarvis, A.L. Gray, R.S. Houk, Handbook of Inductively Coupled Plasma Mass Spectrometry (Chapman and Hall, New York, 1992).

[3] N. Jakubowski, Analytical plasma ion sources for elemental mass spectrometry: where are we coming from-where are we going to?, J. Anal. Atom. Spectrom. 23 (2008) 673-684.

[4] C.B. Douthitt, Commercial development of HR-ICPMS, MC-ICPMS and HRGDMS, J. Anal. Atom. Spectrom. 23 (2008) 685-689.

[5] J.S. Becker, G. Seifert, A.I. Saprykin, H.-J. Dietze, Mass Spectrometric and Theoretical Investigations Into the Formation of Argon Molecular Ions in Plasma Mass Spectrometry, J. Anal. Atom. Spectrom. 11 (1996) 643-648.

[6] H. Niu, R.S. Houk, Fundamental aspects of ion extraction in inductively coupled plasma mass spectrometry, Spectrochim. Acta Part B 51 (1996) 779-815.

[7] V.N. Epov, D. Lariviere, E.N. Epova, R.D. Evans, Polyatomic Interferences Produced by Macroelements During Direct Multi-Elemental ICP-MS Hydrochemical Analysis, Geostand. Geoanal. Res. 28 (2004) 213-224.

[8] U. Nygren, H. Ramebäck, D.C. Baxter, C. Nilsson, Lanthanide phosphate interferences in actinide determination using inductively coupled plasma mass spectrometry, J. Anal. Atom. Spectrom. 20 (2005) 529-534.

[9] A.P. Krushevska, Y. Zhou, V. Ravikumar, Y.-J. Kim, J. Hinrichs, Chromium based polyatomic interferences on rhodium in ICP-MS, J. Anal. Atom. Spectrom. 21 (2006) 847-855.

[10] S.J. Jiang, R.S. Houk, M.A. Stevens, Alleviation of overlap interferences for determination of potassium isotope ratios by inductively coupled plasma mass spectrometry, Analytical Chemistry 60 (1988) 1217-1221.

[11] S.D. Tanner, Characterization of ionization and matrix suppression in inductively coupled 'cold' plasma mass spectrometry, Journal of Analytical Atomic Spectrometry 10 (1995) 905-921.

[12] J.T. Rowan, R.S. Houk, Attenuation of Polyatomic Ion Interferences in Inductively Coupled Plasma Mass Spectrometry by Gas-Phase Collisions, Appl. Spectrosc. 43 (1989) 976-980.

[13] B. Hattendorf, D. Günther, Characteristics and capabilities of an ICP-MS with a dynamic reaction cell for dry aerosols and laser ablation, J. Anal. Atom. Spectrom. 15 (2000) 1125-1131.

[14] P.R.D. Mason, W.J. Kraan, Attenuation of spectral interferences during laser ablation inductively coupled plasma spectrometry (LA-ICP-MS) using an rf only collision and reaction cell, J. Anal. Atom. Spectrom. 17 (2002) 858-867.

[15] D. Pick, M. Leiterer, J.W. Einax, Reduction of polyatomic interferences in biological material using dynamic reaction cell ICP-MS, Microchem. J. 95 (2010) 315-319.

[16] M.A. Amr, A.M. Abdel-Lateef, Comparing the capability of collision/reaction cell quadrupole and sector field inductively coupled plasma mass spectrometers for interference removal from ${ }^{90} \mathrm{Sr},{ }^{137} \mathrm{Cs}$ and ${ }^{226} \mathrm{Ra}$, Int. J. Mass Spectrom. 299 (2011) 184-190.

[17] J.L.M. de Boer, Possibilities and limitations of spectral fitting to reduce polyatomic ion interferences in inductively coupled plasma quadrupole mass spectrometry in the mass range 51-88, Spectrochim. Acta Part B 52 (1997) 389-403. 
[18] X. Cao, M. Yin, X. Wang, Elimination of the spectral interference from polyatomic ions with rare earth elements in inductively coupled plasma mass spectrometry by combining algebraic correction with chromatographic separation, Spectrochim. Acta Part B 56 (2001) 431-441.

[19] N.M. Raut, L.-S. Huang, S.K. Aggarwal, K.-C. Lin, Mathematical Correction for Polyatomic Isobaric Spectral Interferences in Determination of Lanthanides by Inductively Coupled Plasma Mass Spectrometry, J. Chin. Chem. Soc-Taip. 52 (2005).

[20] F. Laborda, M. Górriz, E. Bolea, J.R. Castillo, Mathematical correction for polyatomic interferences in the speciation of chromium by liquid chromatographyinductively coupled plasma quadrupole mass spectrometry, Spectrochim. Acta Part B 61 (2006) 433-437.

[21] N.M. Reed, R.O. Cairns, R.C. Hutton, Y. Takaku, Characterization of Polyatomic Ion Interferences in Inductively Coupled Plasma Mass Spectrometry Using a High Resolution Mass Spectrometer, J. Anal. Atom. Spectrom. 9 (1994) 881-884.

[22] C. Pickhardt, H.-J. Dietze, J.S. Becker, Laser ablation inductively coupled plasma mass spectrometry for direct isotope ratio measurements on solid samples, Int. J. Mass Spectrom. 242 (2005) 273-280.

[23] R.S. Houk, N. Praphairaksit, Dissociation of polyatomic ions in inductively coupled plasma, Spectrochim. Acta Part B 56 (2001) 1069-1096.

[24] N.F. Zahran, A.I. Helal, M.A. Amr, A. Abdel-Hafiez, H.T. Mohsen, Formation of polyatomic ions from the skimmer cone in inductively coupled plasma mass spectrometry, Int. J. Mass Spectrom. 226 (2003) 271-278.

[25] J.W. Ferguson, R.S. Houk, High Resolution studies of the origins of polyatomic ions in inductively coupled plasma-mass spectrometry, Part I. Identification methods and effects of neutral gas density assumptions, extraction voltage, and cone material, Spectrochimica Acta Part B 61B (2006) 905-915.

[26] J.W. Ferguson, T.J. Dudley, K.C. Sears, S.M. McIntyre, M.S. Gordon, R.S. Houk, Polyatomic ions in inductively coupled plasma-mass spectrometry, Spectrochimica Acta Part B 64B (2009) 690-696.

[27] S.M. McIntyre, J.W. Ferguson, T.M. Witte, R.S. Houk, Measurement of gas kinetic temperatures for polyatomic ions in inductively coupled plasma-mass spectrometry: Validation and refinements, Spectrochimica Acta Part B 66 (2011) 248-254.

[28] A.J.R. Kent, C.A.A. Ungerer, Production of barium and light rare earth element oxides during LA-ICP-MS microanalysis, J. Anal. Atom. Spectrom. 20 (2005) 12561262.

[29] D.C. Perdian, S.J. Bajic, D.P. Baldwin, R.S. Houk, Time-resolved studies of particle effects in laser ablation inductively coupled plasma-mass spectrometry Part 2. Investigation of $\mathrm{MO}+$ ions, effect of sample morphology, transport gas, and binding agents, J. Anal. Atom. Spectrom. 23 (2008) 336-341.

[30] M. Guillong, L. Danyushevsky, M. Walle, M. Raveggi, The effect of quadrupole ICPMS interface and ion lens design on argide formation. Implications for LAICPMS analysis of PGE's in geological samples, J. Anal. Atom. Spectrom. 26 (2011) 1401-1407.

[31] A.L. Gray, Solid Sample Introduction by Laser Ablation for Inductively Coupled Plasma Source Mass Spectrometry, Analyst 110 (1985) 551-556. 
[32] B. Hattendorf, C. Latkoczy, D. Günther, Laser ablation-ICPMS, Anal. Chem. 75 (2003) 341A-347A.

[33] N.N. Sesi, A. MacKenzie, K.E. Shanks, P. Yang, G.M. Hieftjie, Fundamental studies of mixed-gas inductively coupled plasmas, Spectrochim. Acta Part B 49 (1994) 12591282.

[34] H.P. Longerich, Mass Spectrometric Determination of the Temperature of an Argon Inductively Coupled Plasma From the Formation of the Singly Charged Monoxide Rare Earths and their Known Dissociation Energies, J. Anal. Atom. Spectrom. 4 (1989) 491-497.

[35] E.H. Evans, L. Ebdon, L. Rowley, Comparative study of the determination of equilibrium dissociation temperature in inductively coupled plasma-mass spectrometry, Spectrochim. Acta Part B 57 (2002) 741-754.

[36] K.P. Huber, G. Herzberg, Molecular Spectra and Molecular Structure IV. Constants of Diatomic Molecules (Van Nostrand Reinhold, New York, 1979).

[37] C.P. Ingle, B.L. Sharp, M.S.A. Horstwood, R.R. Parrish, D.J. Lewis, Instrument response functions, mass bias, and matrix effects in isotope ratio measurements and semi-quantitative analysis by single and multi-collectore ICP-MS, J. Anal. Atom. Spectrom. 18 (2003) 219-229.

[38] M.A. Vaughan, G. Horlick, Oxide, Hydroxide and Doubly-Charged Analyte Species in Inductively Coupled Plasma/Mass Spectrometry, Appl. Spectrosc. 40 (1986) 434445.

[39] M.G. Minnich, R.S. Houk, Comparison of cryogenic and membrane desolvation for attenuation of oxide, hydride and hydroxide ions and ions containing chlorine in inductively coupled plasma mass spectrometry, J. Anal. Atom. Spectrom. 13 (1998) 167-174.

[40] C. Liu, S.S. Mao, X. Zeng, R. Greif, R.E. Russo, Nanosecond and Femtosecond Laser Ablation of Brass Particulate and ICPMS Measurements, Anal. Chem. 76 (2004) 379-383.

[41] S.F. Durrant, Feasability of improvement in analytical performance in laser ablation inductively coupled plasma-mass spectrometry (LA-ICP-MS) by addition of nitrogen to the argon plasma, Fresenius' J. Anal. Chem. (1994) 768-771.

[42] Z. Hu, S. Gao, Y. Liu, S. Hu, H. Chen, H. Yuan, Signal enhancement in laser ablation ICP-MS by addition of nitrogen in the central channel gas, J. Anal. Atom. Spectrom. 23 (2008) 1093-1101.

[43] Z. Wang, B. Hattendorf, D. Günther, Analyte Response in Laser Ablation Inductively Coupled Plasma Mass Spectrometry, J. Am. Soc. Mass Spectrom. 17 (2006) 641-651.

[44] S.R. Koirtyohann, J.S. Jones, D.A. Yates, Nomenclature system for the low-power argon inductively coupled plasma, Anal. Chem. 52 (1980) 1965-1966.

[45] M.A. Vaughan, G. Horlick, S.H. Tan, Effect of Operating Parameters on Analyte Signals in Inductively Coupled Plasma-Mass Spectrometry, J. Anal. Atom. Spectrom. 2 (1987) 765-772.

[46] J.W.H. Lam, G. Horlick, A comparison of argon and mixed gas plasmas for inductively coupled plasma-mass spectrometry, Spectrochim. Acta Part B 45 (1990) 1313-1325. 
[47] D.B. Aeschliman, S.J. Bajic, D.P. Baldwin, R.S. Houk, High-speed digital photographic study of an inductively coupled plasma during laser ablation: comparison of dried aerosols from a microconcentric nebulizer and solid particles from laser ablation, J. Anal. Atom. Spectrom. 18 (2003) 1008-1014.

[48] J. Wang, E.H. Evans, J.A. Caruso, Addition of Molecular Gases to Argon Gas Flows for the Reduction of Polyatomic-ion Interferences in Inductively Coupled Plasma Mass Spectrometry, J. Anal. Atom. Spectrom. 7 (1992) 929-936.

[49] R.W. Nesbitt, T. Hirata, I.B. Butler, J.A. Milton, UV Laser Ablation ICP-MS: Some Applications in the Earth Sciences, Geostandard. Newslett. 20 (1997) 231-243.

[50] T. Iizuka, T. Hirata, Improvements of precision and accuracy in in situ Hf isotope microanalysis of zircon using the laser ablation-MC-ICPMS technique, Chem. Geol. 220 (2005) 121-137.

[51] M. Guillong, C.A. Heinrich, Sensitivity enhancement in laser ablation ICP-MS using small amounts of hydrogen in the carrier gas, J. Anal. Atom. Spectrom. 22 (2007) 1488-1494.

[52] M. Shaheen, B.J. Fryer, Improving the analytical capabilities of femtosecond laser ablation multicollector ICP-MS for high precision $\mathrm{Pb}$ isotopic analysis: the role of hydrogen and nitrogen, J. Anal. Atom. Spectrom. (2010).

[53] K. Schofield, An Overlooked Series of Gas Phase Diatomic Metal Oxide Ions that Are Long-Lived, J. Phys. Chem. A 110 (2006) 6938-6947.

[54] CRC Handbook of Chemistry and Physics, 92nd Edn. (CRC Press, Boca Raton, FL, 2011).

[55] D. Agatemor, D. Beauchemin, Towards the reduction of matrix effects in inductively coupled plasma mass spectrometry without compromising detection limits: The use of argon-nitrogen mixed-gas plasma, Spectrochim. Acta Part B 66 (2011) 1-11.

[56] D. Günther, C.A. Heinrich, Enhanced sensitivity in laser ablation-ICP mass spectrometry using helium-argon mixtures as aerosol carrier Plenary Lecture, J. Anal. Atom. Spectrom. 14 (1999) 1363-1368.

[57] A.B. Murphy, Transport Coefficients of Helium and Argon-Helium Plasmas, IEEE T. Plasma Sci. 25 (1997) 809-814.

[58] Y.Q. Tang, C. Trassy, Inductively coupled plasma: the role of water in axial excitation temperatures, Spectrochim. Acta Part B 41 (1986) 143-150.

[59] J. González, C. Liu, X. Mao, R.E. Russo, UV-femtosecond laser ablation-ICP-MS for analysis of alloy samples, J. Anal. Atom. Spectrom. 19 (2004) 1165-1168.

[60] D.B. Aeschliman, S.J. Bajic, D.P. Baldwin, R.S. Houk, Spatially-resolved analysis of solids by laser ablation-inductively coupled plasma-mass spectrometry: trace elemental quantification without matrix-matched solid standards, J. Anal. Atom. Spectrom. 18 (2003) 872-877.

[61] S.F. Durrant, N.I. Ward, Recent biological and environmental applications of laser ablation inductively coupled plasma mass spectrometry (LA-ICP-MS), Journal of Analytical Atomic Spectrometry 20 (2005) 821-829.

[62] M.V. Zoriy, M. Kayser, A. Izmer, C. Pickhardt, J.S. Becker, Determination of uranium isotopic ratios in biological samples using laser ablation inductively coupled plasma double focusing sector field mass spectrometry with cooled ablation chamber, International Journal of Mass Spectrometry 242 (2005) 297-302. 
[63] J.S. Becker, M. Zoriy, J.S. Becker, J. Dobrowolska, A. Matusch, Laser ablation inductively coupled plasma mass spectrometry (LA-ICP-MS) in elemental imaging of biological tissues and in proteomics, Journal of Analytical Atomic Spectrometry 22 (2007) 736-744. 
Table 1. Spectroscopic constants for $\mathrm{YO}^{+}$( $\omega$ and $B$ are for the neutral species)

\begin{tabular}{ccccc}
$D_{0}(\mathrm{eV})$ & $\omega\left(\mathrm{cm}^{-1}\right)$ & $B\left(\mathrm{~cm}^{-1}\right)$ & $\mathrm{g}$ & Refs \\
\hline 7.24 & 861 & 0.3881 & 1 & {$[35,36]$} \\
\hline
\end{tabular}

Table 2. ICP-MS Operating Parameters

\begin{tabular}{|c|c|}
\hline ICP-MS Instrument & Thermo Finnigan ELEMENT 1 \\
\hline \multirow[t]{2}{*}{ Resolution } & Low $(\mathrm{m} / \Delta \mathrm{m}=300)$ \\
\hline & $\operatorname{Medium}(\mathrm{m} / \Delta \mathrm{m}=4000)$ \\
\hline Torch & Shielded (grounded Pt guard electrode) \\
\hline RF Power & $1200 \mathrm{~W}$ \\
\hline Outer Gas & $6 \mathrm{~L} \mathrm{~min}^{-1}$ \\
\hline Auxiliary Gas & $0.9 \mathrm{~L} \mathrm{~min}^{-1}$ \\
\hline \multicolumn{2}{|l|}{ Aerosol Gas } \\
\hline Ar-only plasma & $0.925-1.275 \mathrm{~L} \mathrm{~min}^{-1} \mathrm{Ar}$ \\
\hline Ar and $\mathrm{N}_{2}$ plasma & $0.5 \mathrm{~L} \mathrm{~min}^{-1} \mathrm{Ar}$ (ablation sweep gas) \\
\hline \multirow{5}{*}{$\mathrm{He}-\mathrm{Ar}$ and $\mathrm{N}_{2}$ plasma } & $0.4-1.3 \mathrm{~L} \mathrm{~min}^{-1} \mathrm{Ar}$ (make-up gas) \\
\hline & $0-20 \mathrm{~mL} \min ^{-1} \mathrm{~N}_{2}$ \\
\hline & $0.5 \mathrm{~L} \mathrm{~min}^{-1} \mathrm{He}$ (ablation sweep gas) \\
\hline & $0.4-1.3 \mathrm{~L} \mathrm{~min}^{-1} \mathrm{Ar}$ (make-up gas) \\
\hline & $0-20 \mathrm{~mL} \mathrm{~min}-1 \mathrm{~N}_{2}$ \\
\hline Detector Mode & Dual (analog and pulse counting) \\
\hline Laser System & CETAC Technologies LSX-500 Nd:YAG 266 nm \\
\hline Pulse Energy & $8.65 \mathrm{~mJ}$ \\
\hline Spot Size & $150 \mu \mathrm{m}$ \\
\hline Repetition Rate & $10 \mathrm{~Hz}$ \\
\hline Ablation Mode & Single spot \\
\hline
\end{tabular}


Table 3. Comparison of $\mathrm{MO}^{+}$abundance from silicate (Y-doped glass) and metallic ( $\mathrm{Y}$ metal) samples during both solution nebulization and LA-ICP-MS

(All data corrected for mass bias)

\begin{tabular}{lccc} 
& $\begin{array}{c}\mathrm{YO}^{+} / \mathrm{Y}^{+} \\
\text {Signal } \\
\text { Ratios }\end{array}$ & $\begin{array}{c}\mathrm{T}_{\text {gas }} \\
(\mathrm{K})\end{array}$ & $\begin{array}{c}\mathrm{n}_{\mathrm{O}} \\
\left(\mathrm{cm}^{-3}\right)\end{array}$ \\
\hline $\begin{array}{l}\text { Solution } \\
\text { Nebulization }\end{array}$ & $1.67 \times 10^{-2}$ & 5630 & $9.41 \times 10^{16}$ \\
$\begin{array}{l}\text { Laser } \\
\left.\begin{array}{l}\text { Ablation } \\
(\mathrm{Y} \text { in SiO }\end{array}\right)\end{array}$ & $1.36 \times 10^{-4}$ & 5230 & $1.81 \times 10^{14}$ \\
$\begin{array}{l}\text { Laser } \\
\begin{array}{l}\text { Ablation } \\
(\mathrm{Y} \text { metal })\end{array}\end{array}$ & $1.43 \times 10^{-4}$ & 5210 & $1.81 \times 10^{14}$ \\
\hline
\end{tabular}

Table 4. Dissociation energies of $\mathrm{MO}^{+}$(All Ref [53] except $\mathrm{CsO}^{+}$[54])

$\left(\mathrm{D}_{0}\left(\mathrm{CsO}^{+}\right)=\mathrm{D}_{0}(\mathrm{CsO})+\mathrm{IE}(\mathrm{Cs})-\mathrm{IE}(\mathrm{CsO})\right.$

\begin{tabular}{cc}
$\mathrm{MO}^{+}$ & $\begin{array}{c}\mathrm{D}_{0} \\
(\mathrm{eV})\end{array}$ \\
\hline $\mathrm{CeO}^{+}$ & 8.83 \\
$\mathrm{TaO}^{+}$ & 7.89 \\
$\mathrm{TbO}^{+}$ & 7.71 \\
$\mathrm{TmO}^{+}$ & 4.90 \\
$\mathrm{CsO}^{+}$ & 0.57 \\
\hline
\end{tabular}

Table 5. $\mathrm{T}_{\text {gas }}$ values at the maximum $\mathrm{Y}^{+}$signal for each aerosol gas combination.
(a) Ar: $0.9 \mathrm{~L} \mathrm{~min}^{-1} \mathrm{Ar}$
(b) $\mathrm{N}_{2}$-Ar: $5 \mathrm{~mL} \mathrm{~min}^{-1} \mathrm{~N}_{2}, 0.4 \mathrm{~L} \mathrm{~min}^{-1} \mathrm{Ar}$
(c) He-Ar: $0.5 \mathrm{~L} \mathrm{~min}^{-1} \mathrm{He}, 1.1 \mathrm{~L} \mathrm{~min}^{-1} \mathrm{Ar}$
(d) $\mathrm{N}_{2}$-He-Ar: $20 \mathrm{~mL} \mathrm{~min}^{-1} \mathrm{~N}_{2}, 0.5 \mathrm{~L} \mathrm{~min}^{-1} \mathrm{He}, 0.6 \mathrm{~L} \mathrm{~min}^{-1} \mathrm{Ar}$

\begin{tabular}{|c|c|c|c|c|}
\hline Gas Mixture & a) $\mathrm{Ar}$ & b) $\mathrm{N}_{2}-\mathrm{Ar}$ & c) $\mathrm{He}-\mathrm{Ar}$ & d) $\mathrm{N}_{2}-\mathrm{He}-\mathrm{Ar}$ \\
\hline $\begin{array}{c}T_{\text {gas }}(\mathrm{K}) \\
\mathrm{Y}^{+} \text {Signal }\left(\mathrm{C} \mathrm{s}^{-1}\right)\end{array}$ & $\begin{array}{c}5040 \\
2.27 \times 10^{8}\end{array}$ & $\begin{array}{c}5380 \\
2.37 \times 10^{8}\end{array}$ & $\begin{array}{c}4930 \\
3.29 \times 10^{8}\end{array}$ & $\begin{array}{c}5810 \\
3.86 \times 10^{8}\end{array}$ \\
\hline
\end{tabular}



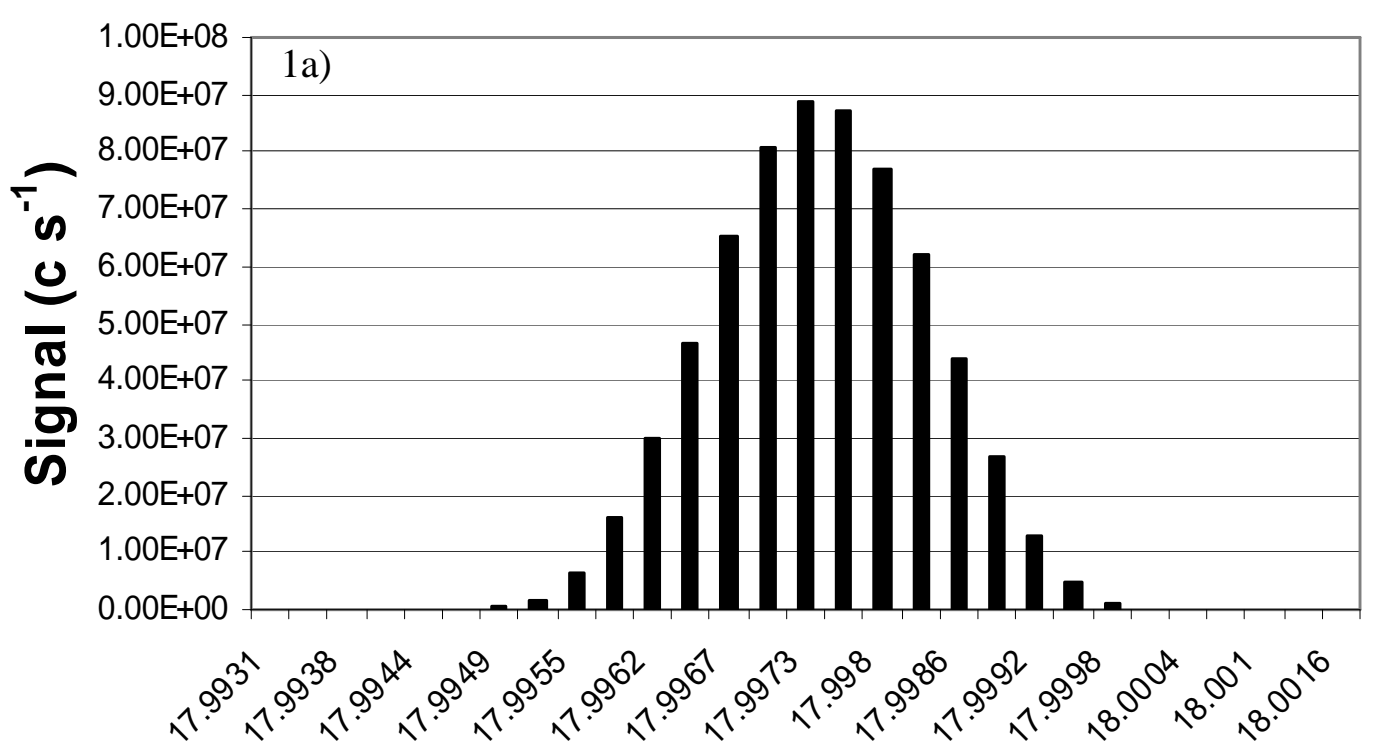

$\mathrm{m} / \mathbf{z}$ (Da)

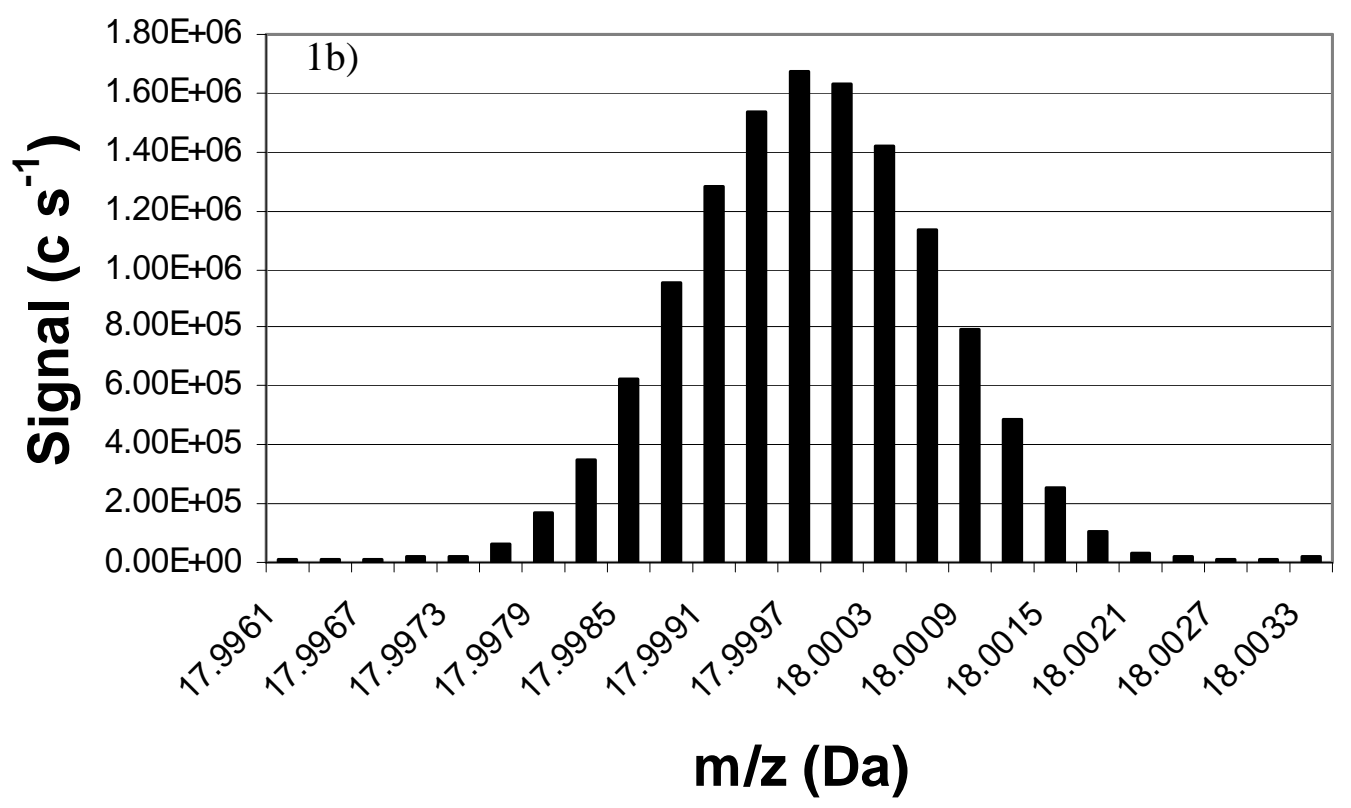

Fig. 1. (a) ${ }^{18} \mathrm{O}^{+}$spectrum acquired during solution nebulization ICP-MS (1\% nitric acid, $100 \mu \mathrm{L} \mathrm{min}{ }^{-1}$ nebulizer, Elemental Scientific Inc., Omaha NE) (b) ${ }^{18} \mathrm{O}^{+}$spectrum acquired during LA-ICP-MS (Y-doped glass) 


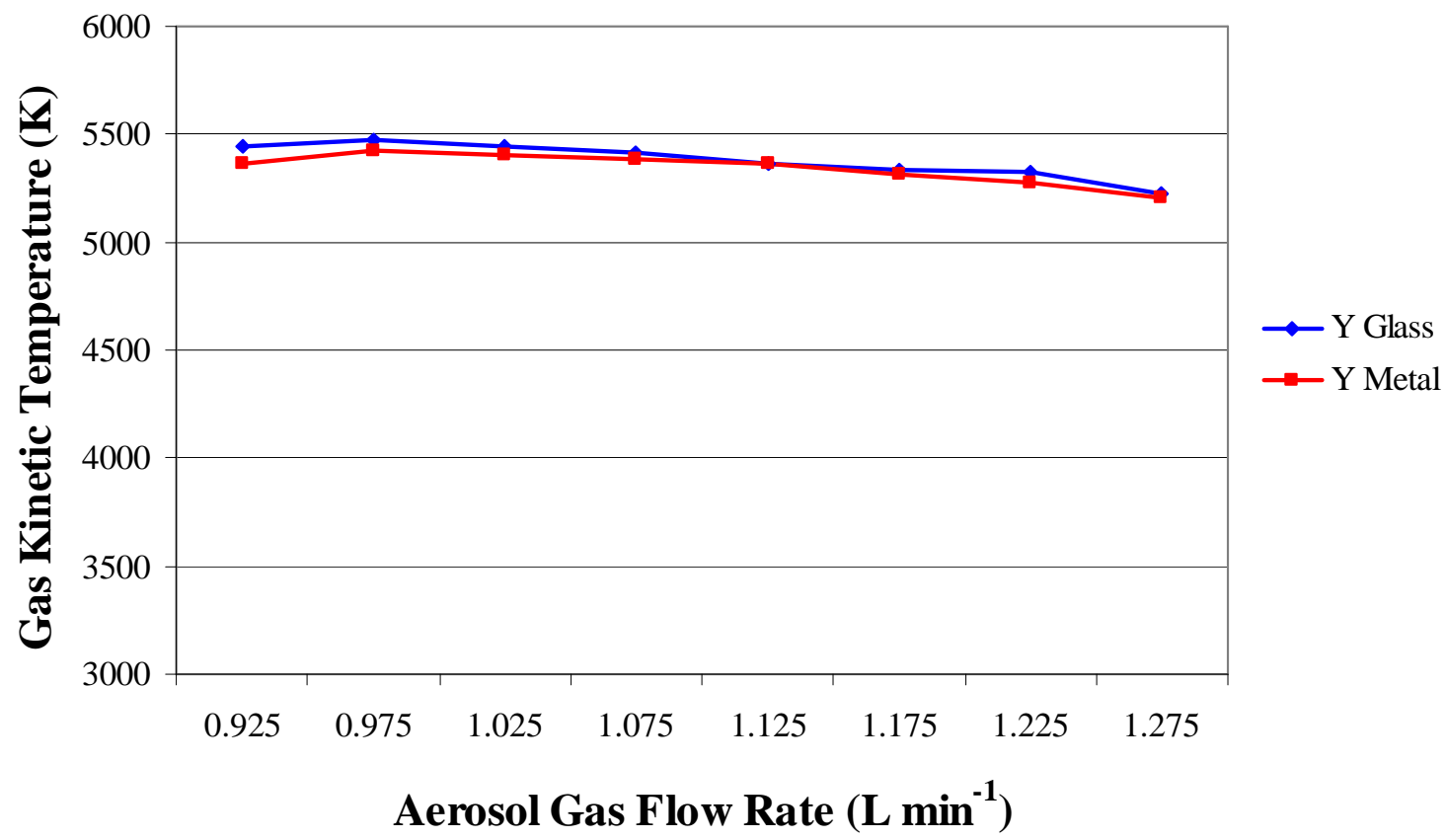

Fig. 2. Dependence of $\mathrm{T}_{\text {gas }}$ on argon aerosol flow rate for $\mathrm{YO}^{+} / \mathrm{Y}^{+}$for LA-ICP-MS analysis of Y glass and metal samples 


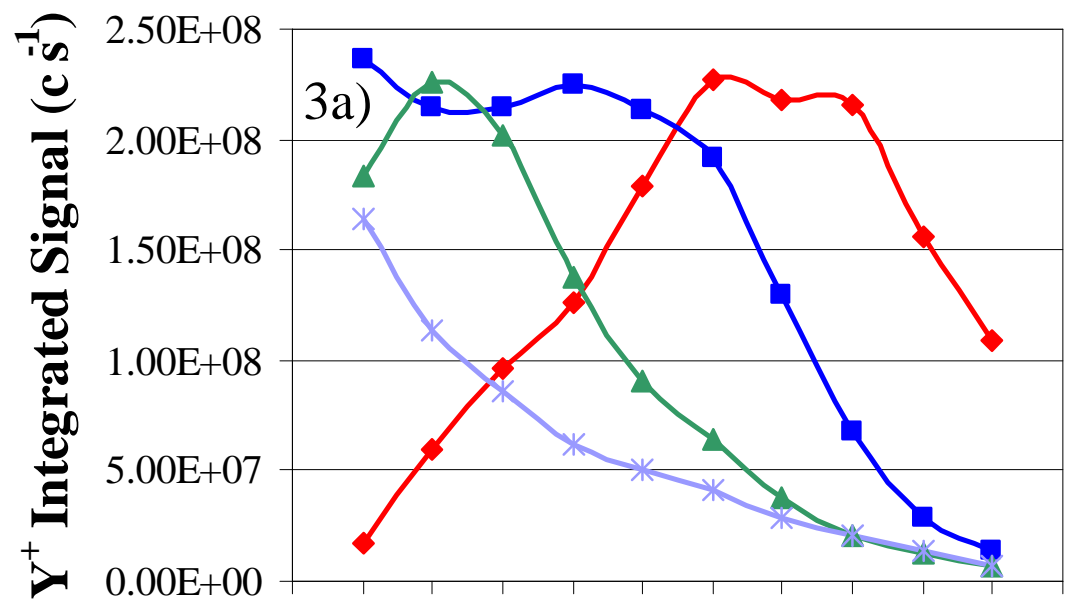

$\rightarrow 0 \mathrm{~mL} \mathrm{~N}_{2}$

$\rightarrow 5 \mathrm{~mL} \mathrm{~N}_{2}$

$\simeq 10 \mathrm{~mL} \mathrm{~N}_{2}$

* $20 \mathrm{~mL} \mathrm{~N}_{2}$

$\begin{array}{llllllllllll}0.3 & 0.4 & 0.5 & 0.6 & 0.7 & 0.8 & 0.9 & 1 & 1.1 & 1.2 & 1.3 & 1.4\end{array}$

Ar Make-up Gas Flow Rate (L min $^{-1}$ )

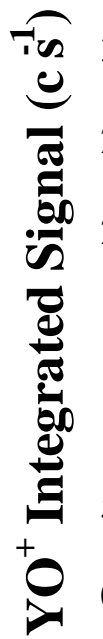

$3.00 \mathrm{E}+06$

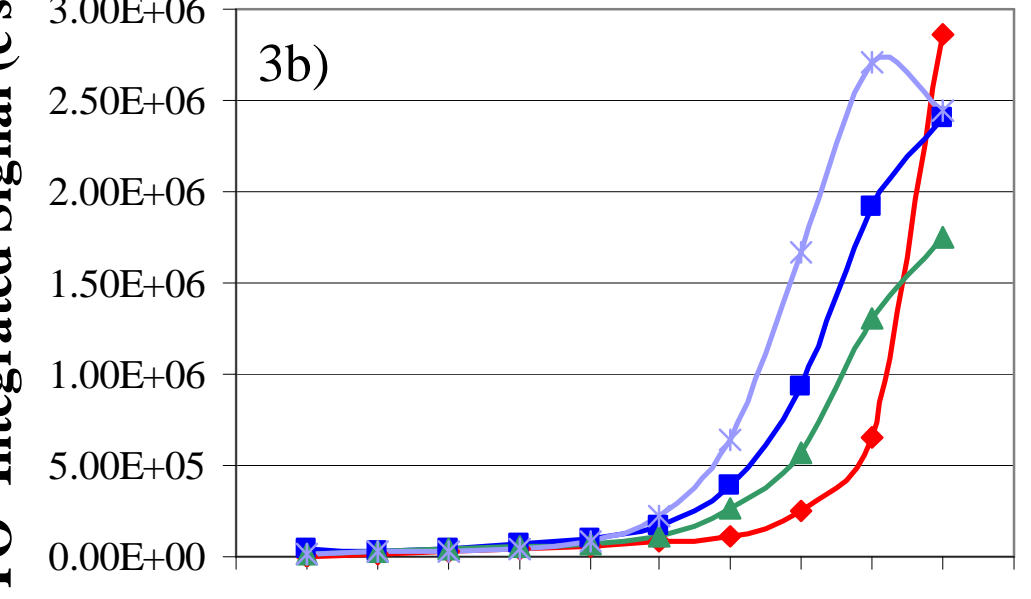

$\rightarrow 0 \mathrm{~mL} \mathrm{~N}_{2}$

$\rightarrow 5 \mathrm{~mL} \mathrm{~N}_{2}$

$-10 \mathrm{~mL} \mathrm{~N}_{2}$

* $20 \mathrm{~mL} \mathrm{~N}_{2}$

$\begin{array}{llllllllllll}0.3 & 0.4 & 0.5 & 0.6 & 0.7 & 0.8 & 0.9 & 1 & 1.1 & 1.2 & 1.3 & 1.4\end{array}$

Ar Make-up Gas Flow Rate (L min $^{-1}$ ) 


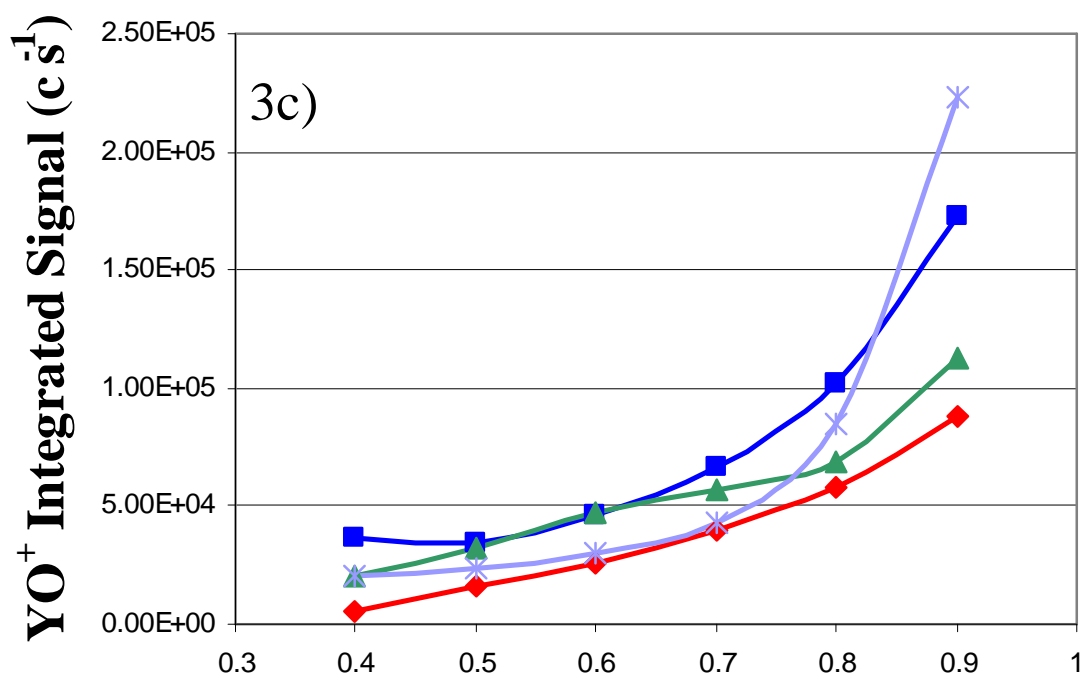

$\longrightarrow 0 \mathrm{~mL} \mathrm{~N}_{2}$

$\rightarrow-5 \mathrm{~mL} \mathrm{~N}_{2}$

$\rightarrow 10 \mathrm{~mL} \mathrm{~N}_{2}$

* $20 \mathrm{~mL} \mathrm{~N}_{2}$

Ar Make-up Gas Flow Rate (L min $\left.^{-1}\right)$

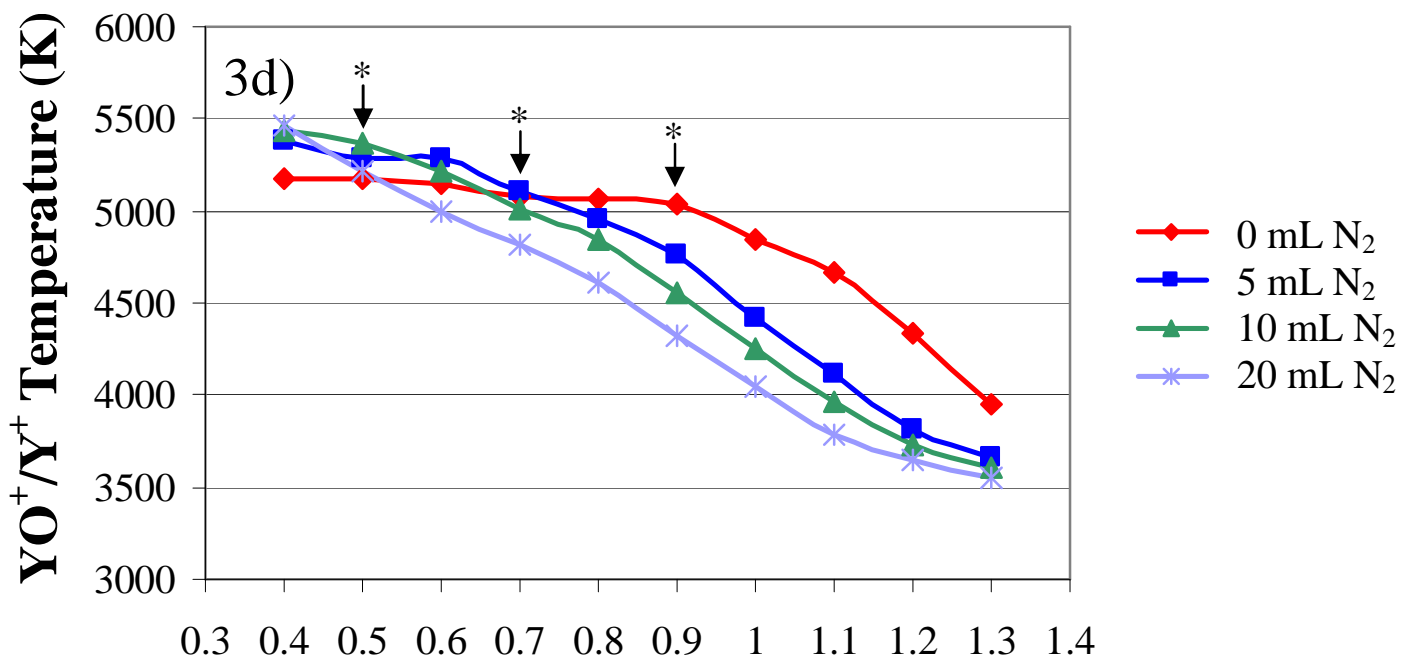

Ar Make-up Gas Flow Rate (L min $\left.^{-1}\right)$

Fig. 3. Effect of $\mathrm{N}_{2}$ addition on (a) $\mathrm{Y}^{+}$sensitivity (b) $\mathrm{YO}^{+}$sensitivity (c) $\mathrm{YO}+$ sensitivity at low Ar make-up gas (d) $\mathrm{T}_{\text {gas }}$ for $\mathrm{YO}^{+}$formation for $0.5 \mathrm{~L} \mathrm{~min}^{-1} \mathrm{Ar}$ aerosol gas plus Ar make-up gas

[* indicates flow rates corresponding to maximum $\mathrm{M}^{+}$signals at each $\mathrm{N}_{2}$ dose] 

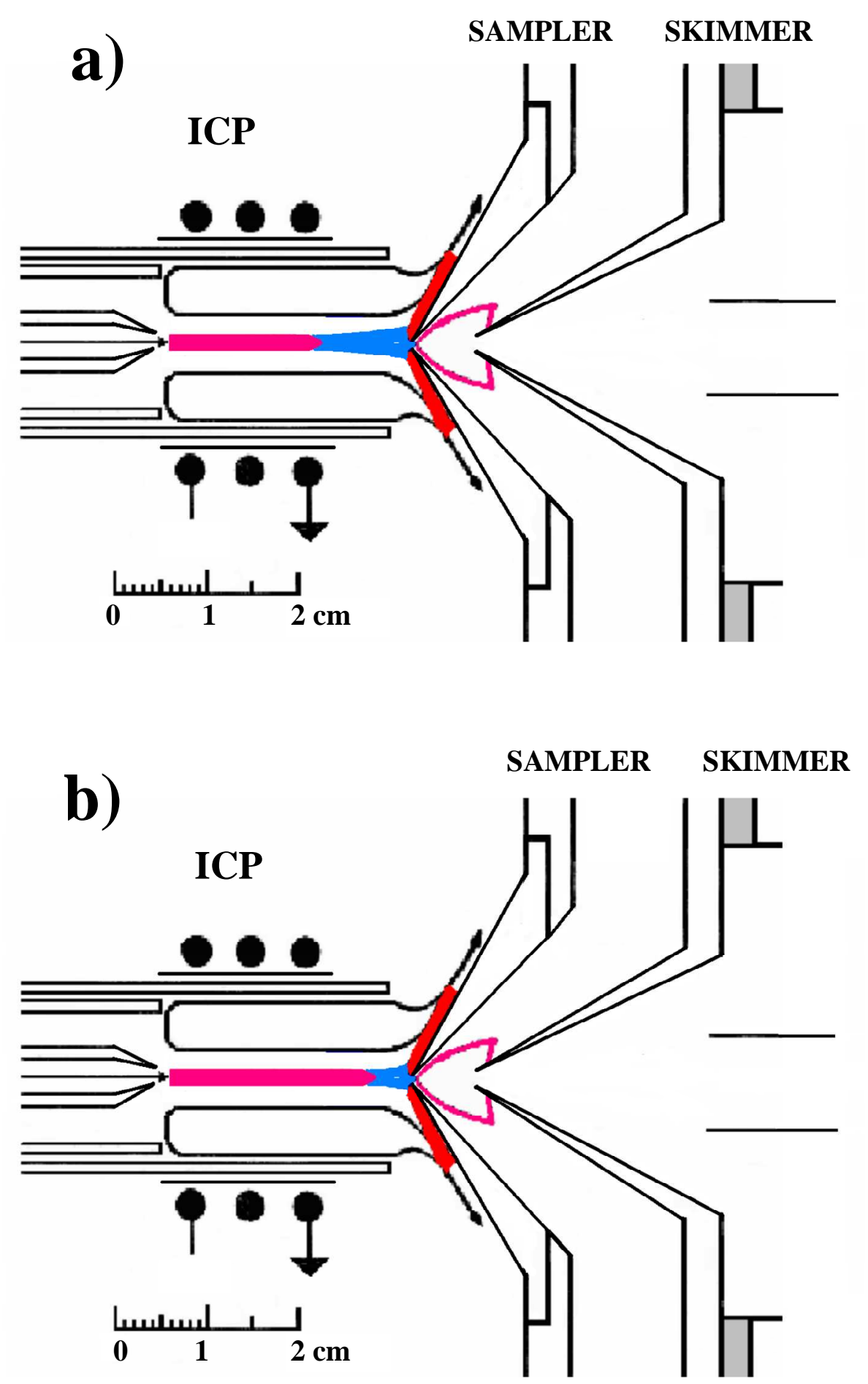

Fig. 4. Drawings of ICP, initial radiation zone (IRZ), and normal analytical zone (NAZ) during LA of $\mathrm{Y}_{2} \mathrm{O}_{3}$ pellet: a) at low aerosol gas flow rate, the red IRZ ends well upstream of the sampler; b) at higher aerosol gas flow rate, the IRZ tip moves closer to the sampler. (Adapted from Ref. 48.) 


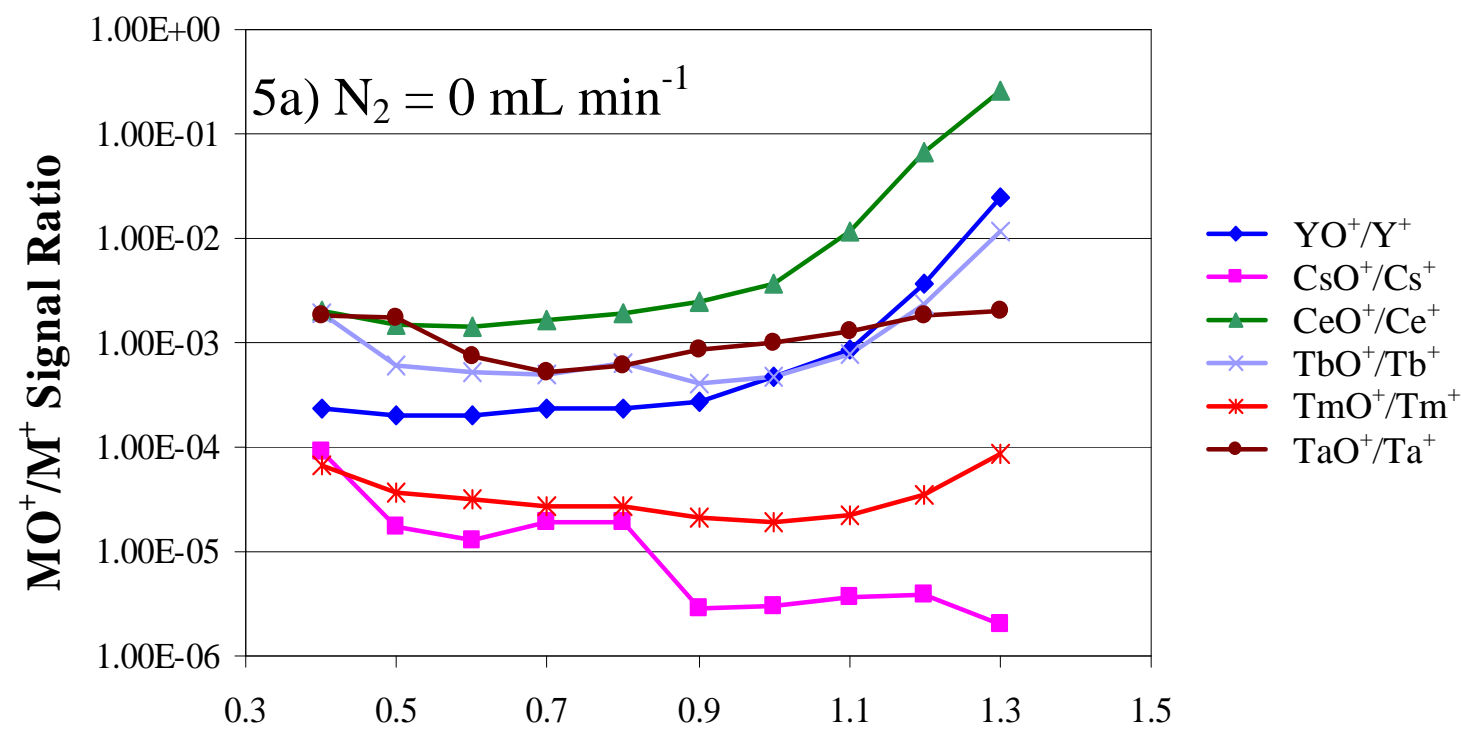

Ar Make-up Gas Flow Rate (L min $\left.^{-1}\right)$

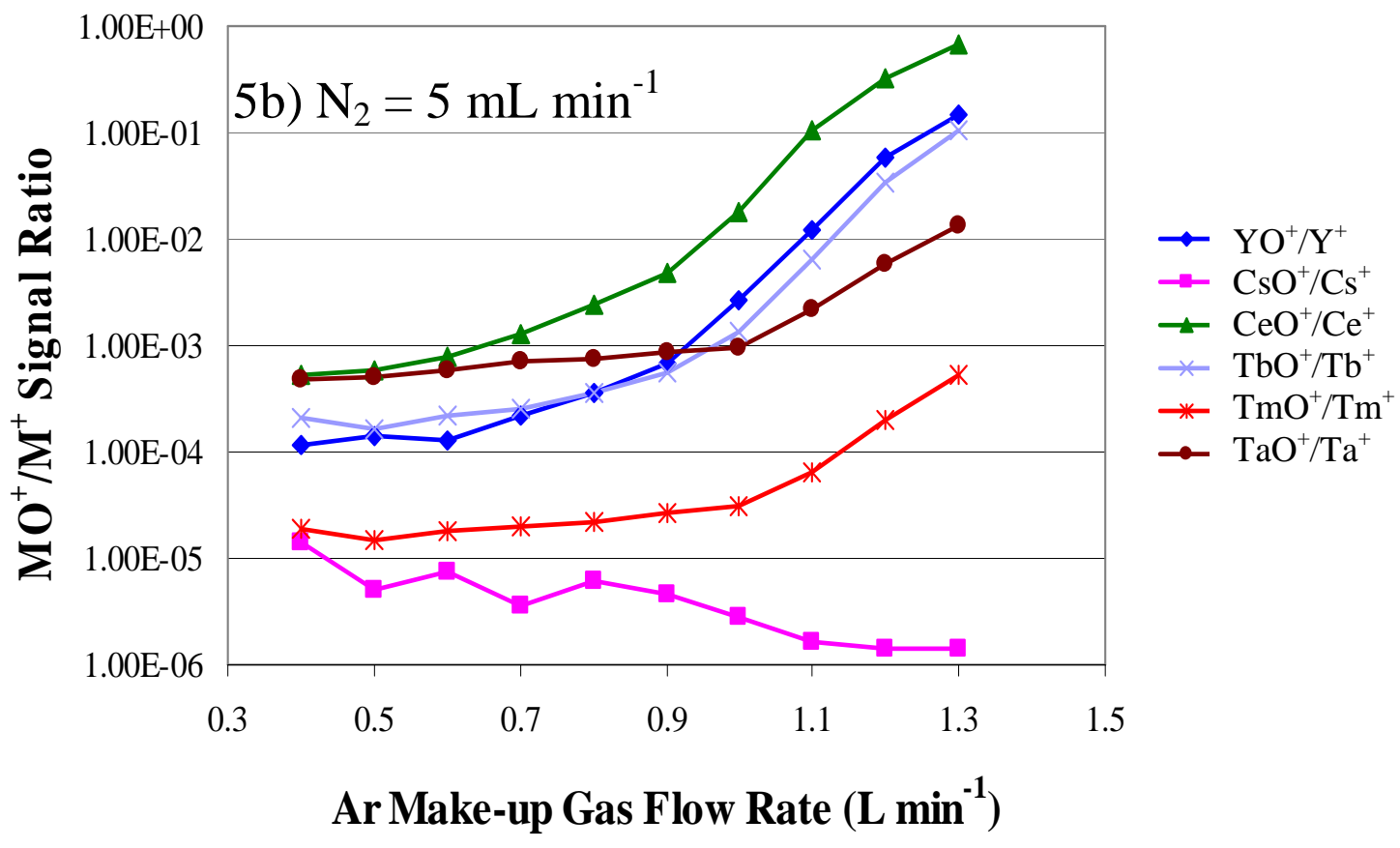




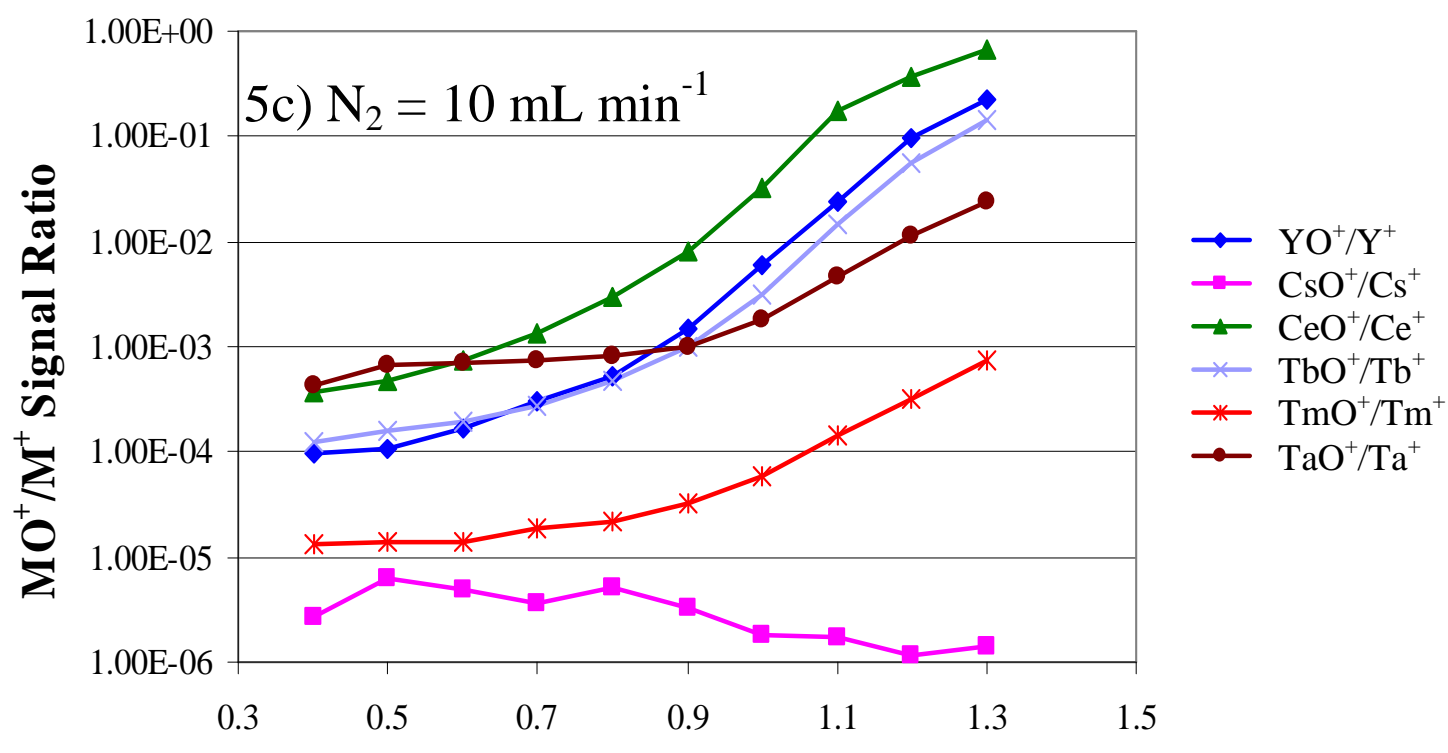

Ar Make-up Gas Flow Rate (L $\left.\min ^{-1}\right)$

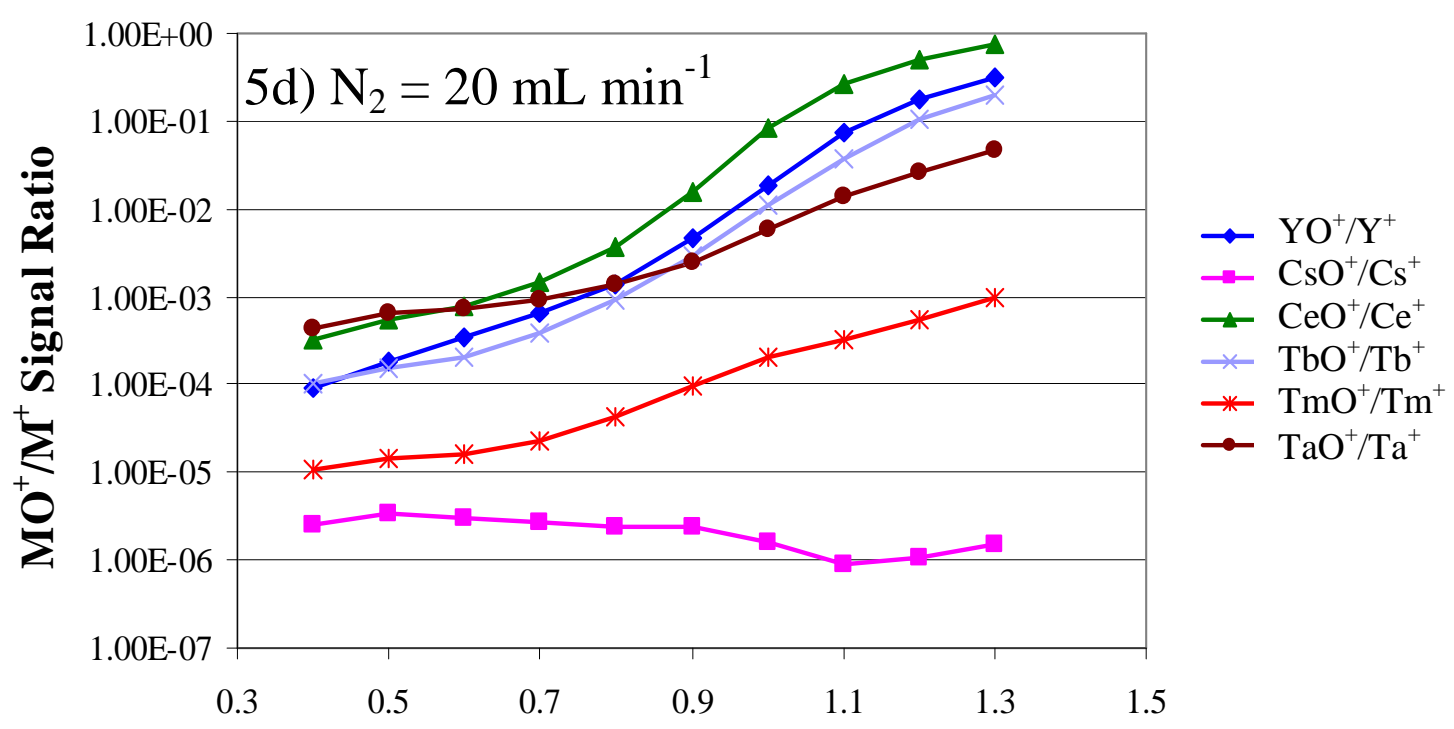

Ar Make-up Gas Flow Rate (L min $^{-1}$ )

Fig. 5. $\mathrm{MO}^{+} / \mathrm{M}^{+}$ratios in an Ar-only plasma with $\mathrm{N}_{2}$ additions to the central channel of a) $0 \mathrm{~mL} \mathrm{~min}^{-1}$ b) $5 \mathrm{~mL} \mathrm{~min}{ }^{-1}$ c) $10 \mathrm{~mL} \mathrm{~min}{ }^{-1}$ d) $20 \mathrm{~mL} \mathrm{~min}^{-1}$ 


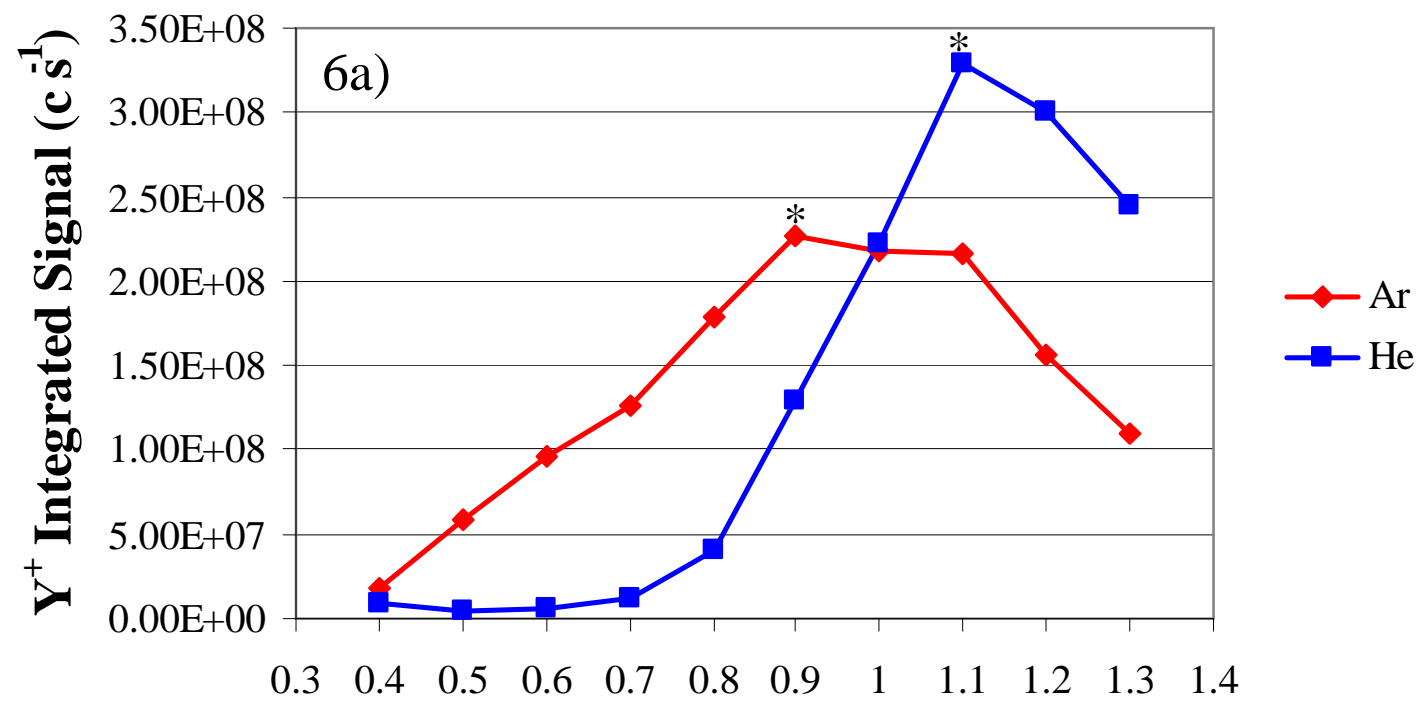

Ar Make-up Gas Flow Rate (L min-1)

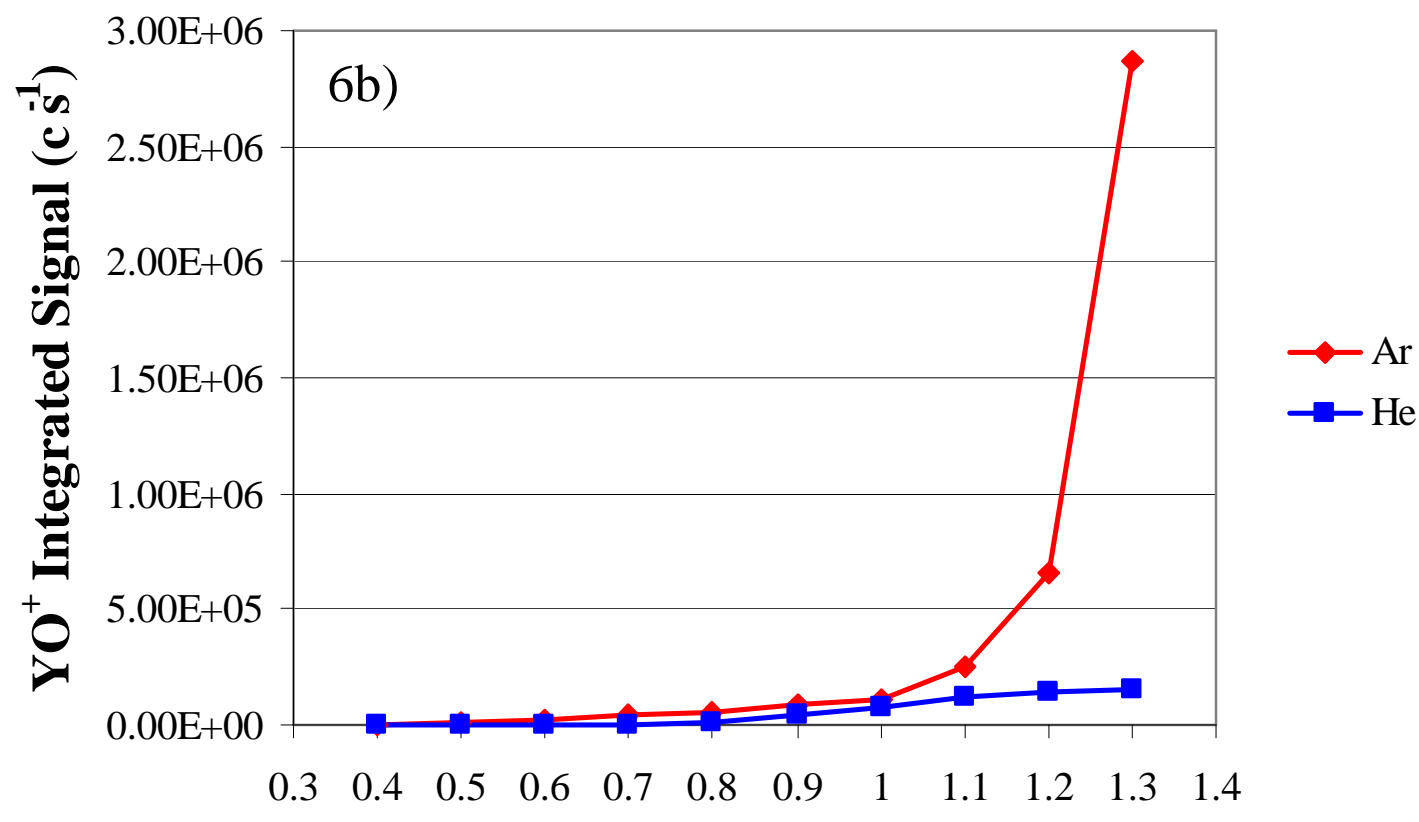

Ar Make-up Gas Flow Rate (L min-1) 


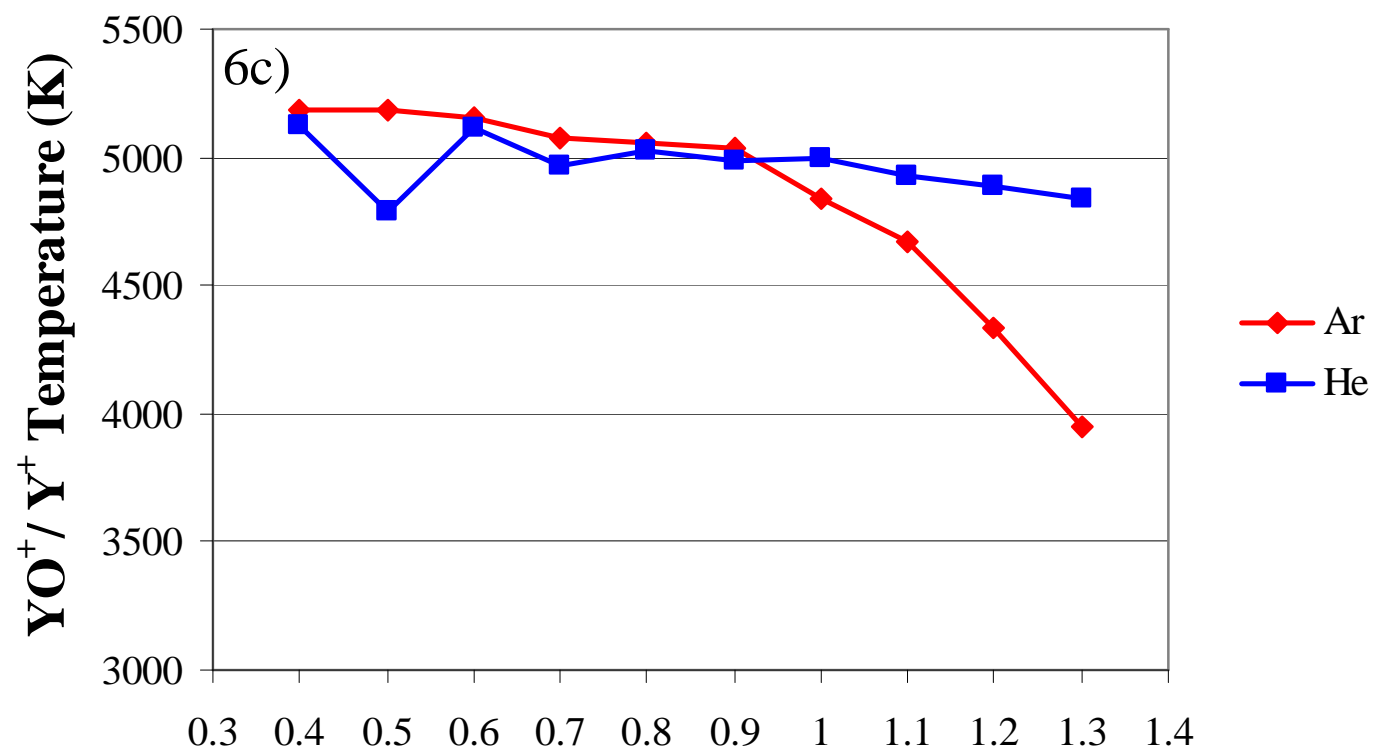

Ar Make-up Gas Flow Rate $\left(L \mathrm{~min}^{-1}\right)$

Fig. 6. Effect of He addition $\left(0.5 \mathrm{~L} \mathrm{~min}^{-1}\right)$ on (a) $\mathrm{Y}^{+}$sensitivity [* denotes optimum gas flow for maximum $\mathrm{Y}^{+}$signal] (b) $\mathrm{YO}^{+}$sensitivity (c) $\mathrm{T}_{\text {gas }}$ for $\mathrm{YO}^{+}$formation for $0.5 \mathrm{~L} \mathrm{~min}^{-1} \mathrm{He}$ aerosol gas plus Ar make-up gas 


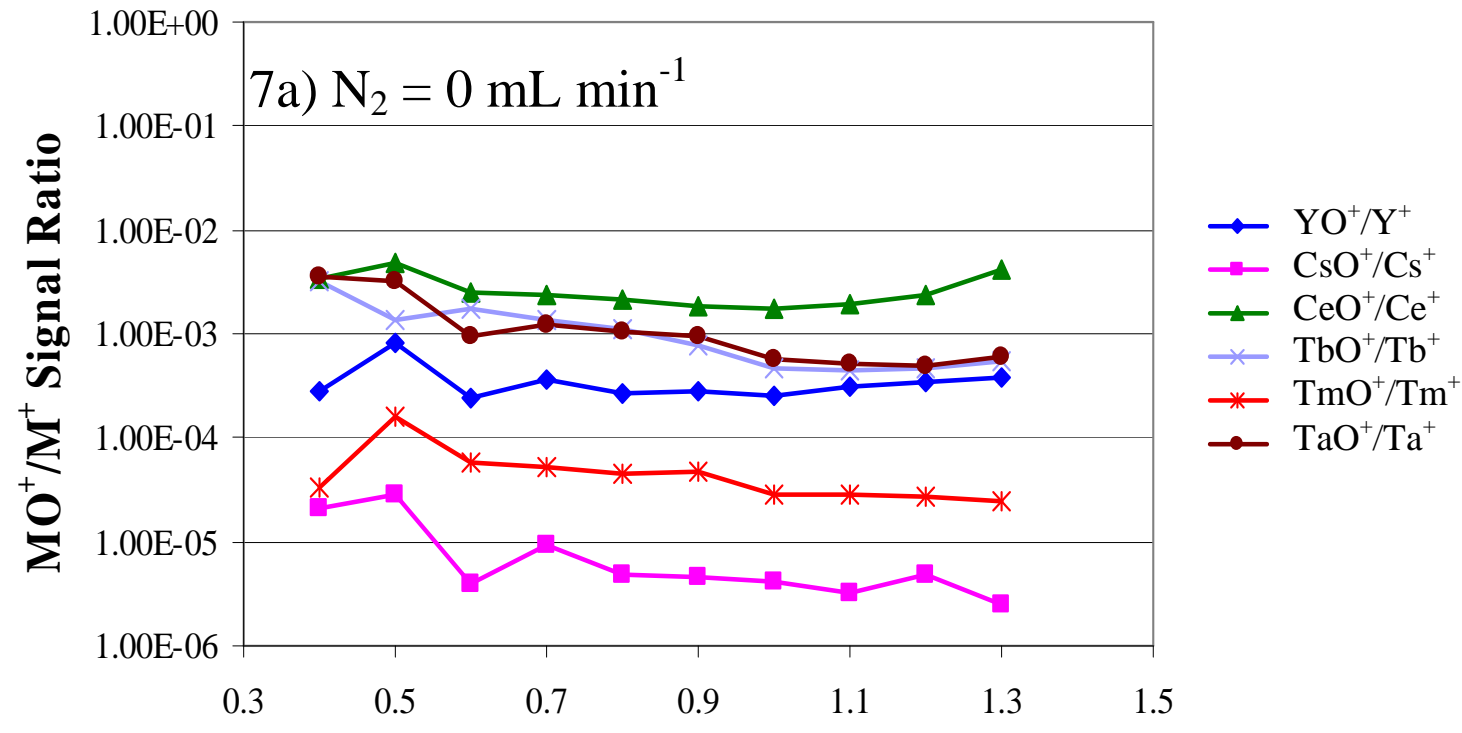

Ar Make-up Gas Flow Rate (L min $\left.{ }^{-1}\right)$

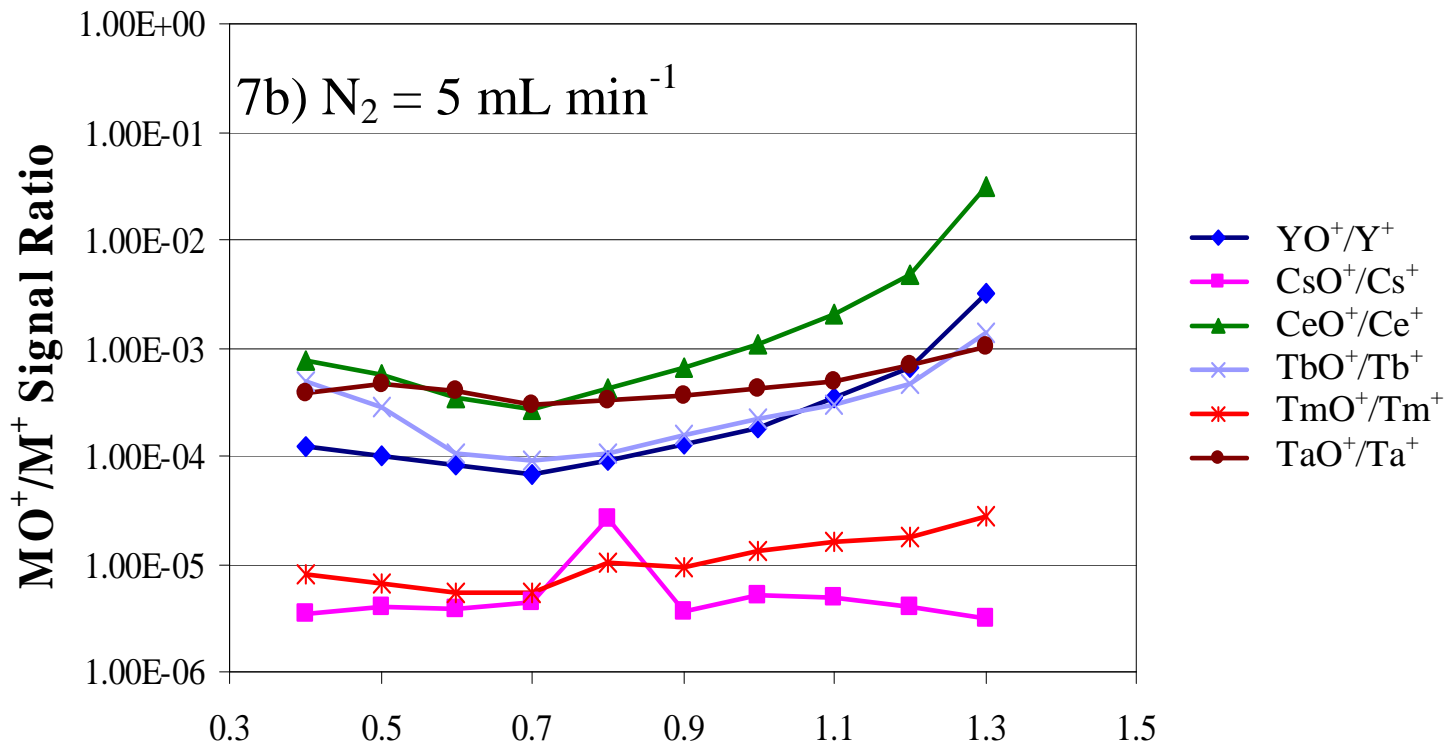

Ar Make-up Gas Flow Rate (L min ${ }^{-1}$ ) 


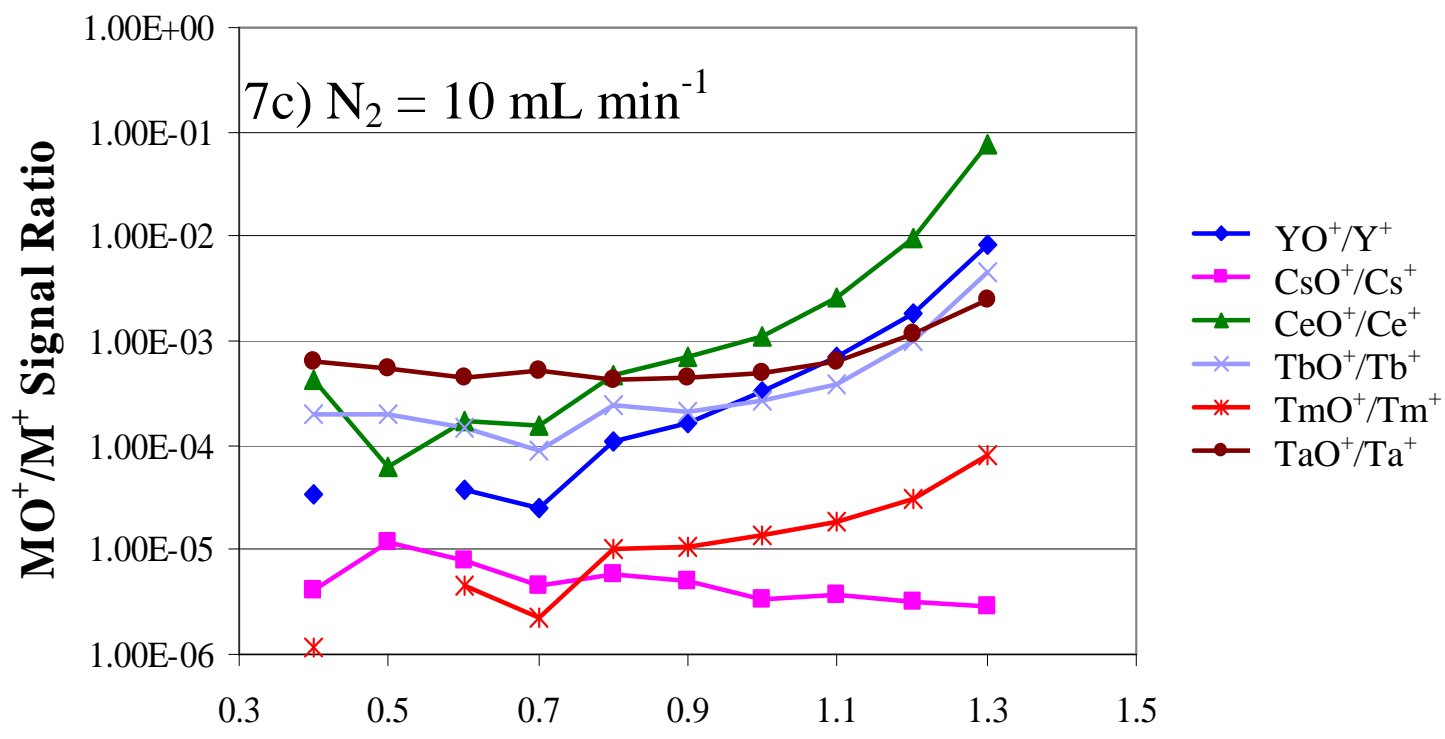

Ar Make-up Gas Flow Rate $\left(\mathrm{L} \mathrm{min}^{-1}\right)$

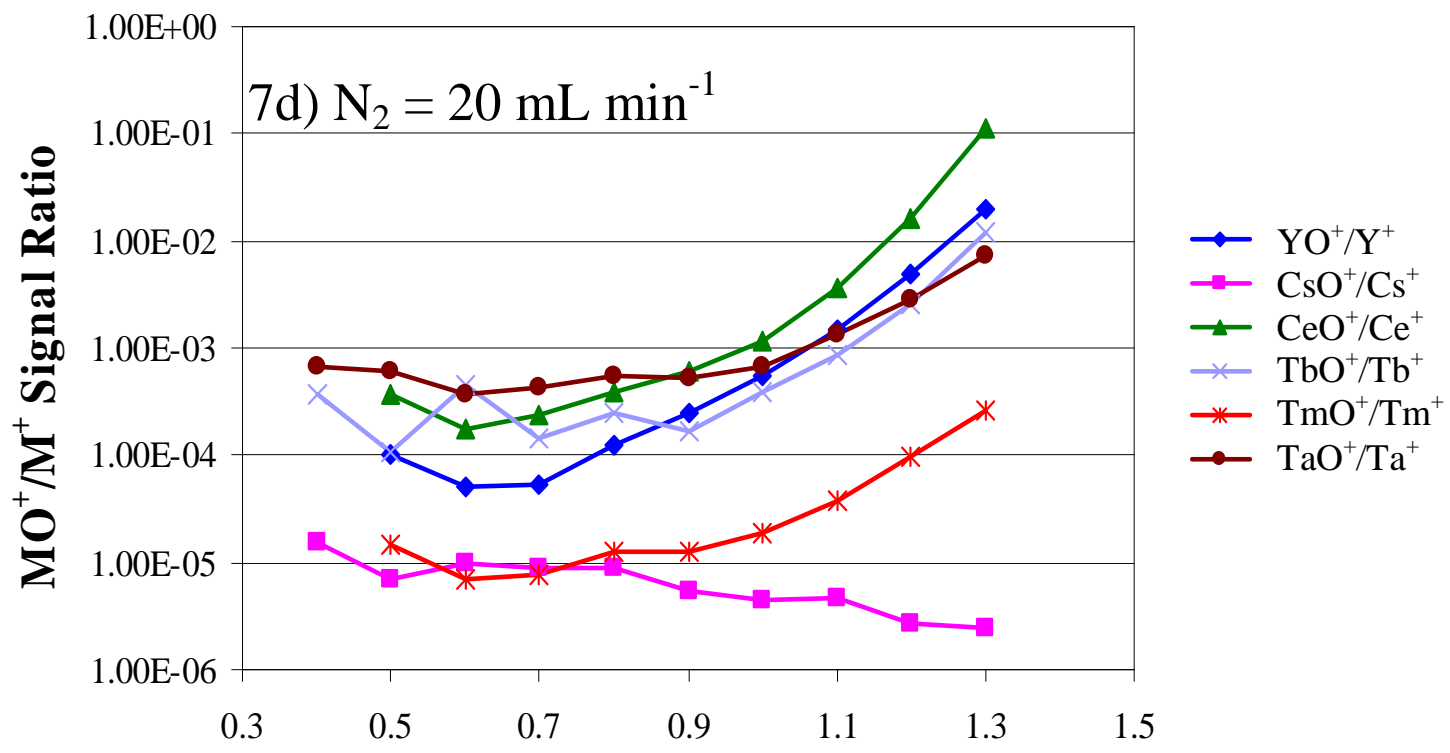

Ar Make-up Gas Flow Rate $\left(\mathrm{L} \mathrm{min}^{-1}\right)$

Fig. 7. $\mathrm{MO}^{+} / \mathrm{M}^{+}$ratios in a $\mathrm{He}\left(0.5 \mathrm{~L} \mathrm{~min}^{-1}\right)$-Ar plasma with $\mathrm{N}_{2}$ addition to the central channel of a) $0 \mathrm{~mL} \mathrm{~min}^{-1}$ b) $5 \mathrm{~mL} \mathrm{~min}{ }^{-1}$ c) $10 \mathrm{~mL} \mathrm{~min}^{-1}$ d) $20 \mathrm{~mL} \mathrm{~min}^{-1}$ 


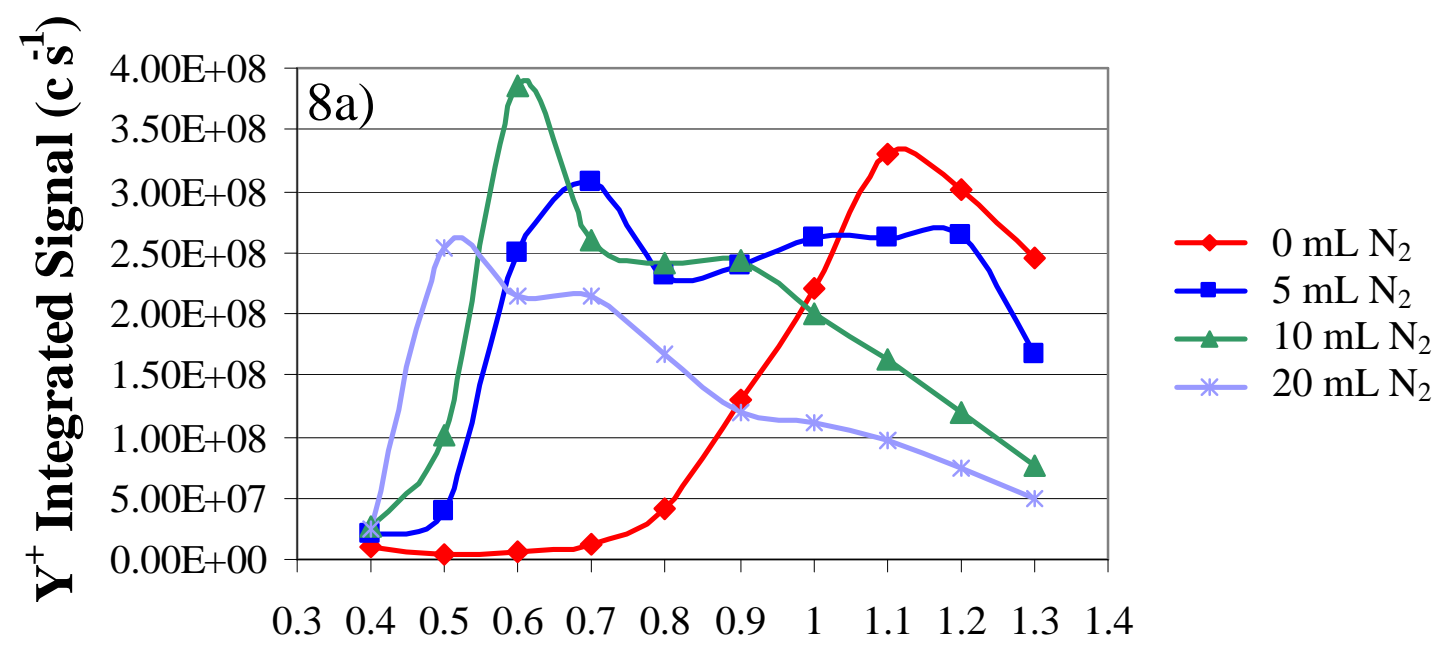

Ar Make-up Gas Flow Rate (L min ${ }^{-1}$ )

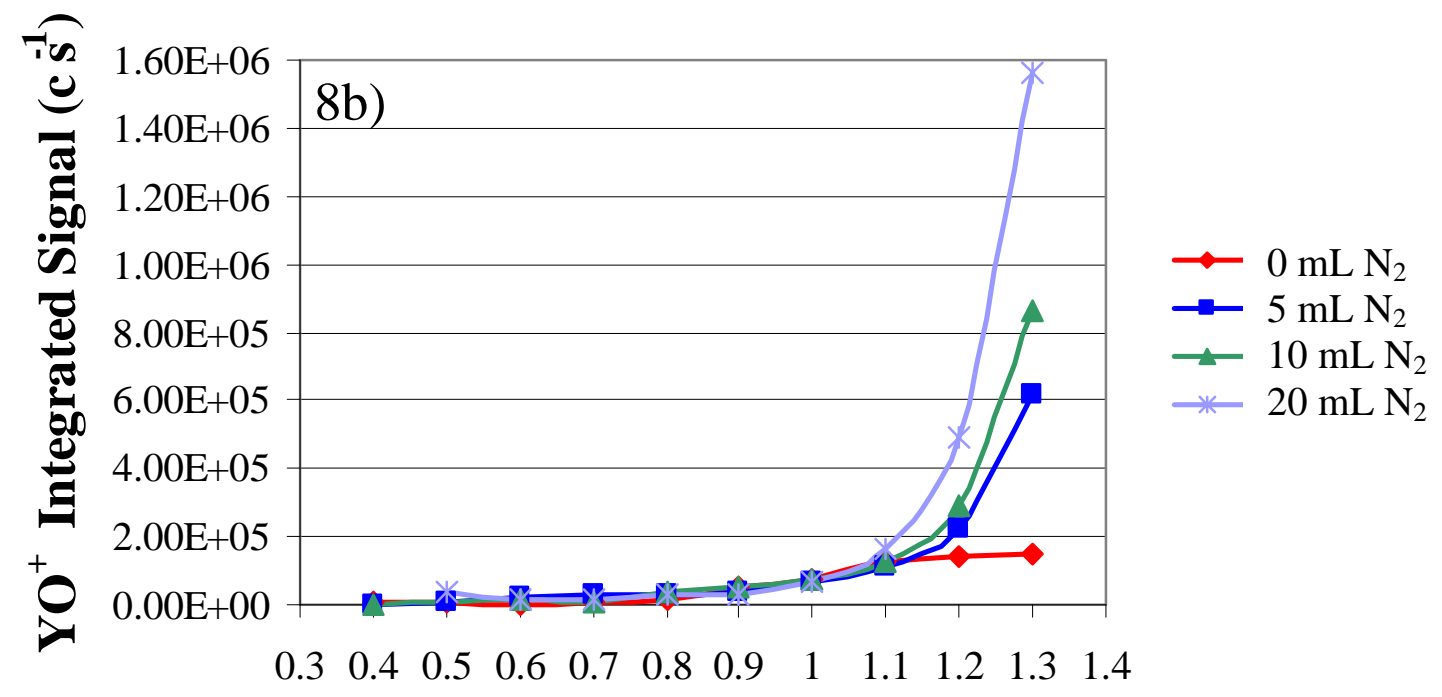

Ar Make-up Gas Flow Rate $\left(\mathrm{L} \mathrm{min}^{-1}\right)$ 


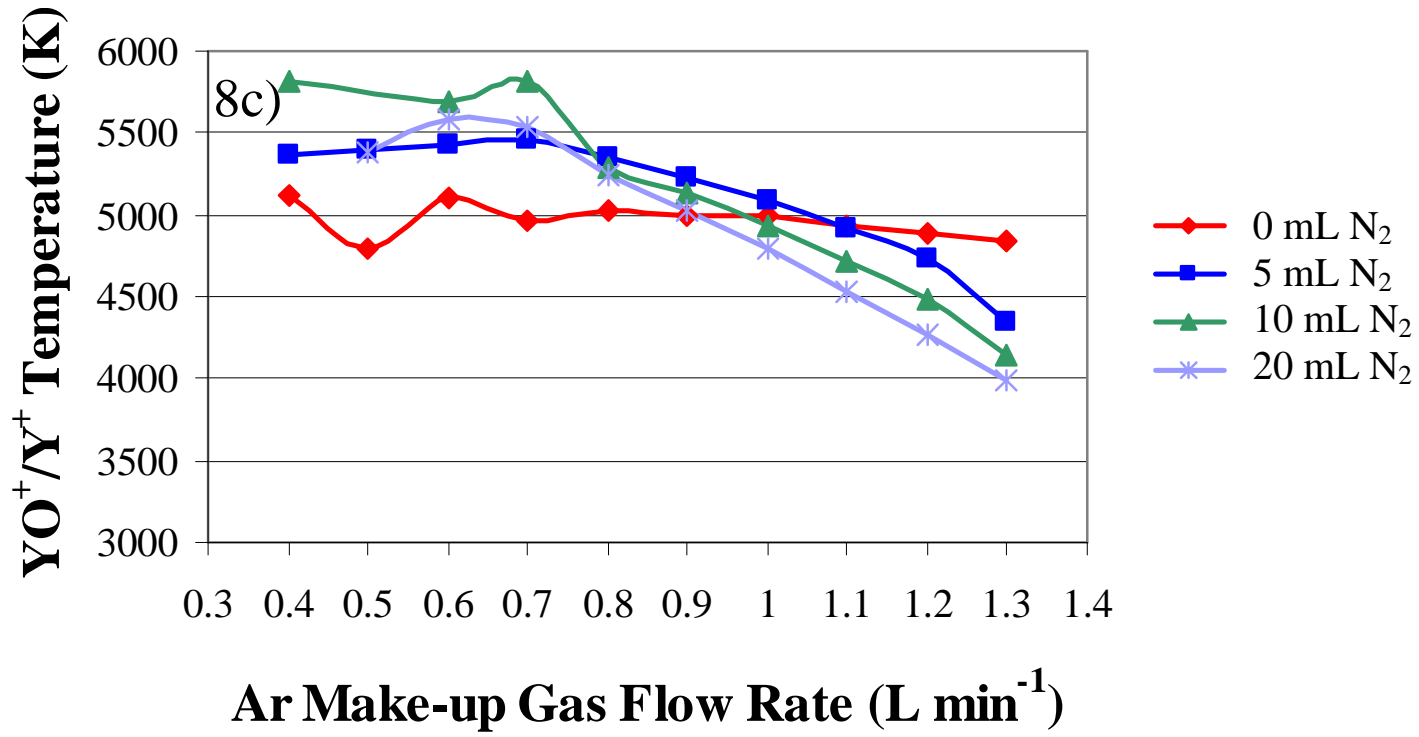

Fig. 8. Effect of $\mathrm{N}_{2}$ addition on (a) $\mathrm{Y}^{+}$sensitivity (b) $\mathrm{YO}^{+}$sensitivity (c) $\mathrm{T}_{\text {gas }}$ for $\mathrm{YO}^{+}$ formation for He aerosol gas $\left(0.5 \mathrm{~L} \mathrm{~min}^{-1}\right)$ plus Ar make-up gas 


\title{
Chapter 3. Examination of the Origins of Metal Argide Ions in Laser Ablation-Inductively Coupled Plasma-Mass Spectrometry
}

\author{
Travis Witte and R. S. Houk
}

\begin{abstract}
The abundance of metal argide (MAr $\left.{ }^{+}\right)$ions during laser ablation- inductively coupled plasma-mass spectrometry (LA-ICP-MS) is measured during ablation of pure samples of transition metals. The relative abundance of $\mathrm{MAr}^{+}$ions to $\mathrm{M}^{+}$ions for each element increases as the dissociation energy $\left(D_{0}\right)$ of the ions increases. Gas kinetic temperatures $\left(\mathrm{T}_{\mathrm{gas}}\right)$ are determined from the calculated $\mathrm{MAr}^{+} / \mathrm{M}^{+}$ratios and are used to indicate the origins of $\mathrm{MAr}^{+}$ions. Based on the determined $\mathrm{T}_{\text {gas }}$ values, $\mathrm{MAr}^{+}$ions are found to be much less abundant in the mass spectrum than expected based upon plasma conditions. Collision-induced dissociation during the ion extraction process is suggested to be responsible for removal of $\mathrm{MAr}^{+}$ions. Factors responsible for these collisions are discussed.
\end{abstract}

\section{Introduction}

Inductively coupled plasma mass spectrometry (ICP-MS) is one of the most sensitive techniques for atomic analysis, owing to the efficiency and robustness of the ICP as an atomic ion source [1,2]. The gas composition of the plasma is a significant factor in determining the ionization efficiency of the ICP. While a number of gases have been utilized to generate the ICP, including nitrogen [3,4], oxygen [5], helium [6,7], neon [8], and air 
$[3,9]$, argon remains the gas of choice for plasma generation in ICP-MS. Argon offers several advantages for ICP operation. Compared to some alternative gases, the low cost of Ar better suits it for the high gas consumption needed for long-term operation of the plasma. The ionization energy of $\operatorname{Ar}(15.76 \mathrm{eV})$ and the excitation energy of the first excited state $(11.55 \mathrm{eV})$ are sufficiently high to achieve greater than $90 \%$ ionization of most elements but low enough that formation of doubly-charged analyte ions is minor [10]. As an inert gas $\mathrm{Ar}$ is chemically unreactive with analyte species in the plasma, which minimizes the formation of unwanted molecular ions during atomic analysis.

Molecular Ar species are not absent entirely, however. Given the high number density of Ar in the ICP, if even a small percentage of Ar atoms form molecular ions with other species in the plasma, the resulting Ar-based polyatomic ion interferences can significantly impact the mass spectrum. Diatomic ions, such as $\mathrm{Ar}_{2}{ }^{+}, \mathrm{ArH}^{+}$and $\mathrm{ArO}^{+}$, which incorporate major background species in the plasma are the most abundant of these interferences [1]. These ions are endemic to all ICP-MS analyses, particularly those in which the sample is introduced as a solution. Accordingly, studies have been undertaken of this variety of Ar polyatomic ion to better understand the fundamental processes in the ICP related to their abundance in the mass spectrum [11-13].

Interferences arising from the formation of Ar adducts with analyte ions in the plasma are less prominent but may still lead to greater complications in the mass spectrum. Unlike the polyatomic ions formed by the combination of Ar and background species, where only the same handful of masses must be monitored for interferences, an Ar-based interference is possible at every mass equal to that of the analyte and the major isotope of Ar combined. In certain $\mathrm{m} / \mathrm{z}$ regions these $\mathrm{MAr}^{+}$ions cannot be readily resolved from so-called "isobaric" 
atomic analyte ions with high-resolution magnetic sector instruments. Foreknowledge of sample composition and chemical separations can assist in surmounting this obstacle but may complicate the analysis or be impracticable in some situations.

One such situation is encountered for solid samples where spatial integrity needs to be preserved, whether to gain additional information during the analysis or to minimize the destruction of a valuable sample. Laser ablation (LA)-ICP-MS can readily analyze such samples, with the concession that all species present in the sample, including those that may form polyatomic interferences, will be introduced into the ICP-MS. Guillong et al. describe a specific case where these $\mathrm{MAr}^{+}$ions complicate analysis, the determination of Platinum Group Elements (PGEs) in ore samples [14]. The base metals that occur alongside PGEs in these samples can form metal argide ions $\left(\mathrm{MAr}^{+}\right)$that occur at almost the same $\mathrm{m} / \mathrm{z}$ values as the $\mathrm{M}^{+}$ions from the low-abundance PGEs.

Studies to better understand and address $\mathrm{MAr}^{+}$ions in ICP-MS analyses, for either solution nebulization or LA, have only been undertaken by a few investigators $[15,16]$. Becker et al. compared the abundance of $\mathrm{MAr}^{+}$ions in solution nebulization ICP-MS, LAICP-MS and glow discharge (GD)-MS and investigated the periodic nature of their abundances [17]. Guillong et al. described the effect of the mass spectrometer interface design in commercial quadrupole instruments on $\mathrm{MAr}^{+}$ion abundance [14].

The present work seeks to further build upon the knowledge about $\mathrm{MAr}^{+}$ions presented in these investigations. By comparing theoretical calculations of the $\mathrm{MAr}^{+} / \mathrm{M}^{+}$ion ratio at various conditions with a $\mathrm{MAr}^{+} / \mathrm{M}^{+}$ratio measured from empirical ion signals, the conditions responsible for the observed abundance of $\mathrm{MAr}^{+}$ions can be determined. 
Potentially, information on the origin of these ions could be utilized to develop broad strategies needed for their reduction.

\section{Theory}

\section{Determination of Polyatomic Ion Origin}

Prior investigations have developed a general method to describe the origins of polyatomic ions in ICP-MS $[11,13,18,19]$. The basis of this method is a proposed dissociation reaction for the polyatomic species, in this case $\mathrm{MAr}^{+}$.

$$
\mathrm{MAr}^{+} \rightleftharpoons \mathrm{M}^{+}+\operatorname{Ar} \Delta \mathrm{H}=\mathrm{D}_{0}\left(\mathrm{MAr}^{+}\right)
$$

Experimental data are collected and used to evaluate a dissociation expression for the proposed reaction.

$$
K_{d}=\left(\frac{n_{M^{+}}}{n_{M A r^{+}}}\right) n_{A r}
$$

In this expression, the ratio between the number densities $(n)$ for $\mathrm{M}^{+}$and $\mathrm{MAr}^{+}$is assumed to be proportional to the ratio between the measured signals of the ions $\mathrm{M}^{+}$and $\mathrm{MAr}^{+}$. Use of this measured signal ratio removes the need to ascertain the absolute number densities of each ion. An estimate of the number density of the neutral species $\left(n_{\mathrm{Ar}}\right)$ is made from known plasma conditions.

An alternative form of the same dissociation expression can be written in relation to the partition functions $(\mathrm{Z})$ of each species, using the available spectroscopic constants (Table 1) $[20-31]$.

$$
K_{d}=\frac{Z_{A r} Z_{M^{+}}}{Z_{M A r^{+}}} e^{-D_{0} / k T}
$$


In the expanded form of this expression (not shown), the electronic partition function is assumed to be equal to the statistical weight of the ground state $(\mathrm{g})$ of the ion $\mathrm{MAr}^{+}$. When the term symbol of the ground state is sigma, $g=$ the multiplicity of ion. For all other term symbols, $g=2 *$ (multiplicity of the ground electronic state of $\mathrm{MAr}^{+}$) [32,33]. Calculation of the electronic partition function for $\mathrm{M}^{+}$utilizes a polynomial equation valid from 1500 to $12000 \mathrm{~K}$ for each element $[34,35]$. Use of these equations at greater temperatures represents the best approximation available and is necessary for the very high $\mathrm{T}_{\text {gas }}$ values encountered in this study. Both the neutral Ar number density and the partition functions depend on the gas kinetic temperature ( $\mathrm{T}_{\text {gas }}$ ) and thus are calculated across a range of $\mathrm{T}_{\text {gas }}$ values. In this study, the range of $\mathrm{T}_{\text {gas }}$ values extends beyond the limit cited for the atomic partition functions.

A $\mathrm{T}_{\text {gas }}$ value is determined for the reaction by comparing the measured dissociation constant $\left(\mathrm{K}_{\mathrm{d}}\right)$ from Eq. 2 and theoretical dissociation constant from Eq. 3 across the likely range of reasonable $T_{\text {gas }}$ values. The value at which the measured and theoretical $K_{d}$ values best agree is assigned as the $T_{\text {gas }}$ representative of the conditions responsible for the observed abundance of polyatomic ions in the spectrum.

Based on these $T_{\text {gas }}$ values, there are three possible assignments that can be made for the origin of the polyatomic ion. (1) At $\mathrm{T}_{\text {gas }}$ values between $\sim 5000$ to $6000 \mathrm{~K}$, the polyatomic ion abundance in the spectrum is consistent with that expected in the plasma. Post-plasma effects do not alter the polyatomic ion abundance. (2) At $\mathrm{T}_{\text {gas }}$ values greater than $6000 \mathrm{~K}$, some process(es) during ion extraction removes the polyatomic ion, leaving it less abundant in the spectrum than expected based on plasma conditions alone. (3) At $\mathrm{T}_{\text {gas }}$ values below $5000 \mathrm{~K}$, some process during extraction creates additional polyatomic ions, causing it to be more abundant in the spectrum than expected from plasma conditions. 


\section{Experimental}

\section{Instrumentation}

LA-ICP-MS experiments were conducted using an LSX-500 (CETAC Technologies, Inc., Omaha, NE) connected to an XSeries 2 (Thermo Fisher Scientific, Inc., Bremen, Germany). The LSX-500 is a $266 \mathrm{~nm} \mathrm{Nd:YAG} \mathrm{system} \mathrm{with} \mathrm{a} \mathrm{laser} \mathrm{pulse} \mathrm{width} \mathrm{of} 5 \mathrm{~ns}$ and maximum energy output of $9 \mathrm{~mJ}$. Beam characteristics, including laser energy, repetition rate and spot size are selected through a software interface. The XSeries 2 ICP-MS was operated with a grounded guard electrode to minimize the secondary discharge and the kinetic energy spread of the ions. This device is a quadrupole, unlike the magnetic sector instrument utilized in a previous paper to investigate $\mathrm{MO}^{+}$ions during LA [36]. A collision cell is available in this instrument but was operated with no added collision gas. This ensured that polyatomic ion abundances were influenced solely by conditions in the plasma and extraction interface.

All gas flows were Ar. Most gas flows were regulated with the internal mass flow controllers of the XSeries 2. An external Ar mass flow controller was used to control the aerosol gas flow for laser ablation. The instrument was tuned using atomic ions from nebulized solutions to maximize signal and while maintaining reasonable stability. The torch inlet was then connected to the laser system for final adjustment before data collection. Settings for both the LSX-500 and XSeries 2 are summarized in Table 2.

\section{Materials}

Single metal samples (Materials Preparation Center, Ames Laboratory, U.S. Department of Energy, Ames, IA) were obtained for each of nine first-row transition metals 
(Ti, V, Cr, Mn, Fe, Co, Ni, Cu, Zn). Since the $\mathrm{MAr}^{+}$ions occur at low abundance, $\mathrm{M}^{+}$ions from impurity elements could be mistaken for the polyatomic. Various isotopes corresponding to heavy metal impurities were monitored, which confirmed that only $\mathrm{MAr}^{+}$ ions were detected at the masses of interest, not $\mathrm{M}^{+}$ions from elemental impurities. NIST 1263a Cr-V Modified Steel was ablated to collect multielement data for construction of a mass bias plot.

\section{Estimation of Neutral Density $\left(n_{\mathrm{Ar}}\right)$}

It is necessary to estimate the number density of the neutral species in the proposed dissociation reaction to calculate the measured dissociation constant. For the $\mathrm{MAr}^{+}$ions examined in this study, that requires estimation of the number density of neutral $\operatorname{Ar}\left(n_{\mathrm{Ar}}\right)$. The number density of the major components in the plasma can be estimated using the ideal gas law.

$$
n_{\text {total }}=n_{\mathrm{Ar}}+n_{\mathrm{H}}+n_{\mathrm{O}}=\mathrm{P} / \mathrm{RT}_{\text {gas }}
$$

In LA-ICP-MS experiments, $n_{\mathrm{H}}$ and $n_{\mathrm{O}}\left(\sim 4 \times 10^{15}\right.$ and $2 \times 10^{15} \mathrm{~cm}^{-3}$, respectively $)$ are assumed to be negligible relative to $n_{\mathrm{Ar}}\left(\sim 1 \times 10^{18} \mathrm{~cm}^{-3}\right.$ at $\mathrm{P}=1 \mathrm{~atm}$ and $\left.\mathrm{T}_{\text {gas }} \sim 6000 \mathrm{~K}\right)$, especially when ablating metals.

\section{Mass Bias Corrections}

The preferential transmission and detection of heavier ions relative to lighter ions, known as mass bias, is an issue endemic to ICP-MS analysis. Corrections for this behavior are necessary for analyses involving the measurement of accurate isotope ratios, especially when the mass difference between isotopes is large. $\mathrm{The} \mathrm{MAr}^{+}$ions in this study present an 
extreme case, with $\mathrm{M}^{+}$ions $40 \mathrm{Da}$ lighter than their corresponding $\mathrm{MAr}^{+}$ion. The effect of these corrections on the isotope ratios studied for this work is discussed in detail elsewhere in this paper.

A corrective strategy employing a multielement response curve, developed and discussed in detail by previous investigators [12,37], was used in this study. NIST 1263a, a steel standard, was chosen as a matrix-matched multielement sample for this purpose. This standard contained elements across the mass range of interest in this work (up to $\mathrm{m} / \mathrm{z}=107$ ). After correcting for isotopic abundance and ionization efficiency $\left(\mathrm{T}_{\text {ion }}=7500 \mathrm{~K}, \mathrm{n}_{\mathrm{e}}=1 \mathrm{x} 10^{15}\right.$

$\mathrm{cm}^{-3}$ ), a polynomial was fit to the collected data, as shown in Fig. 1. All multielement data were collected after analysis of the pure metal samples so that no residual atomic ions from ablation of the standard were available to interfere with measurement of $\mathrm{MAr}^{+}$ion abundances.

\section{Results and Discussion}

The $\mathrm{MAr}^{+} / \mathrm{M}^{+}(\mathrm{M}=\mathrm{Ti}, \mathrm{V}, \mathrm{Cr}, \mathrm{Mn}, \mathrm{Fe}, \mathrm{Co}, \mathrm{Ni}, \mathrm{Cu}$ and $\mathrm{Zn})$ signal ratio was measured for nine first-row transition metals and is presented uncorrected for mass bias effects in Table 3. The signal ratios in the table are multiplied by $10^{6}$ and are thus presented in ppm for convenient comparison. They are comparable to those measured by Becker et al. [17] and by Guillong et al. [14] for several of the same $\mathrm{MAr}^{+}$ions. The ratios found by Guillong et al. [14], included for comparison in Table 3, provide particularly suitable points of reference for the $\mathrm{MAr}^{+} / \mathrm{M}^{+}$ratios in this study, as they were collected on the same model of ICP-MS (XSeries 2). Their measured ratios are higher than ours by factors of 5 to 8 . Some of this disparity can be attributed to the use of both a different mass bias correction method and 
cones of a different geometry than those in our study. Despite this, the $T_{\text {gas }}$ values calculated from the ratios published by Guillong et al. describe a fate similar to that found in this work for $\mathrm{MAr}^{+}$ions, as will be discussed.

The low abundance of each $\mathrm{MAr}^{+}$ion relative to the corresponding $\mathrm{M}^{+}$ion ( 1 to 10 ppm, $\sim 10^{-6}$ to $10^{-5}$ ) is expected for this type of polyatomic ion. Even under plasma conditions, the formation of argon-based diatomics with metals is not highly favored energetically. Experimentally and theoretically determined dissociation energies for MAr ${ }^{+}$ ions incorporating transition metals (Table 1) [20-31] are no higher than $0.55 \mathrm{eV}$, which illustrates the weakly-bound nature of these ions. Despite this, the absolute abundance of $\mathrm{MAr}^{+}$interferences can still have a significant impact on trace element detection when $\mathrm{M}$ represents a matrix element.

A general trend of decreasing dissociation energies from Ti to Fe, followed by a marked increase centered on $\mathrm{Ni}$, has been found for transition metal-based $\mathrm{MAr}^{+}$ions. Because these values are collected from several sources, discrepancies in the degree of change in $\mathrm{D}_{0}$ values between species, and even exceptions to the trend (e.g., $\mathrm{VAr}^{+}$), are found. Nonetheless, a broad correlation is observed between increasing $\mathrm{MAr}^{+} / \mathrm{M}^{+}$ratios and increasing dissociation energies across the period of the first-row transition metals. Both the upper and lower bounds of $\mathrm{MAr}^{+} / \mathrm{M}^{+}$ratios are established by the most weakly $\left(\mathrm{FeAr}^{+}\right)$and strongly $\left(\mathrm{NiAr}^{+}\right)$bound ions, based on experimentally determined $\mathrm{D}_{0}$ values.

Differences in the dissociation energies among $\mathrm{MAr}^{+}$ions may be influenced by both the electronegativity of the transition metal and electron configuration of the metal ion. It has been suggested that a more covalent-type interaction occurs between the metal ion and argon neutral for metals with higher electronegativities [17]. While this is a very weak 
covalent interaction for all transition metals with argon, it would account for the higher $\mathrm{MAr}^{+} / \mathrm{M}^{+}$ion ratios calculated for metals toward the right end of the period in the periodic table, where the greatest electronegativities are found. Indeed the most electronegative of the metals studied, Ni (Pauling electronegativity $=1.8$ ), shows the highest relative abundance of $\mathrm{MAr}^{+}$ions.

However, electronegativity patterns alone cannot fully account for the variation in the binding strength of the $\mathrm{MAr}^{+}$ions. If this trend were the sole controlling factor, the $\mathrm{D}_{0}$ values for $\mathrm{FeAr}^{+}(0.11 \mathrm{eV})$ and $\mathrm{CoAr}^{+}(0.51 \mathrm{eV})$ should be similar due to identical electronegativity values (Pauling electronegativity $=1.7$ for both), but they are quite different. The $\mathrm{MAr}^{+} / \mathrm{M}^{+}$signal ratios measured for these species bear out the difference in binding strength, with the $\mathrm{CoAr}{ }^{+} / \mathrm{Co}^{+}$ratio approximately an order of magnitude greater than the $\mathrm{FeAr}^{+} / \mathrm{Fe}^{+}$ratio. The cause of the weaker interaction between the $\mathrm{Fe}$ and $\mathrm{Ar}$ atoms, as compared to Co and Ar atoms, is likely related to the electron configuration of the metal ion.

Given the low ionization energy of most of the transition metals (6.7 to $7.9 \mathrm{eV}, 9.29$ $\mathrm{eV}$ for $\mathrm{Zn})$ against that of $\operatorname{argon}(15.76 \mathrm{eV})$, it is expected that the formation of $\mathrm{MAr}^{+}$ions results from the interaction of an $\mathrm{M}^{+}$ion with neutral argon. Thus one of the factors in the reaction is the electronic state of the $\mathrm{M}^{+}$ion. The valence electron configuration of most first-row transition metals (Table 4) [38-40] includes only electrons in the $3 \mathrm{~d}$ orbitals. Theoretical modeling has established that the absence of electrons in the $4 \mathrm{~s}$ subshell reduces repulsion between metal ions and argon, enhancing the strength of the $\mathrm{MAr}^{+}$ion $[20,24]$.

Those transition metal ions with 4 s electrons $\left(\mathrm{Mn}^{+}, \mathrm{Fe}^{+}\right)$form much weaker diatomic ions with argon than nearby elements with empty 4 s subshells. Both $\mathrm{MnAr}^{+}$and $\mathrm{FeAr}^{+}$were less abundant relative to their respective $\mathrm{M}^{+}$ions than the other $\mathrm{MAr}^{+}$ions measured. $\mathrm{TiAr}^{+}$ 
appears to be an exception to this rule; the $\operatorname{TiAr}^{+}$cation has a more complex ground state electron configuration. Though the lowest energy and preferred configuration places an electron in the $4 \mathrm{~s}$ subshell, a low lying electronic state exists with no electrons in the $4 \mathrm{~s}$ subshell [20]. This state likely contributes to the population of $\mathrm{Ti}^{+}$ions in the ICP, forming a more stable diatomic ion with argon than would be otherwise expected from the lowest energy configuration alone.

$\mathrm{T}_{\text {gas }}$ values (Table 3) were determined for each $\mathrm{MAr}^{+}$ion where all necessary spectroscopic values (Table 1) were available. Very high $\mathrm{T}_{\text {gas }}$ values that are very different for the various ions are found for the $\mathrm{MAr}^{+}$ions chosen, with even the lowest value $(\sim 8000$ $\mathrm{K}_{\text {for }} \mathrm{NiAr}^{+}$) laying clearly above the typical $\mathrm{T}_{\text {gas }}$ range of the ICP (5000 to $6000 \mathrm{~K}$ ). This observations indicate a) these polyatomic ions are much less abundant in the mass spectrum relative to the metal atomic ion than would be predicted based upon plasma conditions alone, and $\mathrm{b}$ ) the lack of a uniform $\mathrm{T}_{\text {gas }}$ value shows their relative abundances are far out of thermal equilibrium. Thus, some process(es) during ion extraction greatly reduce the abundance of MAr relative to $\mathrm{M}^{+}$.

The $\mathrm{MAr}^{+} / \mathrm{M}^{+}$ratios discussed have been presented without the mass bias corrections typically applied to isotope ratios calculated from ICP-MS spectral data. The basis for such corrections rests in constructing a plot of instrument response over a range of mass-to-charge values covering the isotope masses of interest. Instrument response is calculated by adjusting the measured atomic ion signal for isotopic abundance, ionization efficiency in the ICP and concentration in the multielement standard used. From this the preferential transmission of heavier isotopes, often attributed chiefly to space charge effects that cause greater repulsive losses of lighter ions [41], can be corrected in isotope ratio calculations. This approach 
should be generally valid when correcting atomic ion ratios, whose behavior is represented by the atomic ions used to create the instrument response curve.

For lack of something better, this same mass bias correction approach has been used previously for polyatomic ions. It is not clear that it is valid when the measured signal ratios and $\mathrm{T}_{\text {gas }}$ values show clearly that the polyatomic ions are attenuated during ion extraction. Additional factors beyond space charge effects could alter the observed ion abundances. In particular, the instrument response curve from atomic ions cannot account for the dissociation of $\mathrm{MAr}^{+}$ions during extraction. For example, the instrument response curve created for this study (Fig. 1) would predict that the polyatomic ion ${ }^{64} \mathrm{Zn}^{40} \mathrm{Ar}^{+}$would be transmitted at $3 \mathrm{x}$ the level of the lighter ${ }^{64} \mathrm{Zn}^{+}$atomic ion, based on the loss of ions at $\mathrm{m} / \mathrm{z}=$ 64 relative to those at $\mathrm{m} / \mathrm{z}=104$. The mass difference between any $\mathrm{MAr}^{+}$ion and its $\mathrm{M}^{+}$ analog is $40 \mathrm{Da}$, the highest value for any abundant polyatomic ions and thus subject to the largest numerical mass bias correction. If, however, $\mathrm{MAr}^{+}$ions are dissociated to a very high degree during extraction, it is unlikely that the heavier polyatomic ion is being transmitted at that great a level relative to the atomic ion.

Uncertainty as to the proper exercise of mass bias corrections would seem to be a significant hurdle in the use of $\mathrm{MAr}^{+} / \mathrm{M}^{+}$ion ratios for the determination of $\mathrm{T}_{\text {gas }}$ and polyatomic ion origins. Given the large relative mass difference between the ions, application of mass bias corrections to $\mathrm{MAr}^{+} / \mathrm{M}^{+}$ion ratios greatly influences the value calculated (Table 3). The calculated ratios are reduced by factors of $\sim 3$ with these corrections, which makes the measured $\mathrm{T}_{\text {gas }}$ values higher than $6000 \mathrm{~K}$ by even greater amounts. Ergo, doubt about the validity of mass bias corrections does not affect the assignment of ion origins, which is the primary goal of the work. The determined $\mathrm{T}_{\text {gas }}$ values 
still indicate extensive removal of $\mathrm{MAr}^{+}$ions during ion extraction. Application of this mass bias model to the ion ratios calculated in this study does not alter the conclusion that processes during ion extraction reduce the abundance of $\mathrm{MAr}^{+}$ions.

For weakly bound $\mathrm{MAr}^{+}$ions, collisions in the regions after the sampler and/or skimmer could readily result in the fragmentation of the polyatomic ion. Such a mechanism is in line with the proposal put forth by Guillong et al. that flow restrictions through the sampler and skimmer cones promote collisions that dissociate $\mathrm{MAr}^{+}$ions [14]. We also provide $\mathrm{T}_{\text {gas }}$ values from their measurements of $\mathrm{MAr}^{+} / \mathrm{M}^{+}$ions with an XSeries 2, the same device used in this study, in Table 3. Even with relative $\mathrm{MAr}^{+}$abundances greater than those found in this study, all $\mathrm{T}_{\text {gas }}$ values are still much higher than $6000 \mathrm{~K}$, again indicating extensive removal of these ions during extraction. For the $\mathrm{MAr}^{+}$ions to be present in the mass spectrum as expected based on plasma conditions, the relative abundance of $\mathrm{MAr}^{+}$ions would need to be 1 to 3 orders of magnitude higher than those observed experimentally (Table 5). If it is assumed that the observed $\mathrm{MAr}^{+}$ion signal represents the fraction that survives from the plasma, up to $99 \%$ of transition metal $\mathrm{MAr}^{+}$ions are dissociated during ion extraction.

These considerations clearly indicate that processes occurring during ion extraction actually remove most of the $\mathrm{MAr}^{+}$ions. This observation is somewhat surprising given that fundamental studies of these ions use supersonic expansions for the reverse objective - to make them. The early predictions of Douglas [42] are borne out once again, that the high temperature of the ICP acts to suppress the abundances of weakly-bound polyatomic ions. In our opinion, this extensive dissociation of $\mathrm{MAr}^{+}$ions may also be fostered by the existence of a small potential between the plasma and the mass spectrometer interface. In 
this experiment, a grounded metal shield is used between the load coil and the outside of the torch. The free electrons in the metal shield prevent the potential on the load coil from coupling capacitively to the plasma [43-45]. However, the shield does not cover the entire outside of the torch; a small gap ( $2.2 \mathrm{~mm}$ wide) is left to prevent buildup of circulating currents that would otherwise melt the shield [43]. Thus, a small potential may be induced in the plasma from the voltage gradient down the load coil due to this gap. Such a potential can readily penetrate into the region between the sampler and skimmer, as shown by old Langmuir probe measurements [46]. As ions travel between the plasma and the sampler, or between the sampler and skimmer, they gain energy from this potential and also collide with background Ar gas atoms. The situation is analogous to that encountered with a nozzleskimmer extaction system in electrospray MS; the skimmer can be biased at a potential more negative than that of the nozzle to induce fragmentation of positive ions [47]. A potential of only 2 volts would result in a center-of-mass collision energy of $2 \mathrm{eV} *\left[\mathrm{~m}_{\mathrm{Ar}} /\left(\mathrm{m}_{\mathrm{Ar}}+\mathrm{m}_{\mathrm{MAr}+}\right)\right]$ $\sim 0.8 \mathrm{eV}$, well above the dissociation energies of any of the $\mathrm{MAr}^{+}$ions $\left(\mathrm{D}_{0}=0.1\right.$ to $\left.0.55 \mathrm{eV}\right)$ examined in this study. Given the high $\mathrm{T}_{\text {gas }}$ values found for other weakly-bound polyatomic ions, such as $\mathrm{ArO}^{+}$[48], removal of weakly-bound polyatomic ions through collisions induced by the plasma potential may actually remove many polyatomic ion interferences and thus be desirable.

\section{Conclusions}

Measured $\mathrm{MAr}^{+} / \mathrm{M}^{+}$ion ratios from ablation of pure transition metals are similar to those determined experimentally by other investigators for these species in LA-ICP-MS. A general correlation is observed between the calculated ion ratios and the dissociation energy 
of each $\mathrm{MAr}^{+}$ion. Transition metal ions with one or more $4 \mathrm{~s}$ electrons in the ground state configuration form the most weakly-bound $\mathrm{MAr}^{+}$ions, as the $\mathrm{MnAr}^{+} / \mathrm{Mn}^{+}$and $\mathrm{FeAr}^{+} / \mathrm{Fe}^{+}$ ratios illustrate. Contributions from an empty $4 \mathrm{~s}$ subshell configuration to the ground state of $\mathrm{Ti}^{+}$stabilize the $\mathrm{TiAr}^{+}$ion, resulting in a larger $\mathrm{MAr}^{+} / \mathrm{M}^{+}$ratio than for $\mathrm{MnAr}^{+}$and $\mathrm{FeAr}^{+}$ ions.

$\mathrm{T}_{\text {gas }}$ values much greater than $6000 \mathrm{~K}$ are found for all transition metal-based $\mathrm{MAr}^{+}$ ions studied, indicating very extensive dissociation of the polyatomic ions during ion extraction. Collisions during the extraction process may actually remove most of the weaklybound $\mathrm{MAr}^{+}$ions relative to the level expected based on plasma conditions. Multiple aspects of the ion extraction process could be responsible for these collisions. Flow restrictions through the orifice of the sampler and skimmer cones could increase the number of collisions as the ions are sampled from the plasma. A small plasma potential may exist due to incomplete shielding of the ICP by the guard electrode. Though small, this plasma potential may nonetheless be strong enough to bring about collisions of sufficient energy to dissociate $\mathrm{MAr}^{+}$ions during extraction. Evidence of the attenuation of weakly-bound ions by such a potential may also be found in the very high $\mathrm{T}_{\text {gas }}$ values consistently measured for $\mathrm{ArO}^{+}$[48]. Ion kinetic energy distributions could be studied to establish whether such a plasma potential exists for shielded plasmas. This information could prove valuable not only to understanding those processes related to $\mathrm{MAr}^{+}$ion abundances but to the general knowledge of ion extraction conditions on current ICP-MS instrumentation as well. 


\section{Acknowledgements}

This research was supported by the U. S. Department of Energy, Office of Nuclear Nonproliferation (NA-22) and the Office of Basic Energy Sciences. The XSeries 2 ICP-MS was purchased with funds provided by the U. S. Department of Energy, Office of Nuclear Nonproliferation (NA-22) and the Office of Basic Energy Sciences. The authors thank Larry Jones for providing the transition metal samples. The Ames Laboratory is operated for the U. S. Department of Energy by Iowa State University under Contract No. DE-AC02$07 \mathrm{CH} 11358$.

\section{References}

[1] R.S. Houk, V.A. Fassel, G.D. Flesch, H.J. Svec, A.L. Gray, C.E. Taylor, Inductively coupled argon plasma as an ion source for mass spectrometric determination of trace elements, Anal. Chem. 52 (1980) 2283-2289.

[2] K.E. Jarvis, A.L. Gray, R.S. Houk, Handbook of Inductively Coupled Plasma Mass Spectrometry (Chapman and Hall, New York, 1992).

[3] G.A. Meyer, R.M. Barnes, Analytical inductively coupled nitrogen and air plasmas, Spectrochim. Acta Part B 40 (1985) 893-905.

[4] H. Uchida, T. Ito, Inductively Coupled Nitrogen Plasma Mass Spectrometry Assisted by Adding Argon to the Outer Gas, J. Anal. At. Spectrom. 10 (1995) 843-848.

[5] P. Yang, R.M. Barnes, A low-power oxygen inductively coupled plasma for spectrochemical analysis--IV. Analytical features, Spectrochim. Acta Part B 45 (1990) 197-176.

[6] H. Hayashi, M. Hiraide, Low-pressure helium ICP-MS for trace analysis, Bunseki Kagaku 53 (2004) 793-804.

[7] T. Nagayasu, H. Hayashi, M. Hiraide, Laser Ablation and Low-Pressure Helium-ICPMS for the Analysis of Alumina Powder Dispersed in Glycerol, Anal. Sci. 21 (2005) 1411-1413.

[8] T. Manning, G. Bentley, B. Palmer, D. Hof, Generation of a low flow atmospheric pressure neon ICP, Spectrosc. Lett. 22 (1989) 341-344.

[9] G.A. Meyer, Determination of metals in xylene by inductively coupled air plasma emission spectrometry, Spectrochim. Acta Part B 42 (1987) 201-206.

[10] H. Niu, R.S. Houk, Fundamental aspects of ion extraction in inductively coupled plasma mass spectrometry, Spectrochim. Acta Part B 51 (1996) 779-815.

[11] R.S. Houk, N. Praphairaksit, Dissociation of polyatomic ions in inductively coupled plasma, Spectrochim. Acta Part B 56 (2001) 1069-1096. 
[12] J.W. Ferguson, R.S. Houk, High resolution studies of the origins of polyatomic ions in inductively coupled plasma-mass spectrometry, Part I. Identification methods and effects of neutral gas density assumptions, extraction voltage, and cone material, Spectrochim. Acta Part B 61 (2006) 905-915.

[13] S.M. McIntyre, J.W. Ferguson, T.M. Witte, R.S. Houk, Measurement of gas kinetic temperatures for polyatomic ions in inductively coupled-plasma mass spectrometry: Validation and refinements, Spectrochim. Acta Part B 66 (2011) 248-254.

[14] M. Guillong, L. Danyushevsky, M. Walle, M. Raveggi, The effect of quarupole ICPMS interface and ion lens design on argide formation. Implications for LAICPMS analysis of PGE's in geological samples, J. Anal. At. Spectrom. 26 (2011) 1401-1407.

[15] B. Hattendorf, D. Günther, M. Schönbächler, A. Halliday, Simultaneous Ultratrace Determination of $\mathrm{Zr}$ and $\mathrm{Nb}$ in Chromium Matrixes with ICP-Dynamic Reaction Cell MS, Anal. Chem. 73 (2001) 5494-5498.

[16] M.-F. Zhou, J. Malpas, M. Sun, Y. Liu, X. Fu, A new method to correct Ni- and Cuargide interference in the determination of the platinum-group elements, $\mathrm{Ru}, \mathrm{Rh}$, and Pd, by ICP-MS, Geochem. J. 35 (2001) 413-420.

[17] J.S. Becker, G. Seifert, A.I. Saprykin, H.-J. Dietze, Mass Spectrometric and Theoretical Investigations Into the Formation of Argon Molecular Ions in Plasma Mass Spectrometry, J. Anal. At. Spectrom. 11 (1996) 643-648.

[18] H.P. Longerich, Mass Spectrometric Determination of the Temperature of an Argon Inductively Coupled Plasma From the Formation of the Singly Charged Monoxide Rare Earths and their Known Dissociation Energies, J. Anal. Atom. Spectrom. 4 (1989) 491-497.

[19] E.H. Evans, L. Ebdon, L. Rowley, Comparative study of the determination of equilibrium dissociation temperature in inductively coupled plasma-mass spectrometry, Spectrochim. Acta Part B 57 (2002) 741-754.

[20] H. Partridge, C.W. Bauschlicher, Jr., Theoretical Study of the Low-Lying States of $\mathrm{TiHe}^{+}, \mathrm{TiNe}^{+}, \mathrm{TiAr}^{+}, \mathrm{VAr}^{+}, \mathrm{CrHe}^{+}, \mathrm{CrAr}^{+}, \mathrm{FeHe}^{+}, \mathrm{FeAr}^{+}, \mathrm{CoHe}^{+}$, and $\mathrm{CoAr}^{+}, \mathrm{J}$. Chem. Phys. 98 (1994) 2301-2306.

[21] C.W. Bauschlicher, Jr., H. Partridge, S.R. Langhoff, Theoretical study of metal noblegas positive ions, J. Chem. Phys. 91 (1989) 4733-4737.

[22] T. Hayes, D. Bellert, T. Buthelezi, P.J. Brucat, The bond length of $\mathrm{VAr}^{+}$, Chem. Phys. Lett. 287 (1998) 22-28.

[23] D.E. Lessen, R.L. Asher, P.J. Brucat, Spectroscopically determined binding energies of $\mathrm{CrAr}^{+}$and $\mathrm{Cr}\left(\mathrm{N}_{2}\right)^{+}$, Chem. Phys. Lett. 177 (1991) 380-382.

[24] B.L. Hammond, W.A. Lester, Jr., M. Braga, C.A. Taft, Theoretical study of the interaction of ionized transition metals $(\mathrm{Cr}, \mathrm{Mn}, \mathrm{Fe}, \mathrm{Co}, \mathrm{Ni}, \mathrm{Cu})$ with argon, Phys. Rev. A 41 (1990) 10447-10452.

[25] B.L. Tjelta, D. Walter, P.B. Armentrout, Determination of weak $\mathrm{Fe}^{+}-\mathrm{L}$ bond energies $\left(\mathrm{L}=\mathrm{Ar}, \mathrm{Kr}, \mathrm{Xe}, \mathrm{N}_{2}\right.$, and $\mathrm{CO}_{2}$ ) by ligand exchange reactions and collision-induced dissociation, Int. J. Mass Spectrom. 204 (2001) 7-21. 
[26] T. Bastug, W.-D. Sepp, B. Fricke, E. Johnson, C.M. Barshick, All-electron relativistic Dirac-Fock-Slater self-consistent-field calculations of the singly charged diatomic transition-metal- (Fe, Co, Ni, Cu, Zn) argon molecules, Phys. Rev. A 52 (1995) 27342736.

[27] R.L. Asher, D. Bellert, T. Buthelezi, P.J. Brucat, The Ground State of CoAr ${ }^{+}$, Chem. Phys. Lett. 227 (1994) 277-282.

[28] D. Lessen, P.J. Brucat, Resonant photodissociation of $\mathrm{CoAr}^{+}$and $\mathrm{CoKr}^{+}$: Analysis of vibrational structure, J. Chem. Phys. 90 (1989) 6296-6305.

[29] D. Lessen, P.J. Brucat, On the Nature of NiAr ${ }^{+}$, Chem. Phys. Lett. 152 (1988) 473476.

[30] A.W.K. Leung, D. Bellert, R.R. Julian, W.H. Breckenridge, Resonant two-color photoionization threshold measurements of the $\mathrm{Zn}^{+}$(4s) Ar bond strength: Modelpotential analysis of $\mathrm{M}^{+}$(ns) Ar interactions, J. Chem. Phys. 110 (1999) 6298-6305.

[31] C.M. Barshick, D.H. Smith, E. Johnson, F.L. King, T. Bastug, B. Fricke, Periodic Nature of Metal-Noble Gas Adduct Ions in Glow Discharge Mass Spectrometry, Appl. Spectrosc. 49 (1995) 885-889.

[32] R.E. Sonntag, G.J. Van Wylen, Fundamentals of Statistical Thermodynamics (John Wiley and Sons, Inc., New York, 1966).

[33] N.M. Laurendeau, Statistical Thermodynamics: Fundamentals and Applications, Chapter 9 (Cambridge University Press, Cambridge, 2005).

[34] L. De Galan, R. Smith, J.D. Winefordner, The electronic partition functions of atoms and ions between $1500 \mathrm{~K}$ and $7000 \mathrm{~K}$, Spectrochim. Acta Part B 23 (1968) 521-525.

[35] S. Tamaki, T. Kuroda, The electronic partition functions of atoms and ions between $7000 \mathrm{~K}$ and 12000 K, Spectrochim. Acta Part B 42 (1987) 1105-1111.

[36] T.M. Witte, Laser ablation-inductively coupled plasma-mass spectrometry: examinations of the origins of polyatomic ions and advances in the sampling of particulates, Thesis (Ph. D.)Iowa State University, 2011.

[37] C.P. Ingle, B.L. Sharp, M.S.A. Horstwood, R.R. Parrish, D.J. Lewis, Instrument response functions, mass bias, and matrix effects in isotope ratio measurements and semi-quantitative analysis by single and multi-collectore ICP-MS, J. Anal. Atom. Spectrom. 18 (2003) 219-229.

[38] B.L. Kickel, P.B. Armentrout, Guided Ion Beam Studies of the Reactions of $\mathrm{Ti}^{+}, \mathrm{V}^{+}$, and $\mathrm{Cr}^{+}$with Silane. Electronic State Effects, Comparison to Reactions with Methane, and $\mathrm{M}^{+}-\mathrm{SiH}_{\mathrm{x}}(\mathrm{x}=0-3)$ Bond Energies, J. Am. Chem. Soc. 116 (1994) 10742-10750.

[39] B.L. Kickel, P.B. Armentrout, Guided Ion Beam Studies of the Reactions of $\mathrm{Mn}^{+}$, $\mathrm{Cu}^{+}$, and $\mathrm{Zn}^{+}$with Silane. $\mathrm{M}^{+}-\mathrm{SiH}_{\mathrm{x}}(\mathrm{x}=0-3)$ Bond Energies, J. Phys. Chem-US 99 (1995) 2024-2032.

[40] B.L. Kickel, P.B. Armentrout, Reactions of $\mathrm{Fe}^{+}, \mathrm{Co}^{+}$, and $\mathrm{Ni}^{+}$with Silane. Electronic State Effects, Comparison to Reactions with Methane, and $\mathrm{M}^{+}-\mathrm{SiH}_{\mathrm{x}}(\mathrm{x}=0-3)$ Bond Energies, J. Am. Chem. Soc. 117 (1995) 764-773.

[41] G.R. Gillson, D.J. Douglas, J.E. Fulford, K.W. Halligan, S.D. Tanner, Nonspectroscopic interelement interferences in inductively coupled plasma mass spectrometry, Anal. Chem. 60 (1988) 1472-1474.

[42] D.J. Douglas, personal communication, (1996). 
[43] A.L. Gray, Communication. Influence of load coil geometry on oxide and doubly charged ion response in inductively coupled plasma source mass spectrometry, J. Anal. At. Spectrom. 1 (1986) 247-249.

[44] K.i. Sakata, K. Kawabata, Reduction of fundamental polyatomic ions in inductively coupled plasma mass spectrometry, Spectrochim. Acta Part B 49 (1994) 1027-1038.

[45] N.S. Nonose, N. Matsuda, N. Fudagawa, M. Kubota, Some characteristics of polyatomic ion spectra in inductively coupled plasma mass spectrometry, Spectrochim. Acta Part B 49 (1994) 955-974.

[46] H.B. Lim, R.S. Houk, J.S. Crain, Langmuir probe measurements of potential inside a supersonic jet extracted from an inductively coupled plasma, Spectrochim. Acta Part B 44 (1989) 989-998.

[47] B.B. Schneider, D.D.Y. Chen, Collision-Induced Dissociation of Ions with the Orifice-Skimmer Region of an Electrospray Mass Spectrometer, Anal. Chem. 72 (2000) 791-799.

[48] S.M. McIntyre, J.W. Ferguson, R.S. Houk, Determination of dissociation temperature for $\mathrm{ArO}^{+}$in inductively coupled plasma-mass spectrometry: Effects of excited electronic states and dissociation pathways, Spectrochim. Acta Part B 66 (2011) 581587. 
Table 1. Dissociation Energies and Spectroscopic Data for First-Row Transition Metal Argide $\left(\mathrm{MAr}^{+}\right)$Ions. Both theoretical and experimental values are listed where available. The $\mathrm{D}_{0}$ values actually used are indicated with asterisks.

\begin{tabular}{|c|c|c|c|c|c|c|c|}
\hline & & $\begin{array}{c}\mathrm{D}_{0} \\
(\mathrm{eV})\end{array}$ & $\begin{array}{c}\omega \\
\left(\mathrm{cm}^{-1}\right)\end{array}$ & $\begin{array}{c}B \\
(\mathrm{~cm}-1)\end{array}$ & g & $\begin{array}{c}\text { Term } \\
\text { Symbol }\end{array}$ & Refs. \\
\hline $\mathrm{TiAr}^{+}$ & Theo & $0.31^{\star}$ & 163 & 0.110 & 8 & ${ }^{4} \Phi$ & [20] \\
\hline \multirow[t]{2}{*}{$\mathrm{VAr}^{+}$} & Theo & 0.291 & 143 & 0.103 & 5 & ${ }^{5} \Sigma$ & {$[21,22]$} \\
\hline & Exp & $0.369^{\star}$ & & 0.108 & & & [22] \\
\hline \multirow[t]{2}{*}{$\mathrm{CrAr}^{+}$} & Theo & 0.239 & 111 & 0.085 & 6 & ${ }^{6} \boldsymbol{\Sigma}$ & [20] \\
\hline & $\operatorname{Exp}$ & $0.29^{\star}$ & & & & & [23] \\
\hline $\mathrm{MnAr}^{+}$ & Theo & $0.149^{\star}$ & 93 & 0.090 & 7 & ${ }^{7} \boldsymbol{\Sigma}$ & [24] \\
\hline \multirow[t]{2}{*}{$\mathrm{FeAr}^{+}$} & Theo & 0.493 & 198 & 0.116 & 12 & ${ }^{6} \Delta$ & {$[25,26]$} \\
\hline & Exp & $0.11^{*}$ & 98 & 0.092 & & & [25] \\
\hline \multirow[t]{2}{*}{$\mathrm{CoAr}^{+}$} & Theo & 0.533 & 199 & 0.123 & 6 & ${ }^{3} \Delta$ & {$[27,26]$} \\
\hline & Exp & $0.51^{*}$ & 265 & 0.124 & & & {$[27,28]$} \\
\hline \multirow[t]{2}{*}{$\mathrm{NiAr}^{+}$} & Theo & 0.52 & 195 & 0.123 & 2 & ${ }^{2} \boldsymbol{\Sigma}$ & {$[24,26]$} \\
\hline & Exp & $0.55^{\star}$ & 235 & 0.165 & & & [29] \\
\hline $\mathrm{CuAr}^{+}$ & Theo & $0.53^{\star}$ & 197 & 0.119 & 1 & ${ }^{1} \Sigma$ & {$[24,26]$} \\
\hline \multirow[t]{2}{*}{$\mathrm{ZnAr}^{+}$} & Theo & 0.322 & 132 & 0.101 & 2 & ${ }^{2} \Sigma$ & {$[26,30,31]$} \\
\hline & Exp & $0.25^{\star}$ & & & & & [30] \\
\hline
\end{tabular}


Table 2. LA-ICP-MS Operating Parameters

\begin{tabular}{ll}
\hline ICP-MS Instrument & Thermo XSeries 2 \\
Torch & Shielded (grounded Pt guard electrode) \\
Interface Configuration & Xt (Ni sampler and skimmer cones) \\
RF Power & $1400 \mathrm{~W}$ \\
Outer Gas & $13 \mathrm{~L} \mathrm{~min}^{-1}$ \\
Auxiliary Gas & $0.7 \mathrm{~L} \mathrm{~min}^{-1}$ \\
Aerosol Gas & $1.0 \mathrm{~L} \mathrm{~min}^{-1}$ \\
Detector Mode & Dual (analog and pulse counting) \\
& Cross-calibrated \\
Laser System & LSX-500 Nd:YAG $266 \mathrm{~nm}$ \\
Energy & $8.65 \mathrm{~mJ}$ \\
Spot Size & $150 \mu \mathrm{m}$ \\
Repetition Rate & $10 \mathrm{~Hz}$ \\
Ablation Mode & Raster $\left(100 \mu \mathrm{m} \mathrm{s}^{-1}\right)$ \\
\hline
\end{tabular}


Table 3. $\mathrm{MAr}^{+} / \mathrm{M}^{+}$Ion Ratios (in ppm) and $\mathrm{T}_{\text {gas }}$ Values for First-Row Transition Metals

\begin{tabular}{cccccccccccc}
\hline & \multicolumn{2}{c}{$\mathrm{FeAr}^{+}$} & \multicolumn{2}{c}{$\mathrm{MnAr}^{+}$} & \multicolumn{2}{c}{$\mathrm{CrAr}^{+}$} & \multicolumn{2}{c}{$\mathrm{ZnAr}^{+}$} & \multicolumn{3}{c}{$\mathrm{TiAr}^{+}$} \\
Mass Bias & No & Yes & $\mathrm{N}$ & $\mathrm{Y}$ & $\mathrm{N}$ & $\mathrm{Y}$ & $\mathrm{N}$ & $\mathrm{Y}$ & $\mathrm{N}$ & $\mathrm{Y}$ \\
\hline $\begin{array}{c}\text { This Study } \\
\mathrm{MAr}^{+} / \mathrm{M}^{+}\end{array}$ & 0.538 & 0.168 & 0.607 & 0.186 & 3.19 & 0.93 & 7.88 & 2.78 & 8.13 & 2.19 \\
& & & & & & & & & & & \\
$\mathrm{~T}_{\text {gas }}(\mathrm{K})$ & 73700 & 112000 & 141000 & 227000 & 30800 & 47100 & 87200 & 130000 & 15300 & 34500
\end{tabular}

Guillong et al.

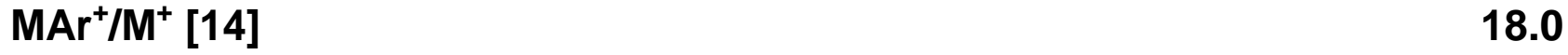

\begin{tabular}{ll}
$\mathrm{T}_{\text {gas }}(\mathrm{K})$ & 60300 \\
\hline
\end{tabular}

\begin{tabular}{cccccccccc}
\hline & \multicolumn{2}{c}{$\mathrm{VAr}^{+}$} & \multicolumn{2}{c}{$\mathrm{CoAr}^{+}$} & \multicolumn{2}{c}{$\mathrm{CuAr}^{+}$} & \multicolumn{2}{c}{$\mathrm{NiAr}^{+}$} \\
Mass Bias & $\mathrm{N}$ & $\mathrm{Y}$ & $\mathrm{N}$ & $\mathrm{Y}$ & $\mathrm{N}$ & $\mathrm{Y}$ & $\mathrm{N}$ & $\mathrm{Y}$ \\
\hline $\begin{array}{c}\text { This Study } \\
\mathrm{MAr}^{+} / \mathrm{M}^{+}\end{array}$ & 1.35 & 0.385 & 4.67 & 1.53 & 9.18 & 3.06 & 12.5 & 4.04 \\
& & & & & & & & \\
$\mathrm{~T}_{\text {gas }}(\mathrm{K})$ & 32200 & 62000 & 13700 & 18400 & 17100 & 22400 & 8290 & 14300
\end{tabular}

Guillong et al.

$\mathrm{MAr}^{+} / \mathrm{M}^{+}[14]$

$13.0 \quad 20.0$

$T_{\text {gas }}(K)$

9290

15100

6740 
Table 4. Valence Electron Configuration of First-Row Transition Metal Cations

$$
\begin{array}{llllllllllll}
\text { Ion } & \mathrm{Ti}^{+} & \mathrm{V}^{+} & \mathrm{Cr}^{+} & \mathrm{Mn}^{+} & \mathrm{Fe}^{+} & \mathrm{Co}^{+} & \mathrm{Ni}^{+} & \mathrm{Cu}^{+} & \mathrm{Zn}^{+}
\end{array}
$$

Valence $\quad 3 d^{2} 4 s^{1} \quad 3 d^{4} \quad 3 d^{5} \quad 3 d^{5} 4 s^{1} \quad 3 d^{6} 4 s^{1} \quad 3 d^{8} \quad 3 d^{9} \quad 3 d^{10} \quad 3 d^{10} 4 s^{1}$ Configuration $\left(3 \mathrm{~d}^{3}\right)$

Refs.

$[20,38] \quad[38]$

[38]

[39]

[40]

[40] [40]

[39] [39] 
Table 5. Comparison of Measured $\mathrm{MAr}^{+} / \mathrm{M}^{+}$Ratios and Expected Ratios from Plasma Conditions $\left(\mathrm{T}_{\text {gas }}=6000 \mathrm{~K}\right)$

(All ratios in ppm)

\begin{tabular}{|c|c|c|c|c|}
\hline & $\begin{array}{c}D_{0} \\
(e V)\end{array}$ & $\begin{array}{c}\text { Measured } \\
\mathrm{MAr}^{+} / \mathrm{M}^{+} \text {Signal } \\
\text { Ratio } \\
\end{array}$ & $\begin{array}{l}\mathrm{MAr}^{+} / \mathrm{M}^{+} \text {Signal } \\
\text { Ratio at } 6000 \mathrm{~K}\end{array}$ & $\%$ Difference \\
\hline $\mathrm{FeAr}^{+}$ & 0.11 & 0.538 & 58.1 & 99.1 \\
\hline $\mathrm{MnAr}^{+}$ & 0.149 & 0.607 & 295 & 99.8 \\
\hline $\mathrm{ZnAr}^{+}$ & 0.25 & 7.88 & 274 & 97.1 \\
\hline $\mathrm{CrAr}^{+}$ & 0.29 & 3.19 & 314 & 99.0 \\
\hline TiAr $^{+}$ & 0.31 & 8.13 & 32.7 & 75.1 \\
\hline $\mathrm{VAr}^{+}$ & 0.369 & 1.35 & 31.2 & 95.7 \\
\hline $\mathrm{CoAr}^{+}$ & 0.51 & 4.67 & 31.5 & 85.2 \\
\hline $\mathrm{CuAr}^{+}$ & 0.53 & 9.18 & 229 & 96.0 \\
\hline $\mathrm{NiAr}^{+}$ & 0.55 & 12.5 & 26.3 & 52.5 \\
\hline
\end{tabular}




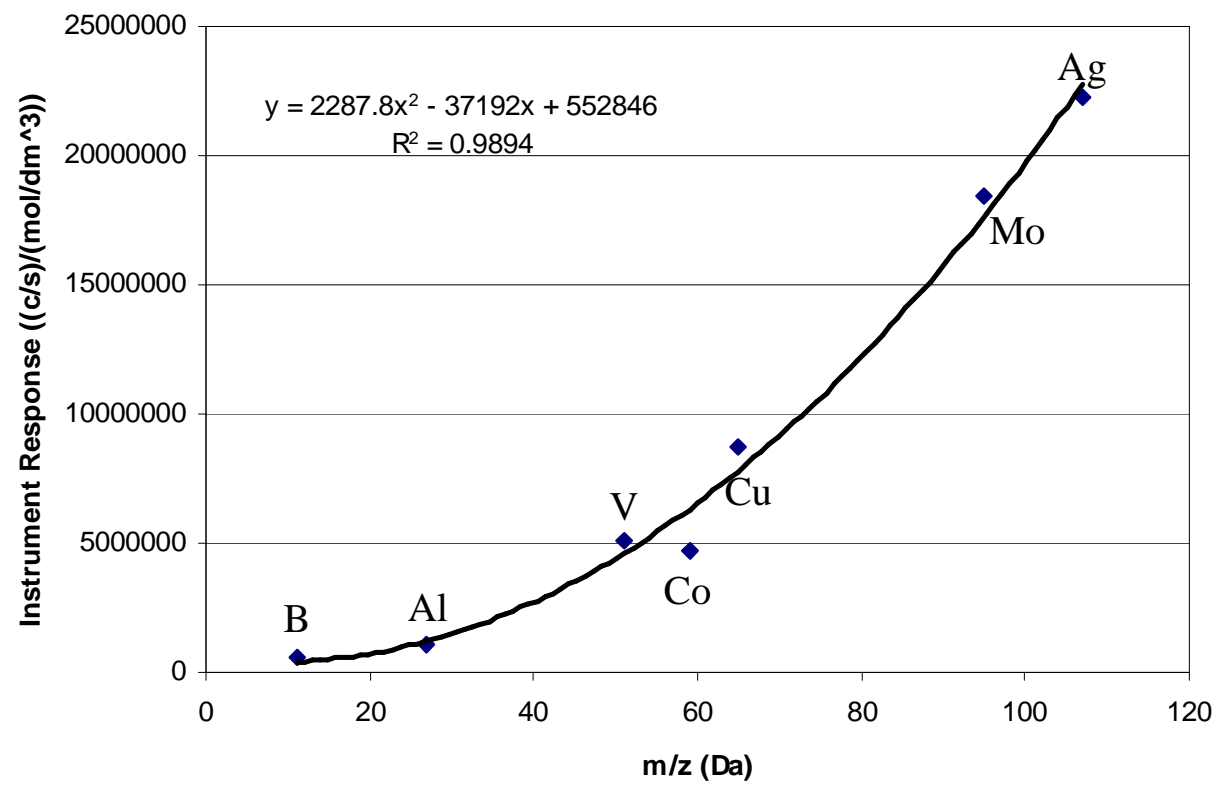

Fig. 1. Instrument Response Plot from Ablation of NIST 1263a Steel for Mass Bias Corrections 


\title{
Chapter 4. Determination of Uranium Isotope Ratios in Particulate Samples by fs-LA-ICP-MC-MS
}

Travis Witte, Daniel S. Zamzow, Cole Hexel, Debra A. Bostick, Eddie H. McBay, Stanley J. Bajic, David P. Baldwin and R. S. Houk

\begin{abstract}
Femtosecond laser ablation (fs-LA) inductively coupled plasma multicollector mass spectrometry (ICP-MC-MS) is used to determine ${ }^{235} \mathrm{U} /{ }^{238} \mathrm{U}$ ratios in particulate samples. Standard uranium oxide samples at different total mass loadings are analyzed to establish the accuracy and precision of isotope ratios measured by this technique. Examination of environmental samples shows the ability of fs-LA-ICP-MC-MS to provide information on the spatial distribution of different isotope ratios across the sample. These results demonstrate the fitness of this technique as both a supplement and substitute for traditional wet chemical methods for the determination of isotope ratios in particulate samples.
\end{abstract}

\section{Introduction}

Laser ablation inductively coupled plasma-mass spectrometry (LA-ICP-MS) provides the ability to both rapidly screen samples [1-4] and produce high-precision isotopic measurements [5-11]. Solid samples can be ablated and introduced to the ICP-MS with little sample preparation. In contrast with solution nebulization ICP-MS, which generally requires complete digestion of the sample, a quicker turnaround is achieved from sampling to 
analysis. For high volume sample analyses, this translates into a significant time-savings over the course of a sample group. LA-ICP-MS also preserves spatial information about the sample that would otherwise be lost when digestion is performed [12-16]. Ablation analysis can quickly screen samples, identifying those that contain high concentrations of the elements of interest that require thorough isotopic characterization.

As a well established technique for obtaining precise and accurate isotopic information, solution nebulization is commonly used for this more rigorous stage of the analysis [17-20]. Advances in laser systems, however, have made LA-ICP-MS a viable alternative for isotopic characterization of these samples. Since the first coupling of laser ablation sampling with ICP-MS [21], nanosecond (ns) lasers have proven effective for rapid analysis of a variety of solid sample matrices [22]. While this technique delivers a greater ease of analysis through simplified sample handling, additional factors arise that prevent nsLA-ICP-MS from presenting the same levels of accuracy and precision as solution-based analyses. These factors are related to the interaction of the laser pulse with the sample surface and the ablated material.

For many materials the ns pulse duration is a sufficient time period for the sample surface to be heated by the laser beam [23]. Sample heating has a detrimental effect on the aerosol produced by the ablation. The risk of fractionation increases as more volatile elements are preferentially removed by the thermal effects of the ablation, impacting the accuracy of the analysis [24]. Melting caused by the interaction of the ns pulse with the sample also negatively affects the ablation aerosol. Material melted by the laser can form large particles that cannot be efficiently vaporized in the ICP [25]. Large spikes in the signal can result, impairing the precision of the data. Moreover melting around the ablation crater 
can prevent material from being removed in the aerosol at all, simply redepositing it on the sample surface. This deposited material often does not have the same composition as the original sample. If it is ablated subsequently, errors result.

The introduction of femtosecond (fs) lasers greatly reduces these concerns from the analysis. The time period of the $\sim 100 \mathrm{fs}$ pulse does not permit thermal interaction between the beam and sample surface [26,27]. Ablated material is ejected from the surface before heating, alleviating the problems caused by preferential vaporization and melting from the sample. Compared with ns lasers, ablation with a fs laser produces a smaller average particle size in the ablation aerosol and a more complete vaporization of the ablated particles in the ICP $[28,29]$. The result is a more accurate representation of the solid sample composition in the mass spectrum with analytical figures of merit nearer to those achieved in solution-based analyses [30-32].

Further improvement can be gained by pairing the fs laser system with an ICP-multicollector (MC)-mass spectrometer, which allows for the simultaneous measurement of multiple isotopes [33-36]. Single collector scanning instruments can only measure one isotope at a time, limiting their utility for isotopic analysis. Signal variations measured for one isotope can not be corrected in all other isotope signals. Concurrent detection of isotopes ensures that signal variations coming from the ICP are accounted for in all ion signals, enhancing the precision of observed isotope ratios. MC-equipped ICP-MS are well-suited for analyses where precise ratios permit determination of elemental provenance based on discrimination between isotopic ratios.

This work applies fs-LA-ICP-MC-MS to the measurement of U in particulate samples. These deposited particulates represent a departure from the usual class of samples 
analyzed by LA-ICP-MS, macroscopic solids. The analysis of discrete micron-sized particulates is an area with many possible applications for LA-ICP-MS analysis. Whether for synthetic particles or collected environmental particulates, elemental and isotopic composition can be determined on a single particle basis. By combining this technique with microscopy, size and morphology-related enrichment effects could be studied. Assays of the particle to particle homogeneity within batches of synthesized particulates could also utilize single particle LA analysis.

In this study, $\mathrm{U}$ isotope ratios are measured from the ablation of particles immobilized on a cotton swipe. Reference values for the materials ablated were used to evaluate the accuracy and precision of this technique. Environmental samples of particulates of variable composition collected on single swipes were analyzed using this technique. Assessment of the spatial resolution afforded by the fs laser was also made from the collected data. The fitness of the MC-ICP-MS device for the transient signals produced by sample ablation was taken under examination with regard to the detector's ability to respond to rapidly changing signal intensity.

\section{Experimental}

\section{Instrumentation}

Ablation experiments were conducted using a Coherent Libra Ti:Sapphire fs-laser system with a home-built harmonics box and ablation chamber, permitting operation in a fully enclosed environment. The laser was operated at a wavelength of $266 \mathrm{~nm}$, which was accomplished by frequency-tripling the fundamental beam. The emitted laser featured a pulse width of approximately $100 \mathrm{fs}$, pulse energy of $120 \mu \mathrm{J}$ per pulse and a $1 \mathrm{kHz}$ repetition 
rate. Neutral density filters were used to reduce the laser power when necessary. A spot size of $35 \mu \mathrm{m}$ diameter was maintained for all ablations.

Analyses were conducted in single-spot and raster ablation modes. During single spot analyses, discrete bursts of 100 laser shots were used by opening the laser shutter for $100 \mathrm{~ms}$. Raster analyses, where the sample is moved relative to the laser spot to ablate across the sample surface, were performed using two different patterns. Pattern A consisted of moving the sample at $0.1 \mathrm{~mm} \mathrm{~s}^{-1}$ in a zig-zag pattern of five $1 \mathrm{~mm}$ lines spaced $0.2 \mathrm{~mm}$ apart, covering a $1 \mathrm{~mm}$ square. Laser pulse energy was reduced to $\sim 20 \mu \mathrm{J}$ with a neutral density filter for the ablation, which lasted nearly 1 minute. The sample was moved more quickly in Pattern B, at $0.4 \mathrm{~mm} \mathrm{~s}^{-1}$, and over a larger area, ten $2 \mathrm{~mm}$ lines spaced $0.2 \mathrm{~mm}$ apart in a 2 mm square, in the same zig-zag pattern. A higher laser energy of $\sim 60 \mu \mathrm{J}$ was used for this raster pattern. These settings increased the observed signal relative to pattern A. Pattern B was used for samples where ablation with pattern A produced signals too low to permit calculation of isotopic ratios.

The laser system was coupled to a Thermo Finnigan NEPTUNE MC-ICP-MS located at Oak Ridge National Laboratory (ORNL). The multicollector detector consists of an array of Faraday cups capable of simultaneously detecting up to seven isotopes. A secondary electron multiplier (SEM) is also included when more sensitive detection is required. The Faraday cup detector was used to measure both the ${ }^{235} U$ and ${ }^{238} U$ signal initially. If the ${ }^{235} \mathrm{U}$ signal fell below $1 \mathrm{mV}$ for a sample, measurement of ${ }^{235} \mathrm{U}$ was performed by the SEM. A detector integration interval of $131 \mathrm{~ms}$ was used throughout the study.

All measurements were performed in low resolution to maximize sensitivity. Faraday cup signals below a $1 \mathrm{mV}$ threshold were discarded for falling below the background. The 
minimum acceptable signal for SEM measurements was 250 counts per integration, while the maximum acceptable signal was $10^{5}$ counts per integration. The upper signal boundary was necessary as a result of detector nonlinearity at higher counting rates. Argon alone was used to supply all gas flows in this work. Tuning was performed daily for the MC-ICP-MS using solutions introduced with an Apex desolvating nebulizer.

\section{Materials}

Uranium oxide particulate samples were prepared from six certified reference materials (CRM) (U0002, U005-A, 129-A, U015, U020-A, and U030-A) provided by New Brunswick Laboratory (NBL) - United States Department of Energy. Slurry solutions containing $20 \mathrm{mg} \mathrm{mL}^{-1} \mathrm{U}$ in $20 \%$ collodion in ethanol were prepared for each CRM. Serial dilutions of 10x to 10,000x were performed on each standard solution. Samples for laser ablation were prepared by spotting $20 \mu \mathrm{L}$ of solution on a cotton Texwipe swipe. Swipes with nominal total $\mathrm{U}$ deposits of $40 \mu \mathrm{g}, 400 \mathrm{ng}$ and $40 \mathrm{ng}$ were produced for each CRM. Dissolution and analysis of samples from the $40 \mathrm{ng}$ series by solution nebulization ICP-MS showed a total U mass of 15.5 to $35.5 \mathrm{ng}$.

Environmental particulate samples were collected on Texwipes for analysis from areas known to feature $\mathrm{U}$ contamination. The $100 \mathrm{~mm}$ square Texwipes were quartered to fit the laser ablation cell. A drop of $20 \%$ collodion in ethanol was used to immobilize particles on the Texwipe to immobilize particles and establish similar sample conditions as the prepared uranium oxide standards. Samples were left to dry in a chemical hood prior to analysis. These samples were dissolved and analyzed by solution nebulization ICP-MS after the LA analysis was completed at ORNL. 
A solution series was prepared from certified reference materials from the Joint Research Centre's Institute for Reference Materials and Measurements (IRMM-183, -184, $-185,-186)$ and NBL (CRM U010). The solutions were used for mass bias corrections during the analysis. A standard solution of the appropriate isotopic ratio was measured both prior to and after ablation of a prepared swipe sample for these corrections.

\section{Results and Discussion}

\section{U Particulate Standard Samples}

For each level of prepared U-loading, a least-squares fit is constructed for the data collected across the entire CRM-series (Fig. 1 through 3). Each plot features the calculated 95\% prediction and confidence intervals for the data set. An expanded view is displayed

near the natural isotopic ratio for ${ }^{235} \mathrm{U} /{ }^{238} \mathrm{U}$, which is plotted as a comparison between the experimental values from this analysis and the literature value.

All data for the highest sample loading, $40 \mu \mathrm{g}$, in Fig. 1 were collected from ablations of the sample in a single spot with 100 laser shots. Uranium oxide particles for this sample were discernible using the optical camera system (Fig. 4), allowing discrete subjects to be chosen for each ablation. This was not possible with the lower U-loading of $400 \mathrm{ng}$ (Fig. 2) and $40 \mathrm{ng}$ (Fig. 3). The raster patterns were employed for these samples since no particles could be definitively identified.

Correlation coefficients for the least-squares fit of the data range from $\mathrm{R}=0.9987$ for the $400 \mathrm{ng}$ sample series to $\mathrm{R}=0.9997$ for the $40 \mu \mathrm{g}$ and $40 \mathrm{ng}$ sample series. The smallest confidence and prediction intervals are calculated for the $40 \mu \mathrm{g}$ sample series. A more accurate isotopic ratio for naturally abundant $U$ is calculated from the measured signals for 
the $40 \mu \mathrm{g}$ sample series as compared to the two lower sample loading series. While better accuracy and precision is expected for the samples featuring higher sample loadings, the poorer figures of merit found, especially for the $400 \mathrm{ng}$ sample series, are exacerbated by an additional analytical issue issue arising from the detectors used in the analysis.

In order to accommodate signals below the noise threshold of the Faraday cup array, it was sometimes necessary to use an SEM for measurement of the minor ${ }^{235} \mathrm{U}$ isotope. The SEM was needed more often as samples with lower mass loadings were analyzed. However the SEM was prone to saturation if too much uranium was introduced into the ICP during a given ablation, undercounting the signal for the minor isotope. This presents a challenge for samples like the $400 \mathrm{ng}$ set, where the mass loading requires the sensitivity provided by the SEM but makes detector saturation a possibility for each ablation. At lower sample loadings, such as the $40 \mathrm{ng}$ series, this concern is relieved as detector saturation becomes less probable.

Precision for all sample series suffered from spikes in the measured signal, as illustrated in Fig. 5. The extent of the effect of these signal spikes on the calculated isotope ratios varies with sample loading. For the $40 \mu \mathrm{g}$ loading of NBL CRM 129-A (Fig. 5a), little influence is apparent on the ${ }^{235} \mathrm{U} /{ }^{238} \mathrm{U}$ ratio. In contrast, the ${ }^{235} \mathrm{U} /{ }^{238} \mathrm{U}$ ratio for NBL $\mathrm{U} 015$ (Fig. 5b) changes throughout the duration of the signal transients. While this is the most extreme example of this effect and not typical for most samples, the cause of this behavior is an important consideration for this type of analysis.

Spikes on the signal transient have been found to result from the introduction of large particles into the plasma that are incompletely atomized by the ICP $[37,38]$. Large particles in this analysis most likely come from liberation of particulates near but not directly in the ablated spot on the sample. When the laser strikes the sample surface a shockwave is 
produced in the sample, which can eject particulates from the area around the ablation crater. Whereas material directly ablated is fragmented into smaller particles by the laser, particulates freed by the force of the shockwave are transported intact into the sample aerosol. The layer of collodion is used to minimize this effect but particles close enough to the ablation crater are likely liberated nonetheless.

These larger particles, unable to be fully atomized in the ICP, cause rapid increases in the atomic ion signal measured at the detector. This problem is magnified for detectors that are not designed to measure quickly changing signals, such as the Faraday cup array used in this study $[39,40]$. Mixed detector analyses involving SEMs and Faraday cups introduce further complications through different detector response timings. These detector issues result in cases like that presented in Fig. 5b, where spikes in each atomic ion signal do not track with each other and cause poor precision in the isotopic ratio.

Given the other advantages provided by using an ICP-MC-MS for isotopic analysis, changes in sample preparation can be considered to reduce rapid changes in ion signals to accommodate the detectors used for these instruments. Careful evaluation of the data can also address the detector issues described. Isotopic ratios can be calculated from sections in the signal transient free from large spikes and within the dynamic range of both detectors. With these considerations, fs-LA-ICP-MC-MS can serve not only as a capable technique for the rapid screening of samples but as a method for the determination of accurate and precise isotopic ratios. 


\section{Environmental Particulates}

Three environmental samples of particulates of variable composition collected on single swipes (X, Y and Z) were analyzed to establish the fitness of LA-ICP-MC-MS for the type of samples typically collected for isotopic analysis. Much like the prepared standard samples with low uranium oxide loadings, two of the three samples ( $\mathrm{X}$ and $\mathrm{Y}$ ) featured little visible material. Without distinct particulates to select, single spot ablations did not yield measurable signal for either sample. Ablating a larger area of the sample surface with a raster pattern (pattern B) produced measurable signal for both samples.

An example of the results provided by a raster analysis of sample $\mathrm{X}$ is presented in Fig. 6 and displays one of the advantages of LA for this work. As the sample is moved under the laser beam, distinct ${ }^{235} \mathrm{U} /{ }^{238} \mathrm{U}$ ratios are found at five separate locations. A similar isotope ratio profile is shown in Fig. 7 for sample Y, with three unique ratios detected across the sample. Tables 1 and 2 summarize the various ${ }^{235} \mathrm{U} /{ }^{238} \mathrm{U}$ ratios observed from samples $\mathrm{X}$ and $\mathrm{Y}$, respectively. Different ratios are found not only within the $2 \mathrm{~mm}$ square of the raster pattern but at other locations selected about the sample.

Information on the spatial distribution of different isotope ratios would not be possible if only wet chemical analysis were performed. Knowledge of the various ratios found in the particulate samples can supplement the results of a wet chemical analysis conducted when ablation analysis of the sample is completed. After ablation was completed, the samples were ashed, dissolved and analyzed by solution nebulization ICP-MC-MS. The ${ }^{235} \mathrm{U} /{ }^{238} \mathrm{U}$ ratio calculated from this wet chemical analysis is plotted in Fig. 8 for sample $\mathrm{X}$ and Fig. 9 for sample Y, overlaid with the average ratio from LA analysis on a histogram of the individual results from ablation of the samples. The ablation results reveal the various $U$ 
isotopic ratios that constitute the average value for the bulk sample. Since only a small area is examined by LA, the average for the ablation measurements does not correspond to the average from dissolution of the sample. The utility of LA for these types of samples lies in screening for samples of interest and providing a survey of the various isotope ratio values that contribute to the average measured ratio.

Unlike samples $X$ and $Y$, particulate material was clearly visible on sample Z. Single spot analysis was attempted on visualized particulates, but only one in ten attempts produced measurable U signal. Raster analysis with pattern A yielded measurable U signal for all ablations and again produced multiple isotopic compositions within the rastered area. Average ${ }^{235} \mathrm{U} /{ }^{238} \mathrm{U}$ ratios for each ablation analysis are reported in Table 3, with a histogram showing the isotopic ratio distribution for the whole dataset for sample $\mathrm{Z}$ in Fig. 10.

The failure of single spot ablation to produce measurable $U$ signals despite the abundance of visible particulates presents a limitation of trying to identify discrete particulates for analysis. Only a small portion of the material collected from a given area may contain the desired elements. Even though sample $\mathrm{Z}$ contained the most visible material, the total mass of $\mathrm{U}$ was found to be only $37.7 \mathrm{ng}$ by wet chemical analysis. This is less than sample $\mathrm{Y}$, found to contain a total mass of $\mathrm{U}$ of $60.4 \mathrm{ng}$, for which no particulate matter was discernable. Rather than perform many single spot ablations to achieve a positive result, the use of a raster pattern for LA can more efficiently investigate these environmental samples whether particulates are visible or not. 


\section{Conclusions}

The application of fs-LA-ICP-MC-MS to U-containing particulate samples provides a rapid and effective tool for the identification and isotopic analysis of samples of interest. Results from the examination of prepared uranium oxide particulate standards on Texwipes support the ability of this technique to produce both accurate and precise isotope ratios. For samples of a homogeneous isotopic composition, this technique can be used in lieu of wet chemistry methods to significantly reduce sample preparation and analysis time.

Examination of environmental particulate samples reveals how fs-LA-ICP-MC-MS can also complement wet chemical analysis. Rastering the laser across an area to ablate the sample enables calculation of the ${ }^{235} \mathrm{U} /{ }^{238} \mathrm{U}$ ratio at discrete positions. As observed in this study, environmental samples may not feature homogeneous isotopic compositions. Ablation preserves information on the spatial distribution of isotope ratios, which is lost when preparing the sample for wet chemical analysis. The average ${ }^{235} \mathrm{U} /{ }^{238} \mathrm{U}$ ratio determined by wet chemical methods can be more fully interpreted in light of the added information on the ratios present throughout the sample.

Further improvements can be made to the precision and accuracy of the method employed in this study. The precision enabled by using an ICP-MC-MS is vital to the effectiveness of this technique. The detectors used in these instruments, however, are not designed to handle rapidly changing signals, which were frequently encountered in this analysis as spikes on the signal transient. A likely source of the spikes is larger particles ejected from areas adjacent to the ablation crater. To accommodate the instrumentation, changes can be investigated in the preparation of the samples. Since collodion may not be effective in keeping particles nearby the ablation site immobilized, the efficacy of other 
substances in affixing the particulate matter to the Texwipe will be studied. Additional optimization of the detector combination used will also be carried out for different sample mass loadings to minimize detector saturation when employing an SEM alongside the Faraday cup array. Addressing these issues will increase the utility of fs-LA-ICP-MC-MS as a robust standalone technique for the isotopic analysis of particulate samples.

\section{Acknowledgements}

This research was supported by the U. S. Department of Energy, Office of Nuclear Nonproliferation (NA-22). The Ames Laboratory is operated for the U. S. Department of Energy by Iowa State University under Contract No. DE-AC02-07CH11358. Oak Ridge National Laboratory is operated for the U. S. Department of Energy by the University of Tennessee-Battelle.

\section{References}

[1] M.E. Kylander, D.J. Weiss, T.E. Jeffries, B. Kober, A. Dolgopolova, R. GarciaSanchez, B.J. Coles, A rapid and reliable method for $\mathrm{Pb}$ isotopic analysis of peat and lichens by laser ablation-quadrupole-inductively coupled plasma-mass spectrometry for biomonitoring and sample screening, Anal. Chim. Acta 582 (2007) 116-124.

[2] S. Steely, D. Amarasiriwardena, J. Jones, J. Yanez, A rapid approach for assessment of arsenic exposure by elemental analysis of single strand of hair using laser ablationinductively coupled plasma-mass spectrometry, Microchem. J. 86 (2007) 235-240.

[3] Z. Pedrero, Y. Madrid, C. Camara, E. Schram, J.B. Luten, I. Feldmann, L. Waentig, H. Hayen, N. Jakubowski, Screening of selenium containing proteins in the Trisbuffer soluble fraction of African catfish (Clarias gariepinus) fillets by laser ablationICP-MS after SDS-PAGE and electroblotting onto membranes, J. Anal. At. Spectrom. 24 (2009) 775-784.

[4] G.J. Baldwin, P.C. Thurston, B.S. Kamber, High-precision rare earth element, nickel, and chromium chemistry of chert microbands pre-screened with in-situ analysis, Chem. Geol. 285 (2011) 133-143. 
[5] J. Messerly, N. Saetveit, S. Bajic, D. Baldwin, S. Houk, Analysis of Enriched and Depleted Uranium Oxide Powders by LA-ICP-MS - Final Report, Ames Laboratory Report IS-5174 (2005).

[6] X.Z. Zhang, F. Esaka, K.T. Esaka, M. Magara, S. Sakurai, S. Usuda, K. Watanabe, Applications of Inductively Coupled Plasma Mass Spectrometry to the determination of uranium isotope ratios in individual particles for nuclear safeguards, Spectrochim. Acta Part B 62 (2007) 1130-1134.

[7] J.S. Becker, H. Sela, J. Dobrowolska, M. Zoriy, J.S. Becker, Recent applications on isotope ratio measurements by ICP-MS and LA-ICP-MS on biological samples and single particles, Int. J. Mass. Spectrom. 270 (2008) 1-7.

[8] M. Aramendia, M. Resano, F. Vanhaecke, Isotope ratio determination by laser ablation-single collector-inductively coupled plasma-mass spectrometry: General capabilities and possibilities for improvement, J. Anal. At. Spectrom. 25 (2010) 390404.

[9] C.M. Fisher, H.P. Longerich, S.E. Jackson, J.M. Hanchar, Data acquistion and calculation of $\mathrm{U}-\mathrm{Pb}$ isotopic analyses using laser ablation (single collector) inductively coupled plasma mass spectrometry, J. Anal. At. Spectrom. 25 (2010) 1905-1920.

[10] R. Mertz-Kraus, K.P. Jochum, W.D. Sharp, B. Stoll, U. Weis, M.O. Andreae, In situ 230Th-232Th-234U-238U analysis of silicate glasses and carbonates using laser ablation single-collector sector-field ICP-MS, J. Anal. At. Spectrom. 25 (2010) 18951904.

[11] L.A. Solari, A. Gomez-Tuena, J.P. Bernal, O. Perez-Arvizu, M. Tanner, U-Pb zircon geochronology with an integrated LA-ICP-MS microanalytical workstation:

achievements in precision and accuracy, Geostand. Geoanal. Res. 34 (2010) 5-18.

[12] C. Latkoczy, Y. Mueller, P. Schmutz, D. Günther, Quantitative element mapping of magnesium alloys by laser ablation ICP-MS and EPMA, Appl. Surf. Sci. 252 (2005) 127-132.

[13] J.S. Becker, J. Dobrowolska, M. Zoriy, A. Matusch, Imaging of uranium on rat brain sections using laser ablation inductively coupled plasma mass spectrometry: a new tool for the study if critical substructures affined to heavy metals in tissues, Rapid Commun. Mass Sp. 22 (2008) 2768-2772.

[14] K. Novotny, J. Kaiser, M. Galiova, V. Konecna, J. Novotny, R. Malina, M. Liska, V. Kanicky, V. Otruba, Mapping of different structures on large area of granite sample using laser-ablation based analytical techniques, an exploratory study, Spectrochim. Acta Part B 63 (2008) 1139-1144.

[15] V.S. Selih, J.T. van Elteren, Quantitative multi-element mapping of ancient glass using a simple and robust LA-ICP-MS rastering procedure in combination with image analysis, Anal. Bioanal. Chem. In Press (2011).

[16] B. Rusk, A. Koenig, H. Lowers, Visualizing trace element distribution in quartz using cathodoluminescence, electron microprobe, and laser ablation-inductively coupled plasma-mass spectrometry, Am. Mineral. 96 (2011) 703-708. 
[17] T. Prohaska, S. Hann, C. Latkoczy, G. Stingeder, Determination of rare earth elements $\mathrm{U}$ and $\mathrm{Th}$ in envrionmental samples by inductively coupled plasma double focusing sectorfield mass spectrometry (ICP-SMS), J. Anal. At. Spectrom. 14 (1999) $1-8$.

[18] S. Uchida, R. Garcia-Tenorio, K. Tagami, M. Garcia-Leon, Determination of U isotopic ratios in environmental samples by ICP-MS, J. Anal. At. Spectrom. 15 (2000) 889-892.

[19] R.D. Evans, H. Hintelmanna, P.J. Dillon, Measurement of high precision isotope ratios for mercury from coals using transient signals, J. Anal. At. Spectrom. 16 (2001) 1064-1069.

[20] S. D'Ilio, N. Violante, O. Senofonte, C. Majorani, F. Petrucci, Determination of depleated uranium in human hair by quadrupole inductively coupled plasma mass spectrometry: method development and validation, Anal. Methods 2 (2010) 11841190.

[21] A.L. Gray, Solid sample introduction by laser ablation for inductively coupled plasma source mass spectrometry, Analyst 110 (1985) 551-556.

[22] D. Günther, B. Hattendorf, Solid sample analysis using laser ablation inductively coupled plasma mass spectrometry, TrAC-Trend. Anal. Chem. 24 (2005) 255-265.

[23] J. González, S.H. Dundas, C. Liu, X. Mao, R.E. Russo, UV-femtosecond and nanosecond laser ablation-ICP-MS: internal and external repeatability, J. Anal. Atom. Spectrom. 21 (2006) 778-784.

[24] N.J. Saetveit, S.J. Bajic, D.P. Baldwin, R.S. Houk, Influence of particle size on fractionation with nanosecond and femtosecond laser ablation in brass by online differential mobility analysis and inductively coupled plasma mass spectrometry, J. Anal. At. Spectrom. 23 (2008) 54-61.

[25] D.C. Perdian, S.J. Bajic, D.P. Baldwin, R.S. Houk, Time-resolved studies of particle effects in laser ablation inductively coupled plasma-mass spectrometry. Part 1. Investigation of nanosecond and femtosecond pulse width lasers and devices for particle size selection, J. Anal. At. Spectrom. 23 (2008) 325-335.

[26] R.E. Russo, X. Mao, J.J. González, S.S. Mao, Femtosecond laser ablation ICP-MS, J. Anal. At. Spectrom. 17 (2002) 1072-1075.

[27] V. Možná, J. Pisonero, M. Holá, V. Kanický, D. Günther, Quantitative analysis of Febased samples using ultraviolet nanosecond and femtosecond laser ablation-ICP-MS, J. Anal. At. Spectrom. 21 (2006) 1194-1201.

[28] C. Liu, X. Mao, S.S. Mao, X. Zeng, R. Greif, R.E. Russo, Nanosecond and Femtosecond Laser Ablation of Brass: Particulate and ICP-MS Measurements, Anal. Chem. 76 (2004) 379-383.

[29] Z. Wang, B. Hattendorf, D. Günther, Vaporization and ionization of laser ablation generated aerosols in an inductively coupled plasma mass spectrometer - implications from ion distribution maps, J. Anal. At. Spectrom. 21 (2006) 1143-1151.

[30] F. Poitrasson, X. Mao, S.S. Mao, R. Freydier, R.E. Russo, Comparison of Ultraviolet Femtosecond and Nanosecond Laser Ablation Inductively Coupled Plasma Mass Spectrometry Analysis in Glass, Monazite, and Zircon, Anal. Chem. 75 (2003) 61846190. 
[31] J. Koch, D. Günther, Femtosecond laser ablation inductively coupled plasma mass spectrometry: achievements and remaining problems, Anal. Bioanal. Chem. 387 (2007) 149-153.

[32] C. Ebert, D.S. Zamzow, E. McBay, D.A. Bostick, S.J. Bajic, D.P. Baldwin, R.S. Houk, Elemental and Isotopic Analysis of Uranium Oxide and NIST Glass Standards by Femtosecond-LA-ICP-MIC-MS, Ames Laboratory Report IS-5194 (2009).

[33] C.H. Stirling, D.-C. Lee, J.N. Christensen, A.N. Halliday, High-precision in situ 238U-234U-230Th isotopic analysis using laser ablation multiple-collector ICPMS, Geochim. Cosmochim. Ac. 64 (2000) 3737-3750.

[34] T. Walczyk, TIMS versus multicollector-ICP-MS: coexistence or struggle for survival?, Anal. Bioanal. Chem. 378 (2004) 229-231.

[35] S. Johnston, G. Gehrels, V. Valencia, J. Ruiz, Small-volume U-Pb zircon geochronology by laser ablation-multicollector-ICP-MS, Chem. Geol. 259 (2009) 218-229.

[36] R. Santamaria-Fernandez, Precise and traceable carbon isotope ratio measurements by multicollector ICP-MS: what next?, Anal. Bioanal. Chem. 397 (2010) 973-978.

[37] R.S. Houk, R.K. Winge, C. Xiaoshan, High Speed Photographic Study of Wet Droplets and Solid Particles in the Inductively Coupled Plasma, J. Anal. At. Spectrom. 12 (1997) 1139-1148.

[38] M. Guillong, D. Günther, Effect of particle size distribution on ICP-induced elemental fractionation in laser ablation-inductively coupled plasma-mass spectrometry, J. Anal. At. Spectrom. 17 (2002) 831-837.

[39] R. Thomas, A Beginner's Guide to ICP-MS: Part X - Detectors, Spectroscopy 17 (2004) 34-39.

[40] M. Tanner, D. Günther, Short transient signals, a challenge for inductively coupled plasma mass spectrometry, a review, Anal. Chim. Acta 633 (2009) 19-28. 

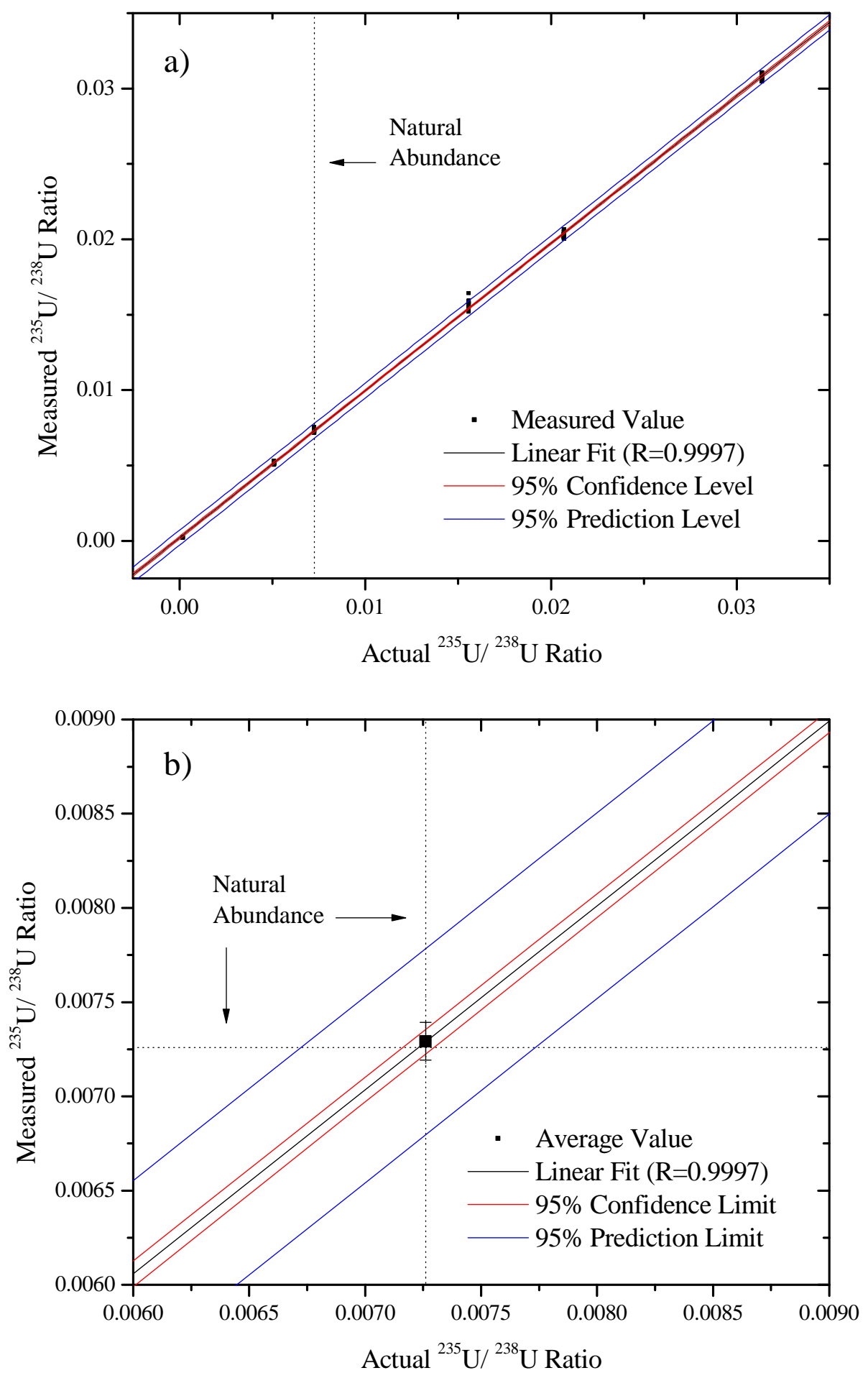

Fig. 1. (a) Least-squares fit of measured ${ }^{235} \mathrm{U} /{ }^{238} \mathrm{U}$ ratios for $40 \mu \mathrm{g}$ sample uranium oxide sample series (b) Expanded view on the measured ${ }^{235} \mathrm{U} /{ }^{238} \mathrm{U}$ ratio for the natural abundance sample 

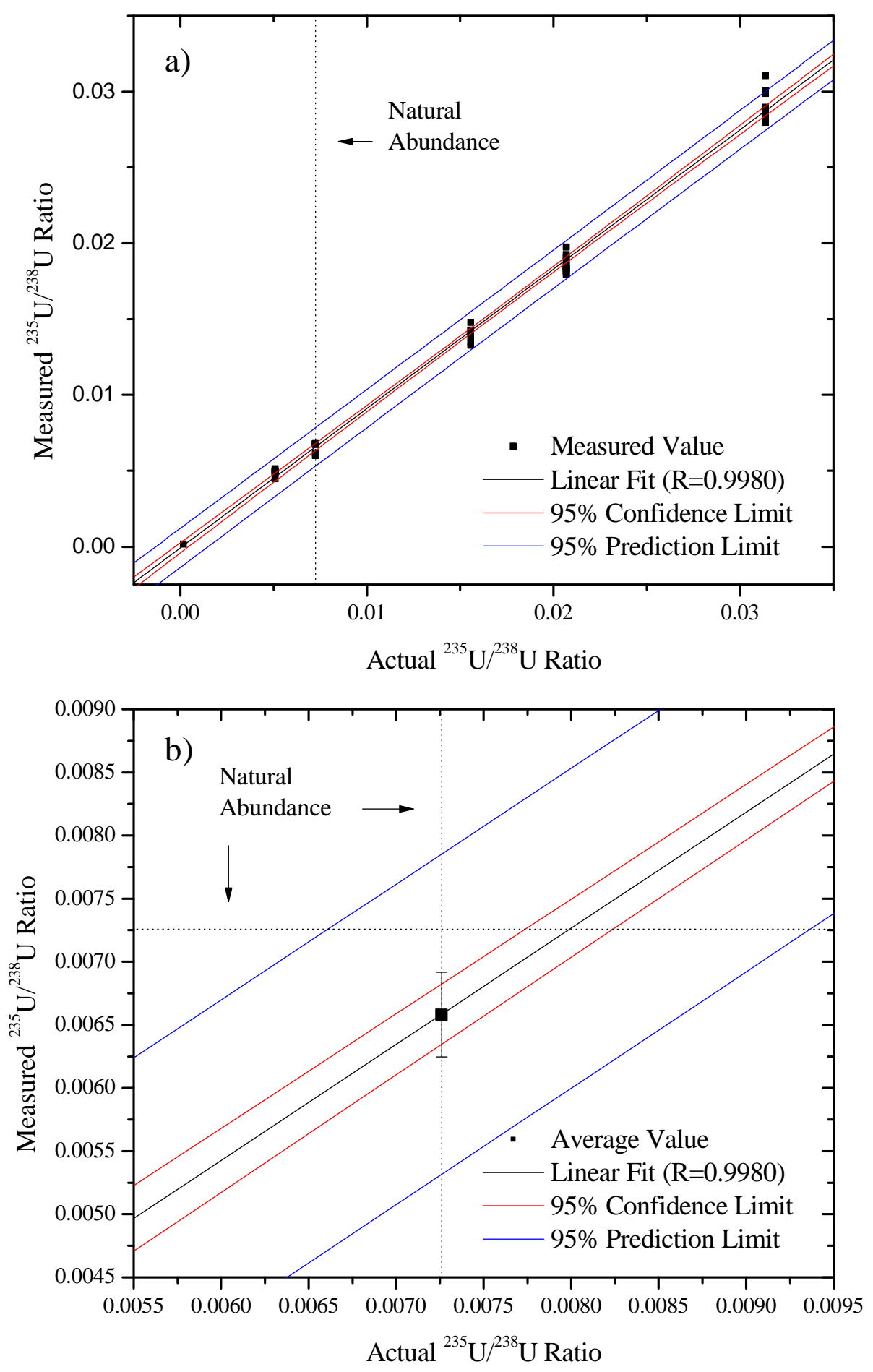

Fig. 2. (a) Least-squares fit of measured ${ }^{235} U{ }^{238} U$ ratios for $400 \mathrm{ng}$ sample uranium oxide sample series (b) Expanded view on the measured ${ }^{235} \mathrm{U} /{ }^{238} \mathrm{U}$ ratio for the natural abundance sample 

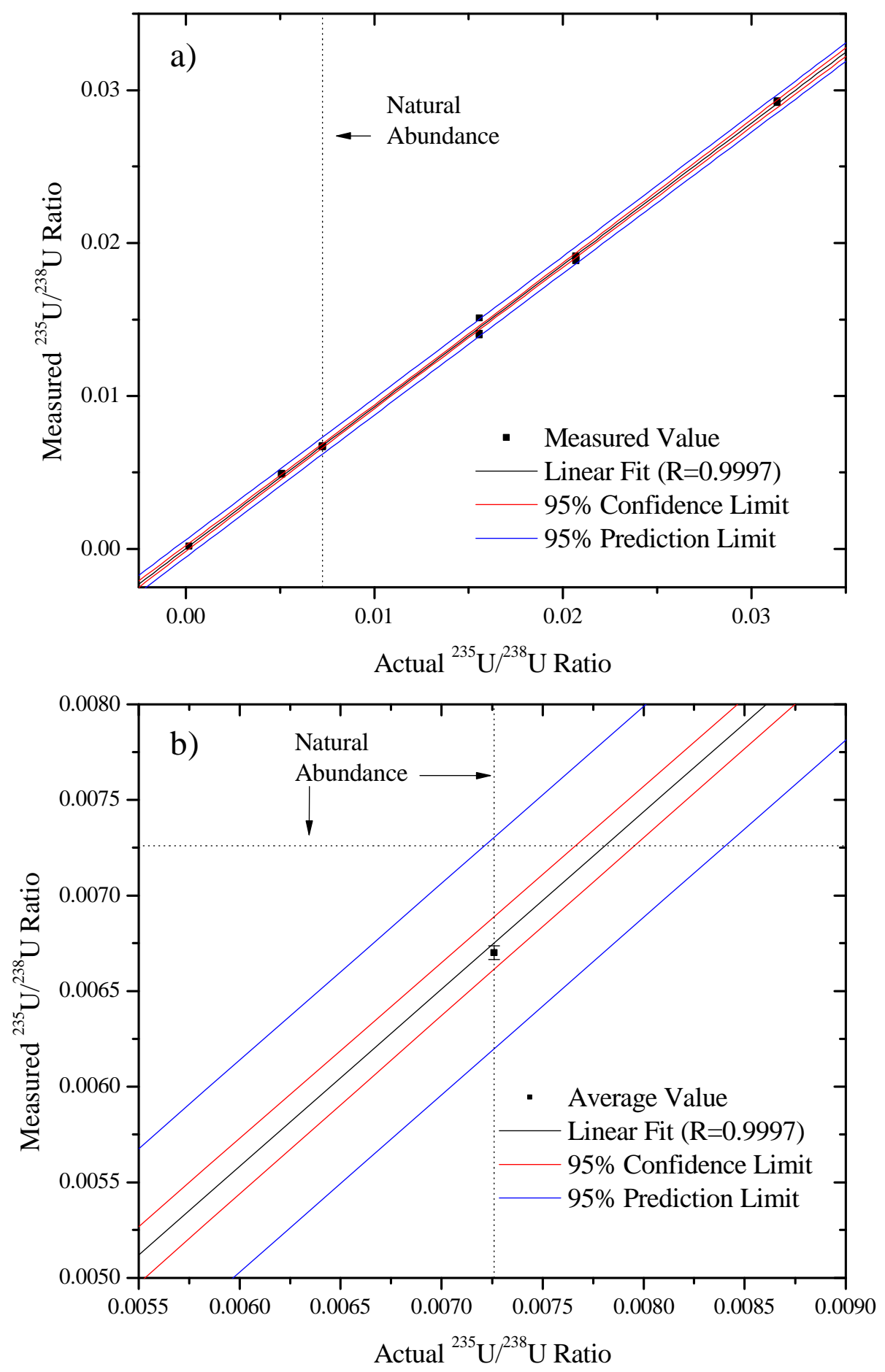

Fig. 3. (a) Least-squares fit of measured ${ }^{235} U /{ }^{238} U$ ratios for $40 \mathrm{ng}$ sample uranium oxide sample series (b) Expanded view on the measured ${ }^{235} \mathrm{U} /{ }^{238} \mathrm{U}$ ratio for the natural abundance sample 


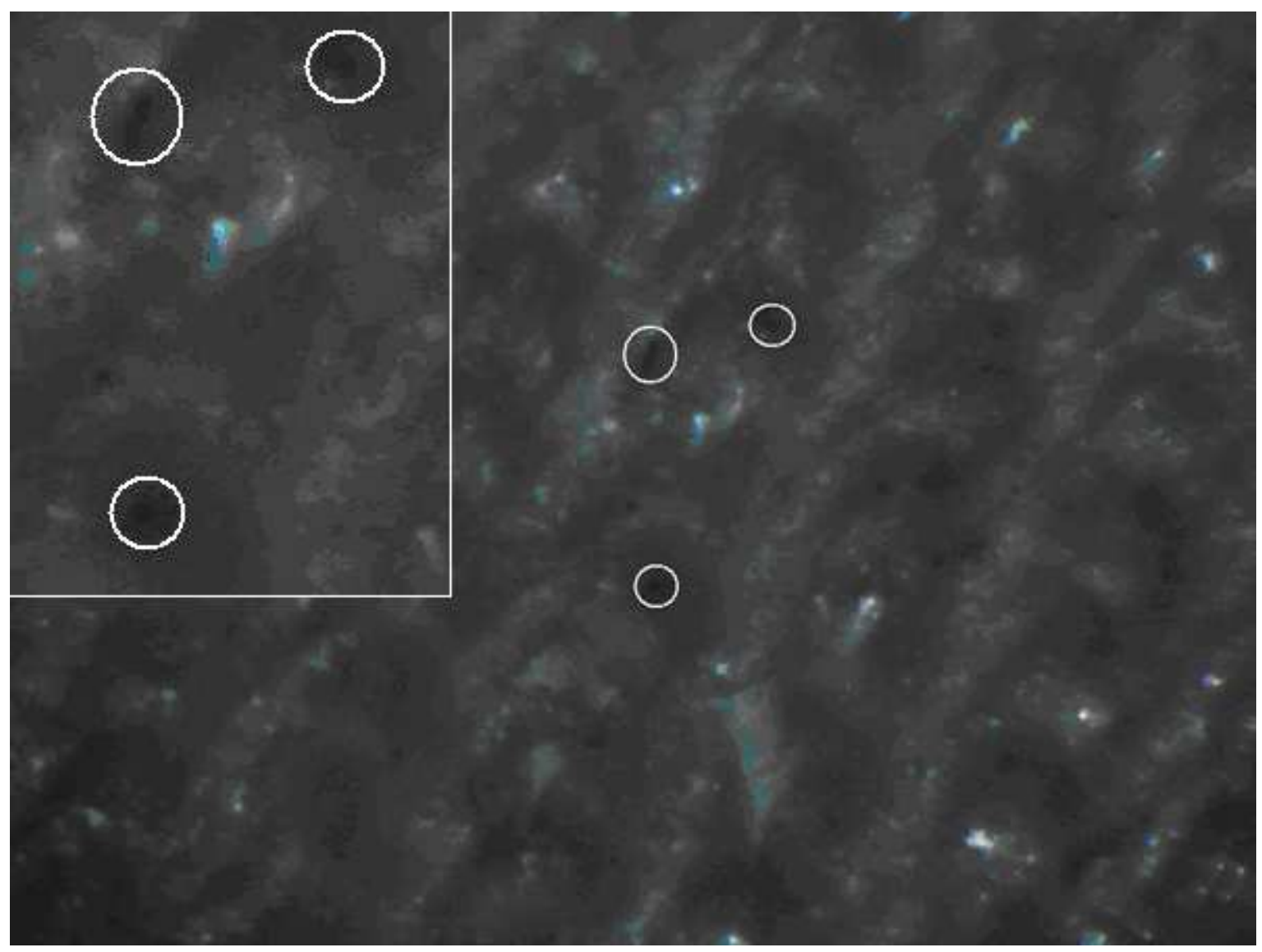

Fig. 4. Image from fs-LA homemade camera system of U particulates (indicated by white circles). Individual particles are able to be selected for ablation based on this visualization. (Inset: Expanded view created from original camera image) 

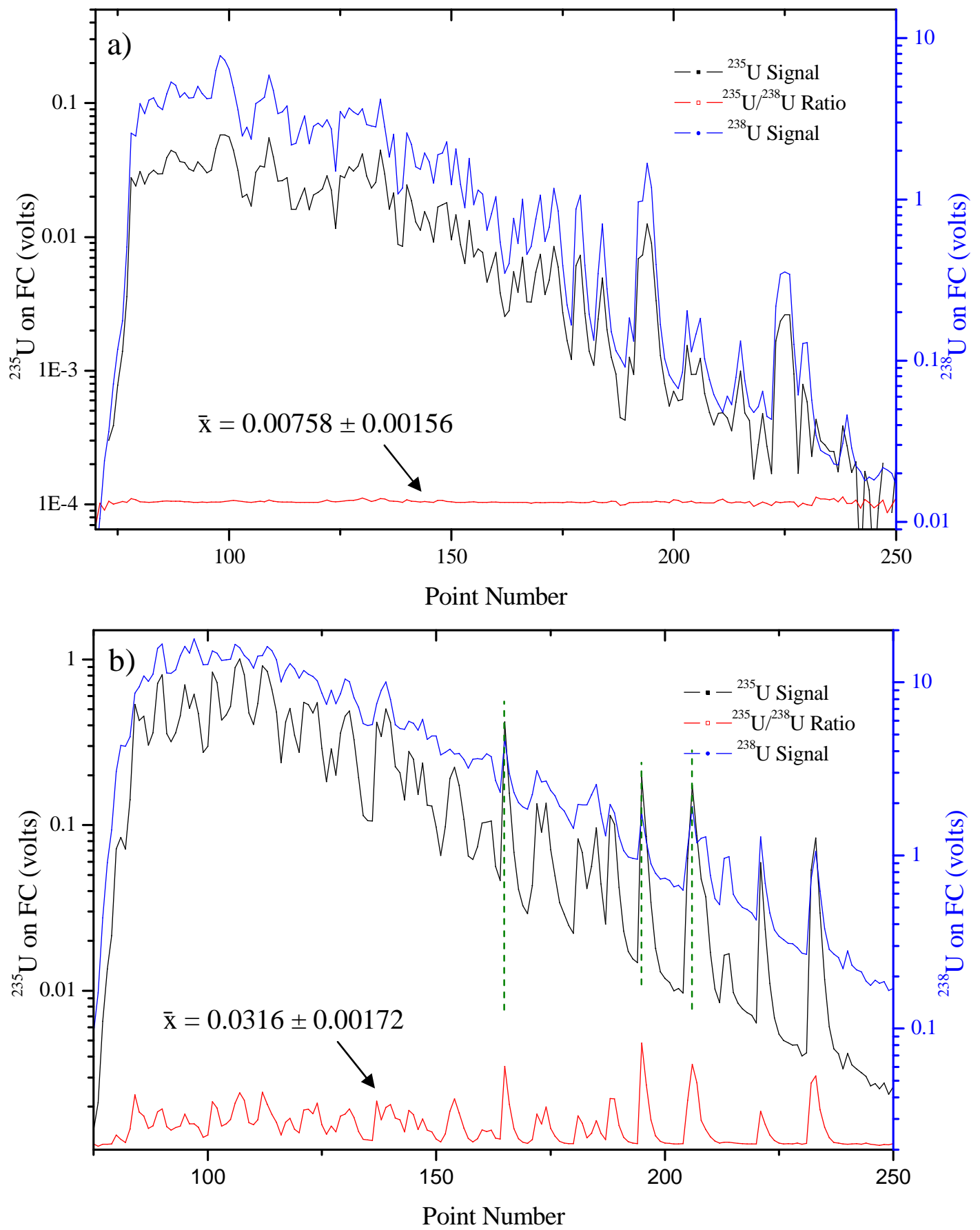

Fig. 5. Signal transients and calculated ratios (red) for (a) NBL CRM 129-A and (b) NBL U015 showing the effect of signal spikes on isotope ratios. The ${ }^{235} \mathrm{U}$ signal (black) has been multiplied by an appropriate factor to enable plotting on the same scale as the ${ }^{238} \mathrm{U}$ signal (blue). 


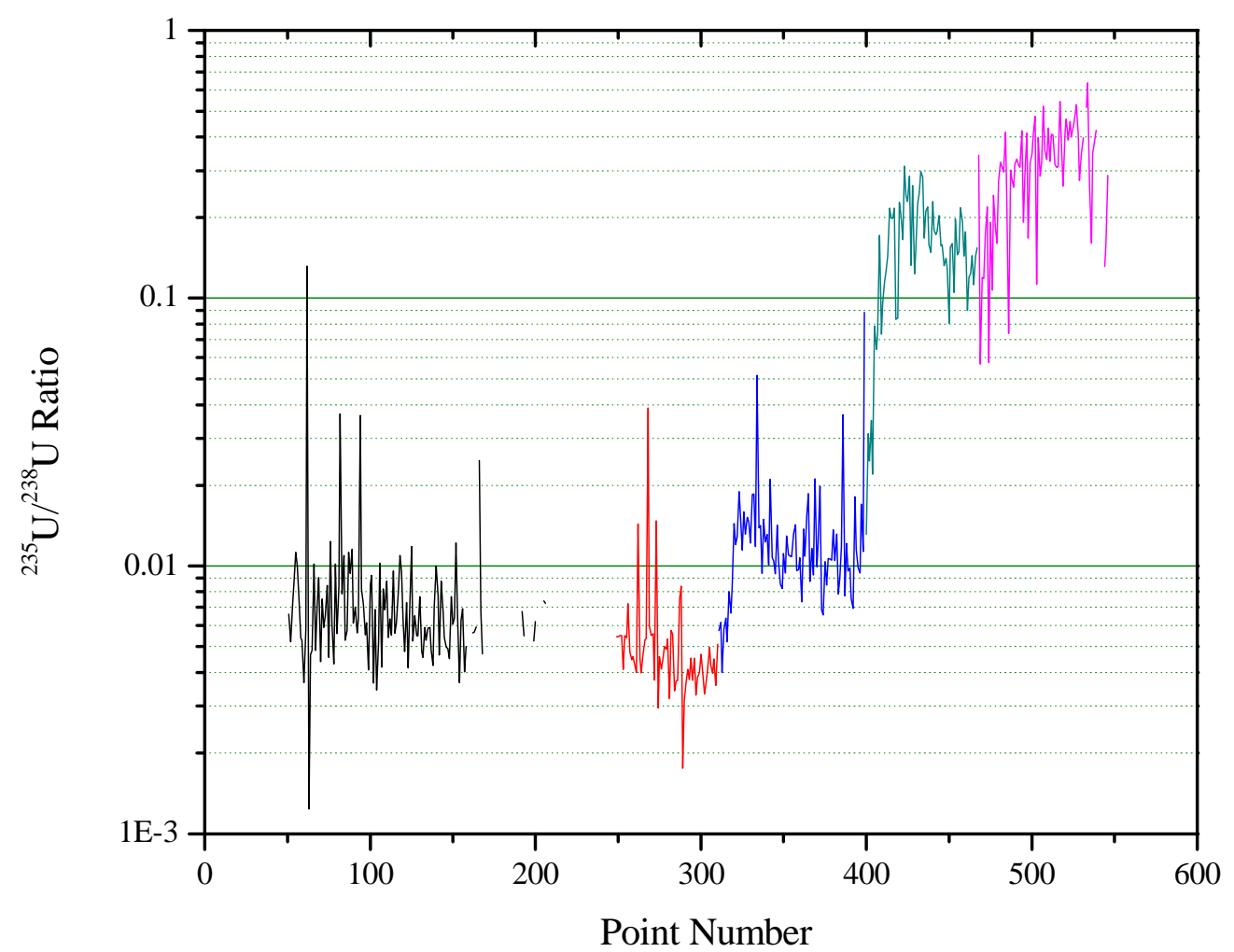

Fig. $6 .{ }^{235} \mathrm{U} /{ }^{238} \mathrm{U}$ ratio calculated at each point during a raster (pattern $\mathrm{B}$ ) across a $2 \mathrm{~mm}$ square area of sample $\mathrm{X}$. 


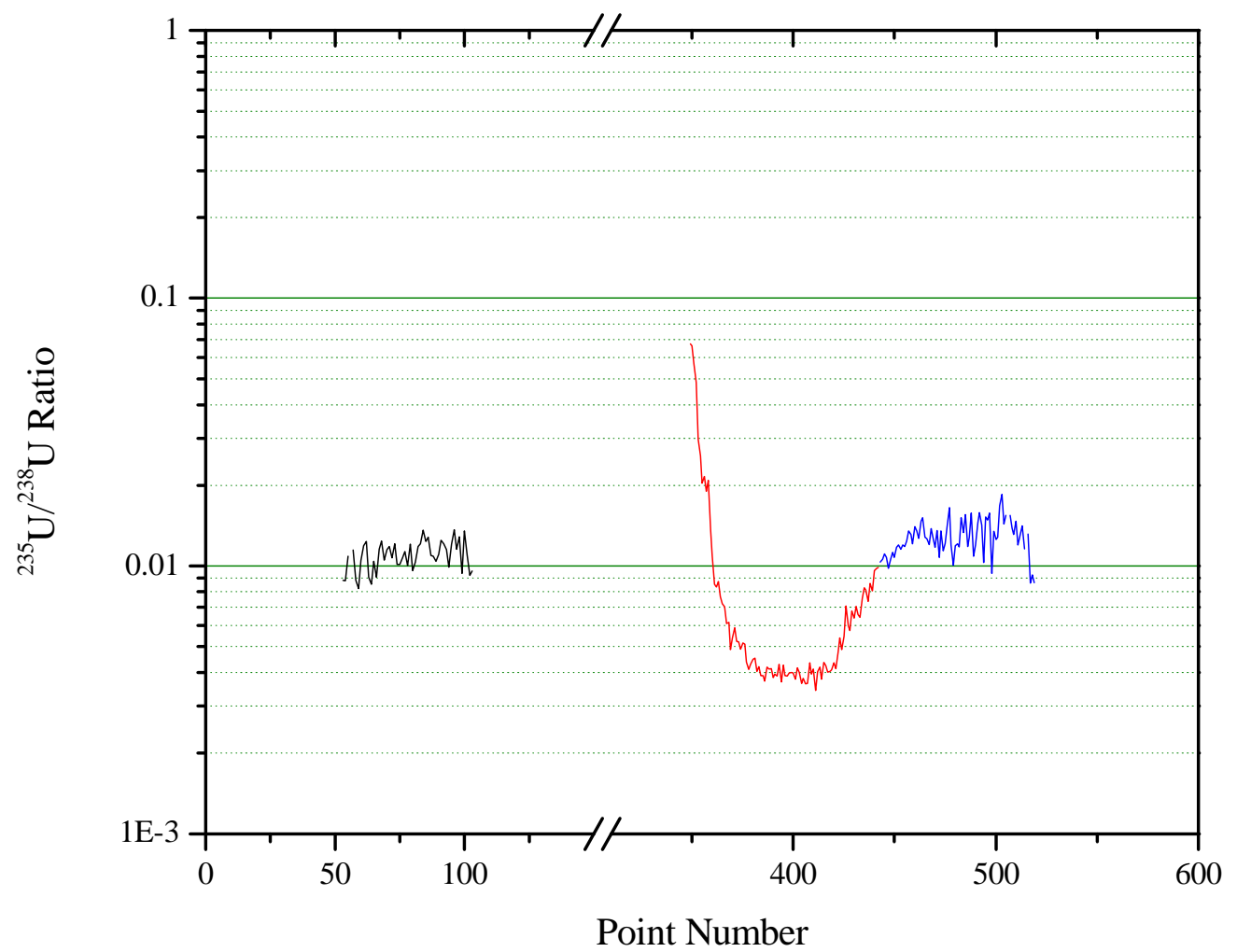

Fig. 7. ${ }^{235} \mathrm{U} /{ }^{238} \mathrm{U}$ ratio calculated at each point during a raster (pattern $\mathrm{B}$ ) across a $2 \mathrm{~mm}$ square area of sample $\mathrm{X}$. 


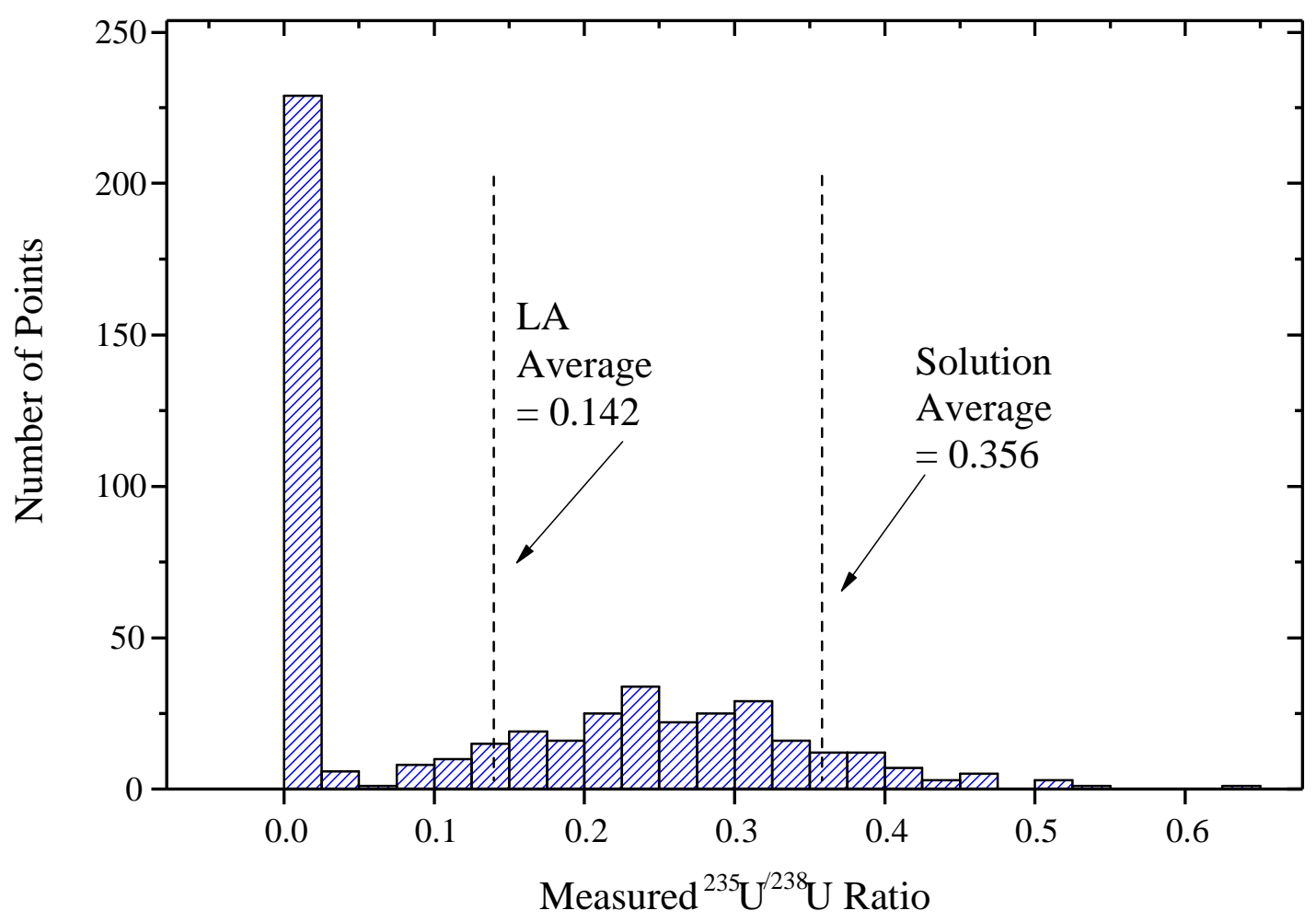

Fig. 8. Histogram of the measured ${ }^{235} \mathrm{U} /{ }^{238} \mathrm{U}$ ratio for environmental sample $\mathrm{X}$. Average ratios from ablation and wet chemical analysis are reported. 


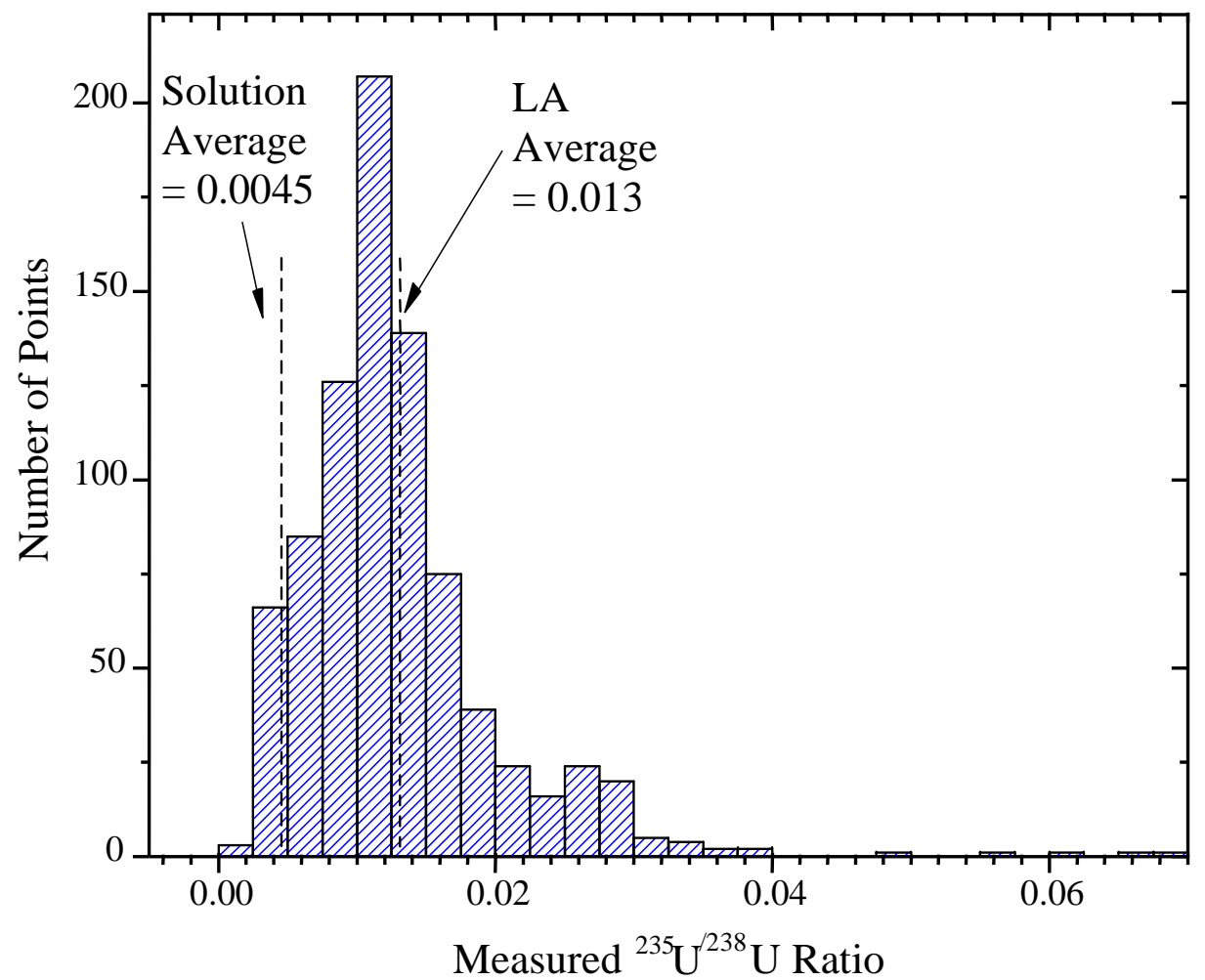

Fig. 9. Histogram of the measured ${ }^{235} \mathrm{U} /{ }^{238} \mathrm{U}$ ratio for environmental sample Y. Average ratios from ablation and wet chemical analysis are reported. 


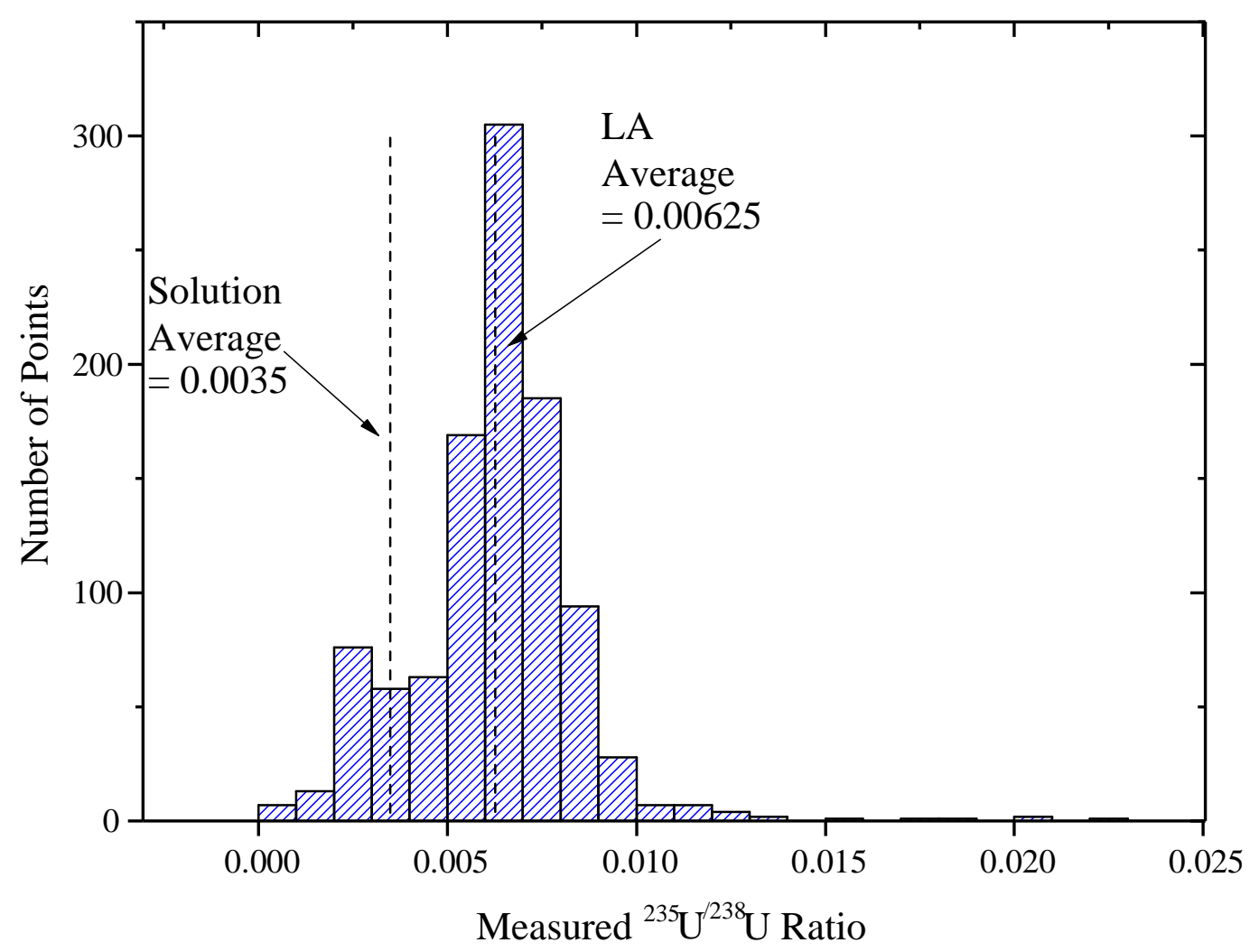

Fig. 10. Histogram of the measured ${ }^{235} \mathrm{U} /{ }^{238} \mathrm{U}$ ratio for environmental sample Y. Average ratios from ablation and wet chemical analysis are reported. 
Table 1. Measured uranium isotope ratios in different spatial locations on sample $\mathrm{X}^{\mathrm{a}}$

\begin{tabular}{|c|c|c|}
\hline Location & ${ }^{235} \mathrm{U} /{ }^{238} \mathrm{U}$ & StDev \\
\hline 1 & 0.256 & 0.087 \\
\hline 2 & 0.262 & 0.074 \\
\hline 3 & 0.337 & 0.033 \\
\hline $4^{\dagger}$ & 0.008 & 0.013 \\
& 0.005 & 0.005 \\
& 0.013 & 0.006 \\
& 0.180 & 0.056 \\
& 0.382 & 0.100 \\
\hline
\end{tabular}

Raster analysis performed at all locations.

${ }^{\dagger}$ Indicates an area where more than one distinct isotope ratio region was detected.

${ }^{\mathrm{a}}$ Wet chemical average ${ }^{235} \mathrm{U} /{ }^{238} \mathrm{U}$ ratio for this sample: 0.356 
Table 2. Measured uranium isotope ratios in different spatial locations on sample $\mathrm{Y}^{\mathrm{b}}$

\begin{tabular}{|c|c|c|}
\hline Location & ${ }^{235} \mathrm{U}^{238} \mathrm{U}$ & StDev \\
\hline 1 & 0.0053 & 0.00102 \\
\hline $2^{\dagger}$ & 0.02842 & 0.01036 \\
& 0.01313 & 0.00626 \\
& 0.0279 & 0.0035 \\
\hline 3 & 0.00933 & 0.00322 \\
\hline 4 & 0.01373 & 0.00314 \\
\hline $5^{\dagger}$ & 0.01059 & 0.00318 \\
& 0.01424 & 0.00404 \\
& 0.00845 & 0.00111 \\
& 0.01887 & 0.00373 \\
\hline 6 & 0.0114 & 0.00492 \\
\hline $7^{\dagger}$ & 0.01129 & 0.00136 \\
& 0.01069 & 0.00949 \\
\hline
\end{tabular}

Raster analysis performed at all locations.

${ }^{\dagger}$ Indicates an area where more than one distinct isotope ratio region was detected.

${ }^{b}$ Wet chemical average ${ }^{235} \mathrm{U} /{ }^{238} \mathrm{U}$ ratio for this sample: 0.00453 
Table 3. Measured uranium isotope ratios in different spatial locations on sample $Z^{\mathrm{c}}$

\begin{tabular}{|c|c|c|}
\hline Location & ${ }^{235} \mathrm{U} /{ }^{238} \mathrm{U}$ & StDev \\
\hline $1^{*}$ & 0.00652 & 0.00212 \\
\hline $2^{\dagger}$ & 0.00733 & 0.00114 \\
& 0.00293 & 0.00044 \\
& 0.00637 & 0.0011 \\
\hline 3 & 0.00662 & 0.00239 \\
\hline 4 & 0.00696 & 0.00189 \\
\hline 5 & 0.00686 & 0.00142 \\
\hline $6^{\dagger}$ & 0.00695 & 0.00164 \\
& 0.0023 & 0.00074 \\
& 0.00351 & 0.0011 \\
& 0.00413 & 0.00123 \\
& 0.00575 & 0.00092 \\
\hline
\end{tabular}

Indicates single-spot analysis. All other locations were subject to raster analysis.

${ }^{\dagger}$ Indicates an area where more than one distinct isotope ratio region was detected.

${ }^{c}$ Wet chemical average ${ }^{235} \mathrm{U} /{ }^{238} \mathrm{U}$ ratio for this sample: 0.0035 


\section{Chapter 5. General Conclusions}

Advances in LA-ICP-MS, be it the expansion of the technique to new types of analyses or simply improved analytical performance for current analyses, are increasingly possible as our understanding of the ablation, atomization and extraction processes unique to this analytical method grows. The work discussed here is another contribution to this body of knowledge, addressing both fundamental aspects of polyatomic ion interferences in LAICP-MS and sampling strategies for an atypical sample class. It is hoped that some of the reported findings inspire additional investigations and innovations for the improvement of LA-ICP-MS as a versatile and reliable technique for trace elemental analysis.

Compared with solution nebulization ICP-MS, the ratio of $\mathrm{MO}^{+} / \mathrm{M}^{+}$ions is greatly reduced under the conditions found during LA-ICP-MS. Further moderate reductions in $\mathrm{MO}^{+}$ion interferences can be achieved by adding defined flow rates of $\mathrm{He}$ and $\mathrm{N}_{2}$ to the center channel of the Ar plasma. The physical effect of addition of these two gases to the ICP could be further revealed by visual studies of the plasma. Movies of an Ar-only and a mixed-gas plasma during introduction of visible light emitting ablated material could be compared to determine what alterations, if any, occur in the position of the ionization and atomization zones relative to the sampling interface.

Another possible source of polyatomic interferences, $\mathrm{MAr}^{+}$ions, is found to be much less abundant in the mass spectrum than theoretically predicted based on plasma conditions. It is hypothesized that collisional dissociation of the ions may be supported by a small plasma potential produced by incomplete shielding of the ICP. An analysis of the kinetic energy distribution of ions entering the mass spectrometer could assist in determining the 
existence of and energy imparted by such a potential. Depending on the size of the potential, other weakly-bound polyatomic ions may be beneficially dissociated during ion extraction.

The scope of samples suitable for analysis by LA-ICP-MS need not be limited to bulk solids. Elemental analysis can be conducted for particulate matter, including environmental particulates and nanoparticles, on a single particle basis. Particle selectivity can be enhanced with appropriate sample preparation to immobilize the particulate material. Additional study could identify the optimum preparation method by investigating both different particle immobilizers and schemes for their application. This could possibly minimize the occurrence of spikes on the signal transient and improve the precision of the technique. New experiments incorporating these observations and proposed research regarding polyatomic ion interferences and sampling methods may together prove valuable to the continued growth in use of LA-ICP-MS for precise and accurate trace elemental analysis. 


\section{Acknowledgements}

This research was performed at Ames Laboratory under Contract No. DEAC0207CH11358. The work in Chapter 2 was supported by the National Science Foundation (Award No. CHE-0309381) through the Institute for Physical Research and Technology at ISU. The work presented in Chapters 3 and 4 was supported by the U. S. Department of Energy, Office of Nuclear Nonproliferation (NA-22).

I am deeply indebted to numerous people for their support during my time as a graduate student at ISU. Foremost, I give thanks to Dr. R. S. Houk for his instruction and direction throughout my research. I would also like to acknowledge Stan Bajic, Dan Zamzow and David Baldwin for being ever-ready with assistance during the fs-LA-ICP-MS experiments. I certainly would not have completed this work without the friendship and help of my fellow Houk group members, both past and present. I consider myself very lucky to have shared the same dusty tiles with such great people. Thanks are also due the collaborators with whom I had so many productive interactions. Finally, I thank my friends and family for their love, for their encouragement and for the free lodging down the stretch run of my dissertation. 


\begin{abstract}
Appendix A.
Measurement Of Gas Kinetic Temperatures

For Polyatomic Ions In Inductively Coupled Plasma-

Mass Spectrometry: Validation And Refinements

Sally M. McIntyre, Jill Wisnewski Ferguson, Travis M. Witte and R.S. Houk
\end{abstract}

\author{
Ames Laboratory U.S. Department of Energy \\ Department of Chemistry \\ Iowa State University, Ames, IA 50011, USA
}

A paper published in Spectrochimica Acta, Part B, 2011, 66, 581-587. 


\begin{abstract}
The general method of comparing measured ion ratios to calculated ion ratios to determine a gas kinetic temperature $\left(\mathrm{T}_{\mathrm{gas}}\right)$ is reviewed. Various mathematical refinements to the calculated partition functions are examined for their effect on the determined $\mathrm{T}_{\text {gas }}$. It is found that (a) excited electronic states should be included for $\mathrm{ArO}^{+}$, neutral $\mathrm{NO}$, and $\mathrm{O}_{2}$; (b) a $10 \%$ error in solvent load, sample gas flow rate, vibrational constant $(\omega)$, rotational constant (B) or measured ion ratio produces only a 1 to $3 \%$ error in $\mathrm{T}_{\text {gas }}$; (c) a $10 \%$ error in dissociation energy $\left(\mathrm{D}_{0}\right)$ creates nearly a $10 \%$ error in $\mathrm{T}_{\text {gas }}$; and $(\mathrm{d})$ high temperature corrections to the partition functions produce minimal change and can generally be neglected.
\end{abstract}

\title{
1. Introduction
}

Inductively coupled plasma-mass spectrometry (ICP-MS) is one of the major elemental analysis techniques used today. An ICP-MS instrument works by atomizing and ionizing aerosols or gases that represent a sample of interest. Unfortunately, even at 5000 to $6000 \mathrm{~K}$, not all of the sample is converted to single atoms [1,2]. Statistical thermodynamics calculations show that a small fraction of polyatomic ions will survive the plasma, even those with weak bonds like $\mathrm{ArO}^{+}$and $\mathrm{Ar}_{2}^{+}\left(\mathrm{D}_{0}=0.312\right.$ and $\left.1.2 \mathrm{eV}\right)[3,4]$.

Polyatomic ions that interfere with elemental signals at the same nominal mass-tocharge $(\mathrm{m} / \mathrm{z})$ values lead to incorrect analyses and poorer limits of detection. Particular problems arise from ions containing $\mathrm{Ar}, \mathrm{O}, \mathrm{H}$ and $\mathrm{N}$ atoms due to the great abundance of those elements in the plasma, sample and/or solvent. Many studies have investigated the 
effect of various plasma and instrument conditions on polyatomic ion signals [5]. Methods such as collision cells [6,7], high resolution instruments [8], cool plasmas $[4,9,10]$, and solvent removal [11,12], have been developed to attenuate their effects. While these techniques have been very successful, a better fundamental understanding of the origin and behavior of polyatomic ions could lead to new types of ICP-MS instruments that intrinsically minimize the formation of these interfering ions. Existing techniques would then be even more effective resulting in lower limits of detection, better accuracy, higher throughput and lower costs.

Polyatomic ions do not only arise in the plasma, they could also be formed during the extraction process. The extent of formation of such "extra" polyatomic ions has been an objective of several studies. In 1988 Douglas and French applied a hemispherical sink model to the flow of plasma particles through the sampler cone of an ICP-MS. They calculated that an analyte atom would collide with argon atoms at most 250 times during the flow from Mach 0.7 (0.1 $\mathrm{mm}$ downstream from the sampler tip) to the skimmer tip [13]. Collisions between analyte atoms and atoms of oxygen and hydrogen from the sample solvent would be two to three orders of magnitude less frequent. They concluded that there were not enough collisions to produce appreciable additional amounts of metal oxide ions $\left(\mathrm{MO}^{+}\right)$after the Mach 0.7 position.

In 1994, Nonose et al. compared measured polyatomic ion signals to theoretical number densities calculated from spectroscopic data [4]. This allowed them to divide the polyatomic ions studied into two groups. $\mathrm{ArX}^{+}$species appeared to reach dissociation equilibrium in the interface. $\mathrm{NO}^{+}, \mathrm{O}_{2}{ }^{+}$and $\mathrm{MO}^{+}$species were observed at levels characteristic of the plasma. 
In 1996 Cleland and Meeks [14] used a similar technique to determine the most probable formation mechanism for $\mathrm{Ar}_{2}{ }^{+}$. They found a $\mathrm{T}_{\text {gas }}$ at which experimental ion ratios equaled calculated ion ratios. Of the mechanisms investigated, they found that assuming $\mathrm{Ar}_{2}{ }^{+}$is made by collisions of neutral Ar with $\mathrm{Ar}^{+}$gave the most realistic $\mathrm{T}_{\text {gas }}$ of $2400 \mathrm{~K}$ [14].

In 2001, Houk and Praphairaskit used a related idea and reported the $\mathrm{T}_{\text {gas }}$ at which experimental ion ratios equaled ion ratios calculated at various $T_{\text {gas }}$ values [3]. They postulated that the $\mathrm{T}_{\text {gas }}$ value measured in this way would indicate the region of the instrument where final dissociation equilibrium was established. They argued that, at the very least, comparison between polyatomic ions would be instructive. In 2006 Ferguson et al. expanded this work to investigate the effect of operating conditions on $\mathrm{T}_{\text {gas }}$, to incorporate high resolution and accurate mass measurements for definitive identification of the ions, and to apply the method to some additional polyatomic ions not considered previously, e.g. $\mathrm{C}_{\mathrm{x}} \mathrm{H}_{\mathrm{y}} \mathrm{O}^{+}[15-17]$.

In 2002, Evans et al. used this same technique along with two variations of Boltzmann plots [18]. Evans' group used only a few of the polyatomic ions listed in Nonose et al., and Houk and Praphairaksit. Results for $\mathrm{ArO}^{+}$were similar to those found by others; additional $\mathrm{ArO}^{+}$was made after the plasma. $\mathrm{LaO}^{+}$gave a lower temperature than that found by Houk and Praphairaksit, although Evans et al. do not discuss their mass bias correction technique. The normal mass bias favors ions at higher masses, which would result in lower $\mathrm{T}_{\mathrm{gas}}$ values if these corrections were omitted.

The present work extends the method of Houk and Praphairaksit and critically assesses various refinements to it. In particular, the influence of high-temperature 
corrections to the partition function, ionization reactions, excited electronic states, and errors in the various measured quantities are examined.

\section{Theory}

2.1 Origin of Polyatomic Ion from Measured $T_{\text {gas }}$ Values [3,19-22]

Previous methods use a dissociation reaction for the polyatomic ion. Assume a generic polyatomic ion, $\mathrm{AB}^{+}$, with a dissociation energy, $\mathrm{D}_{\mathrm{o}}$, and where $\mathrm{A}$ may be one or more atoms and $\mathrm{B}$ is either $\mathrm{Ar}, \mathrm{O}$ or $\mathrm{H}$,

$$
\mathrm{AB}^{+} \rightleftharpoons \mathrm{A}^{+}+\mathrm{B} \quad \Delta \mathrm{E}=\mathrm{D}_{0}
$$

The dissociation constant, $\mathrm{K}_{\mathrm{d}}$, for this reaction can be found in two ways, by measuring number densities, $n$, and by calculation with partition functions, $\mathrm{Z}$, at a given temperature.

$$
\frac{n_{A^{+}} n_{B}}{n_{A B^{+}}}=\mathrm{K}_{\mathrm{d}}=\frac{Z_{A^{+}} Z_{B}}{Z_{A B^{+}}} e^{-\mathrm{D}_{0} / \mathrm{k}_{\mathrm{B}} \mathrm{T}_{\text {gas }}}
$$

where $\mathrm{k}_{\mathrm{B}}=$ Boltzmann constant. The $\mathrm{T}_{\text {gas }}$ value that gives best agreement between the two sides is considered to be indicative of the region where the polyatomic ion is made. Most measurements are done with the sampler at the sampling position that gives highest atomic ion signal, which is the position most commonly used in analysis. This spot is consistently found to be at $\mathrm{T}_{\text {gas }}$ of $\sim 5000$ to $6000 \mathrm{~K}[3,15-17]$. 
The three common diagnoses for the origin of a given polyatomic ion are as follows. 1) If the measured $T_{\text {gas }}$ values fall within this range $(5000$ to $6000 \mathrm{~K})$, the ion $\mathrm{AB}^{+}$is observed at an abundance consistent with it being present in plasma.

2) If $\mathrm{T}_{\text {gas }}<5000 \mathrm{~K}$ there is an excess of $\mathrm{AB}^{+}$relative to that expected from the plasma itself. Some process(s) make additional $\mathrm{AB}^{+}$as the gas is extracted from the plasma. Some possible causes of $\mathrm{T}_{\text {gas }}$ values lower than those in the ICP could be reactions that make or remove ions in the following locations:

a) reactions early in the supersonic expansion that essentially stop when collisions become infrequent after the $\mathrm{M}=0.7$ position, where $\mathrm{T}_{\text {gas }}$ is $\sim 3000 \mathrm{~K}$ [13], b) cooling inside the sampler nozzle induced by thermal contact between the extracted gas and the metal sampler cone [23]

c) the boundary layer around the inside edge of the sampler hole, if the skimmer hole is large enough to sample some of this cooled gas;[24] or

d) shock waves at or inside the skimmer tip that reheat the extracted gas and generate more collisions [25-30]. This latter effect also induces a bimodal velocity distribution upon the extracted species.[30,31]

3) If $\mathrm{T}_{\text {gas }}>6000 \mathrm{~K}$, less $\mathrm{AB}^{+}$is being measured compared to that expected from the plasma. Some process causes loss of $\mathrm{AB}^{+}$relative to $\mathrm{A}^{+}$.

\subsection{Mass Bias Corrections and Number Densities of Neutral Species}

When using the measured ion ratio a correction should be done to account for mass bias in the instrument. We use an instrument response curve based on Ingle et al. [3,15,32]. To calculate neutral B number density, entrained air is neglected; we use a sampling position 
close to the end of the outer tube of the torch for this reason. Thus, the major constituents of the plasma are argon, oxygen and hydrogen from the plasma and aqueous sample solution. These three elements have low ionization efficiencies in the plasma, $0.04 \%, 0.1 \%$ and $0.1 \%$ respectively, so they are present almost totally as neutrals [1]. The total number density in the plasma is calculated from the Ideal Gas Law.

$$
\mathrm{n}_{\text {tot }}=\mathrm{n}_{\mathrm{Ar}}+\mathrm{n}_{\mathrm{O}}+\mathrm{n}_{\mathrm{H}}=\mathrm{P} / \mathrm{RT}_{\text {gas }}
$$

Neutral oxygen and hydrogen atoms are assumed to come from the nearly complete dissociation of water. If the amount of water load into the ICP torch per unit time is measured and the sample carrier gas flow rate is known, $n_{\mathrm{o}}$ can be calculated by the following:

$$
n_{O}=\frac{\mathrm{N}_{\mathrm{A}} \mathrm{LT}_{\text {room }}}{\mathrm{FM}_{\mathrm{H}_{2} \mathrm{O}} \mathrm{T}_{\mathrm{gas}} 1000}
$$

where $\mathrm{N}_{\mathrm{A}}$ is Avogadro's number, $\mathrm{L}$ is the measured solvent load $\left(\mathrm{g} \mathrm{min}^{-1}\right), \mathrm{T}_{\text {room }}$ is the room temperature $(298 \mathrm{~K}), \mathrm{F}$ is the sample gas flow rate $\left(1 \mathrm{~min}^{-1}\right)$ and $\mathrm{M}$ is the molar mass of water $\left(\mathrm{g} \mathrm{mol}^{-1}\right)$.

Once the number density of oxygen is known the density of the other neutrals can be found,

$$
\mathrm{n}_{\mathrm{H}}=2 \mathrm{n}_{\mathrm{O}}
$$




$$
\mathrm{n}_{\mathrm{Ar}}=\mathrm{P} / \mathrm{RT}_{\text {gas }}-3 \mathrm{n}_{\mathrm{O}}
$$

\subsection{High Temperature Corrections to the Partition Functions}

Partition functions come from the denominator of the Boltzmann equation,

$$
\mathrm{z}=\sum_{\mathrm{i}=0}^{\infty} \mathrm{g}_{\mathrm{i}} \mathrm{e}^{-\varepsilon_{\mathrm{i}} / \mathrm{k}_{\mathrm{B}} \mathrm{T}_{\mathrm{gas}}}
$$

where $\varepsilon_{\mathrm{i}}$ and $\mathrm{g}_{\mathrm{i}}$ are the energy and degeneracy of the ith level. Energy level equations are derived using the time-independent Schroedinger equation and simple models. For a molecule, the energy levels are treated as the sum of the translational, electronic, vibrational and rotational energies. The total partition function $\mathrm{Z}$ then becomes a product.

$$
Z=z_{\text {elec }} z_{\text {trans }} z_{\text {vib }} z_{\text {rot }}
$$

Translational energy levels are addressed simply and accurately with the particle-in-a-box model and the resulting partition function works for both atoms and molecules.

Electronic partition functions for atoms and atomic ions have been conveniently computed in the form of polynomials for temperatures ranging from $1500 \mathrm{~K}$ to $\sim 12,000 \mathrm{~K}$ [33-35]. For molecules the first excited electronic state is usually high enough in energy that it does not contribute significantly to the numerical values of the electronic partition function. Statistical thermodynamics texts recommend addition of electronic levels other than ground state only if highly accurate calculations are being done, there is an unusually low-lying excited state, or high temperatures are being used [19,22]. Because $\varepsilon_{0}$ can be set to zero, the 
molecular electronic partition function often simplifies to the degeneracy of the ground state which is approximated by the multiplicity for sigma terms and two times the multiplicity for all other terms $[19,22]$.

Vibrational and rotational energy levels come from the harmonic oscillator and rigid rotor models. For diatomic ions the Morse oscillator and the vibrating rotor provide more accurate predictions of vibrational-rotational spectra, but agreement between the various models is good for all but the most precise calculations, as long as the majority of molecules remain in low vibrational levels. As temperature increases and higher levels are populated, results from the two sets of models will gradually diverge leading to larger errors in the overall partition function. Since the ICP is at high temperatures, such corrections should be investigated.

For diatomic ions at high temperatures, three corrections can be applied to the energy levels of the harmonic oscillator and rigid rotor $[19,36]$.

1. Addition of centrifugal stretching constants $\left(D_{e}, \ldots\right)$ to account for the increased rotational energy increasing the centripetal force and stretching the internuclear bond.

$$
E_{r o t}=\mathrm{B} h c J(J+1)-\mathrm{D}_{\mathrm{e}} h c J^{2}(J+1)^{2}+\ldots
$$

$E_{r o t}$ is rotational energy, B is the rotational constant, $h$ is Planck's constant, $c$ is the speed of light and $J$ is the rotational quantum number.

2. Addition of anharmonicity constants $\left(x_{e}, y_{e}, \ldots\right)$ to help correct for deviation from Morse oscillator behavior:

$$
E_{v i b}=h c \omega\left(v+\frac{1}{2}\right)-x_{e} h c \omega\left(v+\frac{1}{2}\right)^{2}+y_{e} h c \omega\left(v+\frac{1}{2}\right)^{3}+\ldots
$$

where $v$ is the vibrational quantum number and $\omega$ is the vibrational constant. 
3. Addition of coupling constants $(\alpha, \beta, \ldots)$ because rotation and vibration are not completely independent. In the Morse oscillator, changing vibrational levels changes the mean internuclear distance, thus changing the moment of inertia and the rotational constant. $\mathrm{B}$ and $\mathrm{D}_{\mathrm{e}}$ from equation 9 then become $\mathrm{B}_{\mathrm{v}}$ and $\mathrm{D}_{\mathrm{v}}$ where,

$$
\begin{aligned}
& \mathrm{B}_{\mathrm{v}}=\mathrm{B}-\alpha\left(v+\frac{1}{2}\right)+\ldots \\
& \mathrm{D}_{\mathrm{v}}=\mathrm{D}_{\mathrm{e}}+\beta\left(v+\frac{1}{2}\right)+\ldots
\end{aligned}
$$

Unless very precise calculations are needed, the expansions are truncated after the first correction terms, $\mathrm{D}_{\mathrm{e}}, x_{\mathrm{e}}$ and $\alpha$.

\subsection{Correction for Ionization Reactions}

The previous work approached the polyatomic ion dissociation reaction as isolated from any additional equilibria considerations. In particular, the extent of ionization for neutral polyatomic and atomic ions was not incorporated. It was assumed that the relative number densities of $\mathrm{AB}^{+}$and $\mathrm{A}^{+}$would still reflect the position of an "equilibrated" dissociation reaction, even if the densities and measured signals for $\mathrm{A}^{+}$and $\mathrm{AB}^{+}$were also affected by ionization reactions. Also, for some species overall number densities are much larger than losses to ionization, formation and dissociation reactions.

One way to test the impact of considering multiple reactions is to expand reaction 1 into three reactions using a thermodynamic cycle. 


$$
\begin{array}{ll}
\mathrm{AB} \rightleftharpoons \mathrm{A}+\mathrm{B} & \mathrm{K}_{\mathrm{d}}(\mathrm{AB})=f\left(\mathrm{~T}_{\mathrm{gas}}\right) \\
\mathrm{A} \rightleftharpoons \mathrm{A}^{+}+\mathrm{e}^{-} & \mathrm{K}_{\text {ion }}(\mathrm{A})=f\left(\mathrm{~T}_{\text {ion }}\right) \\
\mathrm{AB}^{+}+\mathrm{e}^{-} \rightleftharpoons \mathrm{AB} & 1 / \mathrm{K}_{\text {ion }}(\mathrm{AB})=f\left(\mathrm{~T}_{\text {ion }}\right)
\end{array}
$$

where $\mathrm{T}_{\text {ion }}=$ ionization temperature. The dissociation constant for reaction 1 can then be calculated by multiplying the constants of reactions 13-15. Note that Eqns. 14 and 15 use ionization constants $\mathrm{K}_{\text {ion }}$ that are functions of $\mathrm{T}_{\mathrm{ion}}$, which does not equal $\mathrm{T}_{\text {gas }}$ in the ICP $[1,37]$. The $\mathrm{K}_{\text {ion }}$ values can be calculated from the Saha equation, which is derived from equation 2 where neutral B is just replaced with an electron.

\section{Experimental Section}

Adjustments were done to the original calculations from 24 experimental runs outlined in another paper [38]. These trials spanned different cone materials (Ni, $\mathrm{Pt}, \mathrm{Al}$ and mixes), plasma conditions (hot vs. cold) and X vs $\mathrm{H}$ skimmer cone geometries. Data analysis and calculations were done in Microsoft Excel 2003 or 2007.

Partition functions, neutral B number densities, and dissociation constants were calculated iteratively in $10 \mathrm{~K}$ increments. Ionization constants were calculated using the Saha equation [1] and a $\mathrm{T}_{\text {ion }}$ value of $7000 \mathrm{~K}$ for hot plasma conditions. This value is a compromise between older values of $7500 \mathrm{~K}$ [1] and $6529 \mathrm{~K}$ from a newer study by Bayon et al.[37] For the ionization efficiencies shown in Table $3, \mathrm{n}_{\mathrm{e}}=1 \times 10^{15} \mathrm{~cm}^{-3}$ was used. This 
value is derived from previous optical and Langmuir probe measurements on the gas just outside the sampler for plasmas operated at similar power and gas flow rates as those used in the present work. $[28,39]$ For cold plasma conditions $T_{\text {ion }}=4750 \mathrm{~K}$ and a much lower $n_{e}$ value $\left(4 \times 10^{10} \mathrm{~cm}^{-3}\right)$ were used. [10] Of course, all these $T_{\text {ion }}$ values were measured using atomic ions only; as a starting point, they are assumed to be valid for polyatomic ions as well.

Instrument response curves for hot plasma conditions take blank subtracted intensities divided by isotope abundance, ionization efficiency in the plasma,[1] and concentration in solution and plot the response versus $\mathrm{m} / \mathrm{z}$. Under cold plasma conditions ionization efficiencies were recalculated at the $\mathrm{T}_{\mathrm{ion}}$ and $\mathrm{n}_{\mathrm{e}}$ values given In the preceding paragraph. However, the resulting points scattered badly on the response curves; a polynomial line could only be fit to an $\mathrm{R}^{2}$ of 0.2748 . When ionization efficiency was ignored or hot plasma efficiencies were used a polynomial could be fit to the mass bias plot with a much better value of $\mathrm{R}^{2}=0.9671$. Therefore, instrument response curves for cold plasma runs were calculated in the same manner as for hot plasma runs.

High temperature corrections for diatomic molecules were done by using the following equation [19] for the rotational and vibrational partition functions. Pitzer's appendix was also useful in understanding this derivation [36].

$$
z_{r v}^{\prime}=\frac{1}{\sigma y}\left(\frac{1}{1-e^{-t}}\right)\left(1+\frac{y}{3}+\frac{2 \gamma}{y}+\frac{\delta}{e^{t}-1}+\frac{2 x_{e} t}{\left(e^{t}-1\right)^{2}\left(1-2 x_{e}\right)}\right)
$$


Here $\sigma$ is the symmetry number of the diatomic molecule. Heteronuclear diatomic ions have $\sigma=1$ and homonuclear diatomic ions have $\sigma=2$. Symbols $y, t, \gamma$ and $\delta$ are substitution constants as follows,

$$
\begin{aligned}
& y=\frac{h c}{k T}\left(\mathrm{~B}-\frac{1}{2} \alpha\right) \\
& t=\frac{h c}{k T}\left(\omega-2 \omega x_{e}\right) \\
& \gamma=\frac{\mathrm{D}_{\mathrm{e}}}{\left(\mathrm{B}-\frac{1}{2} \alpha\right)} \\
& \delta=\frac{\alpha}{\left(\mathrm{B}-\frac{1}{2} \alpha\right)}
\end{aligned}
$$

As can be seen, only the first order corrections for centripetal stretching, $D_{e}$, anharmonicity, $\chi_{\mathrm{e}}$, and vibrational stretching, $\alpha$, are used. These constants are often reported with basic spectroscopic data [40] but can also be calculated as done in the present work.

$$
\begin{aligned}
& \chi_{\mathrm{e}}=\frac{\mathrm{hc} \omega}{4 \mathrm{D}} \\
& \mathrm{D}_{\mathrm{e}}=\frac{4 \mathrm{~B}^{2}}{\omega^{2}}
\end{aligned}
$$




$$
\alpha=\frac{6 \mathrm{~B}^{2}}{\omega}\left[\left(\frac{\chi_{\mathrm{e}} \omega}{\mathrm{B}}\right)^{1 / 2}-1\right]
$$

$D$ is well depth or the dissociation energy plus the zero point energy.

Equation 16 is the result of truncating after the first or second terms of the EulerMaclaurin expansions of the energy level summations. Preliminary work with MATLAB (MathWorks, Natick, Massachusetts) showed no effect when additional terms were added.

\section{Results and Discussion}

\subsection{Electronic Partition Functions of Diatomic Molecules}

Table $\square$ lists ground and excited electronic states for each of the diatomic background ions studied in this paper (i.e., excluding $\mathrm{CeO}^{+}$and $\mathrm{ScO}^{+}$), the energy difference between the ground and first excited electronic levels [40-44] and any error in the electronic partition function that comes from not adding the first excited electronic state. The ground electronic state assumption at $5000 \mathrm{~K}$ gives a less than $5 \%$ difference in the partition function value for every diatomic background ion except $\mathrm{ArO}^{+}$. Determination of reasonable, consistent $\mathrm{T}_{\text {gas }}$ values using $\mathrm{ArO}^{+}$proved to be problematic, as discussed in an upcoming paper [45].

In equations 13 and 15 the partition function for the neutral diatomic molecule is necessary. Table 2 lists the first two excited states of some neutral diatomic molecules and their contributions to the electronic partition function. Two species have a important low lying levels. $\mathrm{O}_{2}$ has ${ }^{3} \Sigma$ ground state and ${ }^{1} \Delta$ excited electronic state at $7,918 \mathrm{~cm}^{-1}$. For NO 
ground state ${ }^{2} \Pi_{1 / 2}$ has the spin-orbit state ${ }^{2} \Pi_{3 / 2}$ at only $120 \mathrm{~cm}^{-1}$. In fact, these two molecules are often given as cases when excited state populations need to be included, even at room temperature [19,21]. Therefore calculations with these two neutral diatomics always included the first excited electronic states.

\subsection{Effect of High Temperature Rotational-Vibrational Corrections to Partition Functions}

The high temperatures of 5000 to $6000 \mathrm{~K}$ at the sampling point in the ICP are well above the warning line of 3000 to $4000 \mathrm{~K}$ commonly given in statistical thermodynamics texts for requirement of high-temperature corrections to partition functions [19]. Yet when equation 16 was used with experimentally-measured signal ratios, there were only minimal changes in the resulting $T_{\text {gas }}$ values compared to simpler calculation models $[3,15]$. Measured $\mathrm{T}_{\text {gas }}$ values using $\mathrm{Ar}_{2}{ }^{+}, \mathrm{ArH}^{+}, \mathrm{O}_{2}{ }^{+}$, or $\mathrm{ScO}^{+}$never changed more than $10 \mathrm{~K}$ for all 23 trials. $\mathrm{ArN}^{+}$and $\mathrm{OH}^{+}$showed a $10 \mathrm{~K}$ increase only once. $\mathrm{CeO}^{+}$and $\mathrm{NO}^{+}$gave the largest $\mathrm{T}_{\text {gas }}$ values in this work and therefore would be expected to need the temperature correction most. The largest changes in $\mathrm{T}_{\text {gas }}$ were seen using these latter two ions with increases by up to $40 \mathrm{~K}$, although this would still not change the diagnoses of their origins [38].

Because the main goal of this work is to estimate ion origin and such minor changes in $\mathrm{T}_{\text {gas }}$ do not affect these diagnoses, high temperature corrections were not used further. Including them does not change the total partition function enough to affect the basic diagnosis of the origin of the polyatomic ion.

\subsection{Inclusion of Ionization Energy for Neutral Diatomic Molecules}


$\mathrm{T}_{\text {gas }}$ values from $\mathrm{O}_{2}{ }^{+}, \mathrm{NO}^{+}, \mathrm{OH}^{+}, \mathrm{CeO}^{+}$and $\mathrm{ScO}^{+}$were examined for the effect of including ionization reactions. Table 3 lists the ionization energies [46-48] and the percent ionization of each species at $T_{\text {ion }}=7000 \mathrm{~K}$ and an electron density of $1 \times 10^{15} \mathrm{~cm}^{-3}[39]$. The results are given in Figure 1 for a hot plasma and in Figure 2 for a cool plasma. Note that $n_{e}$ was only used to calculate the degree of ionization values in Table 3 . When $\mathrm{T}_{\text {gas }}$ is evaluated from Equations 14 and 15, the $\mathrm{n}_{\mathrm{e}}$ values cancel.

In the hot plasma trials (Figure 1) no species changed its essential behavior when ionization was included. $\mathrm{CeO}^{+}$and $\mathrm{O}_{2}{ }^{+}$still gave $\mathrm{T}_{\text {gas }}$ values characteristic of the plasma at the sampling point. $\mathrm{NO}^{+}$still gave a high temperature indicating less $\mathrm{NO}^{+}$than expected. Thus, including ionization is not the main cause of the high $\mathrm{T}_{\text {gas }}$ values seen for $\mathrm{NO}^{+}$, on both this sector instrument and in previous studies with a home-made quadrupole device.[3] $\mathrm{OH}^{+}$ still gave a low temperature indicating more $\mathrm{OH}^{+}$than expected given plasma conditions alone.

Under hot plasma conditions, the corrected signal ratio $\mathrm{ScO}^{+} / \mathrm{Sc}^{+}$gives almost the same value of $\mathrm{T}_{\text {gas }}$ as $\mathrm{CeO}^{+} / \mathrm{Ce}^{+}$, both in this work (data not shown) and in previous experiments.[3] Therefore, $\mathrm{ScO}^{+} / \mathrm{Sc}^{+}$was used to give the estimated temperature range for the plasma at the sampling point for the cool plasma; $\mathrm{CeO}^{+} / \mathrm{Ce}^{+}$was not used because there was very little $\mathrm{Ce}^{+}$signal from the cool plasma. Inclusion of ionization energies led to similar changes as described above (Figure 2). Average $\mathrm{T}_{\text {gas }}$ values changed slightly, but overall diagnoses did not for most ions. The two possible exceptions are $\mathrm{NO}^{+}$and $\mathrm{O}_{2}{ }^{+}$. The average $\mathrm{T}_{\text {gas }}$ for $\mathrm{NO}^{+}$dropped by $240 \mathrm{~K}$. The black box in Figure 2 shows an approximate $1000 \mathrm{~K}$ difference with the center on the $\mathrm{T}_{\text {gas }}$ value from $\mathrm{ScO}^{+}$. $\mathrm{NO}^{+}$goes from just above this box to just below the top line of this box. This could change the diagnosis for $\mathrm{NO}^{+}$from 
loss of polyatomic ion after extraction to $\mathrm{NO}^{+}$being present at levels expected in the plasma. Alternatively, it is likely that the expected temperature spread for the plasma would also be smaller under cool conditions, the range of $\mathrm{T}_{\text {gas }}$ values seen for ions from the plasma shrinks, so the abundance of $\mathrm{NO}^{+}$still reflects some loss in the extraction process.

By the same consideration $\mathrm{O}_{2}{ }^{+}$could change its diagnosis from plasma levels to excess polyatomic ion. However, the argument from $\mathrm{NO}^{+}$applied here would leave $\mathrm{O}_{2}{ }^{+}$ firmly in the latter category for both calculation methods.

To summarize, inclusion of ionization reactions in the calculated $\mathrm{K}_{\mathrm{d}}$ does change the $\mathrm{T}_{\text {gas }}$ value from some polyatomic ions but not to a degree outside the day to day variations and not so much that the diagnosis of the probable origin of the ion changes.

\subsection{Error Analysis}

Error analysis in these calculations is not a matter of simple error propagation. Iteration is used precisely because there is no straightforward equation to find $\mathrm{T}_{\text {gas }}$ from the measured signal ratio and $\mathrm{K}_{\mathrm{d}}$. Therefore, to test the effect of change in one variable, the $\mathrm{T}_{\text {gas }}$ estimate procedure was repeated with the "correct" value of one given parameter increased by $10 \%$. In this way the effect of $10 \%$ errors in each of solvent load, sample gas flow rate, vibrational constant, rotational constant, dissociation energy, and the measured ion ratio were investigated for one day's data.

Results from this error analysis are presented in Table 4. On the day under consideration, nickel cones, an H-geometry skimmer, and 'hot' plasma conditions were used. As expected from the form of Equation (2), the dissociation energy has the largest impact. A $10 \%$ change in $\mathrm{D}_{\mathrm{o}}$ leads to about $10 \%$ changes in temperature. Thus, reasonably 
accurate values for this quantity are necessary. Changing solvent load, sample gas flow rate, vibrational constants, rotational constants and even ion ratios by $10 \%$ usually produced errors in the $\mathrm{T}_{\text {gas }}$ only about a tenth of that (-140 to $40 \mathrm{~K}$ change, $0.7-3.1 \%$ of $\left.\mathrm{T}_{\text {gas }}\right)$. In general, such errors will not affect the diagnosis of ion origin.

\section{Acknowledgements}

This research was supported by the National Science Foundation (Award No. CHE0309381) through the Institute for Physical Research and Technology at ISU. The ICP-MS instrument was obtained with funds provided by the U. S. Department of Energy, Office of Nuclear Nonproliferation (NA-22) and the Office of Basic Energy Sciences.

\section{References}

[1] H. Niu, R.S. Houk, Fundamental aspects of ion extraction in inductively coupled plasmamass spectrometry, Spectrochim. Acta Part B 51 (1996) 779-815.

[2] D.J. Douglas, J.B. French, An improved interface for inductively coupled plasma mass spectrometry, Spectrochim. Acta B 41 (1986) 197-204.

[3] R.S. Houk, N. Praphairaksit, Dissociation of polyatomic ions in inductively coupled plasma, Spectrochim. Acta B 56 (2001) 1069-1096.

[4] N.S. Nonose, N. Matsuda, N. Fudagawa, M. Kubota, Some characteristics of polyatomic ion spectra in inductively coupled plasma-mass spectrometry, Spectrochim. Acta Part B 49 (1994) 955-974.

[5] A.E. Holliday, D. Beauchemin, Spatial profiling of analyte signal intensities in inductively coupled plasma mass spectrometry, Spectrochim. Acta B 59 (2004) 291-311. 
[6] J.T. Rowan, R.S. Houk, Attenuation of polyatomic ion interferences in inductively coupled plasma mass spectrometry by gas-phase collisions, Appl. Spectrosc. 43 (1989) 976980.

[7] S.D. Tanner, V.I. Baranov, D.R. Bandura, Reaction cells and collision cells for ICPMS:a tutorial review, Spectrochim. Acta B 57 (2002) 1361-1452.

[8] N. Bradshaw, E.F.H. Hall, N.E. Sanderson, Inductively coupled plasma as an ion source for high-resolution mass spectrometry, J. Anal. At. Spectrom. 8 (1989) 801-803. [9] S.J. Jiang, R.S. Houk, M.A. Stevens, Alleviation of overlap interferences for determination of potassium isotope ratios by inductively coupled plasma mass spectrometry, Anal. Chem. 60 (1988) 1217-1221.

[10] S.D. Tanner, Characterization of ionization and matrix suppression in inductively coupled 'cold' plasma mass spectrometry, J. Anal. At. Spectrom. 10 (1995) 905-921. [11] L.C. Alves, D.R. Wiederin, R.S. Houk, Reduction of polyatomic ion interferences in inductively coupled plasma mass spectrometry by cryogenic desolvation, Anal. Chem. 64 (1992) 1164-1169.

[12] M.G. Minnich, R.S. Houk, Comparison of cryogenic and membrane desolvation for attenuation of oxide, hydride and hydroxide ions and ions containing chlorine in inductively coupled plasma mass spectrometry, J. Anal. At. Spectrom. 13 (1998) 167-174.

[13] D.J. Douglas, J.B. French, Gas dynamics of the inductively coupled plasma mass spectrometer interface, J. Anal. At. Spectrom. 3 (1988) 743-747.

[14] T.J. Cleland, F.R. Meeks, Statistical mechanics of $\mathrm{Ar}_{2}{ }^{+}$in an inductively coupled plasma, Spectrochim. Acta B 51 (1996) 1487-1490.

[15] J.W. Ferguson, R.S. Houk, High resolution studies of the origins of polyatomic ions in inductively coupled plasma-mass spectrometry, Part 1. Identification methods and effects of neutral gas density assumptions, extraction voltage, and cone material, Spectrochim. Acta Part B 61 (2006) 905-915.

[16] J.W. Ferguson, T.J. Dudley, K.C. Sears, S.M. McIntyre, M.S. Gordon, R.S. Houk, Polyatomic ions in inductively coupled plasma-mass spectrometry Part II: Origins of $\mathrm{N}_{2} \mathrm{H}^{+}$ and $\mathrm{H}_{\mathrm{x}} \mathrm{CO}^{+}$ions using experimental measurements combined with calculated energies and structures, Spectrochim. Acta B 64 (2009) 690-696. 
[17] J.W. Ferguson, T.J. Dudley, M.S. Gordon, R.S. Houk, High resolution studies of the origins of polyatomic ions in ICP-MS Part III: $\mathrm{CrO}_{\mathrm{x}} \mathrm{H}_{\mathrm{y}}{ }^{+}$ions, manuscript in preparation (2011).

[18] E.H. Evans, L. Ebon, L. Rowley, Comparative study of the determination of equilibrium dissociation temperature in inductively coupled plasma-mass spectrometry, Spectrochim. Acta Part B 57 (2002) 741-754.

[19] R.E. Sonntag and G.J. Van Wylen, Fundamentals of Statistical Thermodynamics, John Wiley and Sons, Inc., New York, 1966.

[20] J.H. Knox, Molecular Thermodynamics: An Introduction to Statistical Mechanics for Chemists, Chapter 6, Rev. Ed. John Wiley and Sons, Chichester, 1978.

[21] K.J. Laidler, J.H. Meiser, Physical Chemistry, Chapter 15, Houghton Mifflin Co., Boston, 1999.

[22] N.M. Laurendeau, Statistical Thermodynamics: Fundamentals and Applications, Chapter 9, Cambridge University Press, Cambridge, 2005.

[23] R. L. Spencer, J. Krogel, J. Palmer, A. Payne, A. Sampson, W. Somers, C. N. Woods, Modeling the gas flow upstream and in the sampling nozzle of the inductively coupled plasma mass spectrometer via the Direct Simulation Monte Carlo algorithm, Spectrochim. Acta Part B 64 (2009) 215-221.

[24] M. A. Vaughan, G. Horlick, Effect of sampler and skimmer orifice size on analyte and analyte oxide signals in ICP-MS, Spectrochim. Acta Part B 45 (1990) 1289-1300.

[25] A. L. Gray, Visual observation of shock waves in an ICP-MS expansion stage, J. Anal. Atomic Spectrom. 4 (1989) 371-373.

[26] R. S. Houk, H. B. Lim, Atomic Emission Spectrometry with a Reduced Pressure Afterglow Extracted from an Inductively Coupled Plasma, Anal. Chem. 58 (1986) 32443248 .

[27] H.B. Lim, R.S. Houk, M.C. Edelson, K.P. Carney, Some fundamental characteristics of a reduced-pressure plasma extracted from an ICP, J. Anal. Atom. Spectrom. 4 (1989) 365370. 
[28] H. Niu, R. S. Houk, Langmuir probe measurements of the ion extraction process in inductively coupled plasma mass spectrometry-I. spatially resolved determination of electron density and electron temperature, Spectrochim. Acta Part B, 49 (1994) 1283-1303.

[29] T. N. Olney, W. Chen, D. J. Douglas, Gas dynamics of the ICP-MS interface: impact pressure probe measurements of gas flow profiles, J. Anal. Atomic Spectrom. 14 (1999) 917.

[30] W. N. Radicic, J. B. Olsen, R. V. Nielson, J. H. Macedone, P. B. Farnsworth, Characterization of the supersonic expansion in the vacuum interface of an inductively coupled plasma mass spectrometer by high-resolution diode laser spectroscopy, Spectrochim. Acta Part B 61 (2006) 686-695.

[31] V. Baranov, S. D. Tanner, Multi-thermal plasma distributions from ion kinetics energy measurements, Federation of Analytical Chemistry and Spectroscopy Societies (FACSS) Conference, Providence RI, October 1997, Paper No. 248.

[32] C.P. Ingle, B.L. Sharp, M.S.A. Horstwood, R.R. Parrish, D.J. Lewis, Instrument response functions, mass bias and matrix effects in isotope ratio measurements and semiquantitative analysis by single and multi-collector ICP-MS, J. Anal. At. Spect. 18 (2003) 219-229.

[33] L. De Galan, R. Smith, J.D. Winefordner, The electronic partition function of atoms and ions between $1500^{\circ} \mathrm{K}$ and $7000^{\circ} \mathrm{K}$, Spectrochim. Acta B 23 (1968) 521-525.

[34] S. Tamaki, T. Kuroda, The electronic partition functions of atoms and ions between 7000 and 12000 K, Spectrochim. Acta B 42 (1987) 1105-1111.

[35] B. Faggetter, G. Heisz, M.W. Blades, The electronic partition functions of lanthanide atoms and ions between 1500 and 8000 K, Spectrochim. Acta B 42 (1987) 1235-1236.

[36] K.S. Pitzer, Quantum Chemistry. Prentice-Hall, Inc. New York, 1953.

[37] M.M. Bayón, J.I.G. Alonso, A.S. Medel, Enhanced semiquantitative multi-analysis of trace elements in environmental samples using inductively coupled plasma mass spectrometry, J. Anal. At. Spect., 13 (1998) 277-282.

[38] S. M. McIntyre, J.W. Ferguson, R.S. Houk, Polyatomic ions in inductively coupled plasma-mass spectrometry: Effects of cone material, skimmer cone geometry and cold 
plasma conditions on calculated versus measured ion ratios, manuscript in preparation (2010).

[39] R.S. Houk, Y. Zhai, Comparison of mass spectrometric and optical measurements of temperature and electron density in the inductively coupled plasma during mass spectrometric sampling, Spectrochim. Acta B 56 (2001) 1055-1067.

[40] K.P. Huber, G. Herzberg, Molecular Spectra and Molecular Structure IV. Constants of Diatomic Molecules, Van Nostrand Reinhold, New York, 1979.

[41] W.R. Wadt, The electronic states of $\mathrm{Ar}_{2}^{+}, \mathrm{Kr}_{2}^{+}, \mathrm{Xe}_{2}^{+}$. I. Potential curves with and without spin-orbit coupling, J. Chem. Phys., 68 (1978) 402-414.

[42] L. Broström, M. Larsson, S. Mannervik, D. Donnek, The visible photoabsorption spectrum and potential curves of $\mathrm{ArN}^{+}$, J. Chem. Phys., 94 (1991) 2734-2740.

[43] A.V. Stolyarov, M.S. Child, Theoretical study of $\mathrm{ArH}^{+}$electronic states, Phys. Chem. Chem. Phys., 7 (2005) 2259-2265.

[44] H.Frenking, W. Koch, D. Cremer, J. Gauss, J.F.Liebman, Neon and Argon Bonding in First-Row Cations $\mathrm{NeX}^{+}$and $\mathrm{ArX}^{+}$(X = Li-Ne), J. Phys. Chem., 93 (1989) 3410-3418.

[45] S. M. McIntyre, J.W. Ferguson, R.S. Houk, Determination of dissociation temperature for $\mathrm{ArO}^{+}$in inductively coupled plasma-mass spectrometry, manuscript in preparation (2011).

[46] Periodic Table of the Elements, VWR Scientific Products Sargent Welch (1996) Buffalo Grove, IL.

[47] R.J. Ackermann, E.G. Rauh, R.J. Thorn, The thermodynamics of ionization of gaseous oxides; the first ionization potentials of the lanthanide metals and monoxides, J. Chem. Phys., 65 (1976) 1027-1031.

[48] D.E. Clemmer, J.L. Elkind, N. Aristov, P.B. Armentrout, Reaction of $\mathrm{Sc}^{+}, \mathrm{Ti}^{+}$, and $\mathrm{V}^{+}$ with CO. $\mathrm{MC}^{+}$and $\mathrm{MO}^{+}$bond energies, J. Chem. Phys. 95 (1991) 3387-3393. 
Table 1. Effect of including first excited state on the electronic partition function of certain charged diatomic molecules.

\begin{tabular}{|c|c|c|c|c|c|c|c|c|c|}
\hline \multirow[b]{3}{*}{ Ion } & \multicolumn{2}{|c|}{ Ground State } & \multicolumn{4}{|c|}{ First Excited Electronic State } & \multirow[b]{3}{*}{$\mathbf{z}_{\text {elec }}$} & \multirow{2}{*}{\multicolumn{2}{|c|}{ Difference }} \\
\hline & Term & $\mathbf{g}_{0}$ & Term & & Energy & & & & \\
\hline & Symbol & $\left(\mathbf{z}_{\text {elec }}\right)$ & Symbol & $\mathbf{g}_{1}$ & $\left(\mathrm{~cm}^{-1}\right)$ & $\mathbf{g}_{1} \mathrm{e}^{(-\mathrm{E} 1 / \mathrm{kT})}$ & & in $z_{\text {elec }}$ & Source \\
\hline $\mathrm{O}_{2}^{+}$ & ${ }^{2} \Pi$ & 4 & ${ }^{4} \Pi$ & 8 & 32,964 & 0.00060723 & 4.00060723 & 0.0152 & 34 \\
\hline $\mathrm{NO}^{+}$ & ${ }^{1} \Sigma$ & 1 & ${ }^{3} \Sigma$ & 3 & 52,190 & 0.00000090 & 1.00000090 & 0.00009 & 34 \\
\hline $\mathrm{OH}^{+}$ & ${ }^{3} \Sigma$ & 3 & ${ }^{1} \Delta$ & 2 & 17,660 & 0.01241478 & 3.01241478 & 0.4 & 34 \\
\hline $\mathrm{Ar}_{2}{ }^{+}$ & ${ }^{2} \Sigma$ & 2 & ${ }^{2} \Pi$ & 4 & 12,986 & 0.09531766 & 2.09531766 & 4.8 & 35 \\
\hline $\mathrm{ArN}^{+}$ & ${ }^{3} \Sigma$ & 3 & ${ }^{3} \Pi$ & 6 & 14,047 & 0.10534204 & 3.10534204 & 3.5 & 36 \\
\hline $\mathrm{ArH}^{+}$ & ${ }^{1} \Sigma$ & 1 & ${ }^{3} \Pi$ & 6 & 49,163 & 0.00000430 & 1.00000430 & 0.0004 & 37 \\
\hline $\mathrm{ArO}^{+}$ & ${ }^{4} \Sigma$ & 4 & ${ }^{2} \Pi$ & 4 & 3,490 & 1.46515850 & 5.46515850 & 36.6 & 38 \\
\hline
\end{tabular}


Table 2. Effect of including first excited state on the electronic partition function of certain neutral species. Fundamental data are from ref. 34.

\begin{tabular}{|c|c|c|c|c|c|c|c|c|}
\hline \multirow[b]{3}{*}{ Species } & \multirow{2}{*}{\multicolumn{2}{|c|}{$\begin{array}{c}\text { Ground State } \\
\text { Term }\end{array}$}} & \multicolumn{4}{|c|}{ First Excited State } & \multirow[b]{3}{*}{$\mathbf{Z}_{\text {elec }}$} & \multirow[b]{3}{*}{ \%error } \\
\hline & & & Term & & Energy & & & \\
\hline & Symbol & $\mathrm{g}_{0}\left(\mathrm{z}_{\text {elec }}\right)$ & Symbol & $\mathrm{g}_{1}$ & $\left(\mathrm{~cm}^{-1}\right)$ & $\mathrm{g}_{1} \mathrm{e}^{(-\mathrm{E} 1 / \mathrm{kT})}$ & & \\
\hline $\mathrm{O}_{2}$ & ${ }^{3} \Sigma$ & 3 & ${ }^{1} \Delta$ & 2 & 7,918 & 0.20485707 & 3.20485707 & 6.8 \\
\hline NO & ${ }^{2} \Pi_{1 / 2}$ & 4 & ${ }^{2} \Pi_{3 / 2}$ & 4 & 120 & 3.86442842 & 7.86442842 & 96.6 \\
\hline $\mathrm{OH}$ & ${ }^{2} \Pi$ & 4 & ${ }^{2} \Sigma$ & 2 & 32,684 & 0.00016454 & 4.00016454 & 0.0 \\
\hline $\mathrm{ScO}$ & ${ }^{2} \Sigma$ & 2 & ${ }^{2} \Delta$ & 4 & 15,030 & 0.05292773 & 2.05292773 & 2.6 \\
\hline
\end{tabular}


Table 3. Ionization energies and ionization efficiencies for atoms and neutral polyatomic ions under hot plasma conditions.

\begin{tabular}{|c|c|c|c|}
\hline \multirow{2}{*}{ Species } & $(\mathbf{e V})$ & \% Ionization $^{\mathbf{a}}$ & Reference \\
\hline $\mathrm{O}_{2}$ & 12.071 & 0.5 & 34 \\
\hline $\mathrm{O}$ & 13.618 & 0.02 & 40 \\
\hline $\mathrm{OH}$ & 12.9 & 0.1 & 34 \\
\hline $\mathrm{NO}$ & 9.26436 & 5 & 34 \\
\hline $\mathrm{N}$ & 14.534 & 0.02 & 40 \\
\hline $\mathrm{CeO}$ & 4.9 & 100 & 40 \\
\hline $\mathrm{Ce}$ & 5.47 & 100 & 42 \\
\hline $\mathrm{ScO}$ & 6.6 & 100 & 40 \\
\hline $\mathrm{Sc}$ & 6.54 & 100 & \\
\hline
\end{tabular}

${ }^{\mathrm{a}}$ Calculated from Saha Eqn at $\mathrm{T}_{\mathrm{ion}}=7000 \mathrm{~K}, \mathrm{n}_{\mathrm{e}}=1 \times 10^{15} \mathrm{~cm}^{-3}$.

$\%$ Ionization $=\frac{\mathrm{n}_{\mathrm{A}^{+}}}{\mathrm{n}_{\mathrm{A}^{+}}+\mathrm{n}_{\mathrm{A}}} \times 100 \%$ 
Table 4. Error analysis of one run. Hot plasma mode, Ni cones, H-skimmer geometry.

Measured $\mathrm{T}_{\text {gas }}$ Values (K), Difference (K) and \% Difference

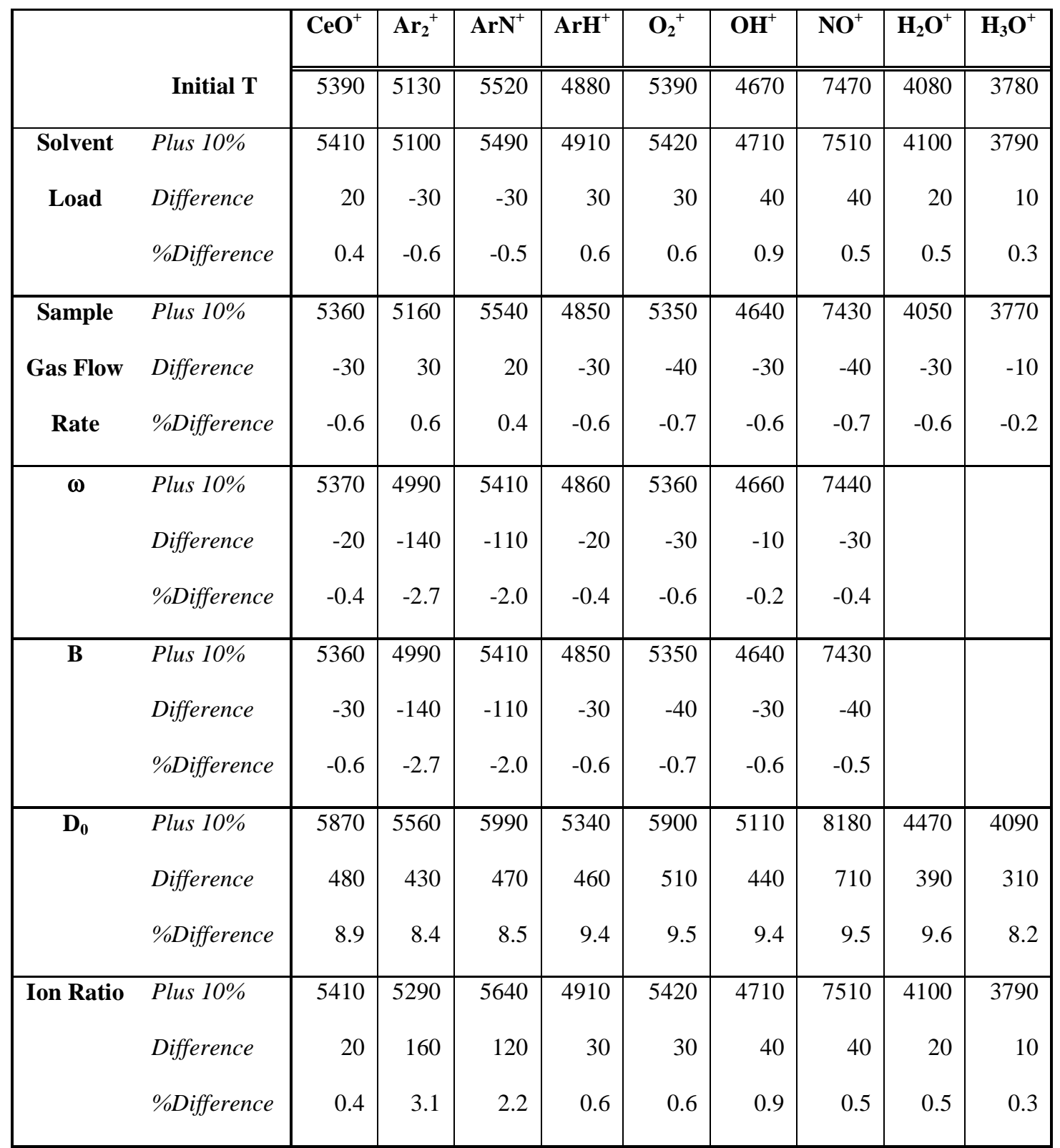




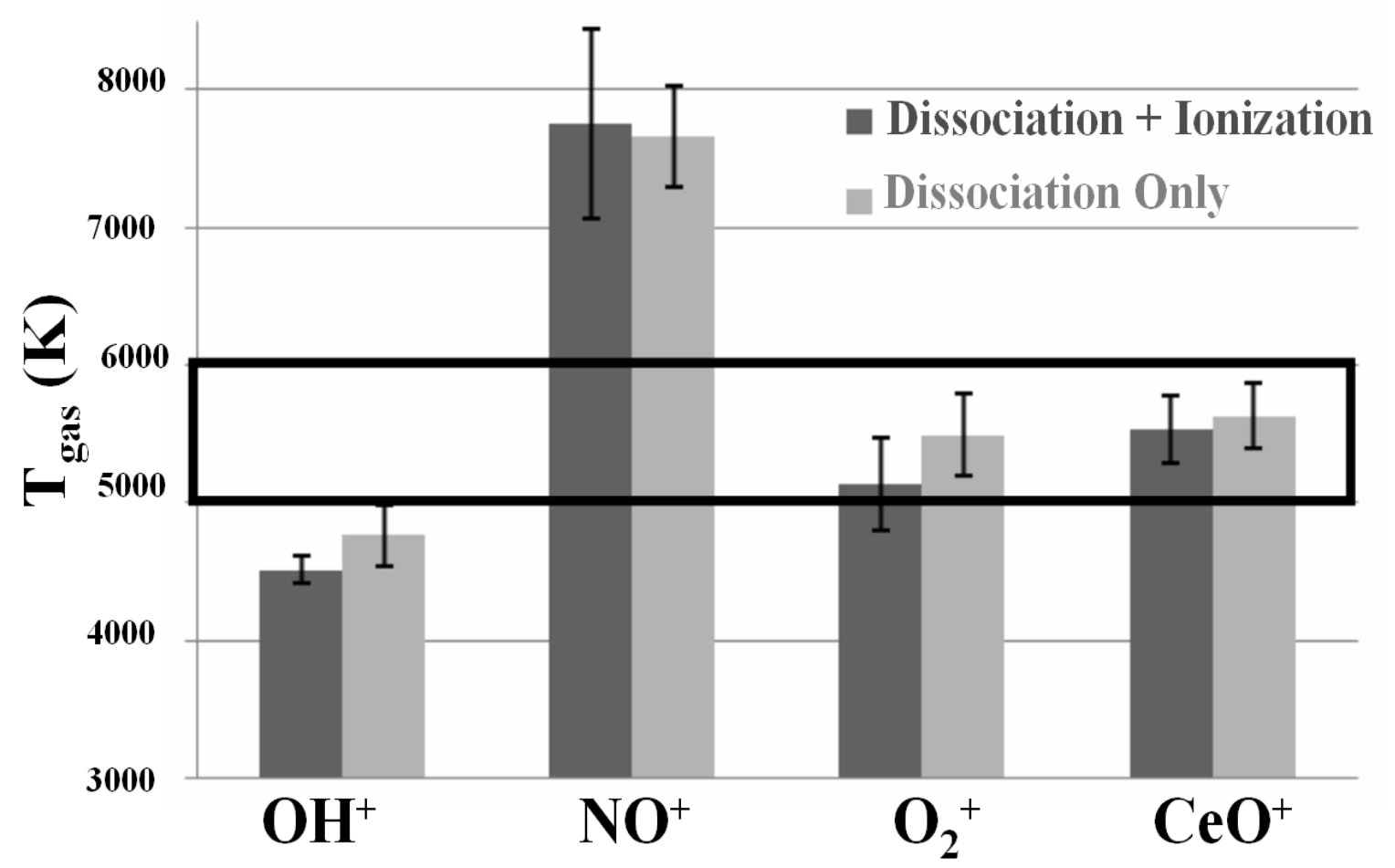

Figure 1. Gas kinetic temperature calculated setting Equations 2 and 3 equal to each other (new, Dissociation Only) and calculated including ionization energy of the polyatomic molecule and charged dissociation product (Equations 8-10, New, Dissociation + Ionization) in a hot plasma. Box represents the typical range of plasma temperatures at the sampling point $(5000$ to $6000 \mathrm{~K})$. $\mathrm{n}=20$ and error bars correspond to standard deviation. 


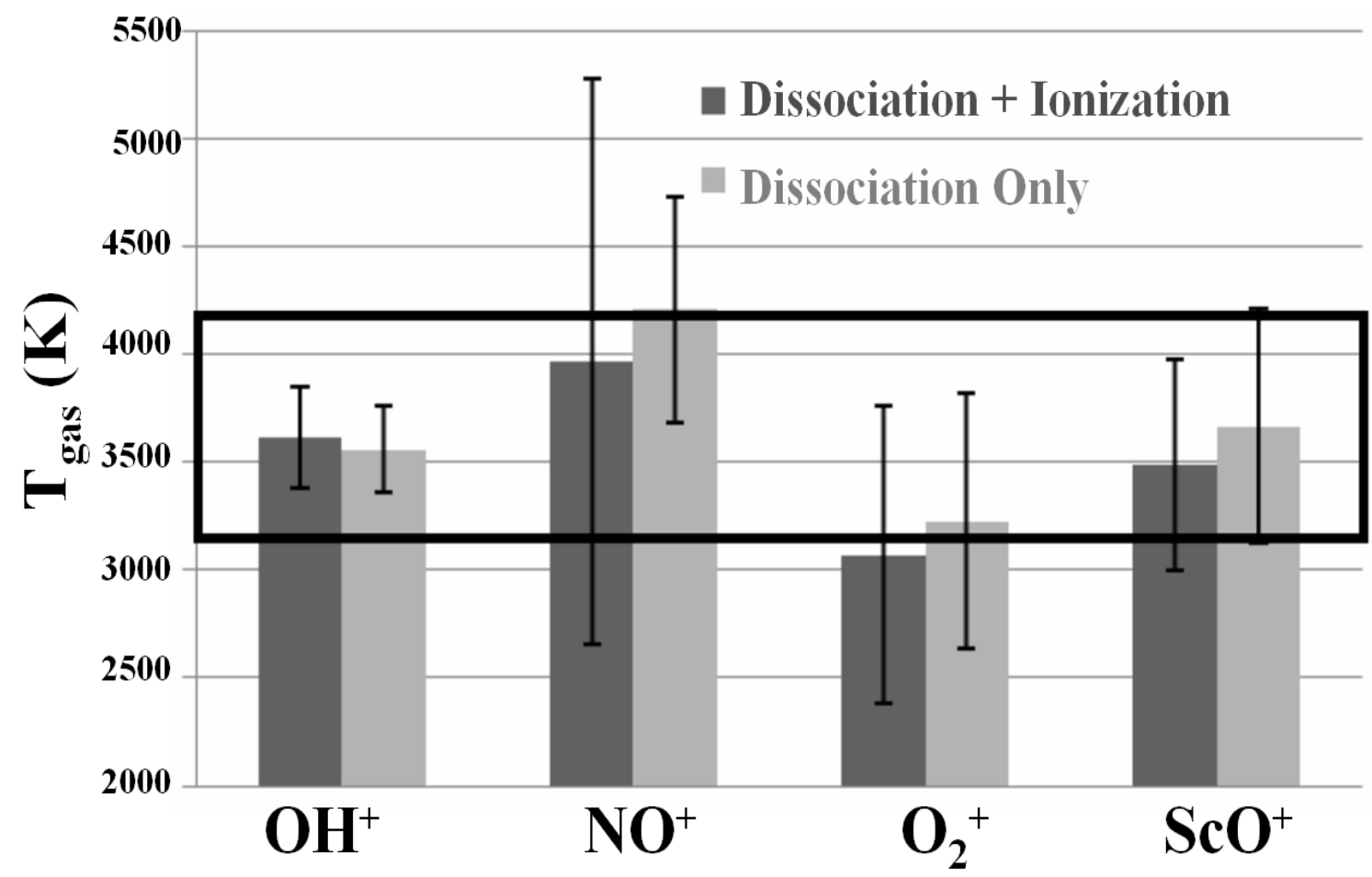

Figure 2. Gas kinetic temperature calculated setting Equations 2 and 3 equal to each other (Dissociation Only) and calculated including ionization energy of the polyatomic molecule and charged dissociation product (Equations 8-10, Dissociation + Ionization) in a 'cold' plasma. Assuming the metal oxide gives a characteristic temperature of the plasma, the box represents a $1000 \mathrm{~K}$ range for the plasma temperature. Sixteen spectra on each of three days were averaged. Error bars correspond to range over the three days 


\title{
Appendix B.
}

\section{Vanadium Induces Dopaminergic Neurotoxicity Via Protein Kinase C- Delta Dependent Oxidative Signaling Mechanisms: Relevance to Etiopathogenesis of Parkinson's Disease}

\author{
Afeseh Ngwa, Hilary ${ }^{1}$; Kanthasamy, Arthi ${ }^{1}$; Anantharam, Vellareddy ${ }^{1}$; \\ Song, Chunjuan ; Witte, Travis ${ }^{2}$; Houk R. S. ${ }^{2}$; Kanthasamy, Anumantha G. ${ }^{1}$ \\ ${ }^{1}$ Department of Biomedical Sciences, Iowa Center for Advanced Neurotoxicology, \\ Iowa State University, Ames, IA 50011, USA \\ ${ }^{2}$ Ames Laboratory, U. S. Department of Energy, Department of Chemistry, \\ Iowa State University, Ames, Iowa 50011 USA
}

A paper published in Toxicology and Applied Pharmacology, 2009, 240, 273-285. 


\begin{abstract}
$\underline{\text { Abstract }}$
Environmental exposure to neurotoxic metals through various sources including exposure to welding fumes has been linked to an increased incidence of Parkinson's disease (PD). Welding fumes contain many different metals including vanadium typically present as particulates containing vanadium pentoxide $\left(\mathrm{V}_{2} \mathrm{O}_{5}\right)$. However, possible neurotoxic effects of this metal oxide on dopaminergic neuronal cells are not well studied. In the present study, we characterized vanadium-induced oxidative stress-dependent cellular events in cell culture models of PD. $\mathrm{V}_{2} \mathrm{O}_{5}$ was neurotoxic to dopaminergic neuronal cells including primary nigral dopaminergic neurons and the $\mathrm{EC}_{50}$ was determined to be $37 \mu \mathrm{M}$ in $\mathrm{N} 27$ dopaminergic neuronal cell model. The neurotoxic effect was accompanied by a time-dependent uptake of vanadium and upregulation of metal transporter proteins Tf and DMT1 in N27 cells. Additionally, vanadium resulted in a threefold increase in reactive oxygen species generation, followed by release of mitochondrial cytochrome c into cytoplasm and subsequent activation of caspase-9 (>fourfold) and caspase-3 (>ninefold). Interestingly, vanadium exposure induced proteolytic cleavage of native protein kinase Cdelta $(\mathrm{PKC} \delta, 72-$ $74 \mathrm{kDa}$ ) to yield a $41 \mathrm{kDa}$ catalytically active fragment resulting in a persistent increase in PKC $\delta$ kinase activity. Co-treatment with pan-caspase inhibitor Z-VAD-FMK significantly blocked vanadium-induced $\mathrm{PKC} \delta$ proteolytic activation, indicating that caspases mediate PKC $\delta$ cleavage. Also, co-treatment with Z-VAD-FMK almost completely inhibited $\mathrm{V}_{2} \mathrm{O}_{5^{-}}$ induced DNA fragmentation. Furthermore, PKC $\delta$ knockdown using siRNA protected N27 cells from $\mathrm{V}_{2} \mathrm{O}_{5}$-induced apoptotic cell death. Collectively, these results demonstrate vanadium can exert neurotoxic effects in dopaminergic neuronal cells via caspase-3-
\end{abstract}


dependent PKC $\delta$ cleavage, suggesting that metal exposure may promote nigral dopaminergic degeneration.

KeyWords: metal mixtures, vanadium, manganese, neurotoxicity, oxidative stress, Parkinson's disease

\section{$\underline{\text { Introduction }}$}

Parkinson's disease (PD) is a multifactorial chronic neurodegenerative disorder associated with progressive degeneration of nigral dopaminergic neurons in the mesencephalic midbrain region resulting in substantial loss of dopaminergic neurotransmission to the striatal region (Anglade et al., 1997). The etiopathogenesis of PD is still poorly understood; however, chronic exposure to certain metals such as manganese (Mn) has been implicated in PD pathogenesis (Dobson et al., 2004). Epidemiological and casecontrol studies conducted in the United States as well as other countries have linked heavy metal exposure to an increased incidence of PD (Gorell et al., 1997; Fleming, 1994; Schulte, 1996; Liou, 1997; Marder, 1998; Smargiassi, 1998; Taylor, 1999; Priyadarshi, 2000; Tuchsen, 2000; Ritz, 2000). Subtle preclinical neurological effects have recently been documented following exposure to very low levels of Mn in occupational settings (Mergler, 1999; Crossgrove and Zheng, 2004). Since Mn elimination from the central nervous system (CNS) typically occurs very slowly, delayed neurotoxic effects may occur later in life resulting in an increased frequency of Parkinsonian diseases in the geriatric population (Alessio and Lucchini, 1996; Cranmer et al., 1999; Lucchini et al., 1995; Lucchini et al., 
1997). Early life exposure to heavy metals such as lead has been shown to produce Alzheimer's-like pathology in rodents as well as in primate models (Wu et al., 2008). Two studies have shown that welders are at an increased risk for the development of PD (Racette et al., 2001; Park et al., 2005), while another study did not find such an association between welding and a risk for developing Parkinsonism (Goldman et al., 2005; Ellingsen et al., 2008). Welding fumes contain many different metals including manganese, iron, and vanadium, typically present as vanadium pentoxide $\left(\mathrm{V}_{2} \mathrm{O}_{5}\right)$. However, the neurotoxic effects of $\mathrm{V}_{2} \mathrm{O}_{5}$ are not well understood.

Vanadium continues to be widely used in various industrial applications including steelmaking; arc welding; temperature-resistant alloy production; and glass, pigment and paint manufacturing (Hazardous Substance Database, ChemIDPlus, 2006; Bunting, 2006; McNeilly et al., 2004). Vanadium is a preferred metal for the production of special steels and temperature-resistant alloys because it is one of the lightest high-strength metals. More than $90 \%$ of industrial vanadium is used in steel making. The dominant market driver for vanadium over the past three years has been an increased worldwide demand for higher strength steel, most notably in China (Bunting, 2006). This increased demand for vanadium is not expected to decline as the worldwide demand for high quality steel continues. Welding and the associated exposure of workers to welding fumes have increased along with steel production. Among nine metals $(\mathrm{Co}, \mathrm{Cr}, \mathrm{Cu}, \mathrm{Fe}, \mathrm{Mn}, \mathrm{Ni}, \mathrm{Ti}, \mathrm{V}$ and $\mathrm{Zn})$ characterized in welding fumes by use of inductively coupled plasma mass spectroscopy (ICP-MS), vanadium was present at about half the concentration of Mn (McNeilly et al., 2004). The use of vanadium with non-ferrous metals is of particular importance in the atomic energy industry, aircraft construction and space technology (Hazardous Substance Database, ChemIDPlus, 
2006). Notably, vanadium compounds are released into the environment in large quantities, mainly by burning fossil fuels. Vanadium is usually found to be the most abundant trace metal in petroleum samples and can be found in concentrations reaching $1500 \mathrm{mg} \mathrm{kg}^{-1}$ depending on the source of the crude oil (Amorim et al., 2007). Vanadium accumulates in the soil, groundwater, and plants that may be consumed by animals and humans (Pyrzynska and Weirzbicki, 2004).

Despite these widespread use of vanadium, the health effects of the metal, in particular the CNS effects, are not well characterized. While earlier studies have shown vanadium exposure in humans may cause CNS deparession, tremor, neurasthesia and other severe motor deficits including vegetative symptoms (WHO, 2000; Done, 1979), the neurotoxic effects of vanadium and its potential to induce chronic neurological diseases are not well understood. Another recent study showed that inhaled $\mathrm{V}_{2} \mathrm{O}_{5}$ can damage the nigrostriatal dopaminergic system in rodent models (Avila-Costa et al., 2004), but the mechanism of vanadium-induced dopaminergic neurotoxicity is yet to be defined.

Oxidative stress and apoptosis are regarded as key mediators of neurodegenerative processes in PD (Hartmann et al., 2000; Dawson and Dawson, 1996; Olanow et al., 2004; Olanow and Tatton, 1999; Olanow, 2004) and are neurotoxic sequelae resulting from metal exposure (Kanthasamy et al., 2003; Kitazawa et al., 2003; Hamai and Bondy, 2004; Latchoumycandane et al., 2005). Our lab previously reported that increased oxidative stress during exposure to Parkinsonian neurotoxicants, as well as pesticides and metals, can activate the proapoptotic kinase $\mathrm{PKC} \delta$ by caspase-3-dependent proteolysis in cell culture models of PD (Kitazawa et al., 2003; Kaul et al., 2003, 2005(a), 2005(b); Latchoumycandane et al., 2005). Proteolytic cleavage of PKC $\delta(74 \mathrm{kDa})$ by caspase- 3 results in a $41 \mathrm{kDa}$ catalytic 
subunit and a $38 \mathrm{kDa}$ regulatory subunit, leading to a persistent activation of the kinase (Kaul et al., 2003; Kitazawa et al., 2003; Anantharam et al., 2004; Yang et al., 2004). Blockade of proteolytic activation of $\mathrm{PKC} \delta$ by overexpression of the kinase-dominant negative mutant, cleavage-resistant mutant, or siRNA directed against PKC $\delta$ almost completely prevented dopaminergic cell death (Kaul et al., 2003; Kitazawa et al., 2003, 2005; Anantharam et al., 2004; Yang et al., 2004), demonstrating that PKC $\delta$ is a key proapoptotic and oxidative stress sensitive kinase in dopaminergic neurons. In the present study, we examined the effect of $\mathrm{V}_{2} \mathrm{O}_{5}$ on oxidative signaling in a dopaminergic cell model of PD.

\section{$\underline{\text { Methods }}$}

\section{Chemicals}

Vanadium pentoxide $\left(\mathrm{V}_{2} \mathrm{O}_{5}\right)$ and MTT were purchased from Sigma (St. Louis, MO); Sytox green nucleic dye and COX IV antibody were purchased from Molecular Probes (Eugene, OR). Ac-DEVDAFC (Acetyl-Asp-Glu-Val-Asp-7-amido-4trifluoromethylcoumarin), Ac-LEHD-AFC (Acetyl- Leu-Glu-His-Asp-7-amido-4trifluoromethylcoumarin), and Z-VAD-FMK (Z-Val-Ala-Asp-fluoromethyl ketone) were purchased from MP Biomedicals (Aurora, OH). Cell Death Detection ELISA plus Assay Kit was purchased from Roche Molecular Biochemicals (Indianapolis, IN). Bradford protein assay kit was purchased from Bio-Rad Laboratories (Hercules, CA). RPMI 1640, B27 supplement, fetal bovine serum, L-glutamine, penicillin, and streptomycin were purchased from Invitrogen (Gaithersburg, MD). Nitric acid was purchased from Fisher Scientific 
(Pittsburgh, PA). Anti-mouse DMT-1 (1 $\mu \mathrm{g} / \mathrm{ml})$ and anti-mouse transferrin (Tf) $(1 \mu \mathrm{g} / \mathrm{ml})$ were purchased from Alpha Diagnostic International, San Antonio, TX. Protease cocktail, phosphatase inhibitors, ATP, Protein A-Sepharose, protein-G-Sepharose and anti- $\beta$-actin antibody were obtained from Sigma-Aldrich (St. Louis, MO); rabbit PKC $\delta$ antibody was purchased from Santa Cruz Biotechnology, Inc. (Santa Cruz, CA); cytochrome c antibody was purchased from BD Biosciences, anti-mouse and anti-rabbit secondary antibodies (Alexa Flour 680 conjugated anti-mouse IgG and Rabbit IgG IR800 Conjugate) were purchased from Invitrogen and Rockland Inc., respectively. $\left[\gamma^{32} \mathrm{P}\right] \mathrm{ATP}$ and ${ }^{3} \mathrm{H}-\mathrm{DA}$ were purchased from Perkin Elmer Life Science (Boston, MA).

\section{Cell culture}

We used the rat mesencephalic dopaminergic cell line referred to as N27 cells, which was a gift from Dr. Kedar N. Prasad (University of Colorado Health Sciences Center, Denver, CO). N27 cells have been used extensively to study the neurotoxic mechanisms pertaining to Parkinson's disease (Clarkson et al., 1999; Kaul et al., 2003; Kaul et al., 2005a; Kaul et al., 2005b; Miranda et al., 2004; Peng et al., 2005). N27 cells were grown and treated in RPMI 1640 medium supplemented with $10 \%$ fetal bovine serum, 2 mM Lglutamine, 50 units of penicillin, and $50 \mu \mathrm{g} / \mathrm{ml}$ of streptomycin in a humidified atmosphere of $5 \% \mathrm{CO}_{2}$ at $37^{\circ} \mathrm{C}$ as described previously (Kaul et al., 2003; Yang et al., 2004). For antioxidant studies, N27 cells were treated in RPMI 1640 medium supplemented with $2 \%$ B27 supplement with or without antioxidants instead of $10 \%$ fetal bovine serum. 
We also used mouse fetal primary mesencephalic cultures to determine the effect of vanadium on dopaminergic neurons. We prepared nigral primary mesencephalic neuronal cultures from the ventral mesencephalon of gestational 14-d-old mice embryos as described previously (Yang et al., 2004, Zhang et al., 2007). The mesencephalic tissues from mice were dissected, maintained in ice-cold $\mathrm{Ca}^{2+}$-free HBSS and then dissociated in HBSS solution containing trypsin-EDTA $(0.25 \%)$ for $20 \mathrm{~min}$ at $37^{\circ} \mathrm{C}$. The dissociated cells were then plated at equal density $\left(0.5 \times 10^{6}\right.$ cells $)$ in 30 -mm-diameter tissue culture wells which had been precoated with poly-D-lysine $(1 \mathrm{mg} / \mathrm{ml})$. The primary cultures were maintained in a chemically defined medium consisting of neurobasal medium fortified with B-27 supplements, L-glutamine $(500 \mu \mathrm{M})$, penicillin $(100 \mathrm{IU} / \mathrm{ml})$, and streptomycin $(100 \mu \mathrm{g} / \mathrm{ml})$ (Invitrogen). The cells were maintained in a humidified $\mathrm{CO}_{2}$ incubator $\left(5 \% \mathrm{CO}_{2}, 37^{\circ} \mathrm{C}\right)$ and half of the culture medium was replaced every $2 \mathrm{~d}$. Approximately 5- to 7-d-old cultures were used for experiments.

\section{Treatment paradigm}

For the purpose of this study, vanadium interchangeably refers to vanadium pentoxide $\left(\mathrm{V}_{2} \mathrm{O}_{5}\right)$ dissolved in water (moles of $\left.\mathrm{V}_{2} \mathrm{O}_{5} / \mathrm{L}\right)$. N27 cells were treated with different concentrations of vanadium for the duration of the experiments using diluted stock solution in culture media. After treatment, cells were collected by trypsinization or scraping, spun down at $200 \mathrm{~g}$ for $5 \mathrm{~min}$, and washed with ice-cold phosphate-buffered saline (PBS). The lysates from the cell pellets were used for various assays including caspase-3 activity, Western blotting, and measurement of DNA fragmentation. 


\section{Assessment of cell death by Sytox Green assay}

The assessment of cytotoxicity was conducted using Sytox Green, a membraneimpermeable DNA dye that enters dead cells as a result of altered membrane permeability and intercalates into the nucleic acid, as described previously (Kaul et al., 2005; Latchoumycandane et al., 2005). DNA-bound Sytox Green can be detected at an excitation wavelength of $485 \mathrm{~nm}$ and an emission wavelength of $538 \mathrm{~nm}$ using a fluorescence microplate reader (Bio-Tek microplate reader). The intensity of fluorescence is directly proportional to the number of dead cells; this method is known to be more efficient and sensitive than other cytotoxicity measurements (Kitazawa et al., 2004). Equal numbers of subconfluent N27 cells grown in 24-well plates were co-incubated with $1 \mu \mathrm{M}$ Sytox Green and with appropriate concentrations of vanadium or RPMI medium as a control. To quantify cell death, fluorescence intensity was monitored after the experiments were conducted and fluorescence pictures were taken using a Nikon inverted fluorescence microscope equipped with a SPOT digital camera (Diagnostic Instruments, Sterling Heights, MI).

\section{3-(4,5-dimethylthiazol-3-yl)-2,5-diphenyl tetrazolium bromide (MTT) assay}

This assay has been widely used to assess cell viability by measuring the activity of mitochondrial dehydrogenase enzymes that cleave the tetrazolium ring to produce formazan (Brown et al., 1994; Kitazawa et al., 2001; Choi et al., 2007). After vanadium treatment, cells were washed once and further incubated in serum-free DMEM containing $0.25 \mathrm{mg} / \mathrm{ml}$ MTT for $1 \mathrm{~h}$ at $37^{\circ} \mathrm{C}$. Supernatant was removed, and MTT crystals were solubilized in $200 \mu \mathrm{l}$ dimethyl sulfoxide. The mitochondrial activity was measured with the SpectraMax 
spectrophotometer (Molecular Devices Corporation, San Diego, CA) at $570 \mathrm{~nm}$, with the reference wavelength at $630 \mathrm{~nm}$.

\section{Determination of intracellular vanadium concentration}

$\mathrm{N} 27$ cells were treated with $40 \mu \mathrm{M}$ vanadium for $0,4.5$ and $9 \mathrm{~h}$ and washed three times with PBS. Inductively coupled plasma mass spectrometry (ICP-MS) was used to determine the concentrations of $\mathrm{V}$ at $\mathrm{m} / \mathrm{z} 51$ in each sample. The ICP-MS device (ELEMENT 1, Thermo Finnigan) was a high-resolution double focusing instrument operated in medium resolution $(\mathrm{m} / \Delta \mathrm{m}=4,000)$ in order to resolve the isotopes of interest from any interferences (Shum et al., 1992). Each sample was placed in an acid-washed $5 \mathrm{ml}$ Teflon vial and digested in $150 \mu \mathrm{l}$ high purity nitric acid (Ultrex II, J.T. Baker). Following digestion, the samples were diluted to $5 \mathrm{ml}$ with $18.2 \mathrm{M} \Omega$ deionized water to give a final acid concentration of approximately $3 \%$ nitric acid. The supernatant was analyzed with the ICPMS.

An internal standard method was used for quantification. Gallium was chosen as the internal standard because its $\mathrm{m} / \mathrm{z}$ ratio is similar to that of the elements of interest, and it has no major spectroscopic interferences. A small spike of Ga standard solution was added to each sample for a final Ga concentration of $10 \mathrm{ppb}$. A $10 \mathrm{ppb}$ multi-element standard (V, $\mathrm{Mn}, \mathrm{Fe}, \mathrm{Cu}, \mathrm{Zn}, \mathrm{Ga}$ ) was prepared. The nitric acid blank, the multi-element standard, and each of the samples were introduced into the ICP-MS via a $100 \mu \mathrm{l} / \mathrm{min}$ self-aspirating PFA nebulizer (Elemental Scientific, Inc.). The nitric acid blank was used to rinse the nebulizer between each sample. 
The results for each sample were calculated using the integrated average backgroundsubtracted peak intensities from 20 consecutive scans. To correct for differences in elemental ionization efficiency in the ICP, the multi-element standard was used to derive normalization factors for $\mathrm{V}, \mathrm{Mn}$ and $\mathrm{Cu}$. Concentrations for $\mathrm{V}, \mathrm{Mn}$ and $\mathrm{Cu}$ were then calculated for each sample.

\section{Determination of vanadium-induced $\mathrm{H}_{2} \mathrm{O}_{2}$ production by polarography}

N27 cells ( 10,000 per well) were grown in 96 well plates $12-18 \mathrm{~h}$ prior to treatments and then exposed to vanadium under serum free conditions for $4 \mathrm{~h} . \mathrm{H}_{2} \mathrm{O}_{2}$ production in N27 cells was measured using an Apollo 4000 Free Radical Analyzer (WPI, Sarasota, FL) equipped with a $100-\mu \mathrm{m} \mathrm{H}_{2} \mathrm{O}_{2}$ sensor following a $4 \mathrm{~h}$ vanadium exposure. Before the experiment, the electrode was calibrated using serial dilutions of $\mathrm{H}_{2} \mathrm{O}_{2}$, and the current recorded from the serum free RPMI media over the cells was then calculated as concentration of $\mathrm{H}_{2} \mathrm{O}_{2}$. The APOLLO 4000 Free-Radical Analyzer (World Precision Instruments, Inc., Sarasota, FL, USA), which is increasingly being used (Mastore et al., 2005; Castello et al., 2007), was used to monitor in real-time the production of $\mathrm{H}_{2} \mathrm{O}_{2}$ during vanadium treatment. A pulse voltage $(+400 \mathrm{mV})$ maintained on a sensitive and selective $\mathrm{H}_{2} \mathrm{O}_{2}$ sensor (ISO-HOP2) ensured that the electrochemical responses (redox current) generated at the working electrode were derived only from the oxidation of any $\mathrm{H}_{2} \mathrm{O}_{2}$ formed, and that these responses were proportional to the concentration of the reactive molecule. Quantitative determinations were made following the establishment of calibration curves for the $\mathrm{H}_{2} \mathrm{O}_{2}$ electrode prior to all tests. The latter was obtained by plotting changes in current (pA) against changes in $\mathrm{H}_{2} \mathrm{O}_{2}$ concentration. Test conditions, such as temperature and 
$\mathrm{pH}$, were identical to those under which the instrument was calibrated (Mastore et al., 2005). To assess $\mathrm{H}_{2} \mathrm{O}_{2}$ production, the electrode was allowed to equilibrate for 1-3 min in treatment media on cells in the wells of the 96 well plate.

\section{Measurement of caspase-3 and caspase-9}

Caspase-3 and caspase-9 activities were measured as previously described in our lab publications (Anantharam et al., 2002; Kaul et al., 2003; Kitazawa et al., 2003; Kitazawa et al., 2002). After exposure to vanadium, the cells were washed with PBS, resuspended in lysis buffer containing $50 \mathrm{mM}$ Tris/HCl (pH 7.4), 1 mM EDTA, 10 mM EGTA, and $10 \mu \mathrm{M}$ digitonin, and incubated at $37^{\circ} \mathrm{C}$ for $20 \mathrm{~min}$. Lysates were centrifuged at $132,000 \mathrm{~g}$, and the cell-free supernatants were incubated with $50 \mu \mathrm{M}$ Ac-DEVD-AFC (fluorometric caspase-3 substrate) or $50 \mu \mathrm{M}$ Ac-LEHD-AFC (fluorometric caspase-9 substrate) at $37^{\circ} \mathrm{C}$ for $1 \mathrm{~h}$. Formation of 7-amido-4-trifluoromethylcoumarin (AFC) resulting from caspase cleavage was measured using a fluorescence plate reader (excitation $400 \mathrm{~nm}$, emission $505 \mathrm{~nm}$ ). All the fluorescence signals from the samples were normalized to protein concentration, as determined with the Bradford protein assay.

\section{DNA fragmentation assay}

DNA fragmentation assays were performed using a Cell Death Detection ELISA plus Assay Kit, which is fast, highly sensitive and reliable for the detection of early changes in cells undergoing apoptotic cell death. The assay analyzed DNA fragmentation by quantification of histone-associated low molecular weight DNA in the cytoplasm of cells (Anantharam et al., 2002; Kaul et al., 2003; Kitazawa et al., 2002). DNA fragmentation was 
measured in N27 cells exposed to vanadium at time points correlating with maximum caspase-3 activation. In inhibitor studies, pan-caspase inhibitor Z-VAD-FMK (100 $\mu \mathrm{M})$ was co-treated (after a 30 min pretreatment with the inhibitors alone) with $40 \mu \mathrm{M}$ vanadium for 9 h. After treatment, $20 \mu \mathrm{l}$ of cell lysate was prepared according to the manufacturer's protocol, as previously described (Choi et al., 2007). Briefly, vanadium-treated cells were washed with PBS, and the cell pellets were then resuspended with the lysis buffer provided in the assay kit. The lysate was spun down at $200 g$, and $20 \mu 1$ of supernatant was incubated for $2 \mathrm{~h}$ with the mixture of HRP-conjugated antibody that recognizes histones and single- and doublestranded DNA. After washing away the unbound components, the final reaction product was measured colorimetrically with 2,2'-azino-di-[3-ethylbenz-thiazoline sulfonate] as an HRP substrate using a spectrophotometer at $405 \mathrm{~nm}$ and $490 \mathrm{~nm}$. The difference in absorbance between 405 and $490 \mathrm{~nm}$ was used to determine the amount of DNA fragmentation in each sample. All sample concentrations were normalized to protein concentration using the Bradford protein assay.

\section{PKCo knockdown by siRNA in N27 cells}

PKC $\delta$-siRNA was prepared by an in vitro transcription method, as described previously (Yang et al., 2004). Initially, siRNA target sites specific to rat PKC $\delta$ mRNA (gene identifier: 18959249), as determined by blast analysis, were chosen. One nonspecific siRNA (NS-siRNA) was also chosen based on random sequence. For each siRNA, sense and antisense templates were designed based on each target sequence and partial T7 promoter sequence (Donze and Picard, 2002): for PKC $\delta$-siRNA, sense, 5'AACTGTTTGTGAATTTGCCTTCCTGT CTC-3'; antisense, 5'- 
AAAAGGCAAATTCACAAACAGCCTGTCTC-3' with the target site located at nucleotide 2142 to 2162 in rat PKC $\delta$ mRNA with a GC content of 47.6\%; for NS-siRNA, sense, 5'AATTCTCACACTTCGGAGAACCTGTCTC-3'; antisense, 5'-AAGTTCTCCG AAGTGTGAGAACCTGTCTC-3'. All template oligonucleotides were chemically synthesized and PAGE purified. In vitro transcription, annealing, and purification of siRNA duplexes were performed using the protocol supplied with the silencer siRNA construction kit (Ambion, Austin, TX). Briefly, $\sim 2 \mu \mathrm{g}$ of each single-strand (ss) transcription template was first annealed with the $\mathrm{T} 7$ promoter and filled in by Klenow DNA polymerase to form double-strand transcription templates. For preparation of each siRNA duplex, transcription reactions were first performed with separated antisense and sense templates using the T7 RNA polymerase provided with the kit and then annealed to form siRNA duplexes. The siRNA duplex was then treated with DNase and RNase to remove the extra nucleotides of transcribed siRNA to meet the structural 3'UU overhang and 5' phosphate requirement (Elbashir et al., 2001). Previously, we showed that PKC $\delta$-siRNA effectively suppresses $>80 \%$ of $\mathrm{PKC} \delta$ protein expression levels within $24 \mathrm{~h}$ post-transfection (Yang et al., 2004). N27 cells (50-70\% confluence) were transfected with siRNA duplexes by using an AMAXA Nucleofector kit (AMAXA), as described in our lab's previous study (Yang et al., 2004).

\section{Protein kinase $\mathbf{C} \delta$ activity}

PKC $\delta$ enzymatic activity was determined using immunoprecipitation, as described previously (Kitazawa et al., 2003; Kaul et al., 2005). The cells were exposed to $40 \mu \mathrm{M}$ vanadium for $9 \mathrm{~h}$, with or without a pan-caspase inhibitor (Z-VAD-FMK), and cell lysates were collected. After immunoprecipitation with anti-PKC $\delta$ antibody, $25 \mu 1$ samples 
containing PKC $\delta$ bound to Sepharose-A beads were incubated with $25 \mu 1$ of reaction buffer containing $0.4 \mathrm{mg}$ of histone $\mathrm{H} 1$ and $5 \mu \mathrm{Ci}$ of $\left[\gamma_{-}{ }^{32} \mathrm{P}\right] \mathrm{ATP}(4500 \mathrm{Ci} / \mathrm{mM})$ for $10 \mathrm{~min}$ at $30^{\circ} \mathrm{C}$. The reaction was terminated by the addition of 2x SDS gel loading buffer and boiled for 5 min. The samples were separated on $12 \%$ SDS-PAGE and histone phosphorylated bands were detected using a PhosphoImager (Personal Molecular Imager FX, Bio-Rad) and quantified using Quantity One 4.2.0 Software (Bio-Rad).

\section{Western blotting}

$\mathrm{N} 27$ neuronal cells were exposed to $40 \mu \mathrm{M} \mathrm{V}_{2} \mathrm{O}_{5}$ with or without the pan-caspase inhibitor (Z-VAD-FMK) at $37^{\circ} \mathrm{C}$ for appropriate time points. $\mathrm{N} 27$ cells were lysed, homogenized, sonicated, and centrifuged as described previously (Kaul et al., 2003; Kitazawa et al., 2002). The supernatants were collected as cell lysates, and protein concentrations were determined and used for SDS-gel electrophoresis according to standard procedure. Whole cell lysates and cytoplasmic and mitochondrial fractions, as appropriate, containing equal amounts of protein were loaded in each lane and separated on a 10-15\% SDS-PAGE gel, as described previously (Kaul et al., 2003; Kitazawa et al., 2002). Proteins were then transferred to nitrocellulose membrane, and non-specific binding sites were blocked by incubation for $1 \mathrm{~h}$ in Licor buffer. The membranes were then treated with the appropriate primary antibodies, $\mathrm{PKC} \delta$ polyclonal antibody (1:2000), cytochrome c (1:500), COX IV(1:500), Tf (1:500), and DMT1 (1:500) followed by treatment with secondary antimouse or anti-rabbit antibodies, as appropriate. To confirm equal protein in each lane, membranes were probed with $\beta$-actin antibody (1:5000 dilution). Western blot was performed using IR dye-800 conjugated anti-rabbit dye and Alexa Flour 680 conjugated anti-mouse IgG 
as secondary antibodies. Western blot images were captured and analyzed with an Odyssey IR Imaging system (LICOR).

\section{Dopaminergic Neuronal Viability by ${ }^{3}$ H-Dopamine $\left({ }^{3}\right.$ H-DA) Uptake Assay}

The neurotoxic effect of $\mathrm{V}_{2} \mathrm{O}_{5}$ on dopaminergic neurons in fetal mouse mesencephalic cultures were quantified using ${ }^{3} \mathrm{H}$-DA uptake assay described in detail elsewhere (Michel et al., 1990; Vaglini et al., 2008). In our experience, we noted that ${ }^{3} \mathrm{H}-\mathrm{DA}$ uptake assay was more robust and quantitative than tyrosine hydroxylase (TH) positive cell counts by immunohistochemical method. Briefly, mouse primary mesencephalic neuronal cultures were treated with $10,20,40$ or $60 \mu \mathrm{M} \mathrm{V}_{2} \mathrm{O}_{5}$ for $12 \mathrm{~h}$ and then cells were washed once with assay incubation (Kreb's Ringer) buffer (5.6mM glucose, 1.3mM EDTA, 1.2mM magnesium sulfate, $1.8 \mathrm{mM}$ calcium chloride, $4.7 \mathrm{mM}$ potassium chloride, $120 \mathrm{mM}$ sodium chloride, $16 \mathrm{mM}$ sodium phosphate). Cells were incubated with $10 \mu \mathrm{M}{ }^{3} \mathrm{H}-\mathrm{DA}(30 \mathrm{Ci} / \mathrm{mol})$ for $30 \mathrm{~min}$ at $37^{\circ} \mathrm{C}$. The dopamine reuptake blocker Mazindol $(1 \mathrm{nM})$ was used as a positive control to assess the efficiency of ${ }^{3} \mathrm{H}-\mathrm{DA}$ uptake. The uptake was stopped by removing the reaction mixture and followed by a three-time wash with fresh Kreb's Ringer buffer. Cells were then collected with $1 \mathrm{~N}$ sodium hydroxide and the radioactivity was measured by liquid scintillation counter after the addition of a $4 \mathrm{~mL}$ scintillation cocktail to each vial.

\section{Statistical analysis}

Data were analyzed with Prism 3.0 software (GraphPad Software, San Diego, CA). Tukey's multiple comparison testing was used to compare differences between treatment groups of N27 cells. For the cell viability assay, a nonlinear regression curve was fit onto the 
data, and $\mathrm{EC}_{50}$ concentrations were extrapolated (Choi et al., 2007). For comparison between two samples, Student's T-test was performed to examine the differences. Differences with * $p<0.05, * * p<0.01$, and $* * * p<0.001$ were considered significant and are indicated by asterisks. Presented data typically represent results from at least two separate experiments with triplicate samples where appropriate, and are expressed as mean \pm S.E.M.

\section{$\underline{\text { Results }}$}

\section{Vanadium exposure induces dose-dependent increase in cytotoxicity}

In order to determine the optimal dose for a detailed mechanistic investigation of vanadium neurotoxicity, we first performed a dose-response cytotoxicity analysis. N27 cells were exposed to 3-300 $\mu \mathrm{M}$ vanadium $\left(\mathrm{V}_{2} \mathrm{O}_{5}\right)$ for $12 \mathrm{~h}$, and a dose-dependent effect of vanadium on cytotoxic cell death was determined by MTT assay. As shown in Fig. 1A, a dose-dependent decrease in cell viability was observed. $\mathrm{An} \mathrm{EC}_{50}$ of $37 \pm 3.47 \mu \mathrm{M}$ for vanadium was deduced by three-parameter nonlinear regression from the dose-response curve. The dose-dependent effect of vanadium-induced cell death was further confirmed by Sytox Green fluorescence assay, which labels green only dead/dying cells both qualitatively and quantitatively. N27 cells were exposed to $10-300 \mu \mathrm{M}$ vanadium for $12 \mathrm{~h}$. Fig. 1B is representative of untreated and vanadium-treated N27 cells at the end of a $12 \mathrm{~h}$ treatment in phase-contrast (left panels) and Sytox FITC fluorescence imaging (right panels). An increase in the number of Sytox-positive green cells indicates an increase in cell death because the Sytox Green dye permeates compromised cell membranes to stain nuclear chromatin. The 
number of Sytox-positive cells increased dose-dependently in vanadium-treated cells compared to untreated controls. Quantitative analysis of Sytox fluorescence using a fluorescence plate reader also revealed that vanadium treatment induced cytotoxic cell death in N27 cells. As shown in Fig. 1C, vanadium increased cell death in a dose-dependent manner. Exposure to $10,30,100$ and $300 \mu \mathrm{M}$ vanadium over $12 \mathrm{~h}$ resulted in a one to sixfold increase in the number of Sytox-positive cells compared to untreated control cells.

\section{Time-dependent uptake of vanadium in N27 dopaminergic cells}

Since vanadium induced cytotoxic cell death in a dose-dependent manner, we measured the amount of vanadium entering the cell over time. Previously, we and others have shown that metal ions, $\mathrm{Mn}, \mathrm{Fe}, \mathrm{Zn}, \mathrm{Cu}, \mathrm{Co}, \mathrm{Cd}, \mathrm{Al}$, and $\mathrm{V}$, can be transported by $\mathrm{Tf}$ and divalent metal ion transporter (DMT1) (Aschner and Aschner, 1991; Choi et al., 2007; Erikson et al., 2004). Using ICP-MS, we observed a time-dependent uptake of vanadium (Fig. 2A). Exposure to $40 \mu \mathrm{M}$ vanadium to N27 cells resulted in a 7- and 11-fold increase in intracellular levels of vanadium following exposure for 4.5 and $9 \mathrm{~h}$, respectively. In addition, we also observed a significant upregulation of transport proteins Tf (Fig. 2B) and DMT1 (Fig. 2C) over time, suggesting that these transport proteins may play a role in the time-dependent uptake of vanadium by the dopaminergic neurons. We also found vanadium treatment altered concentrations of other essential metals including $\mathrm{Mn}$ and $\mathrm{Cu}$ in the cells. As shown in Fig 2D, intracellular Mn increased by 1.4-fold at $4.5 \mathrm{~h}$ of $40 \mu \mathrm{M} \mathrm{V}_{2} \mathrm{O}_{5}$ treatment whereas the level decreased by 2.7 -fold at longer time $\mathrm{V}_{2} \mathrm{O}_{5}$ exposure (Fig 2D). The intracellular copper increased by 3 - and 3.7-fold at $4.5 \mathrm{~h}$ and $9 \mathrm{~h}$, respectively, following $\mathrm{V}_{2} \mathrm{O}_{5}$ exposure (Fig 2E). 


\section{Vanadium induces oxidative stress in N27 mesencephalic neuronal cells}

Based on the cytotoxicity data and uptake studies, we chose an optimal dose of 40

$\mu \mathrm{M}$ vanadium $\left(\mathrm{V}_{2} \mathrm{O}_{5}\right)$ for all subsequent experiments. Several studies, including ours, have shown that dopaminergic neurotoxicants including MPP+, Mn, dieldrin, and MMT induce oxidative stress, alter mitochondrial function and mediate the release of a number of proapoptotic factors including cytochrome c into the cytosol (Kitazawa et al., 2001; Anantharam et al., 2002; Kaul et al., 2003; Kanthasamy et al., 2005) to initiate the apoptotic cascade. Therefore, we examined whether vanadium exposure induces ROS production in dopaminergic cells. In this experiment, we used a very sensitive APOLLO 4000 FreeRadical Analyzer for measurement of $\mathrm{H}_{2} \mathrm{O}_{2}$ generation. Fig. 3A shows the standard curve for $\mathrm{H}_{2} \mathrm{O}_{2}$ with $\mathrm{R}^{2}$ 0.999. N27 cells were exposed to $40 \mu \mathrm{M}$ vanadium for $4 \mathrm{~h}$ and then $\mathrm{H}_{2} \mathrm{O}_{2}$ levels were measured. As shown in Fig. 3B, vanadium exposure induced a threefold significant increase in $\mathrm{H}_{2} \mathrm{O}_{2}$ production compared with the control, indicating that vanadium can promote oxidative stress in dopaminergic cells.

Next we examined whether vanadium-induced oxidative stress plays a role in the neurotoxicity of vanadium. An antioxidant cocktail (AO) consisting of vitamin E, glutathione, superoxide dismutase (SOD), and catalase was used in the study. N27 cells were exposed to $40 \mu \mathrm{M}$ vanadium with or without an $\mathrm{AO}$ for $12 \mathrm{~h}$ and then cytotoxicity was measured by Sytox green fluorescence assay. Co-treatment with AO almost completely protected vanadium-induced neurotoxicity, as seen in phase-contrast (Fig. 3C right panels) and Sytox green fluorescence imaging (Fig. 3C left panels). Quantitative analysis of Sytox fluorescence revealed that $\mathrm{AO}$ treatment blocks the threefold increase in neurotoxicity 
induced by vanadium. These results demonstrate that vanadium-induced oxidative stress plays a key role in mediating the neurotoxicity in dopaminergic cells.

\section{Vanadium exposure promotes mitochondrial cytochrome c release}

Previously we showed that exposure to dopaminergic neurotoxins alters mitochondrial function, which can result in the release of a number of proapoptotic factors, including cytochrome c, into the cytosol to initiate the apoptotic cascade in neuronal cells (Kitazawa et al., 2001; Anantharam et al., 2002; Kaul et al., 2003). In the present study, N27 cells were exposed to $40 \mu \mathrm{M}$ vanadium for 3 and $6 \mathrm{~h}$ and the release of cytochrome c into the cytosol was measured by Western blot analysis using antibodies directed against cytochrome c. As shown in Fig. 4, treatment of N27 cells with $40 \mu \mathrm{M}$ vanadium for 3 and 6 h resulted in a significant increase in cytosolic cytochrome c compared to untreated controls. The mitochondrial marker COX-IV (15-17 kDa) was used for testing the purity of the cytosolic fractions, and no mitochondrial contamination was noted. Nitrocellulose membranes were reprobed with $\beta$-actin antibody to confirm equal protein loading.

\section{Vanadium induces a time-dependent activation of caspase-9 and caspase-3}

Cytosolic cytochrome $\mathrm{c}$ release has been shown to activate multiple caspases, including caspase-9 and caspase-3 (Dawson and Dawson, 2003; Kanthasamy et al., 2003). Caspase-9 and caspase-3 play an important role in the execution of mitochondrial-dependent apoptotic cell death. Since vanadium increased cytochrome c release, we examined the effect of vanadium on the activities of caspase- 9 and caspase- 3 in N27 cells. Exposure to 40 $\mu \mathrm{M}$ vanadium induced time-dependent increases in caspase-9 (Fig. 5A) and caspase-3 (Fig. 
5B) activities compared to untreated controls. Relative to untreated control cells we observed greater than fourfold and ninefold increases in caspase activities for caspase- 9 and caspase-3, respectively. Furthermore, co-treatment with $100 \mu \mathrm{M} Z$-VAD-FMK, a pan-caspase inhibitor, significantly blocked $40 \mu \mathrm{M}$ vanadium-induced caspase-9 (Fig. 5C) and caspase-3 (Fig. 5D) enzymatic activities at a level nearly equal to untreated control levels, demonstrating the specificity of caspase activation during vanadium exposure.

\section{Caspase-3 mediates proteolytic cleavage of PKC $\delta$ in vanadium exposed N27 cells}

Recently, we demonstrated that PKC $\delta$ is a prominent endogenous substrate for caspase-3 in dopaminergic cells undergoing apoptotic cell death (Kikkawa et al., 2002; Brodie and Blumberg, 2003; Kanthasamy et al., 2003). Caspase-3 cleaves PKC $\delta$ to yield a $41 \mathrm{kDa}$ catalytically active subunit and a $38 \mathrm{kDa}$ regulatory subunit. Proteolytic cleavage of $\mathrm{PKC} \delta$ results in the permanent dissociation of the regulatory domain from the catalytic fragment, resulting in persistently active kinase. Previously, we showed that Mn and other dopaminergic toxicants induced proteolytic cleavage of $\mathrm{PKC} \delta$, but not of $\mathrm{PKC} \alpha, \beta$, or $\gamma$ in an isoform specific and caspase-3 dependent manner (Latchoumycandane et al., 2005). Since vanadium exposure resulted in caspase-3 activation in N27 cells (Fig. 5), we examined the proteolytic cleavage of $\mathrm{PKC} \delta$ in vanadium-treated N27 cells. A $9 \mathrm{~h}$ vanadium treatment induced PKC $\delta$ cleavage (Fig. 6A), while no cleavage of PKC $\delta$ was observed in untreated control cells. In subsequent experiments, we used the cell-permeable caspase inhibitor ZVAD-FMK to confirm that PKC $\delta$ cleavage is mediated by caspase-3. Co-treatment with 100 $\mu \mathrm{M}$ Z-VAD-FMK almost completely blocked vanadium-induced PKC $\delta$ cleavage (Fig. 6A), 
suggesting that the cleavage is indeed mediated by caspase-3. Nitrocellulose membranes were reprobed with $\beta$-actin antibody to confirm equal protein loading.

\section{Vanadium induces increases in $\mathrm{PKC} \delta$ kinase activity in a caspase-dependent manner}

To determine if the vanadium-induced $\mathrm{PKC} \delta$ cleavage also leads to an increase in PKC $\delta$ enzyme activity, we performed immunoprecipitation kinase assays by examining the ability of immunoprecipitated $\mathrm{PKC} \delta$ to phosphorylate histone $\mathrm{H} 1$ using $\left[{ }^{32} \mathrm{P}\right] \mathrm{ATP}$. We performed the kinase assay in the absence of lipids to measure the increased kinase activity resulting from the persistently active $\mathrm{PKC} \delta$ catalytic fragment due to proteolytic cleavage, but not by the activation of full-length $\mathrm{PKC} \delta$ due to membrane translocation. A $9 \mathrm{~h}$ treatment with $40 \mu \mathrm{M}$ vanadium in $\mathrm{N} 27$ dopaminergic cells induced a very significant increase in PKC $\delta$ kinase activity compared to the control (Fig. 6B). The vanadium-induced $\mathrm{PKC} \delta$ kinase activity was significantly attenuated in cells co-treated with $100 \mu \mathrm{M}$ of the pancaspase inhibitor Z-VAD-FMK. Exposure to $40 \mu \mathrm{M}$ vanadium for $9 \mathrm{~h}$ resulted in increases of $50 \%$ and $10 \%$ in PKC $\delta$ kinase activity in vanadium- and $\mathrm{V}_{2} \mathrm{O}_{5}+\mathrm{Z}-\mathrm{VAD}-\mathrm{FMK}$-treated samples, respectively, when compared to untreated control cells, as revealed by densitometric analysis of phosphorylated histone $\mathrm{H} 1$ bands (Fig. 6B). These results suggest that proteolytic cleavage of $\mathrm{PKC} \delta$ mediated by caspase- 9 and -3 increases the kinase activity.

\section{Caspases mediate vanadium-induced apoptotic and cytotoxic cell death in N27 cells}

Chromatin condensation and DNA fragmentation are hallmarks of apoptosis during metal neurotoxicity (Kanthasamy et al., 2003; 2005). To understand the functional 
consequence of activation of ROS production, cytochrome c release, and caspase- 9 , caspase3 and $\mathrm{PKC} \delta$ activation, we tested whether vanadium induces apoptosis by using a quantitative DNA fragmentation ELISA assay. Fig. 7A shows that the vanadium-induced DNA fragmentation in N27 cells is significantly attenuated in cells co-treated with the pancaspase inhibitor Z-VAD-FMK. Exposure to $40 \mu \mathrm{M}$ vanadium for $9 \mathrm{~h}$ caused a fourfold increase in DNA fragmentation compared to untreated control cells, while co-treatment with $100 \mu \mathrm{M}$ Z-VAD-FMK blocked vanadium-induced DNA fragmentation by more than $60 \%$. Further, we also show that Z-VAD-FMK suppressed vanadium-induced cytotoxic cell death, as measured by Sytox assay (Fig. 7B). Co-treatment with Z-VAD-FMK very significantly attenuated vanadium-induced increases in the number of Sytox-positive cells at the end of a $9 \mathrm{~h}$ treatment, as seen in phase-contrast (right panels) and Sytox FITC fluorescence imaging (left panels). Together, these data suggest that both caspases-9 and -3 contribute to vanadium-induced DNA fragmentation.

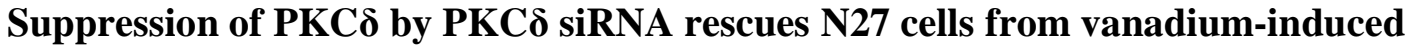 apoptotic cell death}

To further substantiate the functional role of PKC $\delta$ in vanadium-induced apoptotic cell death, we examined the effect of PKC $\delta$ siRNA on vanadium-induced DNA fragmentation. Our lab has developed PKC $\delta$ siRNAs that specifically suppress PKC $\delta$ expression without producing any cytotoxic effect in dopaminergic neurons (Yang et al., 2004). Western blot analysis revealed that the protein levels of PKC $\delta$ were significantly suppressed in $\mathrm{PKC} \delta$-siRNA-transfected cells, whereas $\mathrm{PKC} \delta$ expression levels were unaltered in non-specific-siRNA (siRNA-NS) or untreated control cells (Fig. 8A). We have 
shown in our lab that suppression of $\mathrm{PKC} \delta$ may regulate upstream caspases via a positive feedback amplification loop resulting in the persistent activation of the protein (Kaul, 2003; Kitazawa, 2003). Vanadium-induced caspase-3 activity was significantly blocked in PKC $\delta$ siRNA transfected N27 cells (Fig. 8B). Importantly, PKC $\delta$-siRNA knockdown almost completely blocked vanadium-induced DNA fragmentation, demonstrating a key proapoptotic function of PKC $\delta$ in vanadium-induced dopaminergic cell death.

\section{Vanadium induces neurotoxic responses to primary dopaminergic neurons}

To determine if vanadium was toxic to nigral dopaminergic neurons, we exposed mouse primary mesencephalic neuronal cultures to various doses of $\mathrm{V}_{2} \mathrm{O}_{5}$ for $12 \mathrm{~h}$ and then assessed the viability of dopaminergic neurons using ${ }^{3} \mathrm{H}-\mathrm{DA}$ assay. Mazindol, a dopamine reuptake inhibitor, was used as positive control for the assay. A 12-h treatment with 10, 20, 40 , and $60 \mu \mathrm{M}$ vanadium in nigral primary neuronal cells induced a dose-dependent decrease in dopamine uptake (Fig. 9), indicative of loss of nigral dopaminergic neurons. Forty and 60 $\mu \mathrm{M} \mathrm{V}_{2} \mathrm{O}_{5}$ produced a significant dopaminergic neuronal degeneration as compared to untreated control cells following at $12 \mathrm{~h}$ exposure. These results indicate that vanadium can induce a neurotoxic effect to dopaminergic neurons at low micromolar concentrations.

\section{$\underline{\text { Discussion }}$}

In this study, for the first time to the best of our knowledge, we demonstrate that vanadium can induce oxidative stress and apoptosis in mesencephalic dopamine-producing 
neuronal (N27) cells through the activation of a series of oxidative stress mediated specific cell death signaling events, including release of cytochrome $\mathrm{c}$ from the mitochondria into the cytosol, activation of caspase-9 and caspase-3, proteolytic cleavage of $\mathrm{PKC} \delta$, and nuclear DNA breakdown. Using the pharmacological pan-caspase inhibitor Z-VAD-FMK and siRNA against $\mathrm{PKC} \delta$, we established that $\mathrm{PKC} \delta$ is a key downstream mediator of an oxidative stresscaspase-mediated signaling cascade during vanadium-induced apoptosis in dopaminergic neuronal cells. Our results also show that metal transporter proteins Tf and DMT1 may play a key role in the time-dependent uptake and transport of vanadium in dopaminergic neuronal cells. The results from the primary mesencephalic cultures suggest that vanadium can induce degeneration of nigral dopaminergic neurons at low micromolar concentrations.

Acute exposure to vanadium pentoxide has been shown to have major pathophysiological manifestations on the nervous system in humans (WHO, 2000). Severe chronic exposure in humans produces CNS symptomatology including nervous disturbances and neurasthenic or vegetative symptoms (WHO, 2000). Another report showed that individuals exposed to vanadium manifest some neurological disorders such as tremor and CNS depression (Done, 1979). Despite these data on the potential neurotoxic effect of vanadium in humans, only very limited neurotoxicological studies are available to date. Inhalation of vanadium pentoxide in rodent models produces a time-dependent loss of dendritic spines, necrotic-like cell death and considerable alterations of the hippocampus CA1 neurophile, all of which are associated with spatial memory impairment (Avila-Costa et al., 2006). Within the ependymal epithelium, cilia loss, cell sloughing and cell layer detachment occur after vanadium pentoxide inhalation in rodents (Avila-Costa et al., 2005). 
This damage disrupts the permeability of the epithelium and promotes access of inflammatory mediators to the underlying neuronal tissue, causing injury and neuronal death (Avila-Costa et al., 2005). Results from a rat study provide evidence that postnatal vanadium exposure through lactation may have an adverse impact upon the physical and neural development of the offspring and on CNS myelination (Soazo and Graciela, 2007). A decreased number of tyrosine hydroxylase immunoreactive neurons in the substantia nigra pars compacta and loss of dendritic spine in the corpus striatum following exposure to vanadium were recently reported in a rat model (Avila-Costa et al., 2004). In the present study, we show that vanadium is neurotoxic in a dopaminergic neuronal model, with an $\mathrm{EC}_{50}$ of $37 \pm 3.47 \mu \mathrm{M}$. In comparison, the $\mathrm{EC}_{50}$ of manganese in the same dopaminergic neuronal model was $345 \mu \mathrm{M}$ (Latchoumycandane et al., 2005), indicating that vanadium is more toxic than manganese in dopaminergic neuronal cells. Cytotoxicity induced by vanadium compounds has been reported in non-neuronal cells (Cortizo et al., 2000; Sabbioni et al., 1991, 1981), and the toxicity of vanadium compounds increases as the valence increases (Barceloux, 1999).

Tf and DMT-1 are major metal transport proteins in CNS. Tf binds several metals including $\mathrm{Fe}, \mathrm{Mn}, \mathrm{Zn}$, and $\mathrm{Cr}$ as well as $\mathrm{Cu}, \mathrm{Co}, \mathrm{Cd}, \mathrm{V}$, and $\mathrm{Al}$ and mediates the transport of these metals (Aschner and Aschner, 1991). Another study showed that Mn and Fe are transported by Tf-dependent and -independent pathways, both involving DMT-1 as the transport protein (Erikson et al., 2004). In this study, we saw increased protein levels of Tf and DMT-1, suggesting that they play a role in the time-dependent uptake of vanadium by the dopaminergic neurons. Our results also suggest that the uptake of vanadium by 
dopaminergic neurons is accompanied by changes in the concentration of other essential metals such as $\mathrm{Mn}$ and $\mathrm{Cu}$. Future studies are needed to clarify the mechanisms of vanadium transport in the dopaminergic system.

We have previously shown that exposure to Mn and Mn-containing MMT leads to ROS generation as well as depolarization of the mitochondrial membrane potential in dopaminergic neuronal cells (Anantharam et al., 2002; Kitazawa et al., 2002). We also reported that dopaminergic neuronal cells are more vulnerable to Mn-induced ROS generation and apoptotic cell death than non-dopaminergic cells (Kitazawa et al., 2002), suggesting an increased sensitivity of dopaminergic neurons to metal neurotoxic insult. The literature suggests that metals in an ROS-rich environment may augment the oxidative insult by forming dopamine-derived highly cytotoxic radicals (Junn and Mouradian, 2001;

Kitazawa et al., 2001; Kanthasamy et al., 2002) which may contribute to the enhanced susceptibility of dopaminergic neurons to metal-induced neurotoxicity. Vanadium reportedly produces ROS such as hydroxyl free radicals (Cortizo et al., 2000; Gandara et al., 2005) and superoxide, which are further converted to $\mathrm{H}_{2} \mathrm{O}_{2}$ (Ding et al., 1994). Excessive ROS generation initiates the peroxidative decomposition of the phospholipids of cellular membranes, leading to the propagation of cellular injury. In the present study, we observed that exposure to vanadium increased the generation of $\mathrm{H}_{2} \mathrm{O}_{2}$ in $\mathrm{N} 27$ dopaminergic cells and that an antioxidant cocktail consisting of vitamin E, catalase, superoxide dismutase (SOD) and glutathione can completely prevent vanadium-induced neurotoxicity. Together, our results suggest that oxidative stress plays a major role in the neurotoxic effect of vanadium in dopaminergic neuronal cells. 
ROS has been shown to induce cytochrome $\mathrm{c}$ release from the mitochondria to cytosol through opening of the mitochondrial transition pore (Liu et al., 1996; Lee and Wei, 2000), and the released cytochrome $\mathrm{c}$ serves as a trigger for activation of the caspase-dependent apoptotic cell death cascade (Kaul et al., 2003; Kanthasamy et al., 2005). We observed an accumulation of cytochrome $\mathrm{c}$ in the cytosol of vanadium treated N27 cells within $3 \mathrm{~h}$ of metal exposure, suggesting that ROS generation, together with the release of cytochrome c from mitochondria may be early events in the vanadium-induced apoptotic cascade. We and others have shown that the initiator caspase- 9 and effector caspase- 3 play a critical role in the regulation and execution of apoptosis in dopaminergic neuronal cells (Anantharam et al., 2001; Kaul et al., 2003; Kanthasamy et al., 2003; Cassarino et al., 1999; Dodel et al., 1999). We observed that vanadium exposure dramatically increased both caspase- 9 and caspase- 3 in a time-dependent manner. Importantly, the caspase inhibitor Z-VAD-FMK significantly protected against vanadium-induced cell death, emphasizing the critical role of caspase activation in this cell death pathway. In terms of PD pathogenesis, caspase- 3 activation is a key contributor to apoptosis in dopaminergic neurons in human PD patients as well as in animal models of PD (Hartmann et al., 2000).

Our lab previously established that proteolytic activation of $\mathrm{PKC} \delta$ by caspase- 3 is an important event in the apoptotic cell death of dopaminergic cells following neurotoxic insults (Kanthasamy et al., 2003; Kanthasamy et al., 2005; Kaul et al., 2005). We showed that Mn treatment induces caspase-3-dependent proteolytic cleavage of $\mathrm{PKC} \delta$ but not of other isoforms, including $\mathrm{PKC} \alpha$ or $\mathrm{PKC} \beta$, suggesting that the cleavage is isoform-specific (Latchoumycandane et al., 2005). In this study, we showed that vanadium treatment resulted 
in $\mathrm{PKC} \delta$ proteolytic cleavage and kinase activation. Co-treatment with the caspase inhibitor Z-VAD-FMK significantly blocked vanadium-induced $\mathrm{PKC} \delta$ proteolytic cleavage as well as the kinase activity, demonstrating caspase-3 dependent $\mathrm{PKC} \delta$ activation during vanadium exposure in dopaminergic neuronal cells. The caspase inhibitor also blocked vanadiuminduced DNA fragmentation, indicating a role for a caspase-dependent apoptotic cascade in vanadium-induced apoptosis. Furthermore, the knockdown of PKC $\delta$ by siRNA completely suppressed vanadium-induced DNA fragmentation, suggesting that $\mathrm{PKC} \delta$ has a proapoptotic function in vanadium-induced dopaminergic neurotoxicity. The events downstream of PKC $\delta$ activation that lead to apoptotic cell death are still unclear. We recently showed that PKC $\delta$ translocates to the mitochondria and nucleus following proteolytic activation of the kinase (Sun et al., 2007; 2009). Mn has been reported to inhibit dopamine uptake in striatal synaptosomes (Chen et al., 2006) suggesting that Mn may affect the presynaptic dopaminergic neuronal terminal. We also further showed that vanadium inhibits in a dosedependent manner the uptake of dopamine in nigral primary neurons indicating that vanadium may also be neurotoxic to dopaminergic neurons in primary nigral cultures. Future studies will focus on identifying key downstream signaling molecules in vanadium-induced dopaminergic degeneration using both cell culture and animal models.

In conclusion, this study demonstrates for the first time that exposure to vanadium induces dopaminergic degeneration by a novel apoptotic pathway mediated by caspase-3dependent proteolytic activation of $\mathrm{PKC} \delta$ (Fig. 10). Our current study not only establishes caspase-mediated PKC $\delta$ cleavage as a key downstream event of vanadium-induced apoptosis but also emphasizes that blocking ROS, blocking caspase activity and selective targeting of 
the proapoptotic kinase PKC $\delta$ by siRNA can rescue dopaminergic neurons from vanadiuminduced dopaminergic degeneration. These findings support the hypothesis that environmental exposure to metals may play an important role in the etiopathogenesis of Parkinsons disease. To further strengthen these findings, a systematic analysis of the vanadium neurotoxic response in the nigrostriatal dopaminergic system in animal models of vanadium neurotoxicity is still warranted.

\section{$\underline{\text { Acknowledgement }}$}

This work was supported by National Institutes of Health (NIH) Grants ES10586 and NS 38644. The W. Eugene and Linda Lloyd Endowed Chair to AGK also is acknowledged. The ICP-MS device was provided by the U. S. Department of Energy, Nuclear Nonproliferation and Basic Energy Sciences Programs. Ames Laboratory is operated under Contract DE-AC02-07CH11358. The authors acknowledge Ms. MaryAnn deVries for her assistance in the preparation of this manuscript. 


\section{$\underline{\text { References }}$}

Alessio, L., and Lucchini, R., 1996. In: Data profiles for selected chemicl series. Argentesi F., Roi R., Sevilla Marcos JM. (eds.) (European Commission Joint Research Project Centre, Luxembourg. SPI 96, 59.)

Amorim, F.A.C., Welz, B., Costa, A.C.S., Lepri, F.G., Vale, M.Goreti.R., Ferreira, S.L.C., 2007 Determination of vanadium in petroleum and petroleum products using atomic spectrometric techniques Talanta, 72 , 349-359.

Anantharam, V., Kitazawa, M., Wagner, J., Kaul, S., and Kanthasamy, A. G., 2002. Caspase3-dependent proteolytic cleavage of protein kinase Cdelta is essential for oxidative stressmediated dopaminergic cell death after exposure to methylcyclopentadienyl manganese tricarbonyl. J Neurosci. 22, 1738-51.

Anantharam, V., Kitazawa M., Latchoumycandane, C., Kanthasamy, A. and Kanthasamy, A.G., 2004. Blockade of PKCdelta proteolytic activation by loss of function mutants rescues mesencephalic dopaminergic neurons from methylcyclopentadienyl manganese tricarbonyl (MMT)-induced apoptotic cell death, Ann NY Acad Sci. 1035, 271-289.

Andersen, J.K., 2004. Oxidative stress in neurodegeneration: cause or consequence. Nature Rev. S18-23.

Anglade, P., Vyas, S., Javoy-Agid, F., Herrero, M.T., Michel, P.P., Marquez, J., MouattPrigent, A., Ruberg, M., Hirsch, E.C., Agid Y., 1997. Apoptosis and autophagy in nigral neurons of patients with Parkinson's disease. Histol Histopathol. 12, 25-31.

Aschner, M., and Aschner, J. ,1991. Manganese neurotoxicity: Cellular effects and bloodbrain barrier transport. Neurosci. Biobehav. Rev. 15, 333-340.

Avila-Costa, M.R., Montiel, Flores. E., Colín-Barenque, L., Ordoñez, J.L., Gutiérrez, A.L., Niño-Cabrera, G., Mussali-Galante, P., Fortoul, T.I. 2004. Nigrostriatal modifi cations after vanadium inhalation: An immunocytochemical and cytological approach. Neurochem Res. $29,1365-9$.

Avila-Costa, M.R., Fortoul, T.I., Niño-Cabrera, G., Colín-Barenque, L., Bizarro- Nevares, P., Gutiérrez-Valdez, A.L., Ordóñez-Librado, J.L., Rodríguez-Lara, V., Mussali-Galante, P., Díaz-Bech, P., Anaya-Martínez, V., 2006. Hippocampal cell alterations induced by the inhalation of vanadium pentoxide promote memory deterioration. Neurotoxicology. 27,100712.

Avila-Costa, M.R., Colín-Barenque, L., Zepeda-Rodríquez, A., Antuna, S.B., Saldivar, O.L., Espejel-Maya, G., Mussali-Galante, P., Avila-Casado, M.C., Reyes-Olivera, A., AnayaMartinez, V., and Fortoul, T.I., 2005. Ependymal epithelium disruption after vanadium pentoxide inhalation. A mice experimental model. Neurosci Lett. 381, 21-5. 
Barceloux, D.G., 1999. Vanadium. J. Toxicol. Clin. Toxicol. 37, 265-278.

Brodie, C. and Blumberg, P.M., 2003. Regulation of cell apoptosis by protein kinase c delta. Apoptosis. 8, 19-27.

Brown, D.R., Herms, J., and Kretzschmar, H.A., 1994. Mouse cortical cells lacking cellular PrP survive in culture with a neurotoxic PrP fragment. Neuroreport. 5, 2057-60.

Bunting Robert M. Vanadium: How market developments affect the titanium industry. Strategic minerals corporation. Titanium 2006, International Titanium Association Conference, San Diego, California, October 3, 2006.

Cantuti-Castelvetri, I., Shukitt-Hale, B., and Joseph, J.A., 2003. Dopamine neurotoxicity: age-dependent behavioral and histological effects. Neurobiol Aging. 24, 697-706.

Cassarino, D.S., Parks, J.K., Parker Jr, W.D., Bennett Jr, J.P., 1999. The parkinsonian neurotoxin MPP+ opens the mitochondrial permeability transition pore and releases cytochrome $\mathrm{c}$ in isolated mitochondria via an oxidative mechanism. Biochim Biophys Acta. $1453,49-62$.

Castello, P.R., Drechsel, D.A., and Patel, M., 2007. Mitochondria are a major source of paraquat-induced reactive oxygen species production in the brain. J Biol Chem. 282, 1418614193.

Chen, M.K., Lee, J.S., McGlothan, J.L., Furukawa, E., Adams, R.J., Alexander, M., Wong, D.F., Guilarte, T.R., 200. Acute manganese administration alters dopamine transporter levels in the non-human primate striatum. Neurotoxicology. 27, 229-236.

Choi, C.J., Anantharam, V., Saetveit, N.J., Houk, R.S., Kanthasamy, A., Kanthasamy, A.G., 2007. Normal cellular prion protein protects against manganese-induced oxidative stress and apoptotic cell death. Toxicol Sci. 98, 495-509.

Chun, H.S., Lee, H., and Son, J.H., 2001. Manganese induces endoplasmic reticulum (ER) stress and activates multiple caspases in nigral dopaminergic neuronal cells, SN4741. Neurosci Lett. 316, 5-8.

Cranmer, J., Mergler, D., Williams-Johnson, M., 1999. (eds.) Manganese. Are there effects from long-term, low-level exposure ? Neurotoxicology. 20, 2-3.

Crossgrove, J.S., and Zheng, W., 2004. Manganese toxicity upon overexposure. NMR in Biomedicine. 17, 544-553. 
Cortizo, A.M., Bruzzone, L., Molinuevo, S., Etcheverry, S.B., 2000. A possible role of oxidative stress in the vanadium-induced cytotoxicity in the MC3T3E1 osteoblast and UMR106 osteosarcoma cell lines. Toxicology.147, 89-99.

Dauer, W. and Przedborski, S., 2003. Parkinson's disease: mechanisms and models. Neuron. 39, 889-909.

Dawson, V. L. and Dawson, T. M., 1996. Nitric oxide neurotoxicity. J. Chem. Neuroanat. 10, 179-190.

Dawson, T.M., and Dawson, V.L., 2003. Molecular pathways of neurodegeneration in Parkinson's disease. Science (Wash DC). 302, 819-822.

Ding, M., Gannett, P.M., Rojanasakul, Y., Liu K.J. and Shi X.L., 1994. One-electron reduction of vanadate by ascorbate and related free-radical generation at physiological $\mathrm{pH}, \mathrm{J}$. Inorg. Biochem. 55, 101-112.

Dodel, R.C., Du, Y., Bales, K.R., Ling, Z., Carvey, P.M., Paul, S.M., 1999. Caspase-3-like proteases and 6-hydroxydopamine induced neuronal cell death. Brain Res Mol Brain Res. 64, 141-148.

Done, A.K., 1979. Of metals and chelation. Emer Med, 11, 186-218.

Donze, O., Picard, D., 2002. RNA interference in mammalian cells using siRNAs synthesized with T7 RNA polymerase. Nucleic Acids Res. 30:e46.

Elbashir, S.M., Lendeckel, W., Tuschl, T., 2001. RNA interference is mediated by 21- and 22-nucleotide RNAs. Genes Dev. 15, 188-200.

Ellingsen, D.G., Konstantinov, R., Bast-Pettersen, R., Merkurjeva, L., Chashchin, M., Thomassen Y., Chashchin, V., 2008 A neurobehavioral study of current and former welders exposed to manganese. Neurotoxicology. 29, 48-59.

Erikson, K. M., Syversen, T., Steinnes, E., and Aschner, M., 2004. Globus pallidus: A target brain region for divalent metal accumulation associated with dietary iron deficiency. J. Nutr. Biochem. 15, 335-341.

Fleming, L., Mann, J.B., Bean, J., Briggle, T., Sanchez-Ramos, J.R., 1994. Parkinson's disease and brain levels of organochlorine pesticides. Ann Neurol. 36,100-103.

Gandara, R.M.C., Soares, S.S, Martins, H., Gutierrez-Merino, C., Aureliano, M., 2005. Vanadate oligomers: In vivo effects in hepatic vanadium accumulation and stress markers. $\mathrm{J}$ Inorg Biochem. 99, 1238-44. 
Ghayur, T., Hugunin, M., Talanian, R. V., Ratnofsky, S., Quinlan, C., Emoto, Y., Pandey, P., Datta, R., Huang, Y., Kharbanda, S., Allen, H., Kamen, R., Wong, W., and Kufe, D,. 1996. Proteolytic activation of protein kinase $\mathrm{C} \delta$ by an ICE/CED 3-like protease induces characteristics of apoptosis. J. Exp. Med. 184, 2399-2404.

Goldman, S.M., Tanner, C.M., Olanow, C. W., Watts, R. L., Field, R.D., Langston, J.W., 2005. Occupation and parkinsonism in three movement disorders clinics. Neurology. 65, $1430-1435$

Gorell, J.M., Johnson, C.C., Rybicki, B.A., Peterson, E.L., Kortsha, G.X., Brown, G.G., Richardson, R.J., 1997. Occupational exposures to metals as risk factors for Parkinson's disease, Neurology. 48, 650-658.

Hamai, D., and Bondy, S. C., 2004. Pro- or anti-oxidant manganese: a suggested mechanism for reconciliation. Neurochem. Int. 44, 223-229.

Hartmann, A., Hunot, S., Michel, P.P., Muriel, M.P., Vyas, S., Faucheux, B.A., MouattPrigent, A., Turmel, H., Srinivasan, A., Ruberg, M., Evan, G.I., Agid, Y., Hirsch, E.C., 2000. Caspase-3: a vulnerability factor and final effector in apoptotic death of dopaminergic neurons in Parkinson's disease. Proc Natl Acad Sci USA. 97, 2875-2880.

Hazardous Substance Database, ChemIDPlus., 2006.

Hirata, Y., 2002. Manganese-induced apoptosis in PC12 cells. Neurotoxicol Teratol. 24, 639

Junn, E,. and Mouradian, M.M., 2001. Apoptotic signaling in dopamine-induced cell death: the role of oxidative stress, p38 mitogen-activated protein kinase, cytochrome c and caspases. J Neurochem. 78, 374-383.

Kanthasamy, A.G., Sharma, N., Kirby, M.L., Schwarzschild, M.A., 2002. Neuroprotective strategies in Parkinson's disease. In: Neuroprotection: Basic \& Clinical Aspects. Prominent Press, pp. 604-640.

Kanthasamy, A. G., Kitazawa, M., Kanthasamy, A., and Anantharam, V., 2003. Role of proteolytic activation of protein kinase $\mathrm{C}$ delta $(\mathrm{PKC} \delta$ ) in oxidative stress-induced apoptosis. Antioxid. Redox Signal. 5, 609-620

Kanthasamy, AG., Kitazawa, M., Kanthasamy, A and Anantharam, V., 2005. DieldrinInduced Neurotoxicity: Relevance to Parkinson's Disease Pathogenesis. Neurotoxicology. 26, 701-19.

Kaul, S., Kanthasamy, A., Kitazawa, M., Anantharam, V., and Kanthasamy, A. G., 2003. Caspase-3 dependent proteolytic activation of protein kinase $\mathrm{C}$ delta mediates and regulates 1-methyl-4-phenylpyridinium (MPP+)-induced apoptotic cell death in dopaminergic cells: relevance to oxidative stress in dopaminergic degeneration. Eur J Neurosci. 18, 1387-401. 
Kaul, S., Anantharam, V., Kanthasamy, A., Kanthasamy, AG., 2005(a). Wild-type alphasynuclein interacts with pro-apoptotic proteins PKCdelta and BAD to protect dopaminergic neuronal cells against MPP+-induced apoptotic cell death. Brain Res Mol Brain Res.139, $137-152$.

Kaul, S., Anantharam, V., Yang, Y., Choi, C. J., Kanthasamy, A., Kanthasamy, A. G. 2005(b). Tyrosine phosphorylation regulates the proteolytic activation of protein kinase Cdelta in dopaminergic neuronal cells. J Biol Chem. 280, 28721-30.

Kikkawa, U., Matsuzaki H, and Yamamoto T., 2002. Protein kinase Cdelta (PKC-delta): activation mechanisms and functions. J Biochem. 132, 831-839.

Kitazawa, M., Anantharam, V., and Kanthasamy, A. G., 2001. Dieldrin-induced oxidative stress and neurochemical changes contribute to apoptopic cell death in dopaminergic cells. Free Radic Biol Med. 31, 1473-85.

Kitazawa, M., Wagner, J. R., Kirby, M. L., Anantharam, V., and Kanthasamy, A. G., 2002. Oxidative stress and mitochondrial-mediated apoptosis in dopaminergic cells exposed to methylcyclopentadienyl manganese tricarbonyl. J Pharmacol Exp Ther. 302, 26-35.

Kitazawa, M., Anantharam, V., and Kanthasamy, A.G., 2003. Dieldrin induces apoptosis by promoting caspase-3-dependent proteolytic cleavage of protein kinase Cdelta in dopaminergic cells: relevance to oxidative stress and dopaminergic degeneration. Neuroscience. 119, 945-64.

Latchoumycandane, C., Anantharam, V., Kitazawa, M., Yang, Y., Kanthasamy, A., Kanthasamy, A.G., 2005. Protein kinase $\mathrm{C} \delta$ is a key downstream mediator of manganeseinduced apoptosis in dopaminergic neuronal cells. J Pharmacol Exp Ther. 313, 46-55.

Lee, H.C., Wei, Y.H., 2000. Mitochondrial role in life and death of the cell. J Biomed Sci. 7, $2-15$.

Leist, M., Volbracht, C., Fava, E., Nicotera, P., 1998. 1-Methyl-4-phenylpyridinium induces autocrine excitotoxicity, protease activation, and neuronal apoptosis. Mol Pharmacol. 54, 789-801.

Li, L., Lorenzo, P.S., Bogi, K., Blumberg, P.M., Yuspa, S.H., 1999. Protein kinase Cdelta targets mitochondria, alters mitochondrial membrane potential, and induces apoptosis in normal and neoplastic keratinocytes when overexpressed by an adenoviral vector. Mol Cell Biol. 19, 8547-8558.

Liou, H.H., Tsai, M.C., Chen, C.J., Jeng, J.S., Chang, Y.C., Chen, S.Y., Chen, R.C., 1997. Environmental risk factors and Parkinson's disease: a case-control study in Taiwan. Neurology. 48,1583-1588. 
Lucchini, R., Selis, L., Folli, D., Apostoli, P., Mutti, A., Vanoni, O., Iregren, A., Alessio, L., 1995. Neurobeha- vioral effects of manganese in workers from a ferroalloy plant after temporary cessation of exposure. Scand. J. Work. Environ. Health. 21, 143-149.

Lucchini, R., Bergamaschi, E., Smargiassi, A., Apostoli, P. 1997. Motor function, olfactory threshold and haematological indices in manganese exposed ferroalloy workers. Environmental Research 73, 175-180.

Marder, K., Logroscino, G., Alfaro, B., Mejia, H., Halim, A., Louis, E., Cote, L., Mayeux, R., 1998. Environmental risk factors for Parkinson's disease in an urban multiethnic community. Neurology. 50, 279-281.

Majumder, P.K., Mishra, N.C., Sun, X., Bharti, A., Kharbanda, S., Saxena, S. and Kufe, D., 2001. Targeting of protein kinase $\mathrm{C} \delta$ to mitochondria in the oxidative stress response. Cell

Growth Differ. 129, 465-470.

Mastore, M., Kohler, L. and Nappi, A.J., 2005. Production and utilization of hydrogen peroxide associated with melanogenesis and tyrosinase-mediated oxidations of DOPA and dopamine. FEBS J. 272, 2407-2415.

McNeilly, J.D., Heal, M.R., Beverland, I.J., Howe, A., Gibson, M.D., Hibbs, L.R., MacNee W., Donaldson, K., 2004. Soluble transition metals cause the pro-inflammatory effects of welding fumes in vitro. Toxicol Appl Pharmacol. 196, 95-107.

Mergler, D., 1999. Neurotoxic effects of low level exposure to manganese in human populations. Environ Res. 80, 99-102.

Michel, P. P., Hefti, F., 1990 Toxicity of 6-hydroxydopamine and dopamine for dopaminergic neurons in culture. J Neurosci Res. 26, 428-435.

Olanow, C.W., 2004. Manganese-Induced parkinsonism and Parkinson's Disease. Ann. N.Y. Acad. Sci. 1012, 1-15.

Olanow, C.W., Good, P.F., Shinotoh, H., Hewitt, K.A., Vingerhoets, F., Snow, B.J., Beal, M.F., Calne, D.B., Perl, D.P., 1996. Manganese intoxication in the rhesus monkey: a clinical, imaging, pathologic, and biochemical study. Neurology, 46, 492-8.

Olanow, C.W., Tatton, W.G., 1999. Etiology and pathogenesis of Parkinson's disease. Annu Rev Neurosci. 22, 123-44.

Park, R.M., Schulte, P.A., Bowman, J.D., Walker, J.T., Bondy, S.C., Yost, M.G., Touchstone, J.A., Dosemeci, M., 2005. Potential occupational risks for neurodegenerative diseases. Am J Ind Med. 48, 63-77. 
Priyadarshi, A., Khuder, S.A., Schaub, E.A., Shrivastava, S., 2000. A meta-analysis of Parkinson's disease and exposure to pesticides. Neurotoxicology. 21, 435-440.

Pyrzynska, K., Weirzbicki, T., 2004. Determination of vanadium species in environmental samples. Talanta. 64, 823-829.

Racette, B.A., McGee-Minnich, L., Moerlein, S.M., Mink, J.W., Videen, T.O., Perlmutter, J.S., 2001. Welding-related parkinsonism: clinical features, treatment, and pathophysiology. Neurology. 56, 8-13.

Ritz, B., Yu, F., 2000. Parkinson's disease mortality and pesticide exposure in California 1984- 1994. Int J Epidemiol. 29, 323-329.

Sabbioni, E., Marafante, E., Rade, J., Gregotti, C., Di Nucci, A., Manzo, L., 1981. Biliary excretion of vanadium in rats. Toxicol. Eur. Res. 3, 93-98.

Sabbioni, E., Pozzi, G., Pintar, A., Cassella, L., Garattini, S., 1991. Cellular retention cytotoxicity and morphological transformation by vanadium(IV) and vanadium(V) in BALB/3T3 cell lines. Carcinogenesis. 12, 47-52.

Schwarz, A., Burwinkel, M., Riemer, C., Schultz, J., Baier, M., 2004. Unchanged Scrapie Pathology in Brain Tissue of Tyrosine Kinase Fyn-Deficient Mice. Neurodegenerative Dis. 1, 266-268.

Schrantz, N., Blanchard, D.A., Mitenne, F., Auffredou, M.T., Vazquez, A., and Leca, G. 1999 Manganese induces apoptosis of human B cells: caspase-dependent cell death blocked by bcl-2. Cell Death Differ. 6, 445-453.

Schulte, P. A., Burnett, C. A., Boeniger, M. F., Johnson, J., 1996. Neurodegenerative diseases: occupational occurrence and potential risk factors, 1982 through 1991. Am J Public Health. 86,1281-1288.

Shum, S. C., Neddersen, R., and Houk, R. S., 1992. Elemental speciation by liquid chromatography-inductively coupled plasma mass spectrometry with direct injection nebulization. Analyst. 117, 577-82.

Smargiassi, A., Mutti, A., De Rosa, A., De Palma, G., Negrotti, A., Calzetti, S. 1998. A casecontrol study of occupational and environmental risk factors for Parkinson's disease in the Emilia-Romagna region of Italy. Neurotoxicology. 19, 709-712.

Soazo, M., Garcia, G.B., 2007. "Vanadium exposure through lactation produces behavioral alterations and CNS myelin deficit in neonatal rats". Neurotoxicology and Teratology. 29, 503-510. 
Sun, F., Kanthasamy A., Anantharam, V., Kanthasamy, A.G., 2007. Environmental neurotoxic chemicals-induced ubiquitin proteasome system dysfunction in the pathogenesis and progression of Parkinson's disease. Pharmacology and Therapeutics, 114, 327-344.

Sun, F., Kanthasamy, A., Anantharam, V., Kanthasamy, A.G., 2009. Mitochondrial accumulation of polyubiquitinated proteins and differential regulation of apoptosis by polyubiquitination sites Lys-48 and -63. J Cell Mol Med. 2009 - published online

Taylor, C. A., Saint-Hilaire, M. H., Cupples, L. A., Thomas, C. A.; Burchard, A. E., Feldman, R. G., Myers, R. H. 1999. Environmental, medical, and family history risk factors for Parkinson's disease: a New England-based case control study. Am J Med Genet. 88, 742749.

Tuchsen, F., Jensen, A. A., 2000., Agricultural work and the risk of Parkinson's disease in Denmark, 1981- 1993. Scand J Work Environ Health. 26, 359-362.

Vega, I.E., Cui, L., Propst, J.A., Hutton, M.L., Lee, G., Yen, S.H., 2005. Increase in tau tyrosine phosphorylation correlates with the formation of tau aggregates. Brain Res Mol Brain Res. 138, 135-44.

Vaglini, F., Pardini, C., Viaggi, C., Caramelli, A., Corsini, G. U., 2008. Apomorphine offers new insight into dopaminergic neuron vulnerability in mesencephalic cultures. Neuropharmacology. 55, 737-742.

WHO. Vanadium. Chapter 6.12. WHO Regional Offi ce for Europe, Copenhagen: Denmark; 2000. p. 9.

Wu, J., Riyaz Basha, Md., Brock, B., Cox, David P., Cardozo-Pelaez, F., McPherson, C.A., Harry, J., Rice, D.C., Maloney, B., Chen, D., Lahiri, D.K. and Zawia, N.H., 2008. Alteration of Alzheimer disease (AD)-like pathology in aged monkeys after infantile exposure to environmental lead $(\mathrm{Pb})$ : Evidence for a developmental origin and environmental link for AD. J. Neurosci. 28, 3-9.

Yang, Y., Kaul, S., Zhang, D., Anantharam, V., Kanthasamy, A.G., 2004. Suppression of caspase-3-dependent proteolytic activation of protein kinase $\mathrm{C}$ delta by small interfering RNA prevents MPP+-induced dopaminergic degeneration. Mol Cell Neurosci. 25, 406-421.

Zhang, D., Anantharam, V., Kanthasamy, A., and Kanthasamy, A.G., 2007. Neuroprotective Effect of Protein Kinase C $\delta$ Inhibitor Rottlerin in Cell Culture and Animal Models of Parkinson's Disease J Pharmacol Exp Ther. 322, 913-922. 


\section{Figures and Figure Legends}

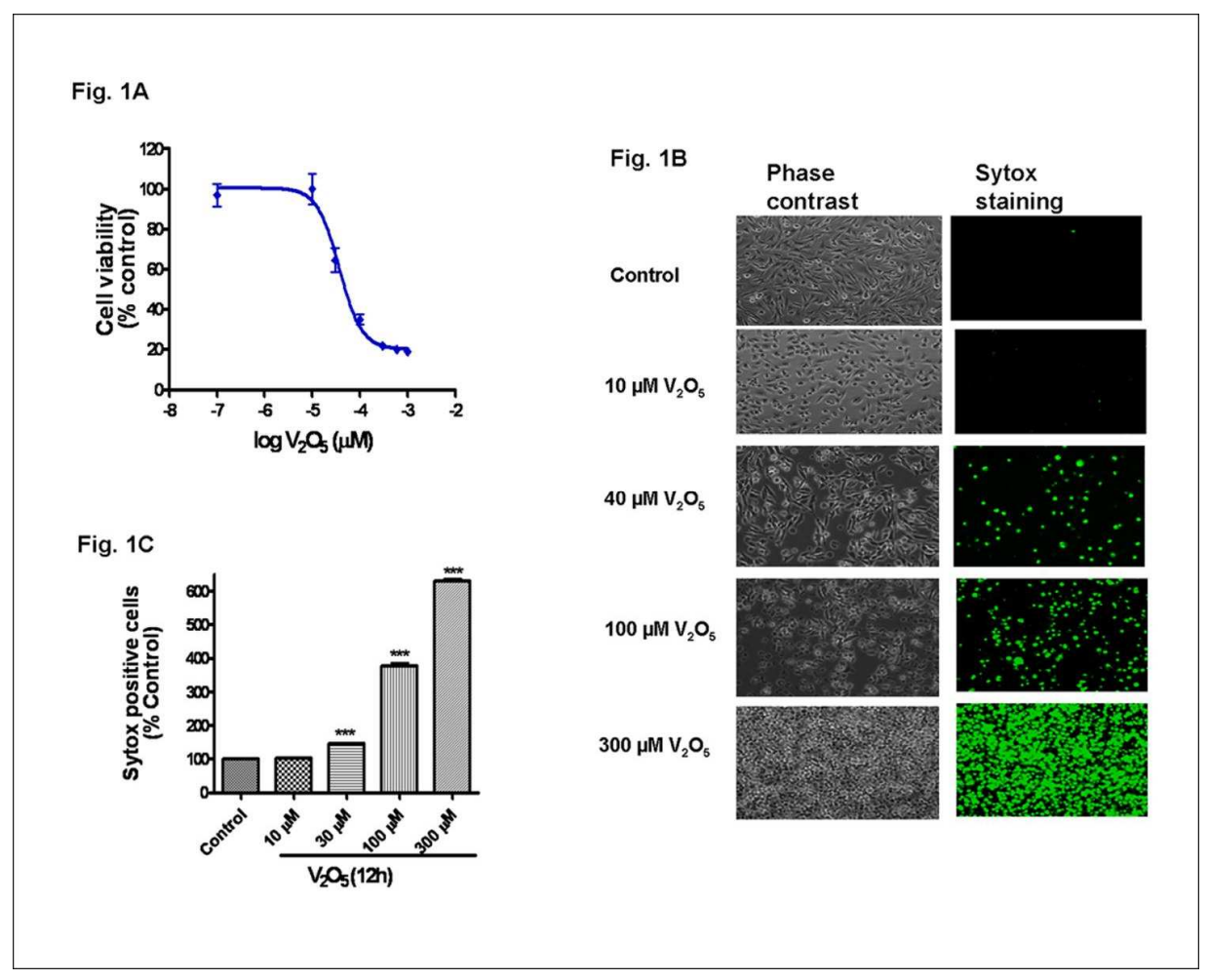

Fig. 1 Vanadium induces a dose-dependent neurotoxic effect on N27 dopaminergic neuronal cells. (A) Effect of vanadium on cell viability in N27 dopaminergic neuronal cells. The cells were exposed to $0-300 \mu \mathrm{M} \mathrm{V}_{2} \mathrm{O}_{5}$ and then cell viability was measured using the MTT assay. The value of vanadium neurotoxicity in $\mathrm{N} 27$ dopaminergic cells was $\mathrm{EC}_{50}=37 \pm 3.47 \mu \mathrm{M}$. Data represent results from at least eight individual measurements. (B) Visualization of vanadium-induced neurotoxicity by Sytox green fluorescence assay. N27 dopaminergic neuronal cells were exposed to $40 \mu \mathrm{M} \mathrm{V}_{2} \mathrm{O}_{5}$ for $12 \mathrm{~h}$ and then cells were loaded with Sytox green and observed under a Nikon inverted fluorescence microscope and pictures were captured with a SPOT digital camera (Diagnostic Instruments, Sterling Heights, MI). (C) Quantitative analysis of vanadium-induced neurotoxicity was measured by the Sytox green cytotoxicity fluorescence assay. The Sytox fluorescence was measured by Bio-Tek fluorescence microplate reader. Data represent results from at least eight individual measurements and are expressed as mean \pm S.E.M. The values are expressed as a percentage of untreated control cells. ${ }^{* *} \mathrm{p}<0.001$ indicates significant difference with each of the other groups. 
Fig. 2A

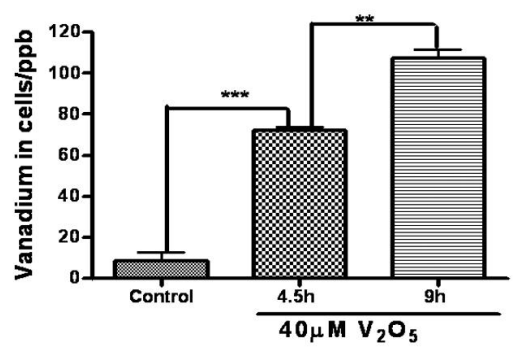

Fig. 2B

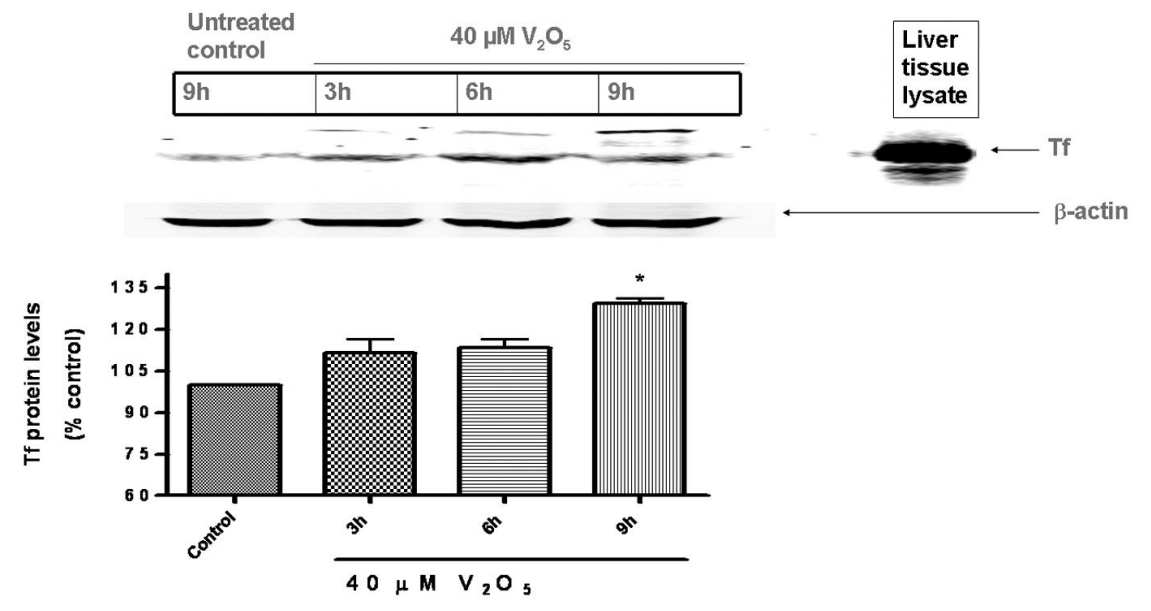

Fig. 2C

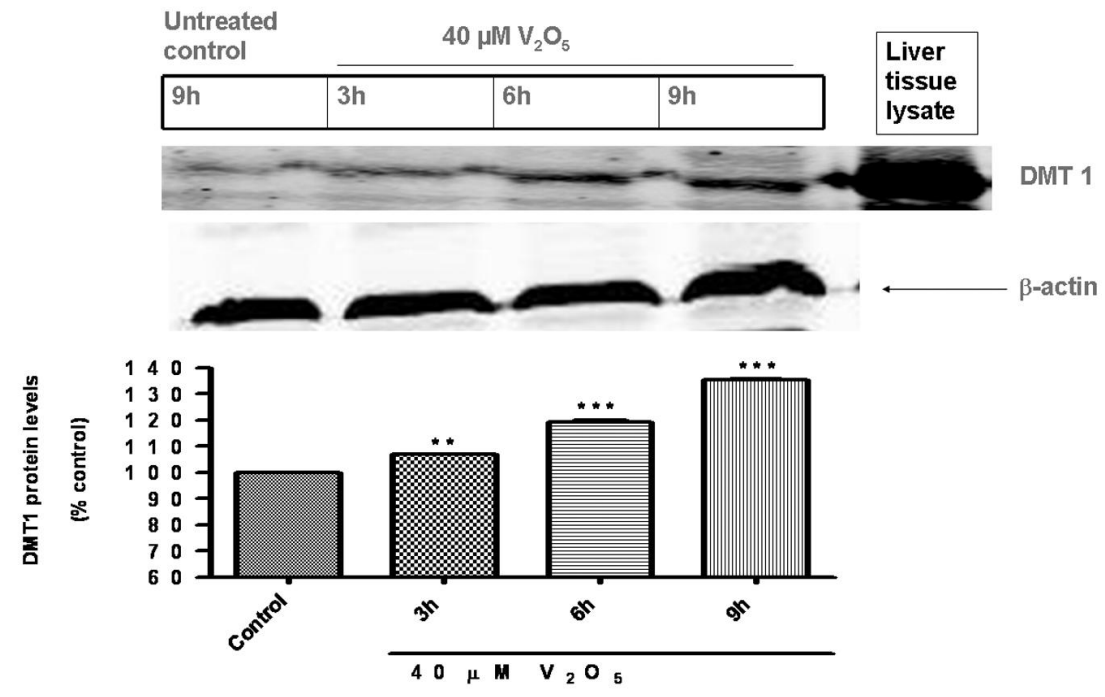


Fig. 2 Intracellular vanadium uptake and upregulation of metal transport proteins following vanadium exposure in N27 dopaminergic neuronal cells. (A) Vanadium uptake in N27 dopaminergic neuronal cells. N27 dopaminergic neuronal cells were exposed to $40 \mu \mathrm{M}$ vanadium for 4.5 and $9 \mathrm{~h}$ and the vanadium content of the cells was measured using the ICPMS technique. Data represent results from three individual measurements and are expressed as mean \pm S.E.M. $* * p<0.01$ and $* * * p<0.001$. Upregulation of Tf (B) and DMT1 (C). N27 cells were treated with $40 \mu \mathrm{M}$ vanadium for 3,6 , and $9 \mathrm{~h}$ and then DMT and Tf immunoblot was performed as described in the Methods. To confirm equal protein loading in each lane, the membranes were reprobed with B-actin antibody. Mouse liver lysates were used as a positive control. Data represent results from at least two separate experiments. Mouse liver lysates were used as a positive control. Data represent results from at least two separate measurements. Data represent results from at least two individual measurements and are expressed as mean \pm S.E.M. $* \mathrm{p}<0.05$, $* * \mathrm{p}<0.01$ and $* * * \mathrm{p}<0.001$ indicates significant difference with control. Vanadium uptake alters intracellular manganese $(\mathrm{Mn})$ concentration (D) and intracellular copper $(\mathrm{Cu})$ concentration $(\mathbf{E})$ in N27 dopaminergic neuronal cells. N27 dopaminergic neuronal cells were exposed to $40 \mu \mathrm{M}$ vanadium for 4.5 and $9 \mathrm{~h}$ and the intracellular $\mathrm{Mn}$ and $\mathrm{Cu}$ content of the cells was measured using the ICP-MS technique. Data represent results from three individual measurements and are expressed as mean \pm S.E.M. $* \mathrm{p}<0.05, * * \mathrm{p}<0.01$ and $* * * \mathrm{p}<0.001$ 
Fig. 3A

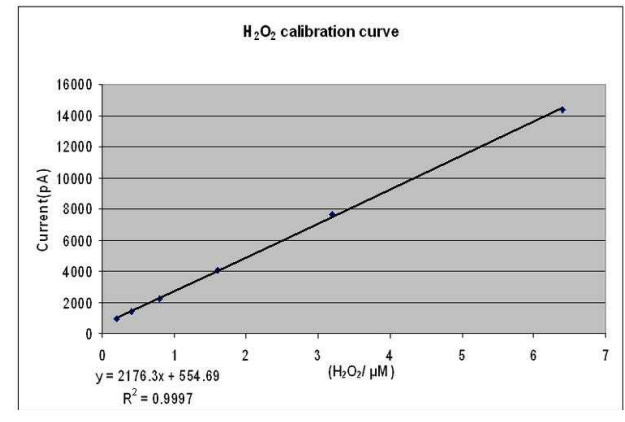

Fig. 3B

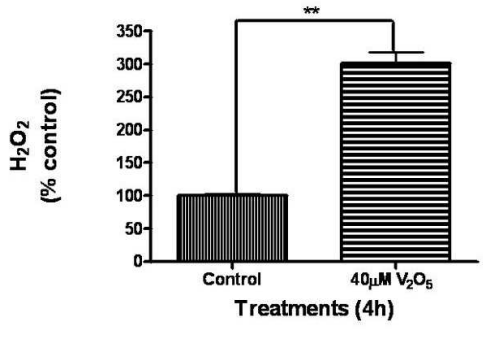

Fig. $3 \mathrm{C}$

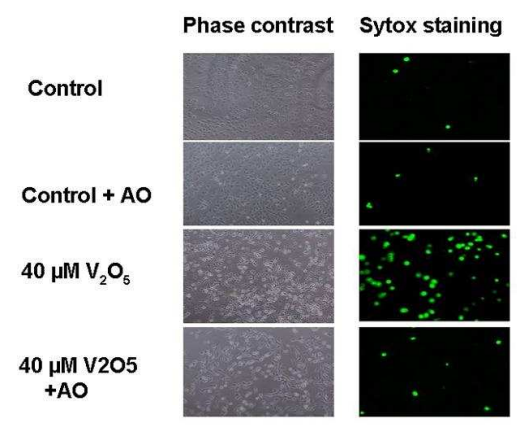

Fig. 3D

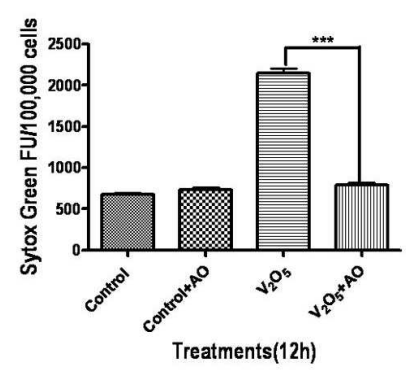

Fig. 3 Role of oxidative stress in vanadium-induced neurotoxicity in $\mathrm{N} 27$ dopaminergic neuronal cells. (A) Calibration curve of $\mathrm{H}_{2} \mathrm{O}_{2}$ production by polarography: $\mathrm{H}_{2} \mathrm{O}_{2}$ production was measured using an Apollo 4000 Free Radical Analyzer (WPI, Sarasota, FL) equipped with a $100-\mu \mathrm{m} \mathrm{H}_{2} \mathrm{O}_{2}$ sensor. The $100-\mu \mathrm{m} \mathrm{H}_{2} \mathrm{O}_{2}$ sensor probe was calibrated using various doses of $\mathrm{H}_{2} \mathrm{O}_{2}$ according to the manufacturer's instructions. The calibration curve was used to calculate the $\mathrm{H}_{2} \mathrm{O}_{2}$ in the control and treatment groups. (B) Vanadium induced $\mathrm{H}_{2} \mathrm{O}_{2}$ generation in N27 cells. The measurements were conducted in a 96 well plate containing N27 cells exposed to $40 \mu \mathrm{M}$ vanadium for $4 \mathrm{~h}$. Each measurement was started by insertion of the $100-\mu \mathrm{m} \mathrm{H}_{2} \mathrm{O}_{2}$ sensor probe into wells containing cells. The output signal was recorded and compared to the standard curve. (C) Effect of antioxidants on vanadium-induced neurotoxicity. N27 dopaminergic neuronal cells were co-treated with vanadium and an antioxidant solution cocktail (AO) of vitamin E, glutathione, superoxide dismutase, and catalase. The Sytox green was added to cells and then cells were observed under a Nikon inverted fluorescence microscope. The pictures were captured with a SPOT digital camera (Diagnostic Instruments, Sterling Heights, MI). (D) Quantitative analysis of the protective effect of $\mathrm{AO}$ on vanadium-induced neurotoxicity was measured by the Sytox green cytotoxicity fluorescence assay. Data represent results from four individual measurements and are expressed as mean \pm S.E.M. $* * p<0.001$ and $* * * p<0.01$. 
Fig. 4

\section{Cytosolic fraction}

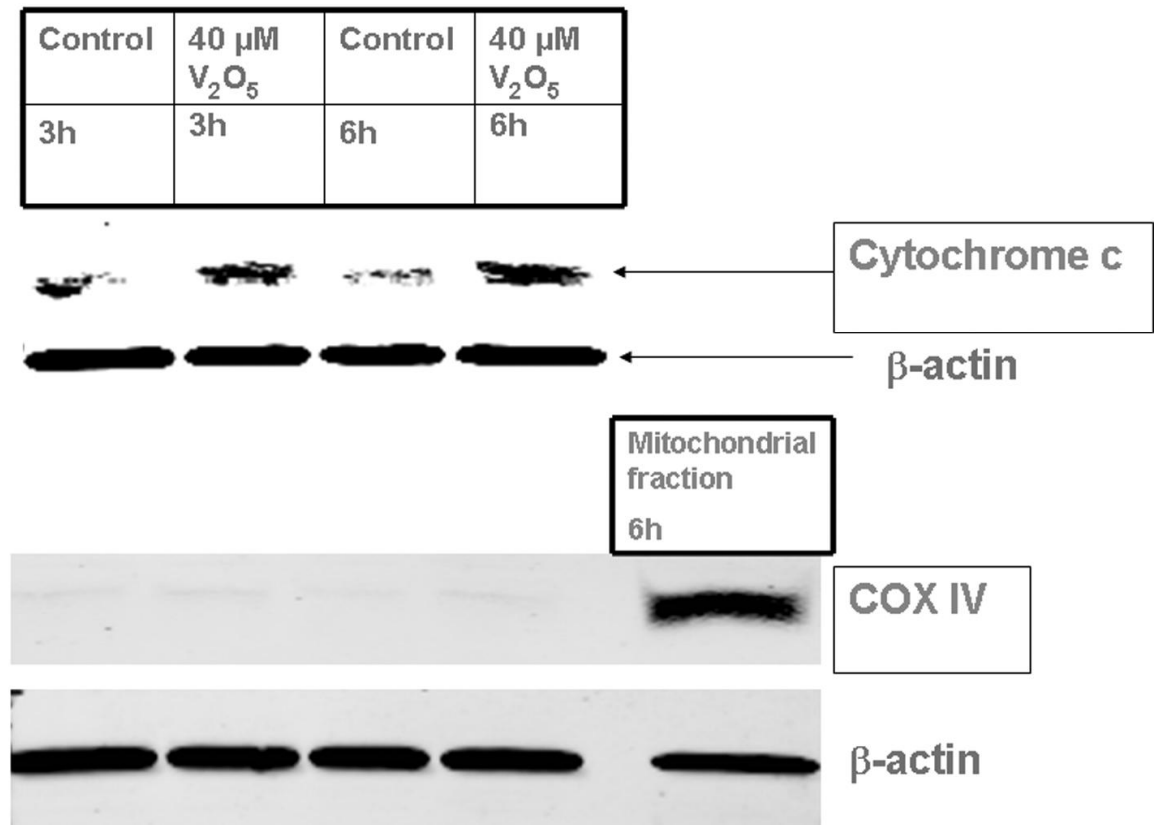

Fig. 4 Mitochondrial release of cytochrome $c$ in vanadium-treated N27 dopaminergic neuronal cells. The cells were treated with $40 \mu \mathrm{M}$ vanadium for 3 and $6 \mathrm{~h}$, cytosolic fractions were isolated, and cytochrome c was measured by Western blot. To confirm equal protein loading in each lane, the membranes were reprobed with $\beta$-actin antibody. The membranes were reprobed with COX-IV antibody to ensure purity of the cytosolic fraction and the mitochondrial fraction was used as a positive control. Data represent results from at least two separate measurements. 
Fig. 5A

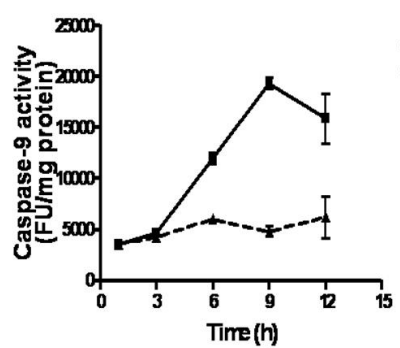

Fig. 5C

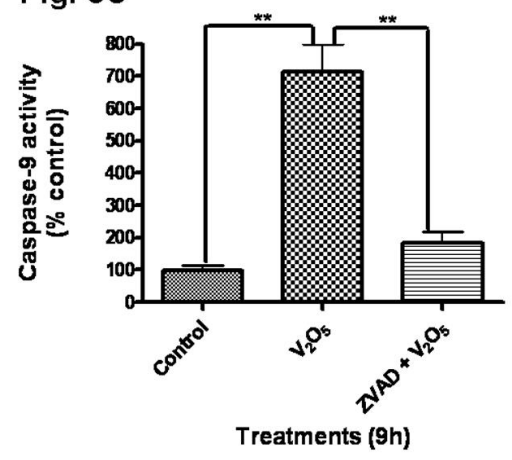

Fig. 5B

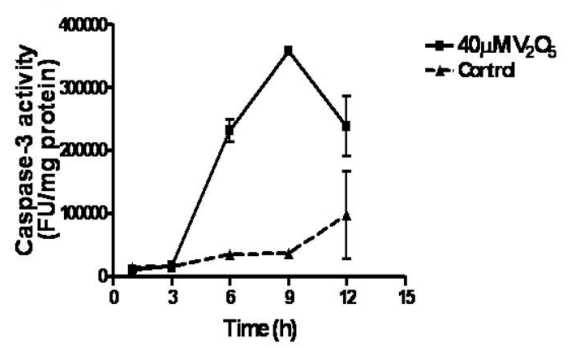

Fig. 5D

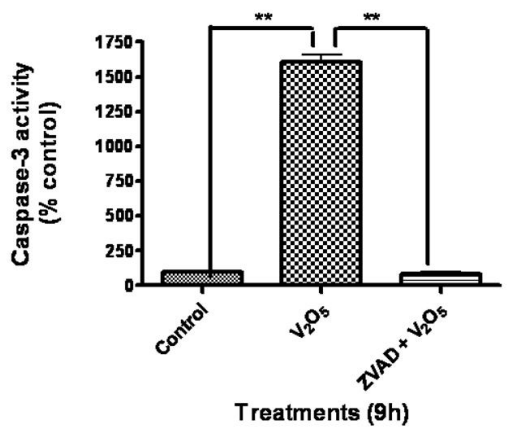

Fig. 5 Activation of caspases-9 and -3 in vanadium-treated N27 cells. N27 dopaminergic cells were treated with $40 \mu \mathrm{M}$ vanadium for 3, 6, 9 and $12 \mathrm{~h}$. Caspase-9 (A) and caspase-3 (B) enzyme activities were assayed using caspase-9 and caspase-3 substrates, respectively. Effect of Z-VAD-FMK on caspase-9 (C) and caspase-3 (D) was tested following cotreatment with vanadium and the caspase inhibitor for $9 \mathrm{~h}$. Data represent results from at least six individual measurements and are expressed as mean \pm S.E.M. ${ }^{*} \mathrm{p}<0.01$. 
Fig. 6A

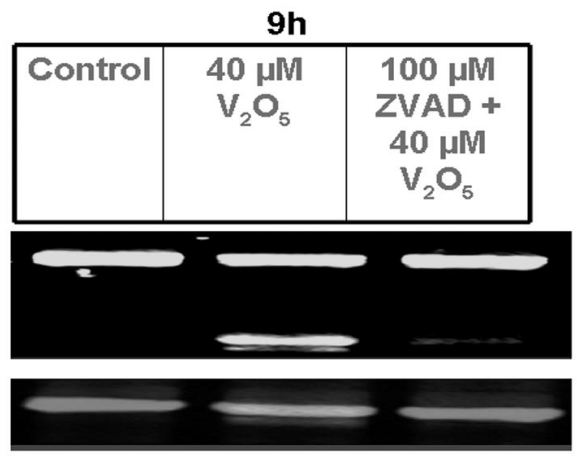

Native PKC $\delta$

Cleaved PKC $\delta$

$\beta$-actin

Fig. 6B

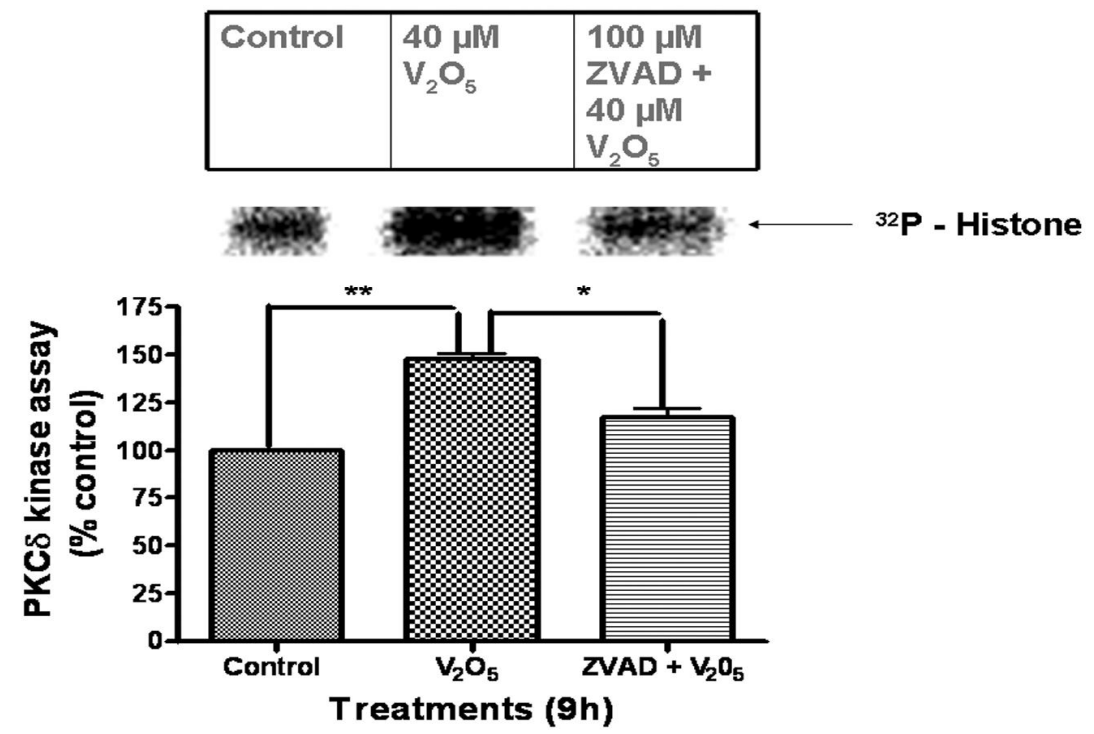

Fig. 6 Caspase-dependent proteolytic cleavage of PKC $\delta$ in vanadium-induced neurotoxicity. (A) Vanadium-induced caspase-mediated PKC $\delta$ cleavage. PKC $\delta$ was immunoblotted after 40 $\mu \mathrm{M}$ vanadium treatment in $\mathrm{N} 27$ dopaminergic neuronal cells with or without the addition of $100 \mu \mathrm{M}$ Z-VAD-FMK for $9 \mathrm{~h}$. Proteins were separated from lysates by $12 \%$ SDS-PAGE and the immunoblot was probed with $\mathrm{PKC} \delta$ antibody to observe both native $(72-74 \mathrm{kDa})$ and cleaved (38-41 kDa) PKC $\delta$ bands. To confirm equal protein loading in each lane, the membranes were reprobed with $\mathrm{B}$-actin antibody. Data represent results from at least two individual measurements. (B) Vanadium-induced PKC $\delta$ cleavage increases the kinase activity. N27 dopaminergic cells were harvested $9 \mathrm{~h}$ after treatment with $40 \mu \mathrm{M}$ vanadium in the presence or absence of $100 \mu \mathrm{M}$ Z-VAD-FMK. Cell lysates were isolated and PKC $\delta$ was immunoprecipitated from treated cell lysates and the enzyme activity was measured by ${ }^{32} \mathrm{P}$ phosphorylation. The values are expressed as a percentage of untreated control cells. Data represent results from at least three individual measurements and are expressed as mean \pm S.E.M. ${ }^{*} \mathrm{p}<0.05$ and ${ }^{* *} \mathrm{p}<0.01$. 
Fig. 7A

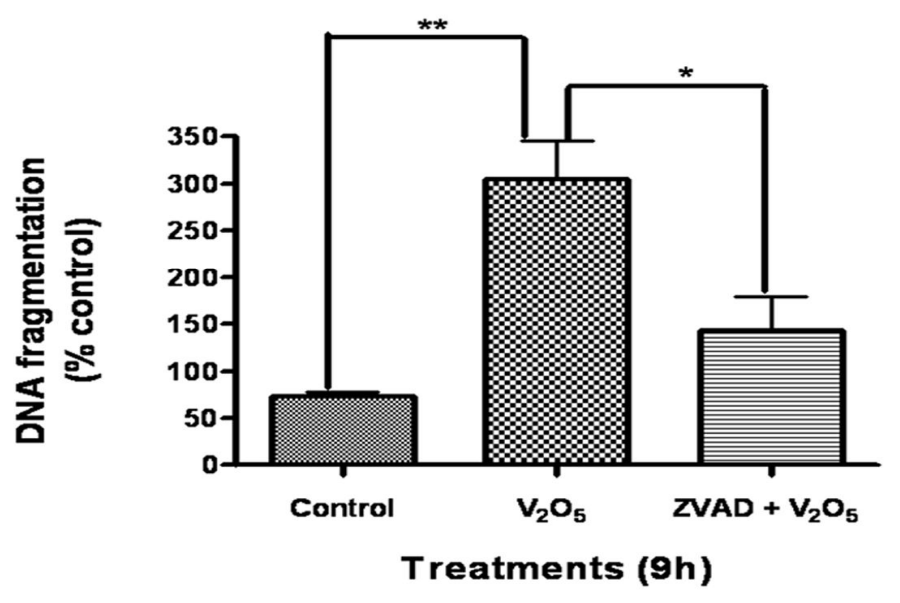

Fig. 7B

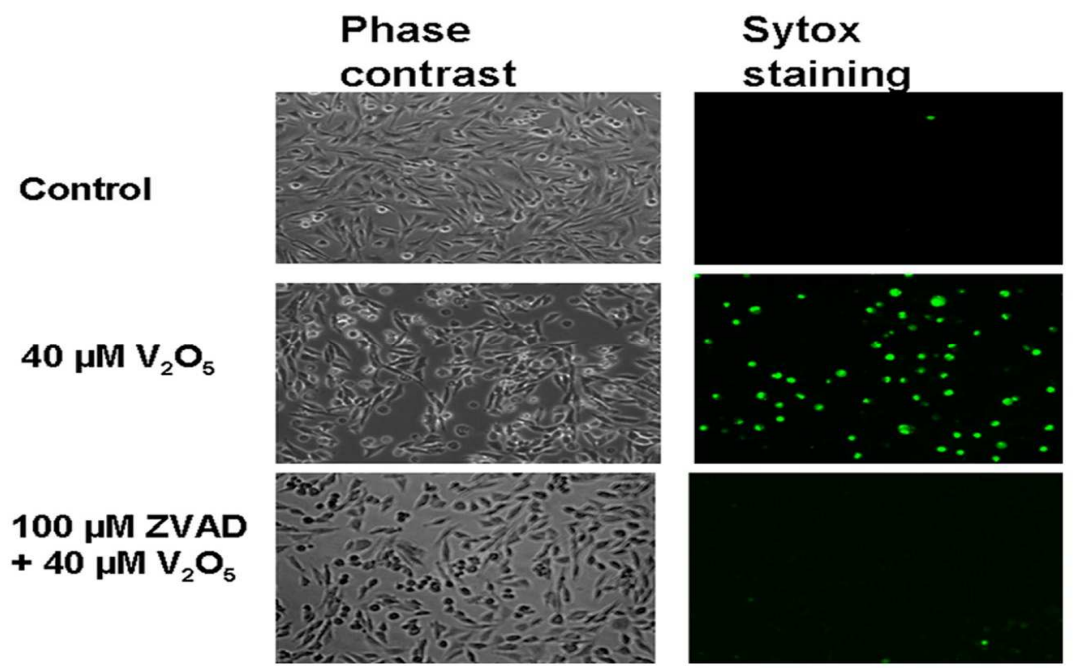

Fig. 7 (A) Effect of Z-VAD-FMK on vanadium-induced DNA fragmentation in N27 dopaminergic neuronal cells. N27 cells were harvested $9 \mathrm{~h}$ after treatment with $40 \mu \mathrm{M}$ vanadium in the presence or absence of $100 \mu \mathrm{M}$ Z-VAD-FMK. DNA fragmentation was quantified using ELISA. The data are expressed as percentage of DNA fragmentation compared to untreated control cells. Data represent results from three individual measurements and are expressed as mean \pm S.E.M. $* p<0.05$ and $* * p<0.01$. (B) Visualization of the protective effect of the pan-caspase inhibitor Z-VAD-FMK on vanadium-induced cytotoxicity. Data represent results from three individual measurements. Sytox green positive cells were observed under a Nikon inverted fluorescence microscope and pictures were captured with a SPOT digital camera (Diagnostic Instruments, Sterling Heights, MI). 
Fig. 8A

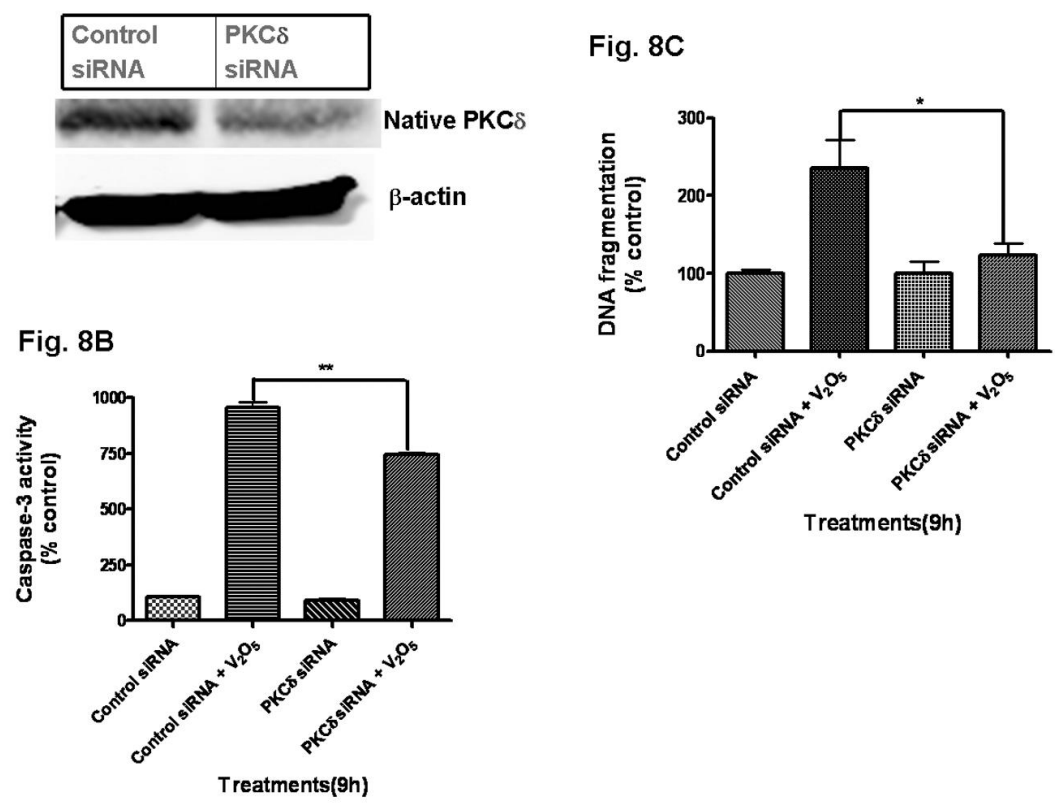

Fig. 8 Effect of PKC $\delta$ knockdown by PKC $\delta$-siRNA on vanadium-induced neurotoxicity. (A) PKC $\delta$-siRNA suppresses $\mathrm{PKC} \delta$ protein expression. N27 cells were transfected with $\mathrm{PKC} \delta$ siRNA ( $25 \mathrm{nM}$ ) or non-specific (NS)-siRNA for $24 \mathrm{~h}$ and PKC $\delta$ expression was determined by Western blotting. Membranes were reprobed with $\beta$-actin $(43 \mathrm{kDa})$ antibody to confirm equal protein loading. Effect of $\mathrm{PKC} \delta$ knockdown on vanadium-induced caspase-3 activity (B) and DNA fragmentation (C). siRNA transfected N27 cells were exposed to $40 \mu \mathrm{M} \mathrm{V} \mathrm{V}_{2} \mathrm{O}_{5}$ for $9 \mathrm{~h}$. Caspase-3 activity and DNA fragmentation were measured as described in the Methods. Data represent results from three individual measurements and are expressed as mean \pm S.E.M. $* p<0.05$ and $* * p<0.01$ 


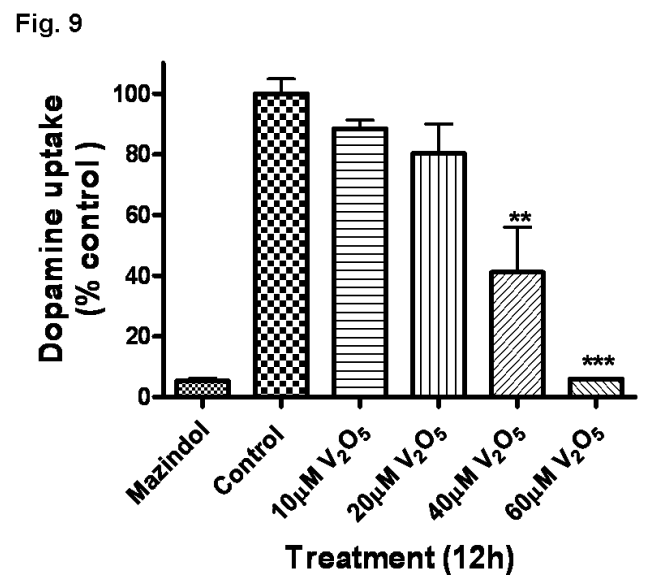

Fig. 9 Neurotoxic effect of vanadium on primary nigral dopaminergic neurons. Mouse primary mesencephalic cultures were exposed to $10,20,40$ and $60 \mu \mathrm{M}$ vanadium for $12 \mathrm{~h}$ and the viability of dopaminergic neurons was measured by ${ }^{3} \mathrm{H}-\mathrm{DA}$ uptake assay. Data represent results from two to six individual measurements and are expressed as mean \pm S.E.M. $* * p<0.01$ and $* * * p<0.001$. 


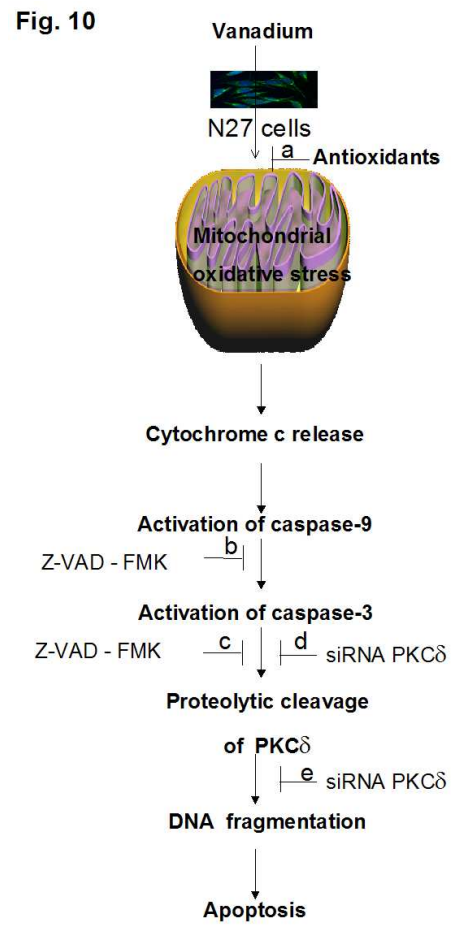

Fig. 10 A model describing the sequence of cell death signaling events in vanadium-induced apoptosis. Vanadium treatment impairs mitochondrial function with an increase in ROS generation, resulting in cytochrome $\mathrm{c}$ release into the cytosol; cytosolic cytochrome c activates the caspase cascade; caspase- 3 mediates proteolytic cleavage of $\mathrm{PKC} \delta$; proteolytically activated PKC $\delta$ mediates DNA fragmentation and apoptosis. Effect of pharmacological inhibitors and genetic modulators on vanadium-induced apoptosis: a, cotreatment with antioxidants prevents cell death; $b$ and c, co-treatment with the pan-caspase inhibitor Z-VAD-FMK prevents proteolytic activation of $\mathrm{PKC} \delta$ and vanadium-induced DNA fragmentation; $d$ and e, RNAi-mediated knockdown of PKC $\delta$ with siRNA- PKC $\delta$ rescues N27 cells from vanadium-induced apoptotic cell death. 